\title{
KINETICS OF DOMAIN FORMATION PROCESSES IN LIPID MEMBRANES
}

Dissertation zur Erlangung des Doktorgrades der

Mathematisch-Naturwissenschaftlichen Fakultäten der Georg-August-Universität

Göttingen

\author{
Vorgelegt von \\ Heiko Seeger \\ aus Eschwege
}

Göttingen 2006 
D7

Referent : Prof. Dr. Tim Salditt

Korreferent : Prof. Dr. Thomas Heimburg

Tag der mündlichen Prüfung : 08 . March 2006 


\section{ACKNOWLEDGMENTS}

Almost ten years ago I entered the world of physics. The motive for studying physics has always been the interest in what I was and am doing. Finally, I have been able to finish the work on my dissertation. That this has been accomplished, however, is not just based on myself, but also on many persons who were involved in it. I would like to thank all of them. They were a great help concerning my research and in my private life.

First of all, I would like to thank my thesis advisor Thomas Heimburg. When I was still a diploma student in his group in Göttingen he offered me to stay in his group. I gladly accepted after a weekend thinking about it, but the question of money needed to be solved. In the end, he put me onto a post-doc position which he transfered to a Ph.D. position. Already this requires thanks, but so also does his support throughout all the years. Each time when the light in the tunnel seemed to diminish he helped to find the next match, which lighted the next candle. I am really grateful for his support.

Tim Salditt showed interest in my work and agreed on being the official supervisor representing the University of Göttingen. This deserves a big handshake and honest thanks.

During my time in the Membrane Biophysics Group in Göttingen and in Copenhagen there were many members whom I would like to send my warm regards. Involved in my work were Agnieszka Hac, Matthias Fidorra, Thomas Schlötzer and Maria Lundgaard Gudmundsson. I would like to thank Agnieszka for the cooperation on the project about lipid diffusion in binary lipid systems. She performed the diffusion experiments which I needed for my Monte Carlo simulations. Matthias was always willing to provide me with beautiful lipid vesicle pictures. Thomas conducted DSC scans so that I could determine the parameters for my simulation. Maria entered our group as a bachelor student. She was so kind to perform experiments which nicely contributed to the success of this thesis. Besides the scientific contributions, I would like to thank all of them for their friendship.

I do not want to forget to thank the other current and former members of the Membrane Biophysics group. In particular these are Peter Grabitz, Martin Gudmand, Vitaliy Oliynyk, Denis Pollakowski and Ivan Makarov. All of them contributed to a friendly and social atmosphere in the group.

I would also like to thank the members of the Biocomplexity group of the Niels Bohr Institute for a very nice time, a kind environment and some exciting table soccer matches.

I am deeply thankful to the workshops in Göttingen and Copenhagen. I always got the help I needed. I am especially thankful to Arno Möller, Eric Kaimar and Bjørn Rasmussen. Arno Möller constructed the new pressure cell and put a lot of effort in it to make it as good as possible. Eric Kaimar and Bjørn Rasmussen spent many hours with me optimizing the construction of the chip calorimeter and giving me helpful advice. Thanks shall also go to Flemming Bo Hansen and Keld Egefelt for their help with any kind of organizational questions in the lab.

As well, I would like to submit my thanks to Inge Dreger, Jan Plotzki and Martin Fechner from the Max-Planck Institute for biophysical Chemistry in Göttingen. They always helped me with administrative, technical or computer problems during my time in Göttingen and continued to do so after I had moved to Copenhagen.

I would like to acknowledge Gert Wolf, Johannes Lerchner and Matthias Jobst from the University of Freiberg for introducing me to use Xensor chips as calorimeters.

I would like to thank Matthias, Mario, Vitaliy, Andrew, Sandeep and Rune for helping me with the correction of this thesis.

I would like to thank all my friends for supporting me throughout all the years. Especially I would like to thank Mario K., Mario F., Olli, Giovanna, Matthias, Vitaliy, Denis, Thomas, Julia, Rune, Brian, Daniel, Jörg, Sebastian, Wolfgang, Martin W. and first of all Federica.

At the end I would like to send my thanks to the ones who followed my path all my life. It is my family. I would like to thank my brother Torsten and his own young family for bringing so much joy into my life. At a time when life seemed to stop, a little being brought so much strength. I would like to thank my brother Jens too. He was patient living with me and he always helped me when I needed something to be done in Göttingen. My parents deserve the biggest thanks of all. They have always supported me in all my decisions. Their unselfishness and support helped to do what I thought was 
the right thing to do and helped me to reach my goal. I will never be able to pay back what they have given to me. Unfortunately, my mother is not able to experience these moments.

This work is dedicated to my parents. 
For my parents ! 



\begin{abstract}
In the vicinity of melting transitions in membranes, fluctuations in enthalpy, volume, area and concentration approach a maximum. Consequences are the formation of domains, increasing elastic constants and permeability, changes in the diffusion behavior and longer relaxation processes.

Molecules such as anesthetics, neurotransmitters and antibiotics play a functional role in biological cells. They also influence the melting behavior of artificial membranes and thereby fluctuation, domain formation, diffusion and relaxation properties.

In this study, the emphasis was on deepening understanding of the domain formation, fluctuation, diffusion and relaxation behavior in the melting regime of artificial membranes. Changes of these processes by anesthetics, neurotransmitters and antibiotics were also investigated. Both experimental and theoretical approaches were used. Fluorescence correlation spectroscopy experiments by A. Hac (Hac, 2004) and pressure perturbation calorimetry were accompanied by statistical thermodynamics simulations. All studied processes are related to each other and are intrinsic properties of the system.

We found that relaxation times slow down in the melting transition regime and the addition of anesthetics, neurotransmitters or antibiotics reduces the maximum relaxation times. Diffusion processes are influenced by the time scale of relaxation processes. Domain formation is accompanied by large fluctuations at domain interfaces.
\end{abstract}

We argue that the studied processes might play a role in the physiology of biological membranes. 


\begin{abstract}
In der Nähe von Schmelzprozessen in Membranen erreichen Fluktuationen der Enthalpie, des Volumens, der Fläche und der Konzentration ein Maximum. Daraus erfolgen Domänenformation, größere elastische Konstanten, eine erhöhte Permeabilität, Veränderungen des Diffusionsverhaltens und längere Relaxationsprozesse.

Moleküle wie Anästhetika, Neurotransmitter und Antibiotika besitzen in biologischen Zellen eine funktionelle Rolle. Sie beinflussen das Schmelzverhalten von künstlichen Membranen und dabei die Fluktuations-, Domänenformations, Diffusions- und Relaxationseigenschaften.

In dieser Arbeit wurde der Schwerpunkt auf das Vertiefen der Kenntnisse über Domänenformationsprozesse, Fluktuationen, Diffusion und Relaxationsverhalten im Schmelzbereich von künstlichen Membranen gelegt. Die Beeinflussung dieser Prozesse durch Anästhetika, Neurotransmitter und Antibiotika wurden ebenfalls untersucht. Sowohl experimentelle, als auch theoretische Methoden wurden genutzt. Fluoreszenzkorrelationsexperimente bei A. Hac (Hac, 2004) und drucksprungkalorimetrische Versuche wurden durch thermodynamische Simulationen begleitet. Alle untersuchten Prozesse hängen miteinander zusammen und sind intrinsische Eigenschaften des Systems.

Wir fanden heraus, dass sich Relaxationsprozesse im Umwandlungsbereich verlangsamen und die Hinzugabe von Anästhetika, Neurotransmitter oder Antibiotika die maximale Relaxationszeit verringert. Diffusionsprozesse werden durch die Zeitskala von Relaxationsprozessen beeinflusst. Domänenformation wird von starken Fluktuationen an den Domänengrenzen begleitet.

Wir argumentieren, dass die untersuchten physikalischen Prozesse eine Rolle in der Physiologie von biologischen Membranen spielen.
\end{abstract}




\section{Contents}

1 Introduction 1

1.1 The Biological Membrane: Its Importance and Its Basic Building Blocks . . . 1

1.1.1 Lipids, Lipid Membranes and Cooperative Melting . . . . . . . . . . . 2

1.1.2 Complex Lipid Membranes and Phase Diagrams . . . . . . . . . . 6

1.1.3 Membrane Models . . . . . . . . . . . . . . . . . . 7

1.2 General Anesthetics, Antibiotics and Neurotransmitters . . . . . . . . . . 10

1.2.1 The Antibiotic Gramicidin A . . . . . . . . . . . . . . . . . . 10

1.2.2 The Neurotransmitter Serotonin . . . . . . . . . . . . . . . . . . . 11

1.2.3 Octanol, Halothane and the Action of General Anesthetics . . . . . . 12

1.3 Diffusion Studies on Membrane Systems . . . . . . . . . . . . . . . . . . 13

1.4 Relaxation Time Studies on Membrane Systems . . . . . . . . . . . . . . . 15

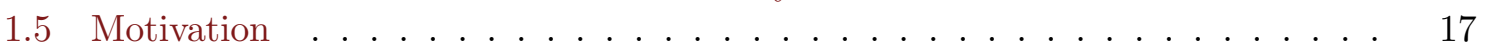

2 Material and Methods $\quad 19$

2.1 Differential Scanning Calorimetry . . . . . . . . . . . . . . . . . . . 19

2.2 Pressure Perturbation Calorimetry . . . . . . . . . . . . . . 20

2.2 .1 Method I . . . . . . . . . . . . . . . . . . . . 21

2.2 .2 Method II . . . . . . . . . . . . . . . . . . 25

2.3 Fluorescence Correlation Spectroscopy . . . . . . . . . . . . . . . . . . 31

2.3 .1 Mathematical Foundations . . . . . . . . . . . . . . 32

2.3.2 Experimental Setup . . . . . . . . . . . . . . . . . . . . 34

2.4 Sample Preparation . . . . . . . . . . . . . . . . . . . . 35

2.5 The Chip Calorimeter . . . . . . . . . . . . . . . . . 37

3 Theory 43

3.1 Modeling Lipid Membrane Systems . . . . . . . . . . . . . . . . . . . . . . . . 43

3.1 .1 Models in the Literature . . . . . . . . . . . . . . . . . 43

3.1.2 A Model for Describing the Phase Behavior of a DMPC:DSPC System 44

3.1.3 Determination of Model Parameters . . . . . . . . . . . . . 46

3.1.4 Evaluation by Means of Monte Carlo Simulations . . . . . . . . . . . . 48

3.1.5 Simulation of Heat Capacity Profiles . . . . . . . . . . . . . . . 50

3.1.6 Time Scales in Enthalpy Traces: Relaxation Time Studies . . . . . . . 51

3.1.7 Simulation of Fluorescence Correlation Spectroscopy Experiments . . 51

3.1 .8 Evaluation of Local Fluctuations . . . . . . . . . . . . . . . 55

3.1.9 Simulation of Temperature Jump Experiments . . . . . . . . . . . . 56

3.2 Relaxation Times in Membranes: An Analytical and Numerical Approach . . 57 
4 Results $\quad 63$

4.1 Experimental and Simulated Phase Diagrams of DMPC:DSPC . . . . . . 63

4.2 Diffusion Studies on Planar Membranes . . . . . . . . . . . . . . . 65

4.3 Melting Transitions of Artificial Membranes . . . . . . . . . . . . . . . 73

4.4 Relaxation Processes of Artificial Membranes . . . . . . . . . . . . . . . 75

4.4.1 Pure DMPC Membrane: Method I . . . . . . . . . . . . . . . . . 75

4.4 .2 Pure DMPC: Method II . . . . . . . . . . . . . . . . . . . . . 79

4.4.3 Addition of Small Molecules or Peptides and Type of Vesicle . . . . . 82

4.4 .4 Temperature Jump Experiments: Simulation . . . . . . . . . . . . . . 90

4.4.5 Relaxation Processes of DMPC:DSPC Mixtures: Simulation . . . . . . 94

4.5 Domain Formation and Fluctuations in Lipid Membranes . . . . . . . . . . . 98

5 Discussion 103

5.1 Discussion of Results . . . . . . . . . . . . . . . . . . . . . 103

5.1.1 Modeling of Melting Processes in a Two Component Lipid System . . 104

5.1 .2 Diffusion in Planar Membranes . . . . . . . . . . . . . . . . . . 105

5.1.3 Melting Profiles of Artificial Membranes . . . . . . . . . . . . . . . . 108

5.1 .4 Relaxation Time Measurements . . . . . . . . . . . . . . . . . . . 110

5.1 .5 Domain Formation and Fluctuations . . . . . . . . . . . . . . 118

5.1 .6 Summary . . . . . . . . . . . . . . . . . . . . 118

5.2 Biological Relevance . . . . . . . . . . . . . . . . . . . . . . 119

5.2.1 Phase Transitions, Lipid Domain Formation and Protein Activity . . 119

5.2 .2 Passive Permeation . . . . . . . . . . . . . . . . . . . . 120

5.2.3 Nerve Pulse Propagation, Ion Channels and Anesthesia . . . . . . . . 121

5.2 .4 Speculations . . . . . . . . . . . . . . . . . . 125

5.3 Future Research Perspectives . . . . . . . . . . . . . . . . . . 126

$5.3 .1 \quad$ Further Experiments . . . . . . . . . . . . . . . . . . . 126

5.3 .2 General Suggestions . . . . . . . . . . . . . . . . . . . 127

A Abbreviations $\quad$ I

$\begin{array}{lll}\text { List of Figures } & \text { II }\end{array}$

List of Tables

$\begin{array}{ll}\text { Bibliography } & \text { V }\end{array}$ 


\section{Chapter 1}

\section{Introduction}

In recent years, research on the physical properties of lipid membranes (aggregates of small amphiphilic molecules) has again resumed popularity, having been a "hot topic" in the 1970s. The interest in lipid membranes arises from its importance in the formation of biological membranes, as it is explained in the first section of this chapter. Without biological membranes, life as it is known nowadays would not have been possible. It is necessary to study the basic physical features of lipid and biological membranes. Different models of biomembranes and a putative role of membrane organization follow from these physical properties. Different classes of molecules influence the physical behavior of lipid membranes and these molecules influence again the function of cells. Examples are anesthetics, neurotransmitter and antibiotics. They are shortly introduced. This thesis is mainly concerned with deepening the understanding of diffusion and relaxation processes of lipid membranes. Therefore, at the end short, reviews about previous studies are given.

\subsection{The Biological Membrane: Its Importance and Its Basic Building Blocks}

Life would not have been possible without the evolution of biological membranes. They allowed a definition of an inner and outer to protect life from a partially hostile environment.

The plasma membrane surrounds biological cells. In cells biomembranes again define organelles of different functionality like the Golgi complex, the mitochondria or the endoplasmic reticulum (see fig. 1.1).

Biological membranes, however, are not just of structural importance but also play a functional role. Permeation processes through the membrane allow communication between the inner and outer part of a cell. They offer the possibility to maintain electrochemical gradients too. Cell membranes are also involved in capturing and releasing energy. Biological membranes reduce the dimensionality of diffusion processes from the three dimensional space to a diffusion in a two dimensional plane. This possibly controls the probability of reactions between two reactants (Adam and Delbrück, 1968).

These functional roles are possible and are influenced by the structure of biomembranes. The main components of biological membranes are small amphiphilic molecules (lipids; 30-80 wt\% of the membrane mass), polymers from amino acids (proteins; $20-60 \mathrm{wt} \%$ of the membrane mass) and carbohydrates (sugars; $0-10 \mathrm{wt} \%$ of the membrane mass). Biological membranes are self-assembled molecular aggregates where lipids play an extraordinarily structural role. 


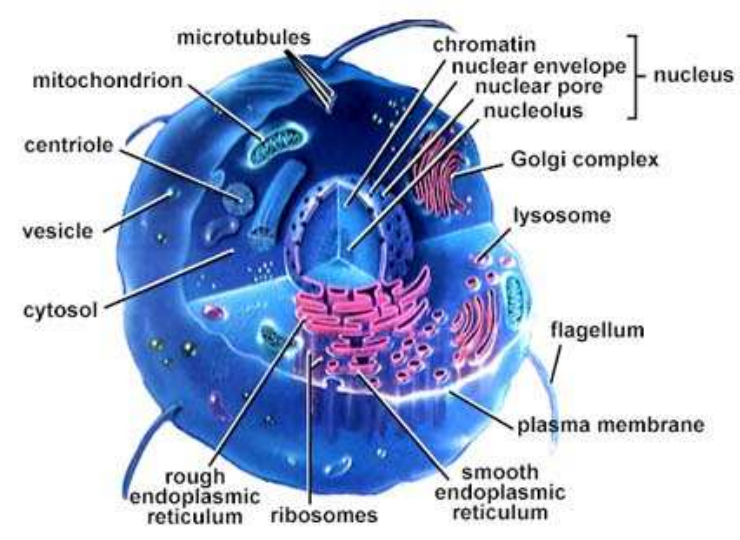

Figure 1.1: Illustration of an animal cell. The plasma membrane defines the inner and outer part of a cell. In the cell itself membranes confine different organelles like the Golgi complex, the endoplasmic reticulum and the mitochondria. These organelles have different functions. Where the mitochondria provide the energy production of a cell, in the endoplasmic reticulum macromolecules of amino acids (proteins) are synthesized. The Golgi complex functions as a central delivery system of proteins of the cell. The picture is taken from "http://www.emc.maricopa.edu/faculty/farabee/BIOBK/BioBookCELL2.html".

\subsubsection{Lipids, Lipid Membranes and Cooperative Melting}

Lipids are small amphiphilic molecules. Many different lipid species ${ }^{1}$ can be found in biological membranes. They can be divided into different families, e.g. sterols or phospholipids. Sterols and phospholipids are the most important lipid species.

\section{1,2-Dimyristoyl-sn-Glycero-3-Phosphatidylcholine}

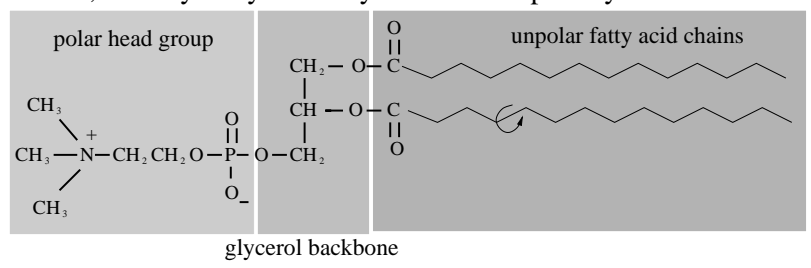

Figure 1.2: Schematic drawing of a DMPC lipid. Each of the two fatty acid chains possesses 14 carbon atoms. The chains are drawn in their all trans configuration. The rotated arrow indicates a possible rotational degree of freedom.

Phospholipids are the most common lipids in biological membranes. They consist of a phosphate containing headgroup and usually two hydrophobic fatty acid chains ${ }^{2}$. Most of the head groups belong to the group of phosphoglycerides which means that the head group and the fatty acid chains are each linked to a hydroxyl of a glycerol backbone (see fig. 1.2). The

${ }^{1}$ It has to be noted that there is no generally accepted definition of the term lipid. This, however, will not be discussed in this thesis. Here, mainly the special class of phospholipids is considered.

${ }^{2}$ Phospholipids with just one unpolar chain can also be found. The majority of phospholipids, however, have two. 
various kinds of phospholipids differ in head group, chain length and degree of saturation of the fatty acid chains.

In fig. 1.2 the chains are drawn in their all-trans configuration. All C-C bonds are in their trans configuration meaning that the potential energy for a rotation about a carbon-carbon bond is in its global minimum (see fig. 1.3). There are two more local minima (gauche confirmations). To perform a rotation to one of the two gauche configurations an energy barrier of about $14.65 \frac{\mathrm{kJ}}{\mathrm{mol}}$ has to be overcome (Gennis, 1989).

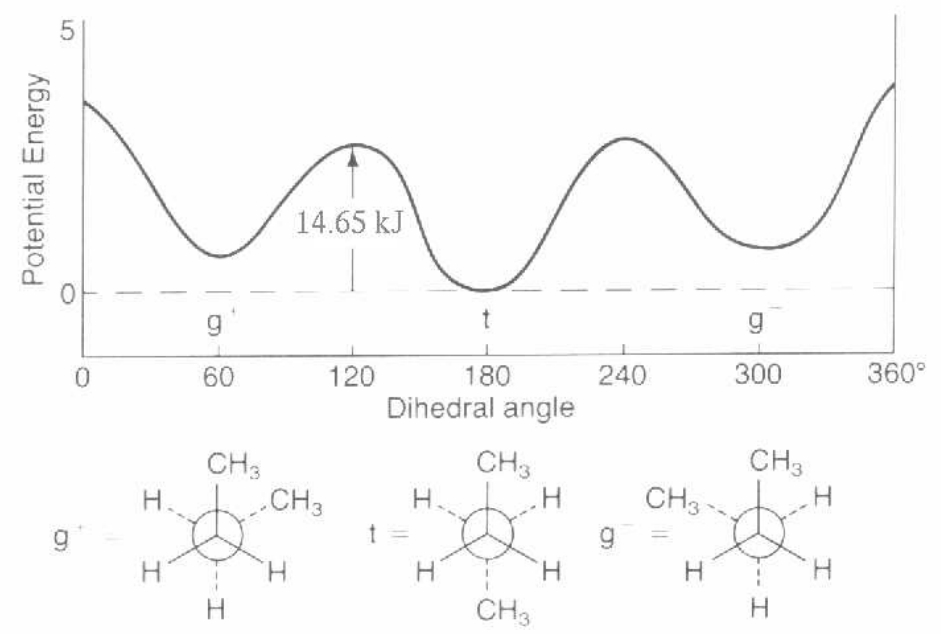

Figure 1.3: Potential energy for rotations of C-C bonds. The potential energy for a rotation of a C-C bond in an alkane is shown in dependence of the dihedral angel. There are three minima. The global minimum is the trans confirmation and the two local minima are the two gauche confirmations $g^{+}$and $g^{-}$. This picture is taken from Gennis (1989).

The probability of forming a gauche conformation depends on temperature. This temperature dependence becomes interesting when talking about lipid aggregates.

Lipids spontaneously form aggregates when given into water, because of their amphiphilic structure. Depending on the concentration and type of lipid, one can observe many different kinds of aggregates (see fig. 1.4). Examples are micelles, inverse micelles and lipid bilayer. The hydrophobic chains are shielded by the head groups from contact with water molecules.

The lipid bilayer structure exhibits a high importance in the formation of biological membranes. At low temperatures the lipids arrange on a hexagonal lattice (Janiak et al., 1979). Lipids are ordered and the degree of rotational freedom of the chains is low. The probability of finding gauche confirmations is small. At high temperatures lipids are disordered with an increased degree of rotational freedom and the lattice structure is lost. In the first case one talks about a solid ordered (so) or gel membrane. In the second case the terms liquid disordered $(l d)$ or fluid ${ }^{3}$ are used. The transition from the "so" to the "ld" phase increases the enthalpy and entropy of the lipid bilayer system. For a one component system this melting transition is highly cooperative, meaning that the melting of one lipid influences the melting

\footnotetext{
${ }^{3}$ The terms gel and fluid are from a physical point of view inexact. Therefore, in this thesis just the terms solid ordered and liquid disordered are used. This terminology was firstly introduced by Ipsen et al. (1987) in the context of the effects of cholesterol on lipid ordering. This is explained further on in the text.
} 

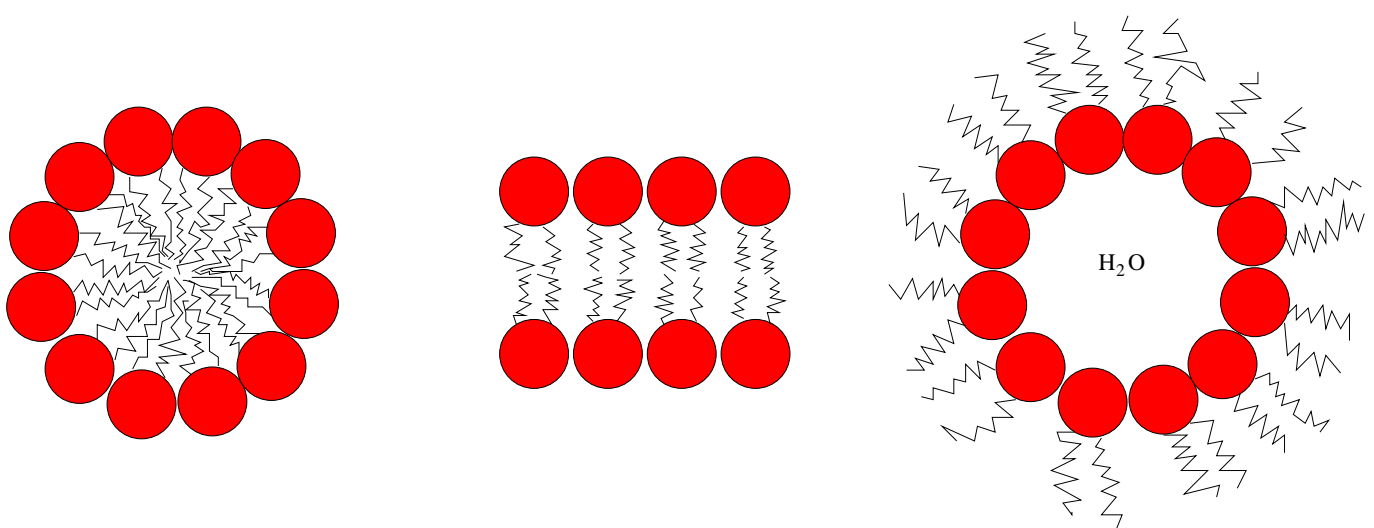

Figure 1.4: Schematic drawing of different lipid aggregates. Shown are representations of (left) micelles, (center) bilayer and (right) inverse micelles.

of another. This is reflected through the small half width of a heat capacity profile. An example is shown in the right panel of fig. 1.5. The point at which the chemical potentials of the two phases equal each other is called the transition midpoint.
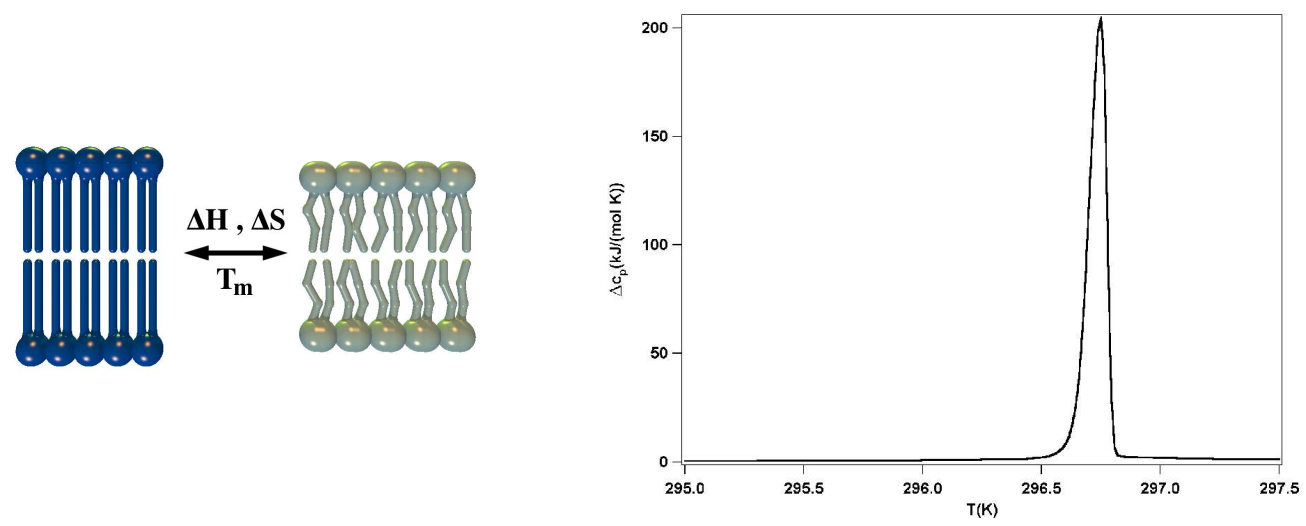

Figure 1.5: Melting transition of a lipid bilayer. (left) The bilayer undergoes a transition from the solid ordered (so) to the liquid disordered $(l d)$ phase. This causes a change in enthalpy $(\Delta H)$ and in entropy $(\Delta S)$. At the transition midpoint $T_{m}$ the chemical potentials of the two phases equal each other. (right) The melting process can be seen in a heat capacity profile. The melting process from the "so" to the "ld" state requires energy which results in an increase in heat capacity.

The transition midpoint, the enthalpy and the entropy all change. The cooperativity of the transition depends on several factors; the kind of head group, the length of the lipid chains, the degree of saturation, the $\mathrm{pH}$, the ionic strength, the outer pressure and the kind of lipid vesicle.

Multilamellar vesicles consist of a stack of bilayers which form vesicles. A suspension of them shows a more cooperative melting than a suspension of unilamellar vesicles. Lipids with different lipid chain lengths also show different melting profiles and they melt at different temperatures (Koynova and Caffrey, 1998). The melting process of the chains is influenced by a balance of competing effects. From an entropic point of view disordered lipid chains are in favor over highly ordered chains in the all-trans configuration. Van-der Waals interactions, however, are increased by the presence of ordered chains. Additionally, close lipid-lipid con- 

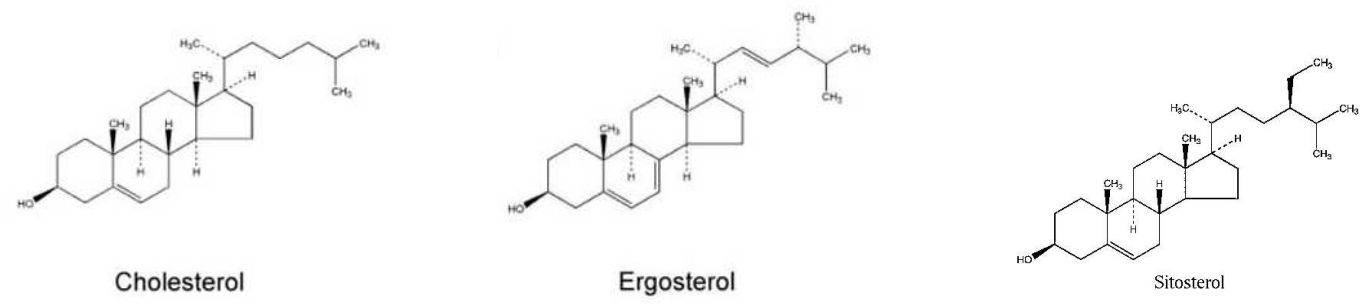

Figure 1.6: Structure of the three sterols cholesterol (left), ergosterol (center) and sitosterol (right). Where cholesterol is mainly found in animal cells, ergosterol is a main part of fungi cells and sitosterol is a phytosterol which is the replacement of cholesterol in plant cells.

tacts can be favored in the case of hydrogen bonds or bridging metal cations. Close contacts, however, may be unfavorable where bulky groups interact sterically or charged lipids electrostatically. Lipids with ordered chains have a smaller cross-sectional area than the ones with disordered chains.

Lipid membranes consisting of lipid species with longer lipid chains melt at higher temperatures than lipid membranes of lipid species with shorter fatty acid chains. The existence of double bonds induces melting at lower temperatures since the ideal interaction of the chains is influenced by the non-existing rotational degree of freedom around the double bond. It is important to note that one component lipid membranes melt in a regime of physiological relevance.

Besides changes in enthalpy and entropy melting also results in an increase of lipid area of about $25 \%$ and an increase of the membrane volume of about $4 \%$. Interestingly physical properties like membrane permeability (Papahadjopoulos et al., 1973; Makarov, 2005) and membrane compressibility and elasticity (Halstenberg et al., 1998; Heimburg, 1998) display maxima in the transition regime. These increases in permeability, compressibility and elasticity are related to area and volume fluctuations.

In the beginning of this chapter it is mentioned that there are two particular families of lipids which have a special status in biological membranes. These are phospholipids and sterols. The first class is introduced in the first part of this section.

Sterols have a different structure in comparison to phospholipids as can be seen in fig. 1.6. Cholesterol is mainly an important component in animal cells. It is replaced by phytosterols in plant cells and by ergosterol in fungi cells.

Physical research has, however, mainly been concerned with cholesterol and its influence on lipid (phospholipid) bilayer. Cholesterol has the tendency to incorporate into the hydrophobic part of the membrane. It influences the phase behavior of a phospholipid membrane, which was discussed for the first time by (Ipsen et al., 1987). Recently, it has been shown that the same effect is true for ergosterol (Beattie et al., 2005). Sterols induce a third phase besides the already described "so" and "ld" phases. This phase is called the liquid ordered (lo) phase. Cholesterol and other sterols order the lipid chain configuration, but at the same time they induce lateral disorder. 


\subsubsection{Complex Lipid Membranes and Phase Diagrams}

As it is described in the previous section one component lipid systems of phospholipids display a phase transition from a "so" phase to a "ld" phase ${ }^{4}$. This picture gets more complicated if cholesterol is added. Besides the "so" and the "ld" phases one finds the lo phase (Ipsen et al., 1987). A coexistence of phases is possible which depends on temperature and cholesterol concentration. In the case of more complex lipid systems with two different phospholipid species structural heterogeneities occur over a broader temperature regime. This can be seen in a heat capacity profile as it is shown in fig. 1.7.
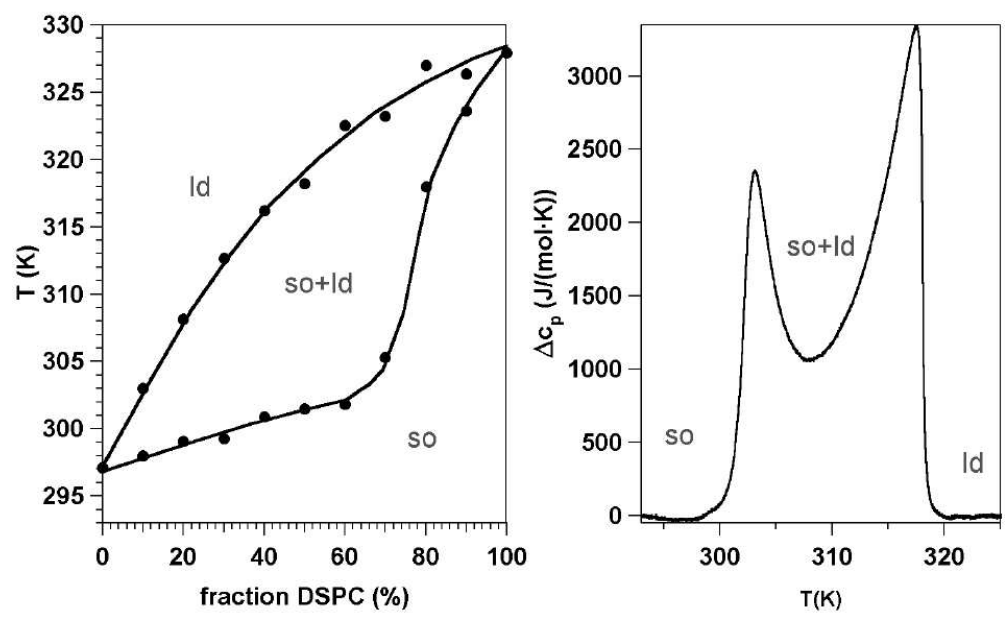

Figure 1.7: Phase diagram and excess heat capacity profile of a two component lipid system. (right) An example of a measured $\Delta c_{p}$-profile of a two component lipid system is shown. From a series of heat capacity profiles of the same lipids with different molar ratios one can construct a phase diagram (left). Out of this phase diagram one can deduce at which ratios and temperatures the system is in one of the pure phases (either "so" or "ld") or when it is in a coexistence regime.

A heat capacity anomalie is monitored over a broad temperature regime. At low temperatures and at high temperatures the lipid membrane is in either the "so" or the "ld" phase. In the region of non-zero $\Delta c_{p}$, coexistence of "so"-like and "ld"-like domains occur. These properties are equivalent to the ones of "so" and "ld" phases. Dependent of ratios, concentrations or temperature, phase diagrams give information whether one is in a coexistence regime or one of the pure phases (see the left panel of fig. 1.7). They allow one to approximate the ratio of ordered to disordered lipids in the coexistence regime (Lee, 1977), but they do not give any information about the morphology of domains. They can be constructed with the help of excess heat capacity profiles. The details of the construction is disputed (Sugar et al., 2001).

Recently, phase separation of two component lipid bilayer systems of Giant Unilamellar Vesicles (vesicles of diameter $10 \mu m-500 \mu m$; GUV) have been visualized by Confocal Fluorescence Microscopy (Korlach et al., 1999; Bagatolli and Gratton, 1999). Fluorescent labels

\footnotetext{
${ }^{4}$ It is neglected that for MLVs one measures a pre-transition. The lipid membrane undergoes a transition from the "so" to the ripple phase. The ripple phase is due to line defects of disordered lipids (Heimburg, 2000). The enthalpy change is much smaller than the one for the main phase melting transition. For reasons of simplicity this transition stays neglected in most parts of this thesis.
} 
which partition differently into the two kinds of domains or whose fluorescence light wavelength depends on the state of the lipid environment are incorporated into the lipid bilayer. After excitation through a laser the fluorescence is monitored. An example is shown in fig. 1.8 (provided by M. Fidorra; (Fidorra, 2004)).

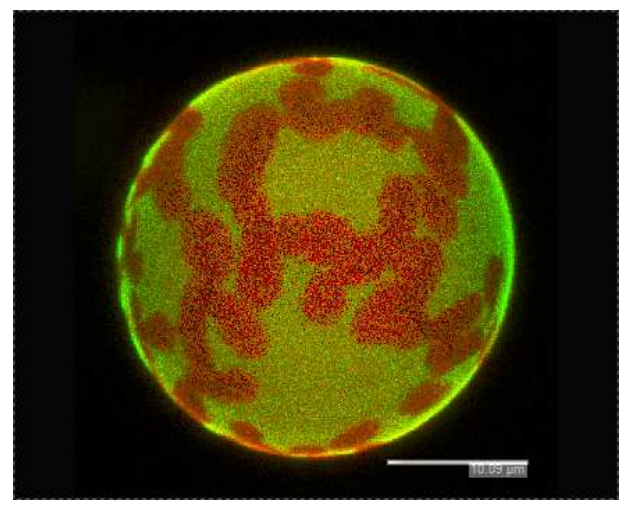

Figure 1.8: Confocal Fluorescence Microscopy picture provided by M. Fidorra (Fidorra, 2004). Two fluorescent markers are included into the phospholipid bilayer (DLPC:DPPC 67:33) of a Giant Unilamellar Vesicle (GUV; vesicles of diameter $10 \mu m-500 \mu m$ ). One of them preferentially partitions into "so" domains whereas the other one prefers a "ld" environment. After excitation the two markers emit light of different wavelengths which allows one to monitor the different domains. The red and green colors represent "so" and "ld" domains. The scale bar is $10 \mu m$.

The size of these domains is debated in the literature. Where fluorescence pictures show domains in the range of a few $\mu m$ (Korlach et al., 1999; Bagatolli and Gratton, 1999; Seeger et al., 2005), Atomic Force Microscopy (AFM) ${ }^{5}$ of supported lipid bilayer ${ }^{6}$ systems suggest domains in the $\mathrm{nm}$ regime (Nielsen et al., 2000b; Kaasgaard et al., 2002; Tokumasu et al., 2003).

\subsubsection{Membrane Models}

In the previous two sections it is discussed that lipid systems consisting of one or two different kinds of phospholipids, with or without cholesterol, display a complex phase behavior. Depending on temperature and lipid ratios, heterogeneities in the lateral membrane structure can be found. This can already be deduced from heat capacity profiles, but is visualizable by Confocal Fluorescence Microscopy or Atomic Force Microscopy. The first measurements of heat capacity anomalies of lipid membrane systems and also biological membranes date back to the late 1960s and the 1970s (Steim et al., 1969; Reinert and Steim, 1970; Hinz and Sturtevant, 1972; Melchior and Steim, 1976; Mabrey and Sturtevant, 1976; Jackson and Sturtevant, 1977). This would suggest that a picture describing a biological membrane is a rather heterogenous than homogenous one.

However, the accepted text book picture of a biological membrane is still the fluid mosaic model (Singer and Nicolson, 1972). The fluid mosaic model is based on a fluid ("ld") lipid

\footnotetext{
${ }^{5}$ Atomic Force Microscopy allows one to gain a hight profile of a surface. Applying it to the study of lipid membranes it detects hight differences of the different lipid membrane phases.

${ }^{6}$ A supported lipid bilayer is a bilayer which is brought onto a surface and which has close contact to this one.
} 
membrane which builds a matrix for randomly distributed proteins. Proteins might incorporate into the membrane (integral proteins) or are attached onto the membrane surface (peripheral proteins).

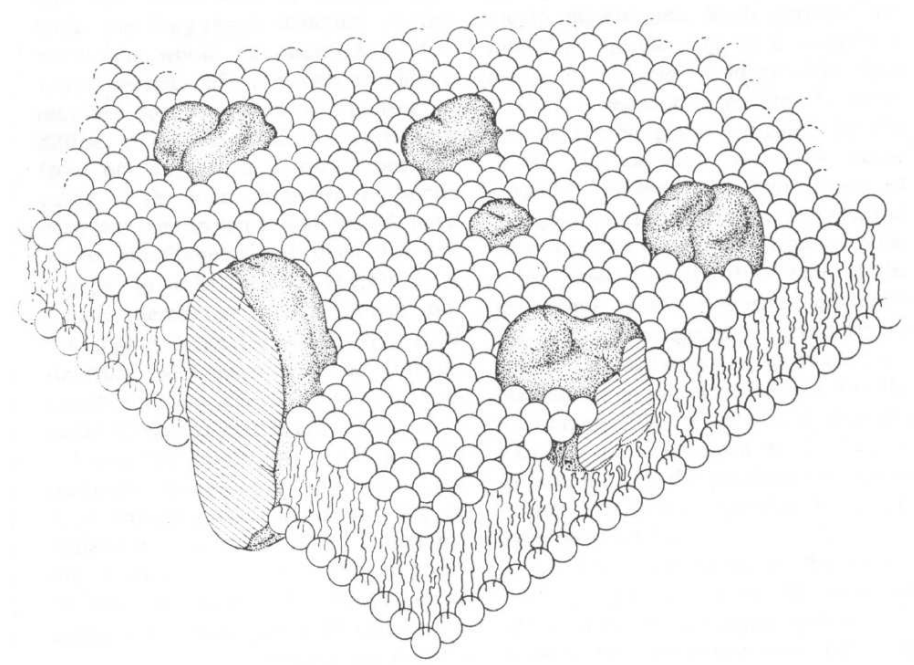

Figure 1.9: Fluid mosaic model of cell membranes. The lipids (small objects with tails) are in a disordered state and provide a fluid matrix for the randomly distributed proteins (rigid bodies). The picture is the same as fig. 3 in Singer and Nicolson (1972).

This model suggests a rather homogenous picture of a biological membrane. Singer and Nicolson (1972), however, it expressed the idea of possible heterogeneities in the vicinity of proteins. In addition, the idea that physical or chemical perturbations of the membrane might affect or alter membrane components has been mentioned. In summary, however, the lipid components do not receive much attention besides providing a viscous medium for the membrane proteins.

For the first time a membrane model implementing clusters of lipids was formulated by Jain and White (1977): "... the biomembrane continuum is broken up into a number of relatively rigid plates or patches that are in relative motion with respect to each other.". Though, this model did not gain much attention. After the proposition of the existence of specialized domains, so called "rafts" (Simons and Ikonen, 1997), this idea is under discussion. "Rafts" are seen as being lo lipid domains which are rather static in structure. They float in a "ld" environment. From a thermodynamical point of view this is rather unlikely since "rafts" are seen as being small clusters (Simons and Vaz, 2004). Small lipid domains are likely to show strong local fluctuations in lipid chain state and therefore, they are more likely dynamic than static (Seeger et al., 2005).

The insufficiency of the fluid mosaic model also becomes clear from diffusion studies on biological membranes. A rather complex diffusion behavior is revealed which cannot be explained by a homogenous membrane system (Jacobson et al., 1995). One possible reason for the occurrence of inhomogeneities is the existence of lipid domains, which are governed by

\footnotetext{
7 In the literature the definition of the term raft is rather confusing. Most authors talk about it in the context of the formation of domains with a high content of cholesterol and sphingolipids (phospholipids where the glycerol backbone is replaced by a sphingosine).
} 


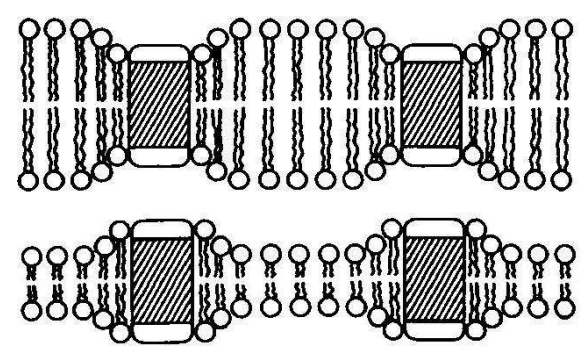

Figure 1.10: Matress model of lipid-protein interactions. It has been proposed by Mouritsen and Bloom (1984). The hydrophobic mismatch between the proteins and the lipids result in a deformation of the lipid matrix so that the hydrophobic mismatch is kept as small as possible (fig. 1 from (Mouritsen and Bloom, 1984)).

lipid-lipid and lipid-protein interactions. The latter ones have been included in the mattress model by Mouritsen and Bloom (1984). The lipid membrane is deformed so that the hydrophobic mismatch between proteins and the lipid membrane is minimized (see fig. 1.10). The principal of hydrophobic matching is seen as an important factor in influencing protein function (Dumas et al., 1999; Jensen and Mouritsen, 2004).

Another putative reason for the occurrence of heterogeneities is aggregation of proteins or peptides. Light-induced aggregation behavior of bacteriorhodopsin, a proton transporting membrane protein, has been measured through a change in diffusion behavior (Kahya et al., 2002). Aggregation behavior has also been found in numerical simulations for different proteins and peptides (Heimburg and Biltonen, 1996; Ivanova et al., 2003). These simulations are based on comparison to measured heat capacity profiles. They also provide information about the lateral membrane structure. Proteins might favor one of the lipid membrane phases.

All in all one can conclude that the simple picture as it is drawn in the fluid mosaic model has to be refined. If one thinks about a biomembrane one should rather think of a heterogenous than of a homogenous lateral structure. A visualization of this picture is shown in fig. 1.11. The membrane lipids can be in different states and might form domains with different physical behavior ("so", lo and "ld"). Proteins might have a tendency towards one of the particular lipid domains and might aggregate to clusters themselves. It is known that the lipid composition of the two monolayer of the same membrane may vary (Bretscher, 1972; Devaux, 1991). Whether the state of the lipid might differ in both leaflets is not answered, yet.

Despite the representation in fig. 1.11 the topology of a biological membrane is more complex and includes curvatures due to different domain heights and hydrophobic matching.

This modern view of a biological membrane allows the triggering of biochemical reaction cascades due to the physics of the lipid membrane. Diffusion properties of proteins change. Protein function might also be influenced by the lipid environment and the state of the lipids. The lipid itself gets a more active role concerning the physiology of a cell than is the case in the fluid mosaic model. This possible role is discussed in more detail in a later chapter (see ch. 5). 


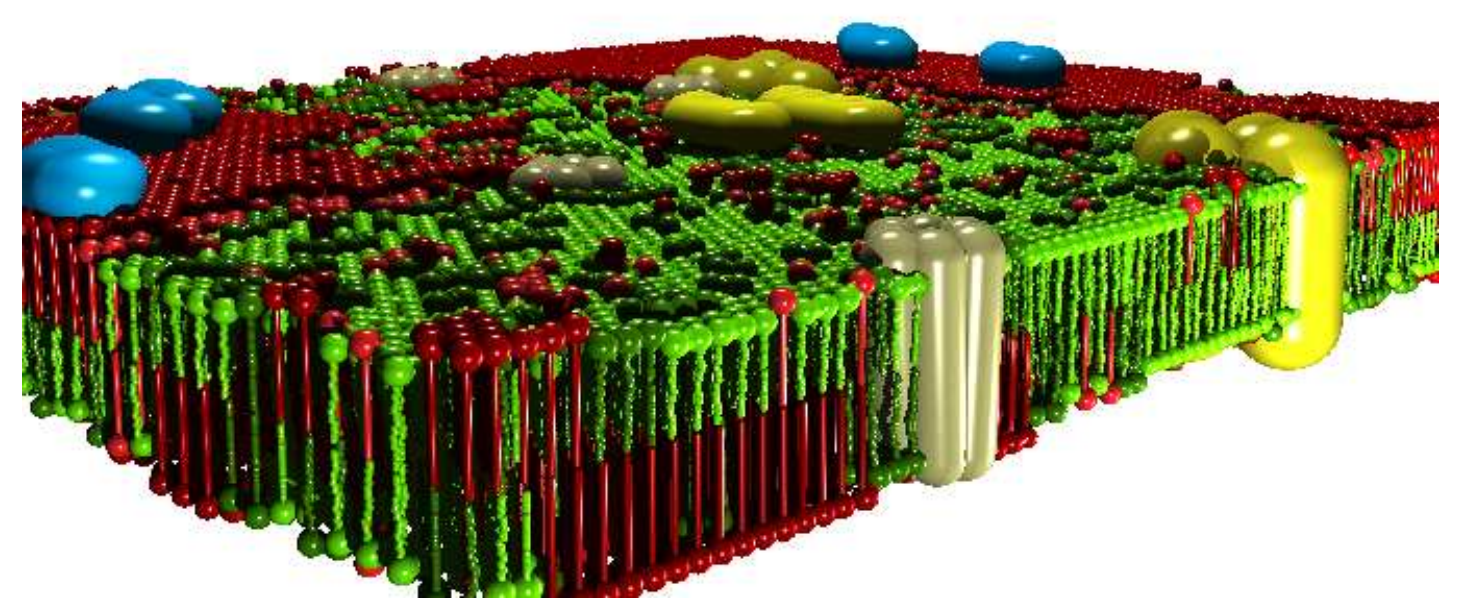

Figure 1.11: A modern picture of a biological cell membrane. The different colors of the smaller objects represent lipids with an ordered (red) or disordered (green) configuration. Lipid domains with different physical properties are formed. The aggregation of proteins (rigid, big objects) is allowed. Proteins might prefer ordered or disordered lipids. The two monolayers of the bilayer differ in lipid composition and the lateral domain structure may be dissimilar, too. This model allows the control of biological function because of the physics of the lipid membrane.

\subsection{General Anesthetics, Antibiotics and Neurotransmitters}

The most important components in biological membranes are lipids, proteins and sugars. In this thesis sugars and their importance and influence on membrane physiology are not considered. Thus, they are not discussed further.

Proteins are assumed to be the functional units of biological membranes. The physical properties and the structure of a biomembrane might influence the function of a protein. Different proteins and peptides accomplish different tasks. The peptide gramicidin A acts as an antibiotic, whereas other proteins, so called channel proteins, are thought to play a role in nerve pulse propagation.

The function of a biological membrane, however, can also be influenced by small molecules like octanol or halothane. These two molecules belong to the class of general anesthetics. Neurotransmitters like serotonin have an influence on e.g. the mood of human beings.

In this thesis, studies on phospholipid membranes with gramicidin A, the two anesthetics halothane and octanol and with serotonin are presented. Therefore, the physical and chemical properties of these molecules and the current hypothesized action of these molecules is introduced in the following three sections.

\subsubsection{The Antibiotic Gramicidin A}

The hydrophobic peptide gramicidin A (see fig. 1.12) is thought to be a channel forming peptide specific for monovalent cations. The channel is a dimer of the peptide (Gennis (1989) and citations therein). Its size is $30 \AA$ in length and the inner and outer diameters are $5 \AA$ 
and $15 \AA$, respectively.

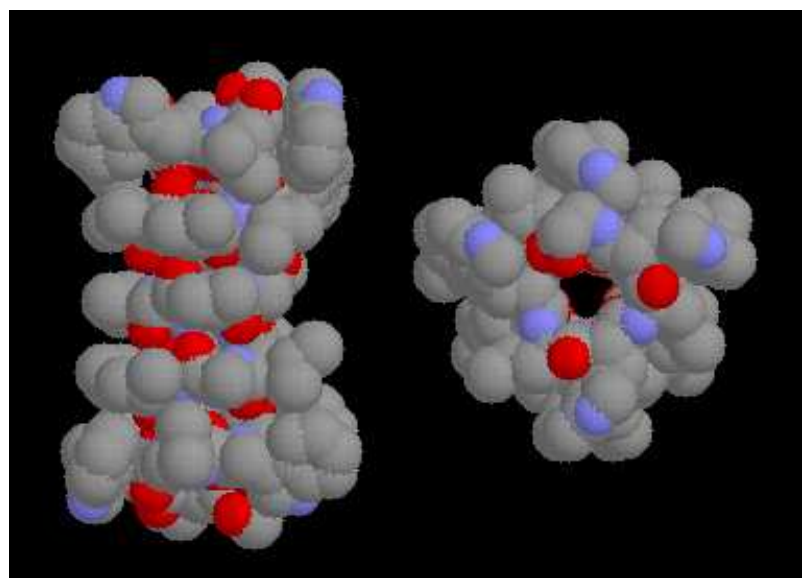

Figure 1.12: The peptide gramicidin A. It is a channel forming peptide which acts as an antibiotic. The picture is generated from a protein data bank file of the Research Collaboratory for Structural Bioinformatics of the Brookhaven National Laboratory (www.rcsb.org; see file 1Mag.pdb).

Gramicidin A acts as an antibiotic. This is believed to be due to its channel forming behavior. Cations can freely diffuse through the channel and ion gradients get destroyed. This leads to the death of the cell.

\subsubsection{The Neurotransmitter Serotonin}

Neurotransmitters are molecules which mainly occur in the nerve system, but can also be found in other parts of a body. They can be classified into two groups: small molecule transmitters and neuroactive peptides. It is assumed that they play an important role in nerve pulse propagation. During a nerve pulse they are released into the synaptic gap between a pre- and postsynaptic neuron ${ }^{8}$. The released neurotransmitters bind to receptors of the postsynaptic neuron. It is believed that the effect of the neurotransmitter is dictated by the receptor. One class of receptors are protein channels which go into an open or closed state after the binding of a neurotransmitter. This allows triggering of the membrane potential. A second class of receptors is coupled to intracellular second messenger cascades which, however, also influence protein channels and therein again the membrane potential. Neurotransmitters can be excitory or inhibitory, meaning that they can induce or prevent a nerve impulse.

Serotonin (5-Hydroxytryptamin; see fig. 1.13) is a small molecule transmitter which has two aromatic rings. It is believed to play an important role in depression, migraine and anxiety. Serotonin acts as an excitory neurotransmitter which is extensively found in the human blood stream. It is also present in the human gastrointestinal tract. $1 \%-2 \%$ of the Serotonin concentration in the human body is localized in the brain region.

${ }^{8}$ Synapses are junctions between nerve cells (neurons). Nerve pulses are accompanied by directed action potentials. The neuron which seen from the direction of the action potential is before the synaptic cleft is a presynaptic one. The one after the gap is the postsynaptic neuron. 


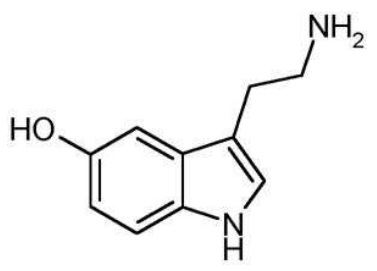

Figure 1.13: The neurotransmitter serotonin. It consists of two aromatic rings and is an excitory neurotransmitter. serotonin is involved in systems of human brain regions which are responsible for mood, emotional behavior and sleep.

\subsubsection{Octanol, Halothane and the Action of General Anesthetics}

In the mid 19th century it was found that certain gases allow the blocking of pain, consciousness and other sensations. This state is called anesthesia and since then has gained importance in medicine. Nowadays, one divides anesthetics into general, local or regional anesthetics. The latter one means a reversible loss of sensation and possibly movement in a certain region of the body through blocking brain or nerve sections which are responsible for this region. Local anesthetics act reversible in a defined part of the body through direct injection of anesthetics. The first one, general anesthetics, induce a reversible loss of conscious and sensation. Here, the focus is on general anesthesia.
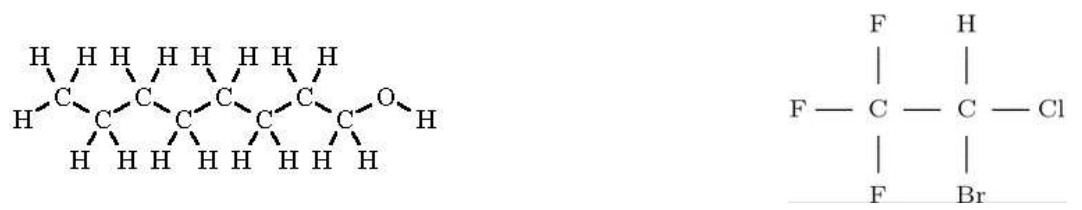

Figure 1.14: The general anesthetics octanol (left) and halothane (right). The alcohol 1-octanol and the halogenated hydrocarbon halothane act as general anesthetics. There is no generally accepted theory of general anesthesia, yet.

Different kinds of small molecules induce general anesthesia. One has chosen a somehow arbitrarily and rough division of anesthetics into three classes: volatile and anesthetics gasses, alcohols and intravenous anesthetics. Halothane (2-Bromo-2-chloro-1,1,1trifuoroethane; $\mathrm{BrCH}(\mathrm{Cl}) \mathrm{CF}_{3}$; see fig. 1.14), a halogenated hydrocarbon belongs to the first class of anesthetics. Alcohols up to a chain length of ten carbon atoms act also as anesthetic drugs. An example is octanol $\left(\mathrm{C}_{8} \mathrm{H}_{18} O\right.$; see fig. 1.14) which has eight carbon atoms. To the last group of intravenous anesthetics belong barbiturates like pentobarbitone.

The pioneering works in the field of anesthetics were independently published by Meyer (1899) and Overton (1901). They showed that the anesthetic potency of a substance depends on their solubility in olive oil (Meyer-Overton rule). As well, they showed that the effect of anesthetics add up. A whole variety of different substances could induce anesthesia and it could be concluded that those anesthetics used acted nonspecifically. The effect of anesthetics can be reversed by applying pressure or by heating (Spyropoulos, 1957).

Already Overton (1901) suggested that anesthesia is a result of the perturbation of the lipid membrane. The first theories of anesthesia were dominated by explaining the action of anesthetics on the lipid membrane and an indirect lipid-mediated mechanism (Lee, 1976b; 
Trudell, 1977). Three main objections have been brought up against lipid-mediated theories. These are the failure of the Meyer-Overton rule of explaining the lack of anesthetic activity of long-chain alkanes and alcohols, the small magnitude of effects of anesthetics on the lipid membrane at clinical relevant concentrations and the third argument is the stereoselectivity $^{9}$ of some substances. This has led to the dominance of theories explaining anesthetics action because of the influence of anesthetics on protein function (Franks and Lieb, 1994; Krasowski and Harrison, 1999; Bovill, 2000).

Still others argue in favor of the influencing effect of the anesthetics on the lipid membrane. Ueda and Yoshida (1999) claims that it is a combination of effects on proteins and lipids. The actual side of action is the lipid/water interface. Cantor (Cantor, 1997, 1999b, 2001b) believes that the lateral pressure profile of lipid bilayer controls the function of channel proteins. He discusses the action of anesthetic molecules in the context of their influence on the lateral pressure profile.

In a recent paper, Heimburg and Jackson (2005) challenge the traditional picture of nerve pulse propagation. They claim that the nerve pulse is a soliton which is the natural result of a jump through or into the transition regime of a biological membrane. Taking this theory they explain the action of general anesthetics as being due to the influence of anesthetics on the transition profile of the lipid membrane (Heimburg and Jackson, 2006). Short chain alcohols shift the profile to lower temperatures and therewith increase the threshold of the evolution of a soliton.

In total one can conclude that despite the fact that the effect of general anesthesia has been known for more than 150 years, still the precise reason of anesthesia is still not fully understood.

\subsection{Diffusion Studies on Membrane Systems}

In previous sections it is already mentioned that a modern picture of a biological membrane includes heterogeneities. Nowadays, it is more and more accepted that these homogeneities play a crucial role in membrane function. They influence diffusion properties which are believed to be important in trafficking processes.

The diffusion behavior in a biological membrane is rather complex (Jacobson et al., 1995). Diffusion in biomembranes often deviates from normal diffusion, where the mean-square displacement $(m s d)$ is proportional to time (Einstein $(1905,1906)$; $m s d=4 D t$, with $D$ the diffusion constant). Diffusion in biological membranes can sometimes be described by an anomalous diffusion behavior. The proportionality of the $m s d$ with time $t$ is replaced by $t^{\alpha}$, where $0<\alpha \leq 1$. Formally speaking, this means that the diffusion constant is not a constant any more but a variable depending on time. Diffusion in confined areas, from which the diffusing particle cannot escape, can be monitored too. Due to active processes diffusion might be directed.

In order to gain a deeper understanding of the underlying physical processes experimental and theoretical studies have been applied to artificial and biological membrane systems. A range of different experimental techniques have been chosen. The most common ones

${ }^{9}$ The enantiomers of etomidate, which is an intravenous anesthetic, were shown to have a 15 -fold difference in the anesthetic potency on tadpoles. They show, however, identical effects on the physical properties of the lipid bilayer (Tomlin et al., 1998). 
are fluorescence recovery after photobleaching ${ }^{10}$ (FRAP; (Vaz et al., 1982; Schneider et al., 1983; Vaz et al., 1982, 1989; Almeida et al., 1992; Feder et al., 1996)), fluorescence correlation spectrscopy ${ }^{11}$ (FCS; (Eigen and Rigler, 1994; Korlach et al., 1999; Schwille et al., 1999; Wachsmuth et al., 2000; Böckmann et al., 2003)), single particle tracking ${ }^{12}$ (SPT; (Simson et al., 1995; Schütz et al., 1997; Sonnleitner et al., 1999; Ritchie et al., 2005)), nuclear magnetic resonance ${ }^{13}$ (NMR; (Kuo and Wade, 1979; Crawford et al., 1980; Orädd et al., 2002; Arnold et al., 2004; Scheidt et al., 2005)), electron spin resonance ${ }^{14}$ (ESR; (Shin et al., 1991)), fluorescence quenching ${ }^{15}$ (Galla et al., 1979) or quasielastic neutron scattering ${ }^{16}$ (QENS; (Pfeiffer et al., 1988; Tabony and Perly, 1990)).

The various methods measure diffusion on different length scales. FRAP and FCS map diffusion on long length scales, whereas fluorescence quenching and QENS monitor it on short length scales. SPT, NMR and ESR can be used for studying diffusion on long and short length scales, depending on the details of the experiment. Measuring of short and longe range diffusion in one experiment is possible too (Shin et al., 1991).

Measurements mapping diffusion on different length scales show differences in the diffusion constants. Discrepancies are explained by different diffusion processes. Microscopic (short length scales) and macroscopic (long length scales) diffusion is distinguished in the literature (Vaz and Almeida, 1991; Shin et al., 1991). Almeida and Vaz (1995) argue that methods which measure lipid movements over the order of lipid size are not likely to be related to the diffusion coefficient since the latter one is an average property. The macroscopical diffusion coefficient is smaller than the microscopical one.

Not considering the discrepancy between microscopic and macroscopic diffusion constants one also finds deviations of reported diffusion constants which measure diffusion over long length scales. This is especially the case for diffusion in the "so" phase. Authors using FRAP report diffusion in "so" membranes which is much smaller (several orders of magnitude) than the ones gained with FCS.

For a complete understanding of diffusion processes in biological and artificial membranes theoretical approaches need to be found. For the description of protein diffusion in a homogenous environment hydrodynamic theories succeeded (Saffman and Delbrück, 1975; Hughes et al., 1982). The protein is considered as a cylinder which diffuses in a viscous medium, the lipid membrane. This theory, however, fails in the description of the diffusion of lipids since the lipids are of the size of the particles of the viscous medium. A hydrodynami-

${ }^{10}$ Fluorescence labels in a defined area in the system under investigation become bleached by a high intensity of laser light. After that diffusion of other labels results in a recovery of the detected fluorescence signal.

${ }^{11}$ Concentration fluctuations of fluorescence labels in a confocal volume result in fluctuations of the monitored fluorescence signal. In diffusion studies these fluctuations depend on the diffusion properties.

12 The track of a single particle is monitored. This allows the determination of the $m s d$.

${ }^{13}$ In nuclear magnetic resonance measurements one excites the spins of the atom's nuclei. There is a variety of NMR techniques which allow the study of diffusion processes. The NMR echo contains information of the diffusion processes.

${ }^{14}$ Electron spins of labels are excited and the spectral intensity is monitored. Dynamic imaging allows the analysis of the data by means of diffusion processes.

${ }^{15}$ A fluorescence molecule in the ground-state and one in an excited state might form an excimer (two molecules bound in an electronic excited state). A diffusion constant can be defined which depends on the ratio of the fluorescence intensity of the excimer and the monomer.

${ }^{16}$ A monochromatic neutron beam is scattered. Due to molecular motion, energy is transfered between the neutrons and the sample and results in a broadening of the energy profile. The broadening depends on the time scale of the molecular motion. The broadening depends also on the angle of the neutron beam which again yields information about the type and spatial extend of the motion. 
cal theory requires that the size of the particle under consideration is much bigger than the particles of the viscous medium. In the case of lipid diffusion, phenomenological "free volume theories" have been successfully applied (Galla et al., 1979). In these theories the diffusion of a particle is possible if there is enough "free volume" which the particle can occupy.

However, both the hydrodynamical theories describing protein diffusion and the "free volume theories" fail to describe the diffusion processes in heterogenous systems. Therefore, especially M. Saxton (Saxton, 1987, 1990, 1992, 1994, 1996) has used numerical methods to model and understand possible hindrances to diffusion in biological membranes. Saxton applies hardcore models and evaluates them by means of Monte-Carlo simulations. He describes different reasons for an influence of diffusion processes. These are the existence of mobile obstacles (Saxton, 1987), aggregation behavior (Saxton, 1992) or binding processes (Saxton, 1996). He analyzes some of the results by means of anomalous diffusion (Saxton, 1994, 1996).

One problem of these hard-core models, however, is that they do not contain any thermodynamical information. In more recent works (Polson et al., 2001; Hac et al., 2005; Sugar and Biltonen, 2005) simple lattice models, that can describe the thermodynamics of lipid/sterol or two component lipid systems, are used for the study of diffusion processes. They allow the examination of diffusion in artificial membrane systems and its dependence on temperature and composition. The results are discussed elsewhere (see ch. 4 and 5).

The importance of diffusion processes in biological functions was pointed out in the mid 1980s (McCloskey and Poo, 1984; Axelrod, 1983). After the start of the discussion about "rafts" and its implications in trafficking processes it has become even more evident. As it has been explained earlier, heterogeneities in biomembranes, e.g. because of lipid domains, influence the diffusion behavior and therefore, possibly allow the triggering of biochemical reaction cascades.

\subsection{Relaxation Time Studies on Membrane Systems}

The putative role of phase transitions in biological membranes was already discussed in the beginning of the 1970s (Chapman, 1971; Träuble, 1971). After the publication of the fluid mosaic model (Singer and Nicolson, 1972) evidence of the importance of the mechanical and thermodynamical properties of lipid membranes were gathered (van Osdol et al., 1989). This included studies of statical properties of phase transitions, but also kinetic measurements of the phase transition process.

The first kinetic experiments were conducted in the early and mid 1970s (Träuble, 1971; Tsong, 1974; Tsong and Kanehisa, 1977). They were based on a sudden jump in temperature. In the following years studies with a variety of different techniques and detection methods followed. Perturbation techniques like temperature perturbation (Holzwarth and Rys, 1984; Holzwarth, 1989; Jørgensen et al., 2000; de Almeida et al., 2002), pressure perturbation (Clegg et al., 1975; Gruenewald et al., 1980; Elamrani and Blume, 1983; Yager and Peticolas, 1982; Cheng et al., 1994; Grabitz et al., 2002), volume perturbation (van Osdol et al., 1989) or changing $\mathrm{pH}$ or ion concentration (Strehlow and Jähnig, 1981) were used. Different nonand thermodynamical methods were used for the detection of the kinetic behavior. Optical detection modes like turbidity measurements (Träuble, 1971; Elamrani and Blume, 1983; Holzwarth and Rys, 1984), fluorescence techniques (Genz and Holzwarth, 1986; Genz et al., 1986; Holzwarth, 1989; Jørgensen et al., 2000; de Almeida et al., 2002), light and Raman scattering (Tsong, 1974; Tsong and Kanehisa, 1977; Yager and Peticolas, 1982), x-ray diffrac- 
tion (Cheng et al., 1994) and infrared spectroscopy (Jørgensen et al., 2000) were chosen. van Osdol et al. (1989) using volume perturbation calorimetry analyzed heat release or absorption and the rate of volume change accompanying a phase transition. Grabitz et al. (2002) monitored the time course of the answer of a calorimeter. These studies are complemented by ultrasonic methods (Mitaku and Date, 1982; Halstenberg et al., 2003) and dynamic heat capacity measurements (Yao et al., 1994; Nagano et al., 1995).

With the variety of methods employed the measured relaxation processes range from the $n s$ to the hour regime. Results have been analyzed using up to five different relaxation processes. Sometimes the monitored relaxation processes are not interpreted as a result of the phase transition of the lipid membrane but as an artefact of the experimental setup (van Osdol et al., 1989).

Genz and Holzwarth (1986) fit their data with five different relaxation times. Their relaxation times lie in the $1 \mathrm{~ns}, 100 \mathrm{~ns}, 1 \mu \mathrm{s}, 1 \mathrm{~ms}$ and $10 \mathrm{~ms}$ regime. The interpretation is that the lowest relaxation time represents the building of kinks in the fatty acid chains of the phospholipids, followed by a weakly cooperative process involving the free rotation of the head groups on the hexagonal lipid lattice. The third time is attributed to the formation of rotational isomers in the hydrocarbon chains. As it is the case with the remaining two relaxation processes the third process is highly cooperative. The last two times are attributed to the formation of the coexistence of different lipid domains. Grabitz et al. (2002) report one relaxation process. They yield results in the $s$-regime and relaxation times are as long as $45 s$ depending on the studied system. Jørgensen et al. (2000) and de Almeida et al. (2002) measure relaxation processes in two component lipid systems with a relaxation time of several hours. Biltonen and Ye (1993) describe two relaxation processes in binary lipid mixtures. The long times lie in the second regime. In the last four articles relaxation processes are related to domain formation processes in the lipid membrane.

Despite the fact that the setup, detection mode and details of the systems differ in each article most of the studies conclude that the kinetic slows down at the transition midpoint (Träuble, 1971; Tsong and Kanehisa, 1977; Mitaku and Date, 1982; Gruenewald et al., 1980; Elamrani and Blume, 1983; Holzwarth, 1989; van Osdol et al., 1991; Grabitz et al., 2002; Halstenberg et al., 2003). An exception is the work by Yager and Peticolas (1982). Cooperative processes govern most of the described relaxation behaviors. It has also been shown that cholesterol, which lowers the cooperativity of the transition, leads to a decrease in relaxation time (Träuble, 1971; Grabitz et al., 2002).

At first, theoretical approaches focused on cluster growth models (Tsong and Kanehisa, 1977). Recently, Grabitz et al. (2002) have suggested a non-equilibrium theory relating cooperative fluctuations in enthalpy with relaxation processes. They have assumed one relaxation process and have found that this one is proportional to excess heat capacity.

It can be summarized that different techniques have been used to study kinetic processes of the phase transition of lipid membrane systems. One has to take great care to compare these studies because of different observables and different underlying physical processes. All in all the studies have mainly confirmed that the kinetics are governed by cooperative processes with a slowing down in the transition regime. It can be speculated that this slowing down and the characteristic time scales have an implication on cell physiology. 


\subsection{Motivation}

A high diversity of different lipid species can be found in biological membranes. Currently, the reason for this is not fully understood. One can find bacteria like Escherichia Coli or Bacillus Stearothermophilus which adopt their lipid synthesize depending on growth temperature (Melchior and Steim, 1976; McElhaney and Souza, 1976; Jain and White, 1977; Pollakowski, 2003). This leads to the suggestion that lipids display a high importance in biological function. Therefore, it has been claimed that "not all the answers to biological function can be derived from the genome" (Mouritsen and Zuckermann, 2004) and the diversity of lipids are believed to play a major role. The decade of the lipids has been proclaimed (Hilgemann, 2003).

Lipid aggregates self-assemble on the macro-, nano- and meso-scale (Mouritsen and Jørgensen, 1995). Starting from the 1970s the importance of phase transitions and the formation of heterogeneities of pure lipid membranes has been formulated (Chapman, 1971; Träuble, 1971; Sackmann, 1984; Biltonen, 1990). Kinetic aspects have not been left out of these considerations (Träuble, 1971; Biltonen, 1990). It has taken, however, some time before domain formation processes in biomembranes have been considered as putatively being an important part in biological function. Considerations about this have become popular with the discussion about a highly specialized kind of domain, so called "rafts". They are believed to be enriched in cholesterol and sphingolipids (Brown and London, 1998; Simons and Vaz, 2004). Since their introduction a lot of research has focused on their implications and importance in trafficking processes in biological cells (Brown and London, 1998; Simons and Vaz, 2004; Brown and London, 2000; Mukherjee and Maxfield, 2000; Ikonen, 2001; Bodin et al., 2003; Matteis and Godi, 2004; Helms and Zurzolo, 2004). Still, it is surprising that the focus lies on these kinds of domains. Domain formation processes in general can have an influence on diffusion properties (Vaz et al., 1989; Saxton, 1999) and protein activity (Kamp et al., 1974, 1975; Gabriel et al., 1987; Lichtenberg et al., 1986; Grainger et al., 1989; Burack and Biltonen, 1994; Bolen and Sando, 1992; Dibble et al., 1996; Hønger et al., 1997; Hinderliter et al., 2004). Inhomogeneities also influence in-plane reactions and therein protein activity. That might act as an on- and off-switch (Melo et al., 1992; Vaz and Almeida, 1993; Thompson et al., 1995; Salinas et al., 2005).

Lipids in general have an influence on the activity of proteins, as was discussed a few years after the publication of the fluid mosaic model (Sandermann, 1978). Changes in lipid composition might induce cell dysfunction and lead to diseases (Bienvenüe and Marie, 1994; Peet et al., 1999). The activity and function of proteins can depend on lipid chain length (Caffrey and Feigenson, 1981; Brown, 1994) or mechanical properties (Brown, 1994; Wiggins and Phillips, 2005). Proteins might show a higher activity in a "ld" than in a "so" environment (Lee, 2003, 2004).

In the beginning these effects were attributed to the fluidity ${ }^{17}$ of a membrane. There has, however, been evidence against the triggering of protein activity and function by fluidity (Lee, 1991). Hydrophobic matching (Mouritsen and Bloom (1984); see sect. 1.1.3) of the lipid bilayer and the protein is widely accepted as influencing protein function (Dumas et al., 1999; Jensen and Mouritsen, 2004). It has also been suggested that the lateral pressure profile through a membrane bilayer affects protein conformation and therewith function (Cantor, 1999a,b). Thermodynamical fluctuations in density and lateral composition are also discussed

\footnotetext{
${ }^{17}$ The term fluidity has never gotten a clear definition. It refers to the lateral disorder of the lipid membrane.
} 
in the context of protein function (Biltonen, 1990; Mouritsen and Jørgensen, 1995). Fluctuations, however, might also influence cell function because of influences on passive permeation properties of a membrane (Cruzeiro-Hansson and Mouritsen, 1988; Makarov, 2005).

For a complete understanding of biological function on the cell level an understanding of lipid-lipid, lipid-protein and protein-protein interactions is necessary. In a biological membrane all these interactions lead to an inhomogeneous system with domains of a different nature (Parsegian, 1995). Lipid domain formation putatively plays an important role in the control of biological function. Therefore, an understanding of domain formation processes is necessary. Heterogeneities influence the diffusion properties of lipids themselves and those of proteins too. Relaxation phenomena determine the life time of domains and influence the time scale of domain formation processes after a perturbation. The latter one gains importance since "There is nothing so dead as a system at equilibrium." (Parsegian in Edidin (1992)). Fluctuations are an intrinsic property of model, as well as biological membranes. Fluctuation time scales might determine characteristic time scales in biological function.

This thesis is a combination of experimental and simulation techniques. Diffusion, relaxation, domain formation and fluctuation properties and their relation to each other are investigated in the proximity of melting transitions of artificial membranes. Diffusion measurements are based on fluorescence correlation spectroscopy ${ }^{18}$ and pressure perturbation calorimetry is applied for the evaluation of relaxation processes in model membranes ${ }^{19}$. These experimental results are accompanied by the evaluation of a thermodynamical model using numerical simulations. Domain formation and fluctuation properties are further studied using the statistical thermodynamics simulations. The validity of the results can be indirectly deduced from measured excess heat capacity profiles.

In the proceeding chapter the experimental techniques and procedures will be introduced. This is followed by a chapter about the theoretical aspects of this work. In chapter 4 some results are presented that are later discussed in the final chapter which also includes remarks on a possible role of the findings in the physiology of cells. Suggestions about further research directions and perspectives are also given.

\footnotetext{
$1 \overline{18}$ Experiments were performed by A. Hac; Hac (2004).

${ }^{19}$ A series of measurements on DMPC/Gramicidin A systems was done by M. Gudmundsson; Gudmundsson (2004).
} 


\section{Chapter 2}

\section{Material and Methods}

In this chapter the experimental methods and the sample preparation are explained. Results from different experimental techniques are presented in this thesis. These techniques are mainly Differential Scanning Calorimetry (DSC), Pressure Perturbation Calorimetry (PPC) and Fluorescence Correlation Spectroscopy (FCS). The principal construction of a chip calorimeter is also explained. The numerical model which accompanies the experimental work is not mentioned in this chapter, but is explained in a separate chapter (see chapter 3.1.2).

\subsection{Differential Scanning Calorimetry}

Differential Scanning Calorimetry (DSC) is a powerful experimental technique which allows one to study endothermic or exothermic processes in a large variety of different materials or solutions. Since the late 1960s DSC was used for the study of melting processes in artificial or biological membrane systems (Steim et al., 1969; Reinert and Steim, 1970; Hinz and Sturtevant, 1972; Melchior and Steim, 1976; Mabrey and Sturtevant, 1976; Jackson and Sturtevant, 1977; Blume, 1983; Heimburg and Biltonen, 1996; Ebel et al., 2001; Pollakowski, 2003; Heerklotz, 2004). It is also used for the study of protein denaturation (Privalov et al., 1986; Plotnikov et al., 1997). In this work DSC is used for the measurement of heat capacity profiles of different lipid membrane model systems. This includes simple one component lipid systems, binary lipid systems or lipid membranes with added peptides or neurotransmitters or anesthetics. For these measurements we mainly used the VP-DSC from Microcal (Northhampton/MA, USA; (Plotnikov et al., 1997)). A few measurements were performed on the MSC from Microcal (Northhampton/MA, USA). The principal of both calorimeters is the same and the difference lies in the technical realization and sensitivity. The VP-DSC is the more modern one and is one of the most sensitive DSCs on the market.

The principal of a DSC is very simple (see fig. 2.1). A DSC consists of two cells which are surrounded by an adiabatic shield. One of the two cells is filled with a reference and the other one is filled with a sample substance. In our case the reference cell is usually filled with a buffer and the sample cell with a lipid/buffer solution. The temperature of the cells is changed with a constant scan rate and the temperature difference between the two cells is kept at zero. If processes happen in one cell which do not happen in the other, the calorimeter requires a different power to heat or cool the reference cell than it does the sample cell. This power difference does not equal zero $\left(\Delta P=P_{\text {sample }}-P_{\text {reference }} \neq 0\right)$. One can show that the 


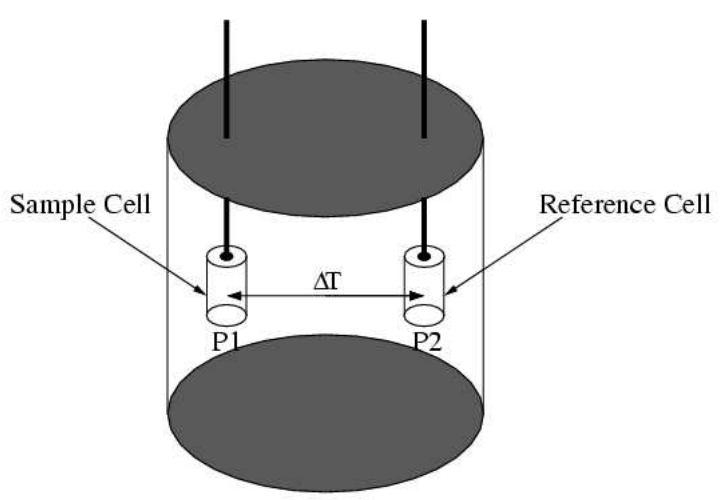

Figure 2.1: Schematic drawing of a differential scanning calorimeter. A sample and a reference cell are protected from the outer environment through an adiabatic shield. The temperature difference between the two cells $\Delta T$ is kept zero. The difference between the powers which are needed to heat each of the cells is proportional to the excess heat capacity.

measured power difference and the excess heat capacity are proportional to each other.

For a short time interval $\Delta t$ one gets for the heat difference $\Delta Q$ :

$$
\Delta Q=\int_{t}^{t+\Delta t} \Delta P d t \approx \Delta P \Delta t
$$

Using basic thermodynamics one finds:

$$
\left(\frac{d H}{d T}\right)_{\mathrm{p}}=\Delta c_{\mathrm{p}}=\left(\frac{\partial Q}{\partial T}\right)_{p} \approx \frac{\Delta Q}{\Delta T} \approx \frac{\Delta P}{\frac{\Delta T}{\Delta t}},
$$

where $\mathrm{H}$ is the enthalpy and $\Delta T / \Delta t$ is the scan rate.

This means that by measuring the power difference of the two cells one has a direct measure of the excess heat capacity of the sample substance, in our case of the lipid model system.

The calorimeters of MicroCal allow one to define the starting and ending temperature plus the scan rate. With the VP-DSC one can also adjust the integration time and the feedback mode of the calorimeter. The integration time gives the time interval in which calorimetric data is collected and averaged. The calorimeter reacts with the highest possible response time if the feedback is set to be high, but the sensitivity is decreased in comparison to a low feedback. The heat capacity measurements were performed at different scan rates, using a high feedback and a $10 \mathrm{~s}$ integration time in all cases.

\subsection{Pressure Perturbation Calorimetry}

One of the aims of this thesis is the thermodynamical study of kinetic properties of artificial membrane systems. Therefore, one needs to find possible methods in perturbing the membrane system. Fig. 2.2 shows DSC scans of a dispersion of multilamellar DMPC vesicles at different pressures. A higher pressure leads to a shift of the melting profile to a higher temperature. However, the shape is not changed. An additional pressure of $\Delta P=40$ bar results 
in a shift of $\Delta T=0.95 \mathrm{~K}$. Multilamellar vesicles show a highly cooperative melting transition, meaning that the half width of the $\Delta \mathrm{c}_{\mathrm{p}}$-profile is small $(\approx 0.1 \mathrm{~K})$. Therefore, the two profiles measured at ambient pressure and at an increased pressure of 41 bar do not intersect.

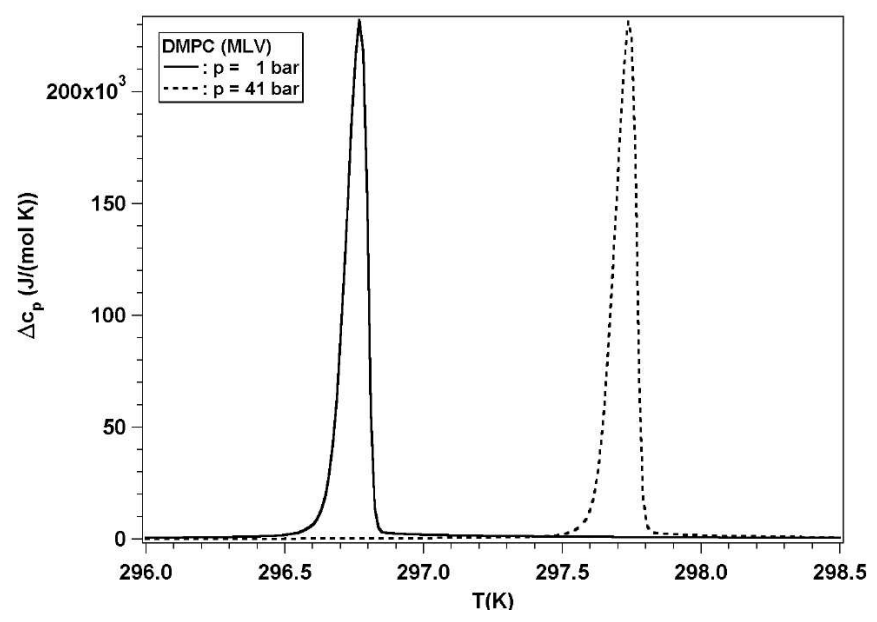

Figure 2.2: Heat capacity profiles of multilamellar DMPC vesicles at ambient pressure and at an increased pressure of $41 \mathrm{bar}$. One can clearly recognize that a bigger pressure leads to melting at higher temperatures. The shape of the transition profile does not change.

This pressure dependence can be used to jump into or out of the transition regime. One possibility is to adjust the temperature so that with additional pressure the membrane is in its "so" phase, whereas domain coexistence is present at atmospheric pressure. This means that by putting the lipid system under an additional pressure one can jump into the transition regime when releasing the pressure. In the other case the temperature is set so that the lipid membrane displays a domain coexistence if pressure is added to the system. Then increasing of pressure means that one jumps from the "ld" phase into the melting regime.

Here, we are interested in relaxation processes after a jump into the transition regime. Depending on whether one jumps from above or below the transition regime the membrane system releases or absorbs heat. The heat is taken from, or released into, the surrounding water. This again shall be compensated by the calorimeter which tries to keep the sample's temperature constant.

In the investigation of the relaxation processes we use two different methods. One method assumes that the calorimeter can keep the sample's temperature constant and the calorimeter response on a pressure jump is analyzed (Method I). The second method measures a temperature change in the surrounding water, since it is assumed that the idealized situation of no temperature change at all times cannot be achieved experimentally (Method II). In the following sections both methods are explained.

\subsubsection{Method I}

The Microcal VP-DSC gives one the possibility to operate it in a scanning or isothermal mode. The isothermal mode allows one to keep the calorimeter cells at a constant temperature and to monitor the power difference of the heaters of the two calorimeter cells as a function of 
time. One can set the integration time and the feedback of the VP-DSC calorimeter. For the pressure jump experiments the integration time is chosen to be $1 s$ and a high feedback is adjusted.

A self-built pressure cell which can hold a pressure of up to $200 \mathrm{bar}$ is filled with the lipid sample. It is put into the sample cell of the calorimeter. The sample and reference cell of the calorimeter are filled with millipore water. The pressure cell is connected to a nitrogen pressure bottle through a system of hoses. A pressure reducer (Messer Griesheim, Frankfurt, Germany) allows one to adjust the maximum additional pressure onto the lipid system from 5-200bar. Manual valves (Nova Swiss, Effretikon, Switzerland) are used to control a pressure increase or release. The additional pressure applied on the system through the nitrogen is measured with a pressure gage (Nova Swiss, Effretikon, Switzerland). The time evolution of the pressure increase or release is well described with an one component exponential fit. The characteristic time scales are faster than $200 \mathrm{~ms}$. This is much faster than the time resolution of the calorimeter.

After a pressure jump the calorimeter response results in a characteristic curve like it is shown in fig. 2.3. In this figure two calorimeter responses are shown. One after a negative pressure jump ( $n p j)$ when the additional pressure is released and another one after a positive pressure jump ( $p p j$ ) when pressure on the system is suddenly increased. In this case (fig. 2.3) the temperature was chosen so that one jumps into the transition regime with a $n p j$ and out of it with a $p p j$.

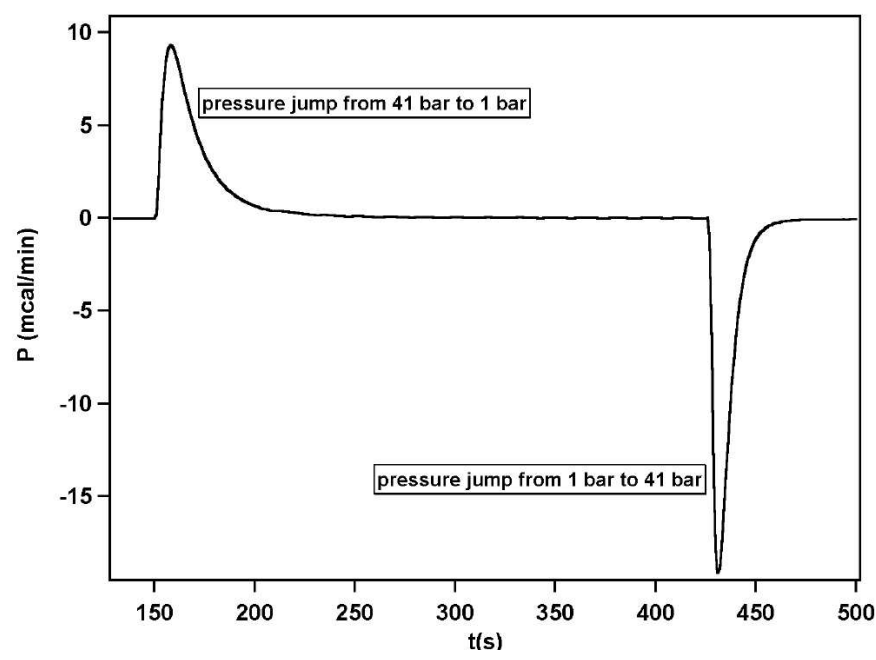

Figure 2.3: Example calorimeter responses after a pressure jump at constant temperature. In the displayed example a sudden decrease in pressure results in a jump into the transition regime. With increasing pressure one jumps out of the melting regime. The area under the curves of the response after a negative pressure jump $(n p j)$ and a positive one $(p p j)$ are the same. From the difference in amplitude one can qualitatively deduce different relaxation behaviors. The relaxation time in the first case is higher than in the second one.

One notices the different amplitudes of the two signals. The area below both curves must be equal which means that for the $n p j$ the relaxation process is longer than for the $p p j$. The calorimeter response gives us a measure of the characteristic time scales of the relaxation processes. 

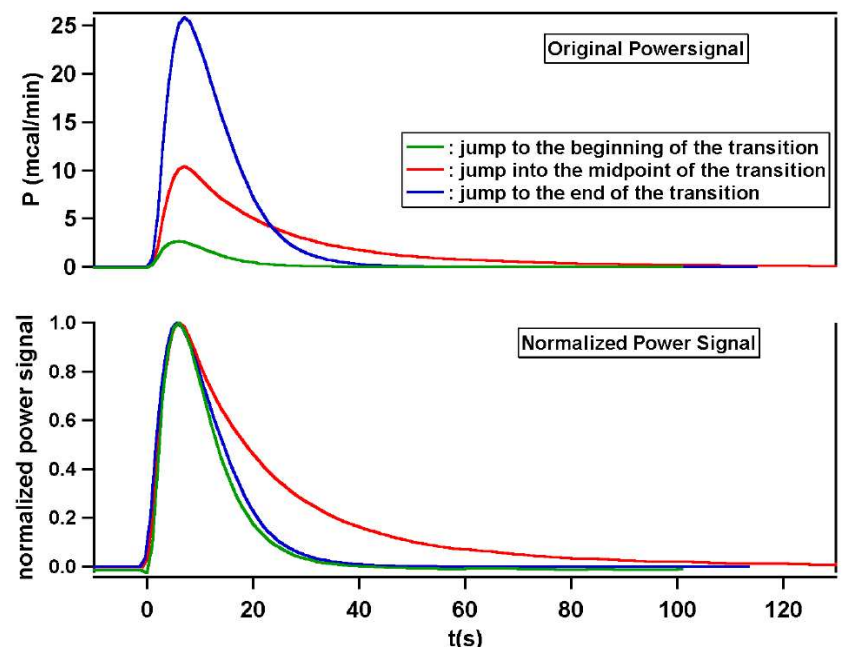

Figure 2.4: Examples of calorimeter responses when jumping to different regions of the transition. Jumping to different points of the transition result in different calorimeter responses. The area below the curves, as well the relaxation times differ.

In fig. 2.4 npj examples have been selected to show jumping to different parts of the transition. One example depicts the case of a jump to the beginning of the transition, another to the end and lastly one to the midpoint of the transition, wherein the excess heat capacity is at its maximum. The areas below the curves differ and the more one jumps through the transition the bigger the areas get. A normalization of the curves' amplitudes allows one to compare the time scales of the responses. One clearly sees that the jump to the midpoint results in a higher relaxation time than the jumps to the beginning or the end of the transition. In the latter two cases the relaxation times are about the same.

Grabitz et al. (2002) have developed a model to analyze the calorimeter response after such pressure jumps. We have adopted this approach for the evaluation of our data. This model is based on the assumption that the response of the calorimeter is a convolution of the lipid signal with the instrument response.

The latter one is a result of the experimental setup and originates from the response of the water and the pressure cell walls:

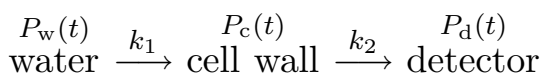

The instrument response can be experimentally gained through pressure jump experiments on pure water samples or through pressure jumps which are far away from the transition regime. In the latter case, there is no contribution from the lipids since the lipid membranes do not change their phase state. This is equivalent to the case with pure water. In these cases it is assumed that there is only a need for a minor release, or absorption, of heat. This can be described as a $\delta$-pulse. In the case of heat release, heat is transfered to the cell wall with a rate constant $k_{1}$ and then from the cell walls to the detector with a rate constant of $k_{2}$. The functions $P_{\mathrm{w}}(t), P_{\mathrm{c}}(t)$ and $P_{\mathrm{d}}(t)$ are time dependent functions of the heat in the water, 
in the cell wall and the detector. This leads to the following coupled differential equations:

$$
\begin{aligned}
\frac{d P_{\mathrm{w}}(t)}{d t} & =-k_{1} P_{\mathrm{w}}(t) \\
\frac{d P_{\mathrm{c}}(\mathrm{t})}{d \mathrm{t}} & =k_{1} P_{\mathrm{w}(\mathrm{t})}-k_{2} \mathrm{P}_{\mathrm{c}}(\mathrm{t}) \\
\frac{d P_{\mathrm{d}}(t)}{d t} & =k_{2} P_{\mathrm{c}}(t),
\end{aligned}
$$

with $\mathrm{P}_{\mathrm{w}}(0) \neq 0$ and $\mathrm{P}_{\mathrm{c}}(0)=0$. Using method I one evaluates the calorimeter response which is described by $\mathrm{P}_{\mathrm{d}}(\mathrm{t})$. Solving the three differential equations (eq. 2.5) one finds:

$$
P_{\mathrm{d}}(t)=P_{\mathrm{d}}^{0}\left(e^{-\mathrm{k}_{2} \mathrm{t}}-e^{\mathrm{k}_{1} \mathrm{t}}\right) .
$$

This solution can be used for the definition of a normalized instrument response function:

$$
\begin{aligned}
R_{\text {inst }}(\mathrm{t}) & =\left(\frac{k_{1} k_{2}}{k_{1}+k_{2}}\right)\left(e^{-k_{2} t}-e^{-k_{1} t}\right) & & t \geq 0 \\
R_{\text {inst }}(t) & = & 0 & \\
\int_{0}^{\infty} R_{\text {inst }}(t) d t & = & 1 &
\end{aligned}
$$

Eq. 2.5 is the solution for the instrument response as it is expected after a jump far away from the melting transition or after a jump using a pure water sample. The values for $k_{1}$ and $k_{2}$ can be gained through fitting this curve to experimental results.

It has already been mentioned that the calorimeter response after a jump into or out of the transition regime is a convolution of the lipid signal with the instrument response. After a pressure jump the membrane system releases or absorbs heat and the relaxation process dominates the calorimeter response with respect to the perturbation signal described by the instrument response. The scheme from eq. 2.3 can be modified:

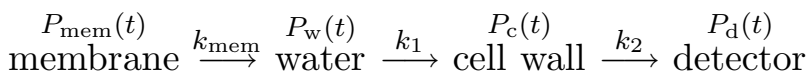

It is assumed that the relaxation process of the membrane system can be described by a single exponential decay (see also sec. 3.2) and therefore, the heat release or absorption of the lipid membrane can be written as:

$$
\begin{array}{ll}
P_{\mathrm{mem}}(t)=P_{\mathrm{mem}}^{0} e^{-k_{\mathrm{mem}} t} & t \geq 0 \\
P_{\mathrm{mem}}(t)=0 & t<0 .
\end{array}
$$

The monitored calorimeter response can then be described by:

$$
P_{\text {cal }}(t)=\int_{0}^{t} P_{\text {mem }}(\tau) R_{\text {inst }}(t-\tau) d \tau+P_{d}(t)
$$

This convolution process allows one to increase the time resolution of the experiment. In the case of a fit of the decreasing part through an exponential decay, the time resolution is limited to about $5 \mathrm{~s}$. Using the convolution process one reaches a time resolution of approximately $3-4 s$ (Grabitz et al., 2002). This time resolution, however, is still not high enough for our purposes. Therefore, we have developed a second method to evaluate relaxation times. This method is based on measuring the temperature change of the sample after a pressure jump and analyzing its time course. This new method is explained in the following section. 


\subsubsection{Method II}

As explained in the previous section relaxation times after a pressure jump can be evaluated using the response of the VP-DSC calorimeter in the isothermal mode. This yields a time resolution in the second time regime. Grabitz et al. (2002) argue that relaxation times are proportional to heat capacity. Relaxation times of lipid systems with decreased cooperativity, therefore, are assumed to have fast relaxation processes that are difficult to resolve with the previously introduced method. Large unilamellar vesicles (LUVs; diameter of about $100 \mathrm{~nm}$ ) such as DPPC are example systems which display a decreased cooperativity and fast relaxation processes. For DPPC LUVs Grabitz et al. (2002) measured a maximum relaxation time of about $3 \mathrm{~s}$. The data showed high scattering because relaxation times where at the resolution limit. It is estimated that biological membranes have relaxation times in the $10-100 \mathrm{~ms}$ regime (Grabitz et al., 2002). Finding a method that yields a higher resolution but still measures relaxation times by thermodynamical means might be a step further towards the study of relaxation phenomena on biomembranes. The situation that the temperature after a pressure jump stays constant is idealized. Measuring the temperature change of the sample after a pressure jump seems to be a promising approach.

\section{Setup: Mechanical Parts}

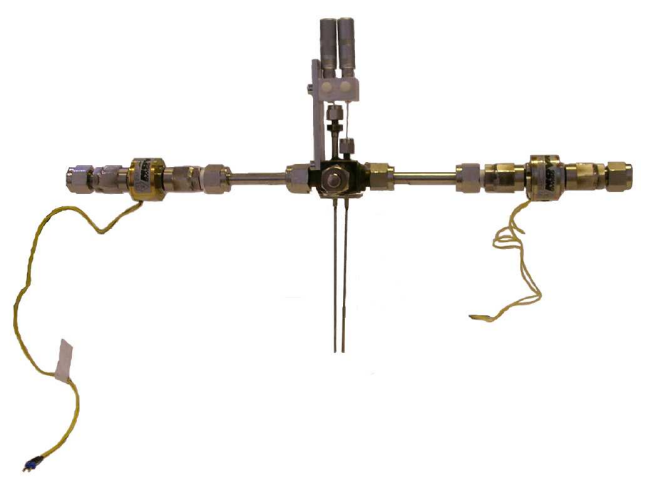

Figure 2.5: Pressure cell for method II. A pressure cell with two capillaries has been built. Thermocouples can be let into the two capillaries. Changes in temperature after a pressure jump can be monitored with these. Pressure release or increase is realized with computer controlled coaxial solenoid valves.

For this purpose a pressure cell which can hold a pressure of about $80 \mathrm{bar}$ has been constructed (see fig. 2.5). This pressure cell has two capillaries instead of just one unlike the one which was used in "method I". One is for the reference and the other is for the sample substance. It exactly fits into the two calorimeter cells of the VP-DSC.

For the control of the pressure release or addition two coaxial solenoid valves (Nova Swiss, Effretikon, Switzerland) are connected to the pressure cell. They have maximum opening and closing times of $20 \mathrm{~ms}$. One of these valves is connected to a pressure reducer (Messer Griesheim, Frankfurt, Germany). This again is connected to a nitrogen bottle with a maximum pressure of $200 \mathrm{bar}$ and allows one to adjust between pressures of 5 and $200 \mathrm{bar}$.

A self-constructed Microsoft Visual C++ program controls a power supply which permits one to open (current on) or close (current off) each of the valves. Due to a high increase in 
the temperature of the valves whilst in the open state, the valves are not allowed to be open for more than $60 \mathrm{~s}$.

Opening the valve which allows the nitrogen to enter the cell (valve-in) results in a pressure increase with a time constant of $90 \mathrm{~ms}$. The pressure release can be described with a time constant of $250 \mathrm{~ms}$. The latter pressure vs. time curves can be very well fitted with a single exponential function, whereas the first one shows some deviations. The time constant of $90 \mathrm{~ms}$ sets an upper limit. This deviation is probably due to the function of the pressure reducer.

The used valves show a high sensitivity to impurities in the setup (personal communication with Nova Swiss). Even though the nitrogen used has a high purity it is still not pure enough to meet to the valves requirements. The valve-in has gotten soiled so that it does not perfectly close any more. The valve-in leaks, meaning that in the case when the pressure has been released and the valve-out has been closed again (closing after $30 \mathrm{~s}$ ), the pressure in the cell increases with time. This process, however, is rather slow (approx. $+0.1 \frac{\mathrm{bar}}{\mathrm{min}}$ ). On the other hand if the pressure in the cell is increased to 41 bar, leaks at the screwings of the pressure cell lead to a decrease in pressure. Again, the process is slow (approx. $-0.1 \frac{\mathrm{bar}}{\mathrm{min}}$ ). Both leak processes are assumed not to distort the relaxation time measurements.

\section{Setup: Electronic Parts}

After a pressure jump the temperature evolution in the sample capillary is monitored ${ }^{1}$. Thermocouples show a high time resolution in measuring temperature changes. Thermocouples were bought from Thermocoax (Stapelfeld, Germany). The diameter of a thermocouple cable is $0.25 \mathrm{~mm}$. Each thermocouple cable is a combination of the two thermocouple conductors, an insulation and a metal protecting sheath. For our experiments so called Kthermocouples are used. This means that one of the two conduction wires is made of the alloy chromel (nickel-chromium) and the other one of alumel (nickel-aluminum). The advantage of $\mathrm{K}$-thermocouples is that in the temperature regime of interest they show a constant sensitivity of $41 \frac{\mu V}{K}$. A version of the thermocouples has been chosen were the cores of the chromel and alumel wires are welded to the sheath. This allows a fast response time. It is faster than in the case where the connection of the two conductors lies in the insulation. The intrinsic response time ${ }^{2}$ is about $7 \mathrm{~ms}$.

The thermocouple signal is amplified by a nanovolt amplifier (Nanovolt Preamp Model 1801, Keithley Instruments Inc., Cleveland/OH, USA) and is monitored by a multimeter Keithley Model 2001 (Keithley Instruments Inc., Cleveland/OH, USA). The nanovolt amplifier, the connections of the compensating cables with the thermocouples and the contacts between the compensating cable wires of the thermocouples and the copper wires of the nanovolt amplifier are thermally isolated from their environment. This is necessary in order to prevent slow but strong fluctuations in the mean thermocouple signal without perturbation of the

\footnotetext{
${ }^{1}$ The actual idea was monitoring the differences of the two thermocouple signals in the reference and sample cell. The signal was influenced by mechanical perturbations which result after opening of one of the valves. The thermocouple signal was perturbed and did not show any response from the lipid sample. It could not be used for the evaluation of relaxation processes. The reason of these problems could not be found and solved. Because of differences of the two capillaries and of the used thermocouples it seems anyways questionable whether a direct monitoring of the difference would have been possible. Measuring the voltages of the single thermocouples in pure water after each other showed that the signals had different voltage offsets. The amplitudes of the signals after a pressure jump differed, too.

${ }^{2}$ The intrinsic response time is the time needed for the heat to flow through the surrounding medium and the thermometer.
} 


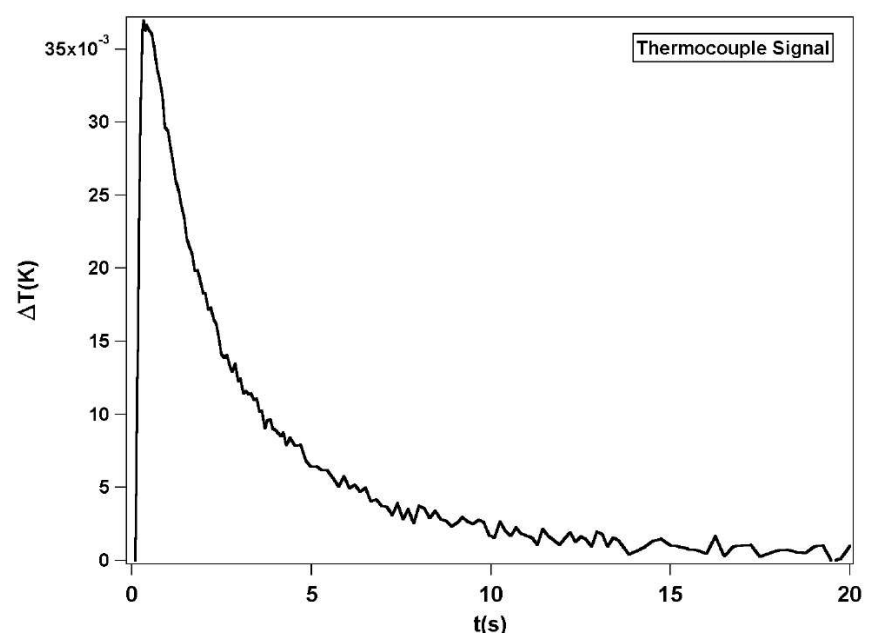

Figure 2.6: Example thermocouple signal after a positive pressure jump. The pressure on the system was increased. The temperature was chosen so that one jumped from the "ld" phase into the domain coexistence region. It can be seen that the temperature increases. The signal is a contribution from a perturbation signal and a contribution originating from the lipid membrane. Jumping from the "ld" phase into the coexistence regime means that some lipids freeze again, which is an exothermic process. Therefore, the temperature of the surrounding water increases.

system. The nanovolt amplifier has to be kept at least $1 m$ away from possible strong sources of electromagnetic waves. These can perturb the signal. The measured data can be read out from the multimeter with a Keithley GPIB card PCI-488 (Keithley Instruments Inc., Cleveland/OH, USA). This card also allows the control of the parameters of the multimeter as such as integration time, time interval between measurement points and voltage range. The nanovolt amplifier allows three voltage settings (maximal voltage: $2 \mathrm{mV}, 200 \mu \mathrm{V}$ and $20 \mu V)$. The best resolution is achieved on the maximal voltage setting of $20 \mu \mathrm{V}$. This one cannot be used since the voltage measured before the pressure jump is usually higher than $20 \mu V$. Actually, one would expect to obtain the temperature in the pressure cell from this voltage. However, this is not true because contact voltages in the connections between the compensating cables and the copper wires lead to voltage offsets. This offset is constant and therefore, does not play a role when studying the time course of the voltage or temperature signal. To avoid problems, a maximum voltage of $2 m V$ has been chosen. This resolution was sufficient in all our experiments. Times between measurements varied throughout the recording so that long data traces could be monitored. The integration time was set to auto. The multimeter adjusts the most optimal integration times in dependence on the times between successive data points itself. The time resolution of the calorimeter is increased by turning off the LCD display and disabling the autozeroing process of the multimeter during a measurement. This is crucial at the beginning of the temperature monitoring since all running processes of the multimeter lead to a degraded time resolution. At first, signals are recorded every $25 \mathrm{~ms}$. This time interval is increased a few times during the measurement. This is necessary because the internal storage buffer of the multimeter is limited to 401 data points. Therefore, it is not possible to make measurements up to a few minutes with a time of $25 \mathrm{~ms}$ between each recording. 
In the experiment the VP-DSC calorimeter is used as a water bath. It is run in the isothermal mode with a high feedback and an integration time of $1 s$.

\section{Temperature Course and Fitting of the Response}

An example of a temperature change after a pressure jump is plotted in fig. 2.6. The measured curves show an offset, which was corrected. The thermocouple measures voltages, which are related to temperatures. As mentioned the sensitivity of the used thermocouples is $41 \frac{\mu V}{K}$. In the case shown the temperature and the pressure were chosen so that a jump into the coexistence regime of a lipid membrane was made. Before the jump the lipid membrane was in the "ld" state. During the domain formation process some lipids undergo a freezing process. This is an exothermic process. The produced heat is transfered to the water so that the temperature increases. Jumping from the "so" phase into the coexistence regime results in a decrease of temperature (data not shown).

Performing pressure jump experiments at temperatures which are far away from the melting transition regime or in a pure water environment also result in changes of temperature (data not shown). These signals are a result of an answer of the water, the pressure cell and its capillaries with their construction differences. The signal is temperature dependent, which is probably due to a change in water volume. This effect was recently used to measure the temperature dependence of thermal expansion coefficients for different solutes (Lin et al., 2002). The amplitude of the temperature change is temperature dependent and increases with growing temperature. In the following this signal is addressed as the perturbation signal. An expression for this perturbation signal shall be formulated in the following.

Changing pressure results in a perturbation which induces a heat change $\Delta Q_{\text {per }}=c_{p} \cdot \Delta T_{\text {per }}$. This one induces a change in temperature which depends on the heat capacity of the setup. The heat capacity is assumed to be constant, so that considering heat or temperature changes is equivalent. The water temperature changes depending on the rate constant $k_{1}$ of the perturbation and on the rate constant $k_{2}$ of the temperature compensation due to the coupling to a water bath (the calorimeter). This can be described with the following scheme:

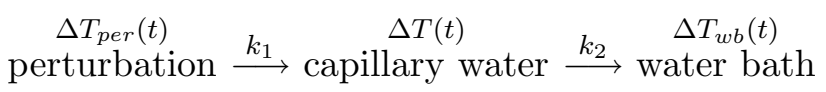

This model leads to coupled differential equations similar to eq. 2.5:

$$
\begin{aligned}
\frac{d \Delta T_{\mathrm{per}}(t)}{d t} & =-k_{1} \Delta T_{\mathrm{per}}(t) \\
\frac{d \Delta T(t)}{d t} & =k_{1} \Delta T_{\mathrm{per}}(t)-k_{2} \Delta T(t) .
\end{aligned}
$$

The thermocouple measures the temperature of the capillary water. At long times the temperature change is zero. For the time dependence of the water temperature one finds:

$$
\Delta T(t)=T_{0,2} e^{-k_{2} t}-T_{0, \text { per }} e^{-k_{1} t},
$$

where $T_{0,2}$ and $T_{0, \text { per }}$ are respective amplitudes.

It has been phenomenologically found that for the description of the perturbation signal the assumption of two processes which lead to a temperature compensation result in a better 
agreement of fit and actual signal. This has also been verified performing pressure jump experiments on systems where the two pressure cell capillaries were either filled with ethanol (denatured; VWR International, Roskilde, Denmark) or ethylene glycol (Fluka, Buchs, Switzerland). This means that there are two processes which couple the temperature of the capillary water to the water bath. One process happens on the $300 \mathrm{~ms}$, whereas the other on the $3 \mathrm{~s}$ time scale. The reason why there are two processes, is not understood.

The expression for the perturbation signal equals then:

$$
\Delta T(t)=T_{0,2} e^{-k_{2} t}+T_{0,3} e^{-k_{3} t}-T_{0, \mathrm{per}} e^{-k_{1} t},
$$

where $T_{0,2}, T_{0,3}$ and $T_{0, \text { per }}$ are respective amplitudes.

From this we can define the instrument response:

$$
\begin{array}{ll}
R_{\text {inst, } \mathrm{T}}(t)=\frac{T_{0,2}}{T_{0,3}} e^{-k_{2} t}+e^{-k_{3} t} \quad t \geq 0 & \\
R_{\text {inst }, \mathrm{T}}(t)=0 & t<0,
\end{array}
$$

where the factor $\frac{T_{0,2}}{T_{0,3}}$ weights the two processes which lead to a temperature compensation.

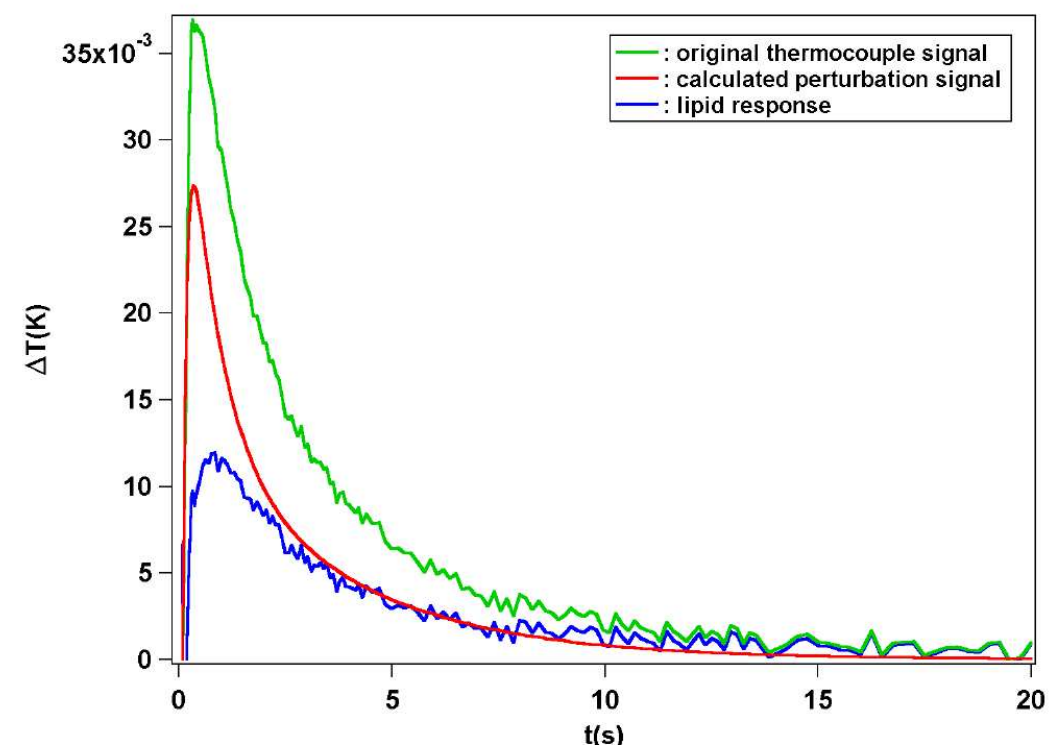

Figure 2.7: Thermocouple Signal: Original, perturbation and lipid signal. The temperature change as it is measured with the thermocouple (green curve) is a result of a perturbation signal (red curve) and the signal as it is influenced by the relaxation kinetics of the lipid sample (blue curve). The perturbation signal originates from the water, the pressure cell and the imperfections of the two pressure cell capillaries.

In all performed experiments with the lipid/water systems perturbation signals were measured at six different temperatures, three well above and three well below the temperatures of 
the transition regime. Fitting the responses ${ }^{3}$ with eq. 2.14 allows an estimation of the average values for $k_{1}, k_{2}$ and $k_{3}$. $T_{0, \text { per }}, T_{0,2}$ and $T_{0,3}$ are temperature dependent. From the dependence found one can calculate values for $T_{0, \text { per }}, T_{0,2}$ and $T_{0,3}$ at the transition temperature. The perturbation signal is assumed to be constant over a short temperature interval.

Performing pressure jump experiments into the transition regime one gets a signal which depends on the perturbation signal and the signal which originates from the lipids. With the estimation of the perturbation signal one has the possibility to extract the lipid response from the whole signal.

In fig. 2.7 an example of a thermocouple signal, the corresponding perturbation signal and the lipid response are plotted. Since the perturbation is calculated with the analytic eq. 2.14 one does not find noise in the curve. Subtracting the perturbation signal from the original response allows one to fit only the lipid response. The advantage of this is explained below and is visualized in fig. 2.8.

The lipid response depends on the time scale of the relaxation process and the heat transport rates $k_{2}$ and $k_{3}$. It is assumed that the relaxation process can be described by a single exponential decay $\left(e^{-k_{\text {lipid }} t}\right.$, with $\left.\tau_{\text {lipid }}=\frac{1}{k_{\text {lipid }}}\right)$. The temperature development is seen as a convolution of the lipid response with the instrument response:

$$
\Delta T_{\text {lipid }}(t)=T_{0}^{l i p i d} \int_{\tau=0}^{t} e^{-k_{\text {lipid }} \tau} R_{\text {inst }, \mathrm{T}}(t-\tau) d \tau,
$$

where $T_{0}^{l i p i d}$ depends on the total heat production or absorption of the lipid membrane after a pressure jump.

Fig. 2.8 is a representation of the behavior of the thermocouple signal at different temperatures after an increase of pressure. These results were gained after measurements on a suspension of $40 \mathrm{mM}$ DMPC plus $20 w t \%$ halothane.

The thermocouple signal is a voltage. Using the sensitivity of the K-type thermocouple one can calculate temperature changes out of it. The original, baseline corrected thermocouple signal and the lipid response are plotted. Each time in absolute values and normalized ${ }^{4}$. The perturbation signal $(T=297.546 \mathrm{~K})$ is drawn in order to compare it with the signals after jumps into the transition. If one jumps from the "ld" phase into the melting regime heat is released. Here, the heat capacity maximum is at $298.080 \mathrm{~K}$. The farther one jumps into the transition the more heat is released. This means that at lower temperatures a bigger heat release is observed. The maximum temperature change depends not only on the heat release but also on the relaxation behavior of the lipid membrane. In the upper, left panel of fig. 2.8 it can be seen that in the shown examples the maximum temperature alteration is the highest at the lowest temperature. This lowest temperature was chosen to lie at the lower temperature limit of the transition. The smallest temperature perturbation is as expected after a jump outside of the transition regime. In the lower, left panel the absolute values of the temperature changes as a result of the lipid response are plotted. They were obtained after a subtraction of the perturbation from the total thermocouple signal. Often these changes are smaller than the ones induced through the perturbation. This depends on the point of transition one jumps to and the lipid concentration.

\footnotetext{
${ }^{3}$ Before the signal can be fitted, a correction of the thermocouple signal is done. This is necessary because the thermocouple signal is not ideal and usually the voltage does not go back to the value before the jump. This is corrected with fitting a baseline. This also allows to correct the curve so that the start and end voltage fluctuates around $0 \mathrm{~V}$.

${ }^{4}$ All amplitudes are normalized to one.
} 

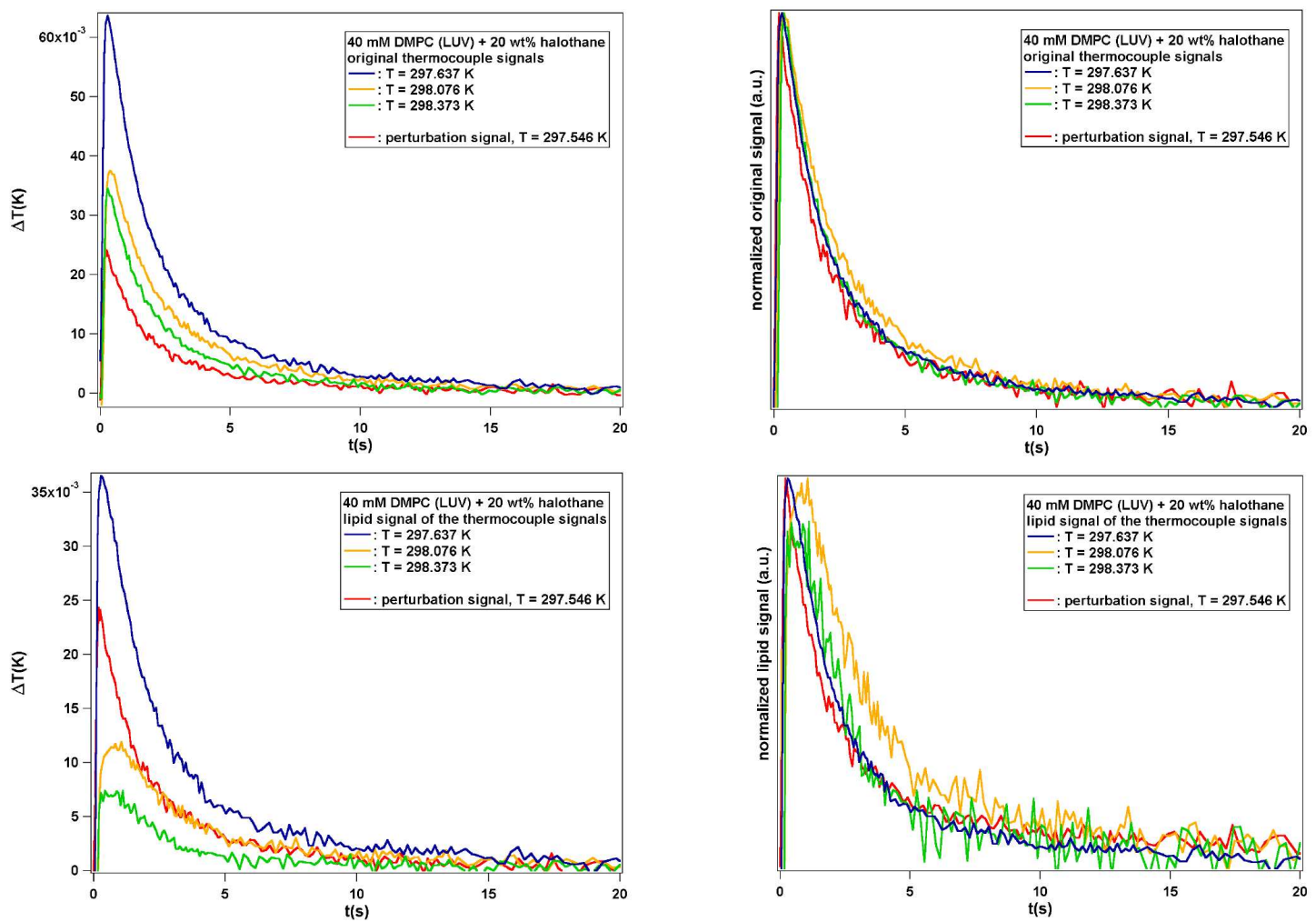

Figure 2.8: Original and lipid signals at different temperatures. Positive Pressure Jumps were performed at different temperatures. One jumps into different regions of the melting transition. Therefore, the relaxation behavior differs. This is reflected in the shape of the temperature evolution. An example of a response of a jump into a non-transition region is plotted as a reference. In the upper panel the original signals (left) and the normalized (normalization of amplitudes) signals (right) are shown. In the lower panel the signal without the perturbation signal is drawn ( without (left) and with (right) normalization).

When plotting the normalized original temperature changes against each other one realizes that the relaxation processes must differ. The perturbation signal is the fastest process, whereas the signal gained at $T=298.076 \mathrm{~K}$ shows the slowest decay. This is more evident when plotting the normalized lipid responses. Therefore, for the quantitative analysis of the thermocouple signal just the signal after subtraction of the perturbation signal is used.

As shown later (see ch. 4) this method allows a time resolution of less than $500 \mathrm{~ms}$ which is about a factor of $6-8$ better than the time resolution of method I.

\subsection{Fluorescence Correlation Spectroscopy}

Fluorescence Correlation Spectroscopy (FCS) is a method which uses fluctuations in fluorescence intensity to analyze time dependent processes (Magde et al., 1972; Elson and Magde, 1974; Magde et al., 1974). It can be used for the study of translational diffusion (Magde et al., 1972; Aragón and Pecora, 1976; Rigler and Mets, 1992; Rigler et al., 1993; Schwille et al., 1997; Korlach et al., 1999; Schwille et al., 1999; Hac et al., 2005), rotational diffusion (Ehrenberg and Rigler, 1974; Aragón and Pecora, 1976) or chemical kinetic rate constants 
(Magde et al., 1972, 1974).

In this thesis simulations of lateral diffusion processes are evaluated with FCS and related to experimental studies which were performed by A. Hac (Hac, 2004). In the following two sections FCS is mainly explained in respect to lateral diffusion and the setup is explained.

\subsubsection{Mathematical Foundations}

In this part an expression of the autocorrelation of fluorescence intensity fluctuations is to be derived. The main focus is on diffusion processes. At first the case of normal diffusion of one particle is considered.

The intensity of the fluorescence signal $F(t)$ of fluorescence labels in a defined confocal volume changes due to translational or rotational diffusion, chemical reactions or photophysical effects. The signal is evaluated through an autocorrelation of the signal:

$$
G(\tau)=\frac{\langle F(t) F(t+\tau)\rangle}{\langle F(t)\rangle^{2}}=1+\frac{\langle\delta F(t) \delta F(t+\tau)\rangle}{\langle F(t)\rangle^{2}},
$$

with $\delta F(t)=F(t)-\langle F(t)\rangle$ is the fluctuation of the signal at a given time $t$ and $\langle F(t)\rangle$ is the average intensity signal. Eq. 2.17 shows that it is physically seen equivalent to correlate the signal itself or its fluctuations, since the difference is just an additive factor of +1 . Therefore, the following derivations are based on the correlation of the fluctuations $\delta F(t)$ :

$$
G_{\text {fluct }}(\tau)=G(\tau)-1=\frac{\langle\delta F(t) \delta F(t+\tau)\rangle}{\langle F(t)\rangle^{2}} .
$$

Assuming the presence of just one fluorescence species in the confocal volume, one can write the fluorescence signal as:

$$
F(t)=\kappa \cdot Q \int_{V} C E F(\vec{r}) \cdot I(\vec{r}) \cdot C(\vec{r}, t) d V,
$$

where $\kappa$ is a constant which depends on the efficiency of the setup, $Q$ is the product of the absorptivity and fluorescence quantum efficiency, $C E F(\vec{r})$ (collection efficiency function) reflects the probability to detect a signal and depends on the actual setup, e.g. the pinhole size (Rigler et al., 1993). $I(\vec{r})$ represents the laser intensity and $C(\vec{r}, t)$ the label concentration and $d V$ is the volume element.

Looking at the fluctuations in concentration and using $I_{e m}(\vec{r})=I(\vec{r}) \cdot C E F(\vec{r})$ one obtains an expression for the fluctuations in the fluorescence signal $\delta F(t)$ :

$$
\delta F(t)=\kappa \cdot Q \int_{V} I_{e m}(\vec{r}) \cdot \delta C(\vec{r}, t) d V .
$$

The autocorrelation of eq. 2.20 can be written as:

$$
\begin{aligned}
G_{\text {fluct }}(\tau) & =\frac{\langle\delta F(t) \delta F(t+\tau)\rangle}{\langle F(t)\rangle^{2}} \\
& =\frac{\int_{V} \int_{V^{\prime}} I_{e m}(\vec{r}) I_{e m}\left(\vec{r}^{\prime}\right)\left\langle\delta C(\vec{r}, t) \delta C\left(\vec{r}^{\prime}, t+\tau\right)\right\rangle d V d V^{\prime}}{\left(\int_{V} I_{e m}(\vec{r})\langle C(\vec{r}, t)\rangle d V\right)^{2}}
\end{aligned}
$$


If the concentration fluctuations are a result of isotropic diffusion processes ${ }^{5}$ they can be described by Fick's second law. Then one can find an expression for the concentration correlation $\Phi\left(\vec{r}, \vec{r}^{\prime}, \tau\right)$ (Elson and Magde, 1974):

$$
\Phi\left(\vec{r}, \vec{r}^{\prime}, \tau\right)=\left\langle\delta C(\vec{r}, t) \delta C\left(\vec{r}^{\prime}, t+\tau\right)\right\rangle=\frac{\langle C\rangle}{(4 \cdot \pi \cdot D \cdot \tau)^{\frac{3}{2}}} \cdot e^{-\frac{\left(\vec{r}-\vec{r}^{\prime}\right)^{2}}{4 \cdot D \cdot \tau}},
$$

with $\langle C\rangle$ is the mean concentration of fluorescence labels in the confocal volume.

In Fluorescence Correlation Spectroscopy one usually assumes a Gaussian laser beam profile (Aragón and Pecora, 1976):

$$
I(\vec{r})=I_{0} \cdot e^{-2 \cdot \frac{x^{2}+y^{2}}{r_{0}^{2}}} \cdot e^{-2 \cdot \frac{z^{2}}{z_{0}^{2}}},
$$

where $I_{0}$ is the maximum laser intensity at the origin of the laser beam and $r_{0}$ and $z_{0}$ are the lateral and axial distances at which the laser intensity is decreased to $e^{-2}$. These distances allow us to define the confocal volume in which the fluorescence intensity fluctuations are monitored $^{6}$ :

$$
V_{\text {conf }}=\pi^{3} r_{0}^{2} z_{0}
$$

Analysis of eq. 2.21 using eqs. 2.22 and 2.23 results in (Aragón and Pecora, 1976; Rigler et al., 1993):

$$
G_{\text {fluct }}^{3 D}(\tau)=\frac{1}{V_{\text {conf }} \cdot\langle C\rangle}\left(\frac{1}{1+\frac{\tau}{\tau_{d}}}\right) \cdot\left(\frac{1}{\sqrt{1+\frac{r_{0}^{2} \tau}{z_{0}^{2} \tau_{d}}}}\right),
$$

with

$$
\tau_{d}=\frac{r_{0}^{2}}{4 \cdot D}
$$

$\tau_{d}$ is the average diffusion time of a diffusing label and $\langle N\rangle=V_{\text {conf }} \cdot\langle C\rangle$ is the mean number of labels in the confocal volume. This means that if $r_{0}$ and $z_{0}$ are known one automatically gains the diffusion constant of the diffusing label with autocorrelating the fluorescence signal or its fluctuations:

$$
G_{\text {fluct }}^{3 D}(\tau)=\frac{1}{\langle N\rangle}\left(\frac{1}{1+\frac{\tau}{\tau_{d}}}\right) \cdot\left(\frac{1}{\sqrt{1+\frac{r_{0}^{2} \tau}{z_{0}^{2} \tau_{d}}}}\right) .
$$

In the case of lateral diffusion the third term in eq. 2.27 can be neglected:

$$
G_{\text {fluct }}^{2 D}(\tau)=\frac{1}{\langle N\rangle}\left(\frac{1}{1+\frac{\tau}{\tau_{d}}}\right) .
$$

\footnotetext{
${ }^{5}$ In this project FCS is used for the study of isotropic diffusion processes in lipid membranes.

${ }^{6}$ The exact values of $r_{0}$ and $z_{0}$ and therefore of the confocal volume depend on the experimental setup.
} 
So far just the case of normal diffusion has been discussed. Often in the case of diffusion in cell membranes one talks about anomalous diffusion in which the MSD is not linear with time, but is linear to $t^{\alpha}(0<\alpha \leq 1)$. Schwille and collaborators have introduced an expression for the autocorrelation function in the case of anomalous diffusion (Schwille et al., 1999):

$$
G_{\text {fluct }}^{\text {ano } 2 D}(\tau)=\frac{1}{\langle N\rangle}\left(\frac{1}{1+\frac{\Gamma \tau^{\alpha}}{r_{0}^{2}}}\right),
$$

where $\Gamma$ is the transportation coefficient.

The introduced result of the autocorrelation function can also be modified in the case that there are different diffusing species which means that there are labels with different diffusion constants. Korlach et al. (1999) have introduced an expression for two diffusing species which, however, can easily been generalized:

$$
G_{m c o m p}^{2 D}=\frac{1}{\langle N\rangle} \sum_{i} \frac{r_{i}}{1+\frac{1}{\tau_{d, i}}},
$$

with $\tau_{d, i}$ the mean diffusion time of species $i$ and $r_{i}$ the respective ratios.

\subsubsection{Experimental Setup}

FCS uses fluctuations in the fluorescence signal to get information about e.g. chemical rate constants or diffusion processes. It is necessary to define a small confocal volume as it is realized through a confocal setup which is schematically shown in fig. 2.9. Our FCS setup was built by A. Hac and a detailed description of the used setup can be found in Hac (2004).

A Nd:Yag laser from Crysta Laser (Reno/NV, USA) with an emission maximum at a wavelength of $532 \mathrm{~nm}$ (green) emits coherent, linearly polarized light. The laser light is reflected through a dichroic mirror into a water immersion objective (Olympus, Hamburg, Germany). Fluorescence markers which are stimulated by the laser light emit fluorescence light of another wavelength than the laser light wave length. The wave length of the fluorescence light is chosen so that the dichroic mirror is transmittant. It passes through a pinhole which is crucial in defining the detected confocal volume of the setup. Changing its size results in a bigger or smaller detection volume. Two avalanche photodiodes (Perkin Elmer, Boston/MA, USA) monitor the fluorescence signals of the parallel or the perpendicular polarized light. These signals are correlated by a hardware correlator card from Correlator.com (Bridgewater/NY, USA).

In fig. 2.10 results from example FCS measurements are shown. They directly show the principle of our experiments and simulations. Three examples in which the diffusion constants differ are shown. Rhodamine $6 \mathrm{G}$ is a small fluorescent molecule. TRITC is a lipid analog with fluorescence properties. The latter one can be used for diffusion studies in lipid membranes. Diffusion of TRITC in a lipid membrane is slower than diffusion of Rhodamine $6 \mathrm{G}$ in water. Therefore, the fluctuations in fluorescence intensity of Rhodamine $6 \mathrm{G}$ are faster. The autocorrelation profile decreases at shorter times since the mean diffusion time of Rhodamine $6 \mathrm{G}$ is smaller than the one of TRITC. Since the diffusion of TRITC markers in the "ld" phase is faster than in the "so" phase, the fluctuations in the first case are also faster than the ones of the second case. This is also reflected by the autocorrelation profiles. The 


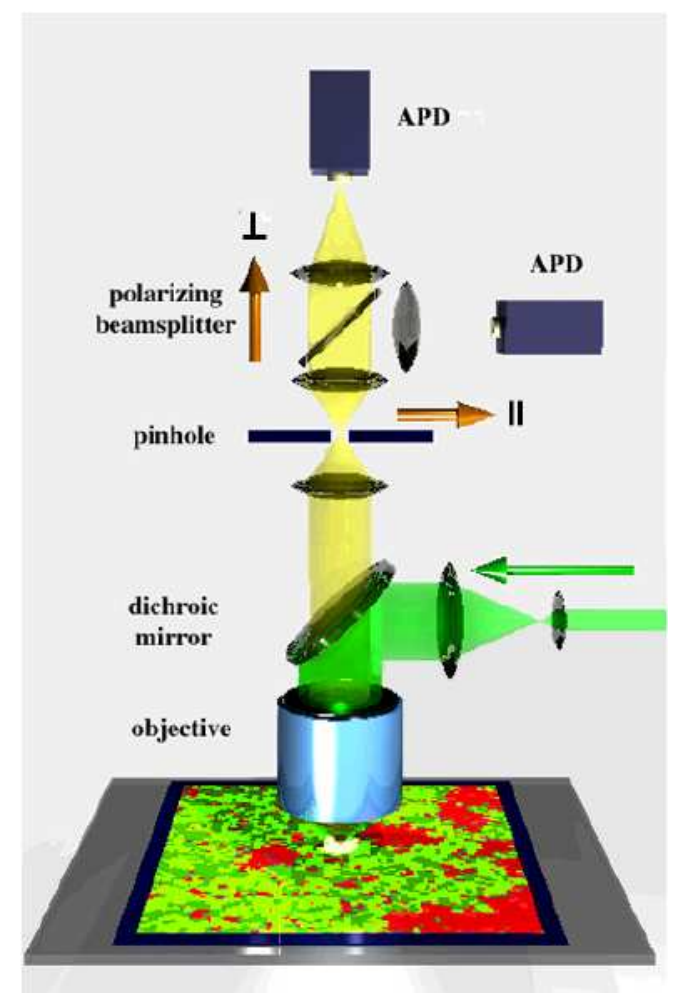

Figure 2.9: Scheme of a FCS setup. Laser light is reflected by a dichroic mirror into the microscope objective. In the sample the laser light stimulates fluorescence labels. These ones emit light of another wavelength. This travels through the dichroic mirror and a pinhole. Depending on polarization the photons are monitored by one or the other avalanche photodiode.

faster fluctuations result in a decay at short times. The mean diffusion time is shorter. Using eq. 2.27 for diffusion in $3 \mathrm{D}$ or using eq. 2.28 in $2 \mathrm{D}$ the autocorrelation curves allow a quantitative evaluation of the diffusion processes.

\subsection{Sample Preparation}

In this thesis results of studies on artificial phospholipid membranes are presented. Differential scanning and pressure perturbation calorimetry experiments were carried out by the author of this thesis. Experimental diffusion measurements were performed by A. Hac (Hac, 2004).

For the calorimetric experiments multilamellar and large unilamellar lipid vesicles were used. Diffusion measurements were done on stacks of supported lipid bilayers. Here just the preparation of the calorimetric samples is described. The preparation of the supported samples for FCS measurements can be found in the dissertation of A.Hac (Hac, 2004).

Lipids were purchased from Avanti Polar Lipids (Birmingham/AL, USA) and used without further purification. All lipid samples were dissolved in a $10 \mathrm{mM}$ Hepes buffer with $1 \mathrm{mM}$ EDTA and $\mathrm{pH}$ 7. The $\mathrm{pH}$ of the buffer was adjusted through adding natriumhydroxid to a millipore water, $10 \mathrm{mM}$ Hepes, $1 \mathrm{mM}$ EDTA solution. Where Hepes buffers between $\mathrm{pH} 6$ and 8, EDTA binds calcium which shall prevent the growth of bacteria. 

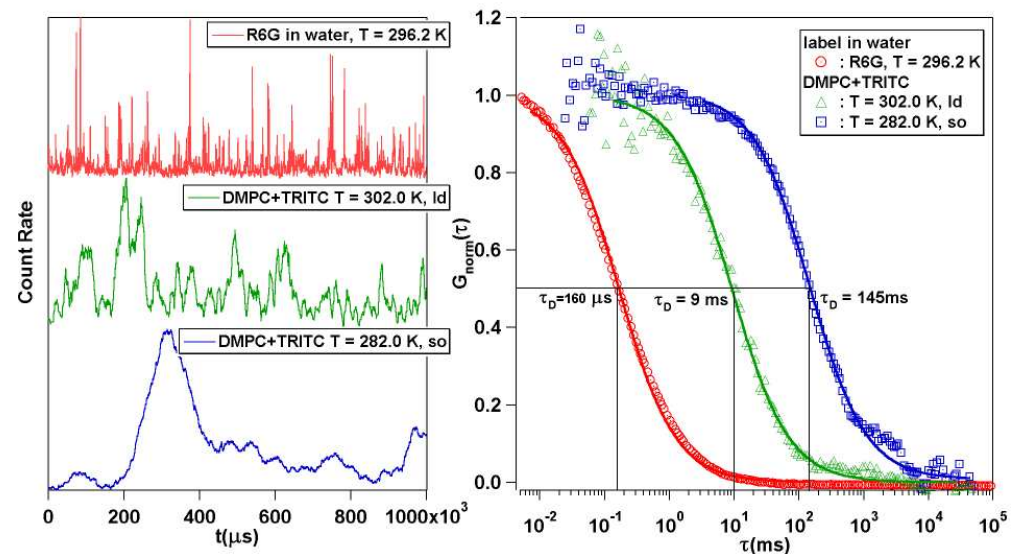

Figure 2.10: Example fluorescence intensity traces and their autocorrelation profiles. (left) Fluorescence intensity traces of fluorescence labels with different diffusing properties. In this example the small fluorescent molecule Rhodamine $6 \mathrm{G}$ diffuses in water. Its diffusion is faster than the diffusion of the TRITC labels in a lipid membrane in "ld" or "so" phase. The diffusion of the TRITC labels in the "ld" lipid membrane are faster than in the "so" lipid membranes. The different diffusion behavior can already be seen in the fluctuations and the speed of the changes. (right) If the diffusion of a particle is faster its mean diffusion time through the confocal volume is lower and the autocorrelation profile decreases at shorter times than if the diffusion is slower.

The measurements were done on multilamellar (MLVs) or large unilamellar vesicles (LUVs; diameter of about $100 \mathrm{~nm}$ ), meaning the vesicles originated from one bilayer formed to a vesicle (LUV) or a stack of bilayers lay on each other and formed a vesicle (MLV).

Pure multilamellar lipid vesicles were prepared through adding the buffer to the dry lipid powder and stirring it above the melting transition midpoint for $1 h$. During the stirring process the suspensions were vortexed $3-4$ times.

For the preparation of unilamellar lipid samples at first suspensions of multilamellar lipid vesicles (MLV) were prepared. Then these MLV suspensions were extruded with an Avestin extruder (Avestin Europe GmbH, Mannheim, Germany). The extruder exists of a system of two syringes which are connected with each other through a special filter system. The whole can be placed in a metal block. It is temperature controlled with a heat bath. Before filling the syringes of the extruder setup with the lipid solution they were kept in the heated extruder block $(T=320.9 \mathrm{~K})$ for 15 minutes. After that $1 \mathrm{ml}$ of the lipid solution was taken into one of the two syringes. Next the syringes were put back into the extruder block and the extrusion process was not started for another 15 minutes. At last the extrusion process started and the lipid sample was pressed through polycarbonate membranes with a pore diameter of $100 \mathrm{~nm}$ for one hour. During the extrusion each of the syringes was emptied approx. 60 times. At the end of the extrusion process the LUV solution was used which remained in the syringe which was not filled at the beginning of the extrusion process. Taking the sample from this syringe ensures that all lipid vesicles went through the polymer filter at least once.

Samples of two component lipid vesicles were prepared through dissolving each of the lipid components separately in organic solvent (dichloromethane : methanol 2:1). Then the two components were mixed. This mixture was dried through heating and a nitrogen or air 
stream. The remaining organic solvent was removed through desiccating the sample in a high vacuum desiccator over night. The further preparation is equivalent to the already described preparation of multilamellar vesicles.

In some of the measurements anesthetics, neurotransmitters or peptides were added. As anesthetics octanol (1-octanol; Sigma-Aldrich, St. Luis/MO, USA) and halothane (SigmaAldrich Inc., St. Luis/MO, USA) were used. Further on the neurotransmitter serotonin (Hydrochloride; Sigman-Aldrich Inc., St. Luis/MO, USA) or the peptide gramicidin A (Fluka, Buchs, Switzerland) were added to the lipid vesicles.

Concerning the preparation of the lipid/anesthetics samples, anesthetics were added to an already prepared buffer/lipid sample solution. This solution was stirred above the melting transition again for $30 \mathrm{~min}$ before it was put into the fridge for $15 \mathrm{~min}$. Then the same procedure was repeated at least two times. This was done because the permeability of a lipid membrane increases in the phase transition regime (Papahadjopoulos et al., 1973; Cruzeiro-Hansson and Mouritsen, 1988; Makarov, 2005), which was thought to help an incorporation of the alcohol molecules. Through heating and cooling with the choice of appropriate temperatures one automatically goes through the transition regime. However, the partition coefficient of alcohols also depends on the lateral structure of the lipid membrane. It displays a maximum in the melting transition (Trandum et al., 1999). It might be that the process of repeated heating and cooling gives no advantages but it is definitely not disadvantageous.

In the case of lipid/serotonin mixtures serotonin was added to the buffer solution and the whole stirred for 30min. Then, the lipid powder was dissolved in the buffer/serotonin solution. The rest of the preparation procedure equals the one of the preparation of MLVs. During the preparation the sample was also cooled below the melting transition midpoint and heated again at least two times. The lipid membrane goes through the phase transition regime when it is cooled below and heated above the melting temperature. The permeability increases in the phase transition regime. Therefore, it is believed that the serotonin molecules incorporate better into the multilamellar vesicles going through the phase transition regime.

The preparation of lipid/gramicidin A systems equals the one of the preparation of two component lipid mixtures. The dry lipid powder and the dry gramicidin A were separately dissolved in organic solvent (dichloromethane:methanol 2:1). Then both solutions were mixed. The sample was dried through heating and the application of a nitrogen or air stream. Over night the sample was kept in a high vacuum desiccator. After that the buffer was added and the sample was stirred above the transition midpoint temperature for $1 h$. Additionally the sample was vortexed $3-4$ times.

Samples which were used for DSC measurements and pressure jump experiments were degassed for at least $15 \mathrm{~min}$.

\subsection{The Chip Calorimeter}

The main focus of this thesis is the study of domains formation processes in artificial membrane systems. This study is also aimed at gaining a deeper understanding of processes in biological membranes. Domain formation processes in biomembranes are estimated to happen in the $100 \mathrm{~ms}$ regime or even faster. Therefore, relaxation time measurements on biological membranes cannot be investigated with the two previously described methods. Therefore, it seems worthwhile to think about new strategies. One possibility is the usage of a commercially available chip (NCM-9928, Xensor Integration, Delft, Netherlands). It is assumed 
that this chip can recognize heat transfer processes with a time resolution below $100 \mathrm{~ms}$ (Caspary et al., 1999). Chips of this kind are already successfully in usage as scanning, batch and flow-through calorimeters (Lerchner et al., 1998). In general also these techniques are of use in our lab, so that the constructed calorimeter shall fulfill the following criteria:

- possible to use as a differential scanning calorimeter,

- usage as a titration calorimeter,

- allowing the study of relaxation processes,

- possibility to extend it for flow-through applications.

Up to now mainly the construction of the mechanical parts is finished. Electronic equipment still needs to be built. Therefore, no data can be presented, but the principal construction is explained. Further steps and suggestions for possible realizations are mentioned, too.
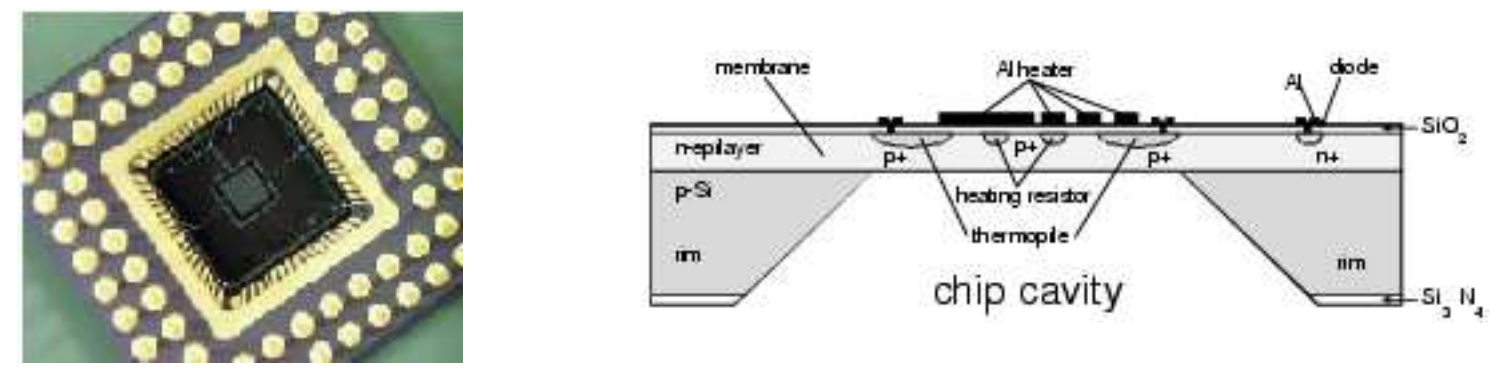

Figure 2.11: NCM-9924 chip. (left) A back view of the chip is shown. One sees the PGA and its 68 gold pins. One can recognize the silicon layer, the integrated thermopiles and therewith the chip's active area. (right) A schematic drawing of the chip is displayed. Thermopiles are incorporated into a silicon layer. A heater is implemented into the layer as well. Two additional aluminum heaters lie on top of the silicon membrane.

The chip calorimeter is based on the chip NCM-9928 (see fig. 2.11) from Xensor Integration (Delft, Netherlands). The chip consists of silicon-aluminum thermocouples which are connected in series and which are integrated into a silicon epitaxy layer. These thermocouples define the sensor of the chip. The whole silicon membrane is encapsulated in a ceramic PinGrid-Array (PGA) with 68 gold pins of size $68 \mathrm{~mm} \times 68 \mathrm{~mm}$. The chip itself sits in the PGA and has a dimension of $10 \mathrm{~mm} \times 10 \mathrm{~mm}$ with a membrane size of $8.3 \mathrm{~mm} \times 8.3 \mathrm{~mm}$. The thickness of this membrane is $45 \mu \mathrm{m}$. The thermocouples measure temperature differences between the active site and the rim of the silicon layer with a sensitivity of $50 \frac{\mathrm{mV}}{\mathrm{K}}$. Integrated into the silicon layer is a resistor heater with $800 \Omega$. Two aluminum heater $(1 \mathrm{k} \Omega$ and $250 \Omega)$ are brought onto the silicon layer and are galvanically isolated from the thermopiles. In the realization of our calorimeter two chips are used.

The principal construction of the calorimeter is based on three parts. It is an isolation, an outer calorimeter and an inner calorimeter shell. Each of these parts can be opened. The isolation shell separates the calorimeter shells and the outer environment. It is constructed from aluminum. Isolation material shall be brought between the isolation shell and the outer calorimeter shell, too. The inner and outer calorimeter shells are the parts which realize the function of the calorimeter. The outer calorimeter shell acts as a heat bath, whereas the chips 


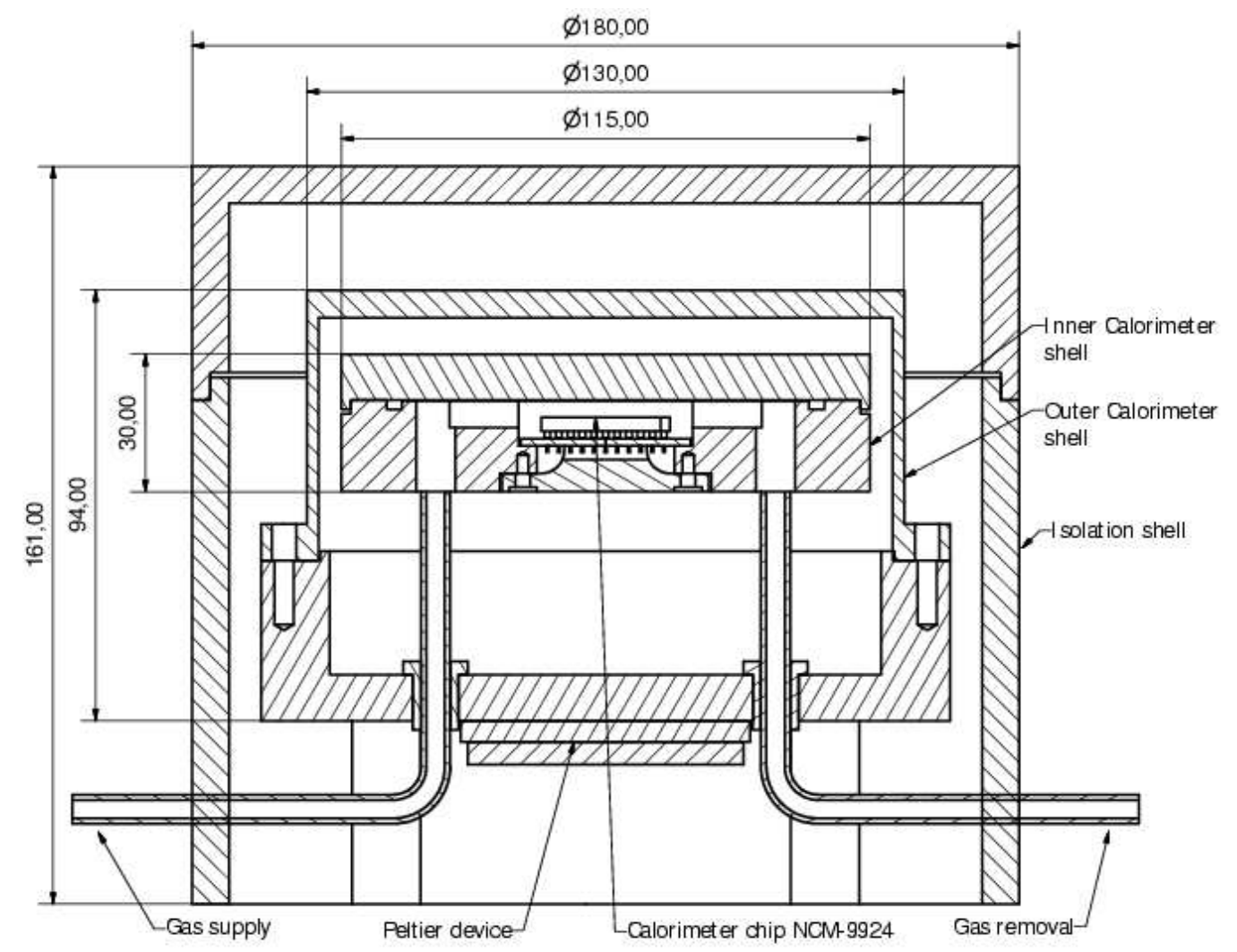

Figure 2.12: Dimensions of the chip calorimeter. The chip calorimeter consists of an isolation, an outer calorimeter and an inner calorimeter shell. All given values are in units of $\mathrm{mm}$.

can be found in the inner shell. The dimensions of the shells can be seen in fig. 2.12. Two tubes are added so that the pressure of the inner calorimeter shell can be varied.

The outer calorimeter shell is mounted on ceramic feet and is built out of brass. On its lower part a peltier device (Telemeter Electronic, Donauwörth, Germany) is attached. It adjusts the outer shells temperature which is measured with a PT 100 (platinum resistor, $100 \Omega$ at $0{ }^{\circ} C$, Farnell, Denmark). The inner calorimeter shell sits in the outer shell and it is held through the two pressure tubes. To minimize heat conduction to or from the inner shell, ceramic contacts are attached. Still, heat conduction might be present. The inner calorimeter shells temperature is controlled through the outer shells temperature which acts as a heat bath. For fine adjustments a $10 \mathrm{~W}$ power heater is mounted on the shell. The inner calorimeter shell is mainly constructed out of brass. On the bottom, however, a glass epoxy plate with copper coating is mounted. It is glued onto the bottom by a conductive epoxy glue (ITW Chemtronics, Kennesay/GE, USA). The glass epoxy plate has a hight of $1.6 \mathrm{~mm}$ with a copper coating of $50 \mu \mathrm{m}$ on each side. In it two grid sockets with 68 pins each are installed. They allow the mounting of the two chips and electrical contact with the control electronics. Between the two sockets a PT 100 with $100 \Omega$ at $0{ }^{\circ} C$ (Farnell, Denmark) is placed for measuring the inner calorimeter shells temperature. On the bottom the glass epoxy plate is supported by another brass block. 

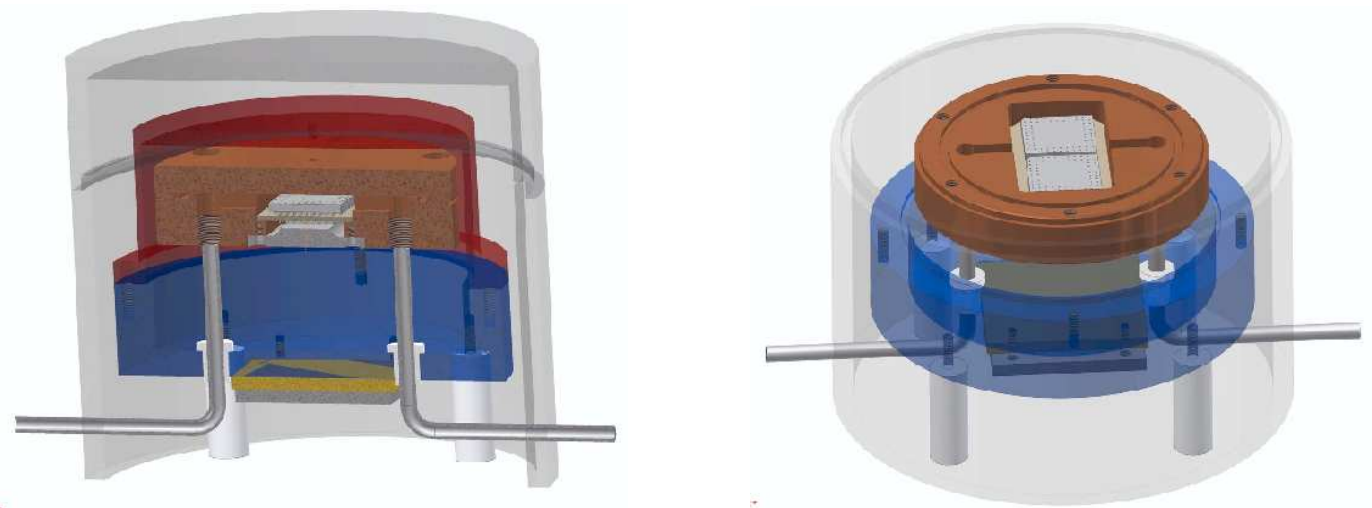

Figure 2.13: Chip calorimeter perspective views. A cross-sectional view (left) and a top view (right) of the calorimeter are drawn. It is composed of the isolation shell (transparent), the outer calorimeter shell (red and blue) and the inner calorimeter shell (brown). Tubes (grey) allow the adjustment of the inner pressure. A peltier device (yellow) is attached to the outer calorimeter shell and allows the adjustment of the outer shells temperature. On the outside of the inner shell a power heater is mounted. It is not shown in the drawing. The two calorimeter chips are sticked onto the two grid sockets (grey).

In fig. 2.13 two perspective views of the calorimeter are given. One shows the cross section (left) and the other one a top view (right) of the calorimeter. The transparent part is the isolation shell, the red and blue parts are the upper and lower parts of the outer calorimeter shell. The brown part is the inner calorimeter shell with the glass epoxy plate and the grid sockets (grey). The gas tubes, its isolation (white) and the peltier device (yellow) can also be easily seen. Not shown is that the lids of the inner and outer shell have holes which allow the insertion of two syringe needles. These holes can be closed.

The control of the calorimeter shall be realized with a self-written Labview (National Instruments, Austin/TX, USA) program. When finished it controls a PCI A/D card (PCI AD 16 DAC4, Kolter Electronics, Erftstadt, Germany), a Keithley GPIB card PCI-488 (Keithley Instruments Inc., Cleveland/OH, USA) and the printer port of the control PC. The latter one is used for the control of two coaxial solenoid valves (Nova Swiss, Effretikon, Switzerland) which allow pressure increase or decrease through addition or release of nitrogen gas. The GPIB card is needed for the control of a Keithley 2001 multimeter (Keithley Instruments Inc., Cleveland/OH, USA) and the belonging nanovolt amplifier Nanovolt Preamp Model 1801 (Keithley Instruments Inc., Cleveland/OH, USA). It reads in the voltage signals of the difference signal of the two NCM chips. The PCI A/D card is used for the regulation of the peltier device's current and voltage, the current through the power heater, the control of heat signals of the chip's heaters and the read out of the outer and inner calorimeter shells temperatures. The two temperatures are needed as a feedback mode of the current and voltage of the peltier device and power heater. It is chosen to control the peltier device and the power heater currents and voltages purely with the Labview program. Additional electronic devices are not used. The inner calorimeter's temperature of course gives also the sample's temperature. The chip heaters are useful for the calibration of the calorimeter chips. They also find application in calibrating the setup before each experiment. They can also perturb the system through inducing a short heat pulse. 
Currents and voltages of the peltier device and the power heater are adjusted with a selfbuilt device using power transistor technology. Signals from the PT-100 thermometers are amplified with a self-constructed device.

So far the calorimeter has not been used for any experiments. It is still under construction, meaning that details of the construction still need to be adjusted. The principal construction, however, is currently not under discussion. In the following paragraphs some remarks about possible applications and problems shall be given.

As mentioned in the beginning of this section the calorimeter can in principal be used for differential scanning calorimetry, titration calorimetry, perturbation calorimetry and flowthrough calorimetry.

Since the used sample substances are small in volume evaporation might occur which affects the signal. To avoid this it is planed to add some sponges which are soaked with buffer solution. This should lead to a constant vapor pressure and should avoid evaporation of the sample.

For all techniques the characteristics of the chips need to be known like the thermopiles sensitivity as a function of temperature. These can be determined using the chip heaters.

Using it as a differential scanning calorimeter should be realized without encountering big problems. Of course, an exact temperature control with a constant scan rate has to be established.

More problematic is using it as a titration calorimeter. The temperature needs to be kept constant, which should be easily realized. Addition of titration liquid can be realized through a simple Hamilton syringe system in the beginning. In later stages more sophisticated software controlled techniques could be applied. Addition of the titration substance, however, might lead to an offset in the signal (Lerchner et al., 1999). As well, mechanical forces might perturb the calorimeter signal. These might occur because the liquid drop coming from the titration syringe touches the chip and exerts forces. This can be more pronounced using a pump system (personal communication J. Lerchner and Gert Wolf, University of Freiberg, Germany).

Using it for perturbation calorimetry one can follow two strategies. One is the addition or release of pressure. A second method is increasing the sample temperature for a short time through a heat pulse of one of the chip heaters.

The first method seems to lead to many problems. One problem is the mechanical stability of the chip membrane. Its size is $10 \mathrm{~mm} \times 10 \mathrm{~mm}$ at a thickness of $45 \mu \mathrm{m}$. After a pressure change the pressure builds up in the calorimeter cell. In an ideal experiment the pressure on both sides of the chip membrane is the same at all times. In reality, however, this must not be the case or else pressure gradients might destroy the chip. A support of the membrane is technically difficult to realize and might lead to a changed chip time resolution, resulting in a worse time resolution. This means that just small pressure changes can be done which might lead to a weak signal from the membrane system because the calorimetric profiles overlap and therefore the heat release or absorption is little. A second problem is the temperature stability in the inner calorimeter shell. Performing pressure release leads to cooling because of the expansion of the gas. Adding pressure requires that the gas is preheated since the gas temperature and the inner calorimeter temperature might be different. The compression also leads to a temperature increase.

At this stage of the planing it looks more promising to use one or more of the built-in chip heaters to induce a heat pulse. The sample temperature will increase, but then relax back to the inner calorimeter's temperature. An important question, of course, is the time scale of the temperature relaxation. This one might be a limit to the time resolution of the 
relaxation process. The method with changing temperature has the advantage that different temperature changes can be easily induced.

The last mode is to use the calorimeter as a flow-through calorimeter. These chips have already been used by these means (Lerchner et al., 1998). The principal construction should be easy using the chosen calorimeter layout. One application might be to perturb the membrane systems through a change in $\mathrm{pH}$ or ion concentration as done by Strehlow and Jähnig (1981). The difference would be, however, that relaxation processes can be studied by means of thermodynamics and not by fluorescence techniques, as done by Strehlow and Jähnig (1981).

It can be concluded that the introduced calorimeter might be a helpful tool in the study of different thermodynamical processes in artificial and biological membranes. Undoubtedly there are still a lot of design and construction problems that will arise and require solving before a successful chip calorimeter is realized. 


\section{Chapter 3}

\section{Theory}

\subsection{Modeling Lipid Membrane Systems}

Different kinds of models to describe the physical behavior of lipid membrane systems can be found in the literature. These range from analytical to numerical models. In the first part of this chapter a short review about models as they can be found in the literature is presented. In the second part a statistical mechanics model for the description of the phase behavior of a two component lipid system is explained in detail. This model is used for diffusion, relaxation and fluctuation studies of the modeled system. The last part introduces an analytical approach to explain the relaxation behavior of domain formation processes as suggested by Grabitz et al. (2002). The validity of the results of this analytical model is shown using the statistical physics model introduced in the second part of this chapter.

\subsubsection{Models in the Literature}

Since the 1970s phase transitions of bilayer membrane systems are theoretically described with the help of statistical physics. In the beginning different mean-field theories were presented, which have nowadays been overtaken by numerical methods like Monte Carlo simulations or Molecular Dynamics.

Nagle (1973) was the first one, who gave a statistical mechanical description of lipid bilayer melting phenomena by means of a cooperative change in the intrachain conformation distribution of the lipid chains. This is a very minimalistic model which can be exactly solved. It includes steric repulsions, rotational isomerization and attractive interactions. It is, however, not very close to reality.

In the same year Marcelja (1973) published a mean-field approach which was further elaborated in Marcelja (1974). It neglects steric interactions, but puts its emphasize on the internal energy of a single lipid and dispersive or van der Waals interactions. One result of this study is that the melting transition, while being first order in character, is rather close to a critical point. Doniach (1978) simplified the description further and was the first to introduce a twostate model for a better understanding of lipid bilayer melting transitions. Doniach (1978) used the model to relate lateral compressibility and fluctuations in bilayer density with bilayer permeability. Further on, Doniach concludes that the transition is weakly first order and close to a critical point. He points out that this might be important for the thermodynamical understanding of interactions in lipid mixtures with and without proteins. 
Marcelja (1976) was the first one to report a model about lipid-mediated protein interaction. It was an extension of his earlier work on lipid membranes without proteins. Interactions between lipids and proteins were modeled through the addition of a constant interaction parameter. For their research on protein-lipid interactions Pink and Chapman (1979) used a lattice model. Lipid chains were taken to be either in a ground or excited state as done by Doniach (1978). Nearest neighbor van der Waals and steric interactions were introduced and it was assumed that the polar group interactions of lipids can be represented by effective pressure-area terms. The interaction with the protein was modeled by adding additional interaction terms. Later the same authors published a model to describe experimental Raman scattering results (Pink et al., 1980). In the literature this model is known as the "Pink Model". Its main feature is that it assumes one all-trans, one melted and eight intermediate chain configurations. At the time of publication it was evaluated by means of mean-field theory. Pink's model, however, has become important in the numerical study of melting profiles using the Monte Carlo simulation technique. Sperotto and Mouritsen (1991) combined Pink's model with the microscopic interaction model as introduced in the already mentioned paper by Pink and Chapman (1979) and the mattress model (Mouritsen and Bloom, 1984) which is explained in section 1.1.3. Further, it was also successfully used for the description of two component lipid systems and their melting behavior (Mouritsen, 1990; Jørgensen et al., 1993; Jørgensen and Mouritsen, 1995). For the description of the melting profile of one and two component lipid systems (Sugar et al., 1994; Jerala et al., 1996; Sugar et al., 1999) and lipid/protein systems (Heimburg and Biltonen, 1996) a two-state model based on the ideas of Doniach (1978) is sufficient.

One advantage of numerical methods in comparison to mean-field approaches is that besides the evaluation of average thermodynamical parameters like heat capacity they allow the analysis of the distribution of lipids. The domain structure of these systems can be evaluated (Sugar et al., 2001; Hac et al., 2005; Seeger et al., 2005). Domain formation on different length scales can be found.

Due to increasing computer power the usage of Molecular Dynamics has become more popular (Feller, 2000). Atomistic models are used and Newton's equations have to be solved, which is very time consuming. Therefore, the time scale simulated using this technique has still not exceeded the 100 ns regime. Considering that many biological processes happen in the ms regime one has to be careful using this technique and especially with the interpretation of results obtained (see i.e. (Almeida et al., 2005)).

In this thesis the model by Sugar et al. (1999) is adopted and refined in order to accompany experimental work. This model is evaluated by numerical means (Monte Carlo simulation). It can describe the melting behavior of a two component lipid system (DMPC/DSPC). It is a lattice model considering just nearest neighbor interactions and assuming two different lipid chain states. Diffusion steps are included as well. A more detailed description is given in the following section.

\subsubsection{A Model for Describing the Phase Behavior of a DMPC:DSPC Sys- tem}

As mentioned in the previous section, a two state (Ising) model for the description of the melting of one component lipid membranes was introduced by Doniach (1978). This model was adopted by Sugar et al. (1994) and evaluated by means of Monte Carlo simulations. Later this model was further developed for the study of the phase behavior of a two component 
lipid system (Sugar et al., 1999).

Here, the model as introduced by Sugar et al. (1999) is used and is refined for studying diffusion $^{1}$, relaxation and fluctuation properties of a two component (DMPC:DSPC) lipid system. DMPC is a phospholipid whose two chains have 14 carbon atoms each. DSPC has longer chains which have 18 carbon atoms.

In the model each lipid is represented through its two chains. These are assumed to be either in an ordered or a disordered state. It is possible that the two chains of one lipid are in opposite states. The enthalpies and entropies of ordered and disordered chains vary and depend on the lipid species. The enthalpy and entropy differences between disordered and ordered chains are assigned the symbols $\Delta H_{i}$ and $\Delta S_{i}$, where $i=1,2$ denotes the two different lipid species ${ }^{2}$. Lipid chains are arranged on a hexagonal lattice, meaning that each chain has six nearest neighbors; the coordination number $z$ equals 6 . Periodic boundary conditions are assigned for the lipids at the lattice boundaries.

$N$ is the total number of chains, $N_{i}$ the number of chains of species $i, N_{i}^{m}$ is the number of chains of species $i=1,2$ in state ${ }^{3} m=o, d, N_{i j}^{m n}$ the total number of nearest neighbors of species $i$ and $j$ in states $m$ and $n$. One finds a connection between the total number of lipid chains and the number of given pairs of nearest neighbor chains. The same is true for the number of chains of certain state and species and the number of given pairs of nearest neighbor chains:

$$
\begin{aligned}
\frac{z}{2} N= & N_{11}^{o o}+N_{11}^{o d}+N_{11}^{d d}+N_{12}^{o o}+N_{12}^{o d}+N_{12}^{d o}+N_{12}^{d d} \\
& +N_{22}^{o o}+N_{22}^{o d}+N_{22}^{d d} \\
z N_{i}^{m}= & 2 \cdot N_{i i}^{m m}+N_{i i}^{m n}+N_{i j}^{m m}+N_{i j}^{m n},
\end{aligned}
$$

where $(i \neq j)$ and $(m \neq n)$. This expression is needed for an algebraic transformation of the system's Hamiltonian.

First, the Hamiltonian of the system needs to be derived. A lipid chain of species $i$ in state $m$ has an assigned energy of $E_{i}^{m}$. The degeneracy due to different localizations and orientations of the rotational isomers in the lipid chain is represented by $f_{i}^{m}$. Nearest neighbors interact with each other and therefore one needs to consider nearest neighbor interaction energies $E_{i j}^{m n}$ with the degeneracies $f_{i j}^{m n}$. For a certain matrix configuration $\mathbf{S}$ one finds for the energy $E(\mathbf{S})$ and the degeneracy $f(\mathbf{S})$ :

$$
E(\mathbf{S})=\sum_{i=1}^{2} \sum_{m=o}^{d} E_{i}^{m} N_{i}^{m}+\sum_{m=o}^{d} \sum_{n=o}^{d} \sum_{i=1}^{2} \sum_{j=1}^{2} E_{i j}^{m n} N_{i j}^{m n}
$$

and

$$
f(\mathbf{S})=\prod_{i=1}^{2} \prod_{m=o}^{d}\left(f_{i}^{m}\right)^{N_{i}^{m}} \cdot \prod_{m=o}^{d} \prod_{n=o}^{d} \prod_{i=1}^{2} \prod_{j=1}^{2}\left(f_{i j}^{m n}\right)^{N_{i j}^{m n}} .
$$

\footnotetext{
${ }^{1}$ Applying the model to the study of diffusion in two component lipid membranes has already been part of my master's thesis (Seeger, 2002). In the past years the model has been changed in some details and studies have been extended.

${ }^{2}$ Here $i=1$ refers to the DMPC and $i=2$ to the DSPC lipid chains

${ }^{3}$ It is chosen that $m=o$ denotes the ordered and $m=d$ the disordered chain state.
} 
The probability to find a certain configuration $\mathbf{S}$ is given by:

$$
p(\mathbf{S})=\frac{e^{-\frac{\chi(\mathbf{S})}{k_{B} T}}}{Q\left(N_{1}, N_{2}, T, V\right)},
$$

with $\chi(\mathbf{S})=E(\mathbf{S})-k_{B} T \ln f(\mathbf{S}), k_{B}$ the Boltzmann constant, $T$ the temperature and $Q\left(N_{1}, N_{2}, T, V\right)$ the partition function.

Using eqs. 3.1, 3.2, 3.3 and 3.4 one can derive an expression for $\chi(\mathbf{S})$ which is the sum of a configuration dependent and a configuration independent part:

$$
\chi(\mathbf{S})=\chi_{\text {const. }}(\mathbf{S})+\chi_{\text {conf. }}(\mathbf{S}),
$$

with

$$
\begin{aligned}
\chi_{\text {const. }}(\mathbf{S}) & =N_{1}\left\{\left(E_{1}^{o}-k T \ln f_{1}^{o}\right)+\frac{z}{2}\left(E_{11}^{o o}-k T \ln f_{11}^{o o}\right)\right\} \\
& +N_{2}\left\{\left(E_{2}^{o}-k T \ln f_{2}^{o}\right)+\frac{z}{2}\left(E_{22}^{o o}-k T \ln f_{22}^{o o}\right)\right\}
\end{aligned}
$$

and

$$
\begin{aligned}
\chi_{\text {conf. } .}(\mathbf{S}) & =N_{1}^{d}\left(\Delta H_{1}-T \Delta S_{1}\right)+N_{2}^{d}\left(\Delta H_{2}-\Delta S_{2}\right) \\
& +N_{11}^{o d} \omega_{11}^{o d}+N_{12}^{o o} \omega_{12}^{o o}+N_{12}^{o d} \omega_{12}^{o d} \\
& +N_{12}^{d d} \omega_{12}^{d d}+N_{12}^{d o} \omega_{12}^{d o}+N_{22}^{o d} \omega_{22}^{o d}
\end{aligned}
$$

where the following definitions are used:

$$
\begin{aligned}
\Delta H_{i} & =\left\{E_{i}^{d}+\frac{z}{2} E_{i i}^{d d}\right\}-\left\{E_{i}^{o}+\frac{z}{2} E_{i i}^{o o}\right\} \\
\Delta S_{i} & =k_{B} \ln f_{i}^{d}-k_{B} \ln f_{i}^{o}-\frac{z}{2} k_{B} \ln \left(\frac{f_{i i}^{o o}}{f_{i i}^{d d}}\right), \\
\omega_{i j}^{m n} & =\left[E_{i j}^{m n}-\frac{E_{i i}^{m m}+E_{j j}^{n n}}{2}\right]-k_{B} T \ln \frac{f_{i j}^{m n}}{\sqrt{f_{i i}^{m m} f_{j j}^{n n}}} .
\end{aligned}
$$

During the simulation only the free energy difference between two configurations is needed. Therefore, the configuration independent part does not play a role and is neglected. This means that in the case of the binary lipid mixture ten parameters have to be determined. These are the enthalpy and entropy changes for each lipid species and a total of six interaction parameters. As explained in the following section all interaction parameters can be obtained from heat capacity profiles measured by Differential Scanning Calorimetry (see sec. 2.1).

\subsubsection{Determination of Model Parameters}

As mentioned in the previous section there are ten parameters which need to be determined if one wants to describe a two component lipid system. All these parameters can be obtained from experiments.

The enthalpy and entropy changes of the single chains going from the ordered to the disordered state can be obtained from heat capacity profiles of the one component lipid systems. 


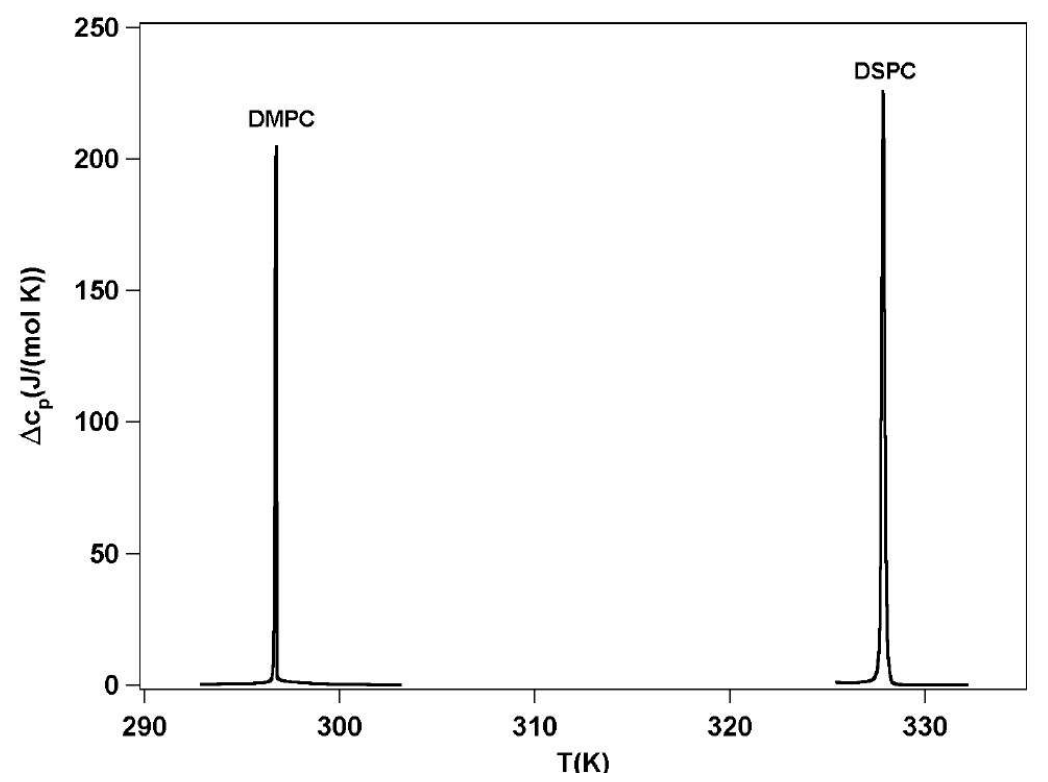

Figure 3.1: Excess heat capacity profiles of one component lipid systems. The shown excess heat capacities were measured on suspensions of multilamellar DMPC and DSPC lipid vesicles. They allow the determination of the enthalpy and entropy changes of each of the two melting transitions.

Fig. 3.1 shows the two excess heat capacity profiles of multilamellar DMPC and DSPC lipid suspensions as measured by DSC. The areas of the heat capacity curves define the enthalpy changes between an ordered and a disordered lipid of the respective species. At the transition midpoint, $T_{m}$, the Gibbs free energy is zero since the chemical potentials of the ordered and disordered phases are identical:

$$
\Delta G_{i}\left(T_{m, i}\right)=\Delta H_{i}-T_{m, i} \Delta S_{i}=0 .
$$

Knowing the enthalpy difference, $\Delta H_{i}$, and the transition midpoint, $T_{m, i}$, one can easily determine the entropy difference:

$$
\Delta S_{i}=\frac{\Delta H_{i}}{T_{m, i}}
$$

There are six remaining parameters which need to be found. These are the result of interactions between unlike nearest neighbors. They can be indirectly obtained from excess heat capacity profiles of two component lipid systems.

As described later in the text, the Monte Carlo simulation allows one to calculate the heat capacity profiles of DMPC:DSPC membranes of different composition (see sec. 3.1.5). The shape of the curves strongly depends on the choice of the interaction parameters. Comparing the results of simulations of various different DMPC:DSPC systems to measured heat capacity curves, including the one component systems, one can deduce all needed interaction parameters. The parameters used are shown in table 3.1. It is important to note that for the determination of these parameters several excess heat capacity profiles were used and they are constant for all compositions. 


\begin{tabular}{|c|c|}
\hline Parameter & $\frac{\mathrm{J}}{\text { mol.chain }}$ \\
\hline$\Delta H_{1}$ & 13165 \\
\hline$\Delta H_{2}$ & 25370 \\
\hline$\omega_{11}^{o d}$ & 1353 \\
\hline$\omega_{22}^{o d}$ & 1474 \\
\hline$\omega_{12}^{o o}$ & 607 \\
\hline$\omega_{12}^{d d}$ & 251 \\
\hline$\omega_{12}^{o d}$ & 1548 \\
\hline$\omega_{12}^{d o}$ & 1716 \\
\hline
\end{tabular} \mid \begin{tabular}{|c|c|}
\hline$\Delta S_{1}$ & 44.31 \\
\hline
\end{tabular}

Table 3.1: Parameter values used in the Monte Carlo simulations. They are slightly different from those used in Sugar et al. (1999). All numbers are given per lipid chain.

\subsubsection{Evaluation by Means of Monte Carlo Simulations}

In section 3.1.2 a statistical mechanical model for the description of a two component lipid system was introduced. This model is numerically evaluated by means of Monte Carlo simulations. A MC simulation performs a random walk through phase space. Every possible phase state is accepted with a certain probability. A MC simulation brings the system to its equilibrium and allows calculations of thermodynamical averages in the equilibrium state.

In order to perform Monte Carlo simulations it is essential to define Monte Carlo steps and Monte Carlo cycles. Here, two Monte Carlo steps are defined: state changes and diffusion steps. They are displayed in fig. 3.2. Lipid chains can be either in an ordered or disordered state. During the simulation changes from an ordered to a disordered state, or conversely, are allowed. There are two kinds of diffusion steps. One step is a possible rotation if the four involved chains form a rhombus ${ }^{4}$ (see fig. $3.2 \mathrm{~b}$ ). The other is a lateral movement of the lipid chains. In the case of a rotational diffusion step, only two lipid chains change their position. In the other case of lateral translation, the position of four chains is shifted. Concerning translational diffusion steps one has to add that during the simulation of the equilibrated system just neighboring lipids can be involved in a diffusion step. In the non-equilibrated system, when one tries to find the system's equilibrium, the exchange of the positions of two lipids which are not necessarily neighbors is also allowed. These non-physical diffusion steps help to hasten the finding of the system's equilibrium.

Changing the matrix configuration in the MC simulation is based on the MC steps, the swapping of lipid chain states or diffusion. A step can be accepted or not, which depends on the free energy difference of the old and new system configuration.

The exact procedure is the following:

- One starts from the actual system configuration $\mathbf{S}_{\mathbf{1}}$. A new configuration $\mathbf{S}_{\mathbf{2}}$ is generated.

\footnotetext{
${ }^{4}$ The lipid chains are arranged on a triangular lattice. A rotation of a lipid needs the exchange of two lipid chains. This is only possible if the four chains, when connected by imaginary lines, form a rhombus. Rotations of $\pm 60^{\circ}$ are possible.
} 

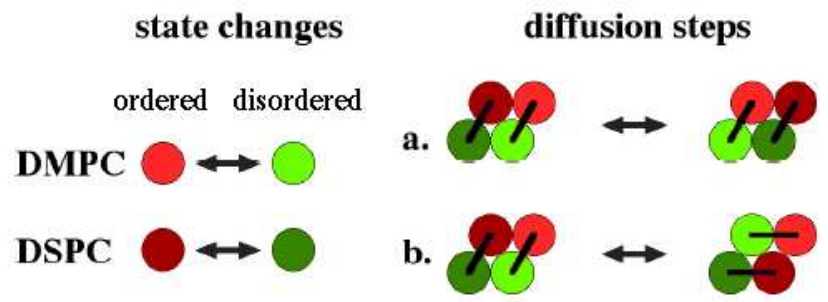

c.

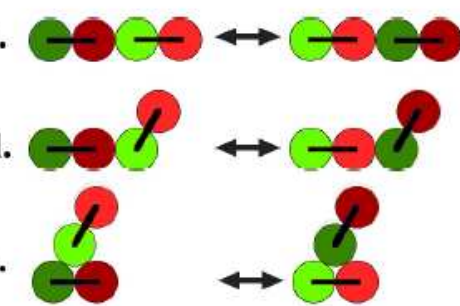

Figure 3.2: Lipid chain states and diffusion steps. (left) The lipid chain state can change from an ordered (red) to a disordered (green) configuration or vice versa. (right) Nearest neighbor lipids can undergo different diffusion steps. The bar represents the chemically linked lipid chains, which form one lipid. During the simulation this link has to be kept, since a lipid cannot exchange its chains.

- One calculates the free energy difference of the two configurations:

$$
\Delta G=\chi_{\text {conf }}\left(\mathbf{S}_{\mathbf{2}}\right)-\chi_{\text {conf }}\left(\mathbf{S}_{\mathbf{1}}\right)
$$

- The free energy difference allows one to calculate a Boltzmann factor:

$$
K=e^{-\Delta G /(R T)},
$$

with $R$ being the gas constant and $T$ the temperature.

- A probability $P=\frac{K}{1+K}$ is compared to a generated random number $\mathrm{RAN}^{5}$, with $0<$ $R A N<1$.

- If $R A N \leq P$ one accepts the new configuration $\mathbf{S}_{\mathbf{2}}$. If $R A N>P$ one discards the new configuration and keeps the old configuration $\mathbf{S}_{\mathbf{1}}$.

This is the so called Glauber algorithm (Glauber, 1963). The first time a Monte Carlo simulation was used the probability was set to $P=K$ or $P=1$, if $K \geq 1$. This is the Metropolis algorithm (Metropolis et al., 1953). Both algorithms lead to the same result.

With the introduced procedure and the Monte Carlo steps one can define Monte Carlo cycles. This defines order in which the MC steps are to be performed. Here, the Monte Carlo cycles are usually defined as follows: In the first $N$ steps, a random chain is chosen and it is asked whether this chain shall change its state. In the next $N$ steps two lipids are randomly picked out which might perform a lateral diffusion step. Then, in the last $N$ steps of the cycle, four chains are chosen which might do a rotational step. $N$ is the total number of lattice points which means $N / 2$ is the total number of lipids. In the cases that the Monte Carlo cycle is redefined it is mentioned in the text.

\footnotetext{
${ }^{5}$ In the simulation a pseudo random number is calculated after the method of L'Ecuyer (L'Ecuyer, 1988; Press et al., 1997). With one initialization the pseudo random numbers will start to repeat after about $2.3 \cdot 10^{18}$ pseudo random numbers.
} 


\subsubsection{Simulation of Heat Capacity Profiles}

Using the introduced model and evaluating it by means of Monte Carlo simulations one has the possibility of studying different properties. Sugar et al. (1999) were the first ones to evaluate a Doniach model for the description of the phase behavior of a two component lipid system by Monte Carlo simulations. They extended their studies to the understanding of cluster sizes and percolation thresholds of DMPC:DSPC lipid systems (Sugar et al., 2001; Michonova-Alexova and Sugar, 2002).

For the description of the phase behavior one analyzes heat capacity profiles. The heat capacity value $\left(\Delta c_{p}\right)$ of a defined DMPC:DSPC lipid system at a certain temperature is a direct consequence of macroscopic fluctuations in enthalpy:

$$
\Delta c_{p}=\frac{\left\langle H^{2}\right\rangle-\langle H\rangle^{2}}{N R T^{2}}
$$

with $H$ being the enthalpy, $\langle H\rangle$ the averaged enthalpy, $\left\langle H^{2}\right\rangle$ the averaged enthalpy squared, $N$ the total number of chains, $R$ the gas constant and $T$ the temperature. This is the fluctuation-dissipation theorem which can be derived from simple statistical physics relations (Hill, 1962) or from linear response theory (Kubo, 1966).
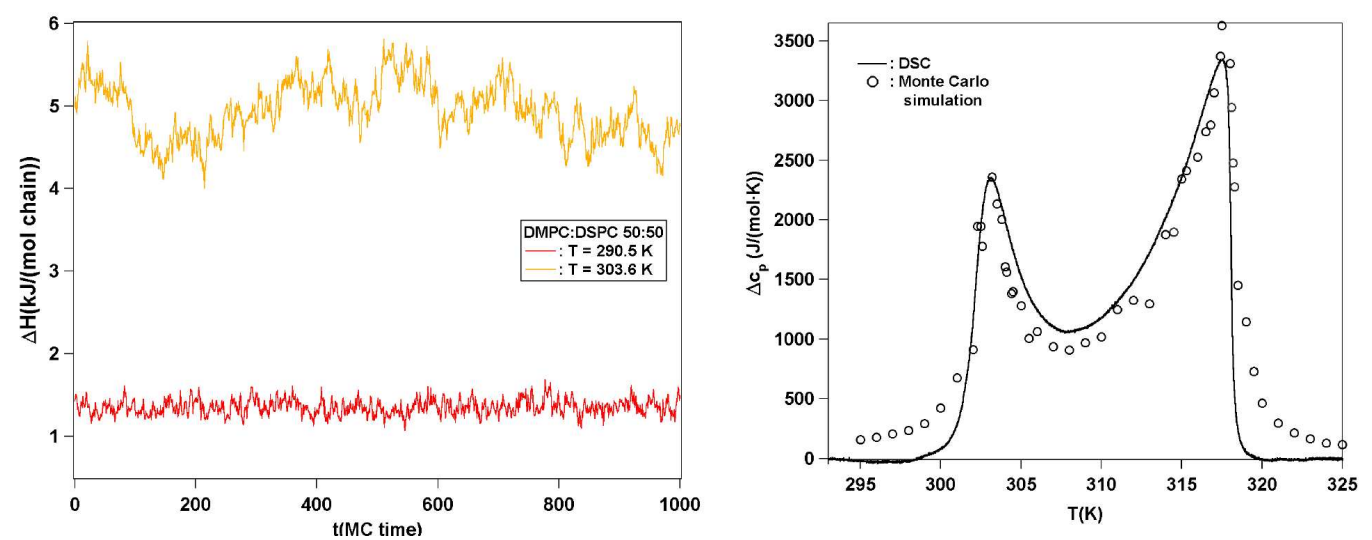

Figure 3.3: Enthalpy fluctuations and heat capacity. (left) Enthalpy traces from a simulation of an equimolar DMPC:DSPC lipid system at two different temperatures. (right) Simulated and measured excess heat capacity profiles of a DMPC:DSPC 50:50 lipid system.

As shown earlier (Seeger, 2002) the excess heat capacity values calculated using the fluctuation dissipation theorem agree well with the ones calculated from the derivative of the averaged enthalpy:

$$
\Delta c_{p}=\left(\frac{\partial\langle H\rangle}{\partial T}\right)_{p}
$$

In the left panel of fig. 3.3 enthalpy traces of a DMPC:DSPC 50:50 lipid system at two different temperatures are shown. It can be seen that the strength of the fluctuations depends on temperature. In the right panel an experimental excess heat capacity profile is fitted by a simulated one. Comparing the heat capacity results with the enthalpy traces, one finds that stronger fluctuations can be found at high excess heat capacity values. 


\subsubsection{Time Scales in Enthalpy Traces: Relaxation Time Studies}

One can use fluctuations in enthalpy to calculate the excess heat capacity value of the chosen lipid system at temperature $T$. The strength of the fluctuations, say the quadratic standard deviation, is proportional to the excess heat capacity. The enthalpy traces, however, contain more information. An autocorrelation ${ }^{6}$ profile of an enthalpy trace

$$
G(\tau)=\frac{\int_{0}^{\infty}(H(t)-\langle H\rangle)(H(t+\tau)-\langle H\rangle) d t}{\int_{0}^{\infty}(H(t)-\langle H\rangle)^{2} d t} .
$$

contains information about the time scales of the enthalpy fluctuations. This allows one to study the relaxation properties of the lipid system (see section 3.2).

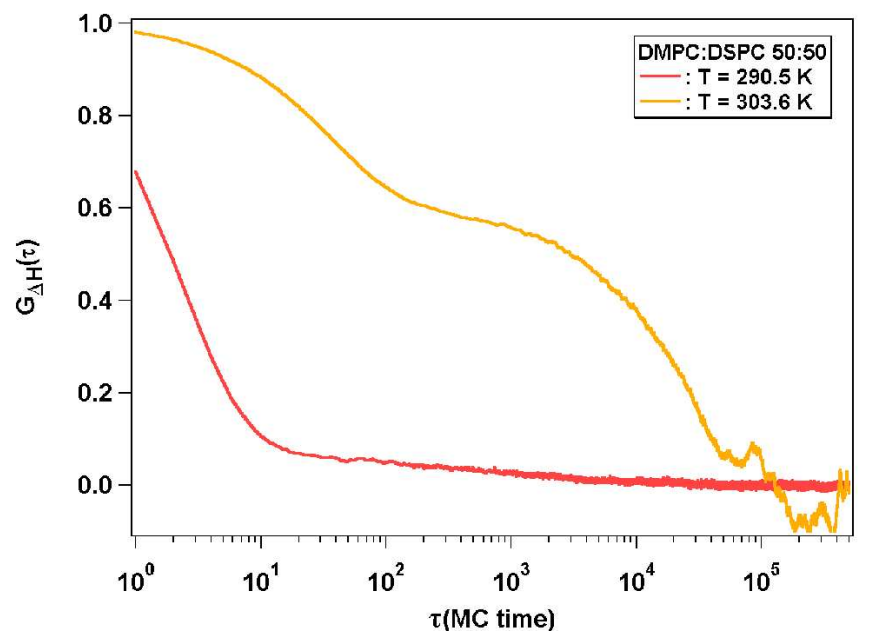

Figure 3.4: Autocorrelation curves of the enthalpy traces of fig. 3.3. One can see that the curves decay on different time scales and several relaxation processes might occur.

The autocorrelation profiles of the enthalpy traces of fig. 3.3 are displayed in fig. 3.4. The two curves decay at different times. The relaxation behavior can be governed by different time scales as can be clearly seen for the curve at $T=303.6 \mathrm{~K}$.

\subsubsection{Simulation of Fluorescence Correlation Spectroscopy Experiments}

Fluorescence Correlation Spectroscopy (FCS) allows one to measure diffusion properties of artificial and biological membrane systems (see section 2.3). One part of this thesis is based on studying diffusion properties in the two component lipid system of DMPC and DSPC. Experimental studies performed by A. Hac and described in Hac (2004) are accompanied by Monte Carlo simulation studies (this work) using the introduced two state model (see the previous sections).

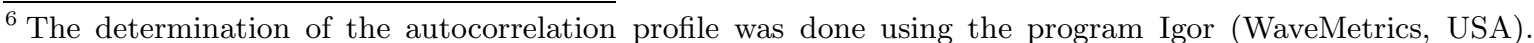
An autocorrelation is a convolution of the signal with itself. This can be expressed by means of Fourier transformations: $(f \otimes f)(\xi)=\mathcal{F}^{-1}\left[\mathcal{F}[f](\xi) \cdot \mathcal{F}[f]^{*}(\xi)\right]$, with $\mathcal{F}[f]$ being the Fourier transformation of function $f$. Igor provides a package for calculating an autocorrelation of a given vector using a Fast Fourier Transform (FFT) algorithm.
} 


\section{Diffusion Steps: Lateral Translation and Rotations}

Monte Carlo simulations do not include realistic time scales, but allow one to describe average thermodynamic properties. For this study this means that one needs to define carefully the underlying diffusion steps. Diffusion steps have already been introduced in section 3.1.4. These are possible rotations and translational diffusion of two lipids (see fig. 3.2). If one is just interested in thermodynamical averages it does not matter how one gets to an equilibrium state. Non-physical diffusion steps of distant lipids still allow one to predict correctly excess heat capacity profiles. In the study of diffusion processes, however, this is not appropriate. Therefore, just lipid exchange of two neighboring lipids is allowed. This does not mean that one has found a relation between Monte Carlo time and real time. This relation can only be found through comparison of experiments and numerical results.

\section{Introduction of Microviscosity in the Model}
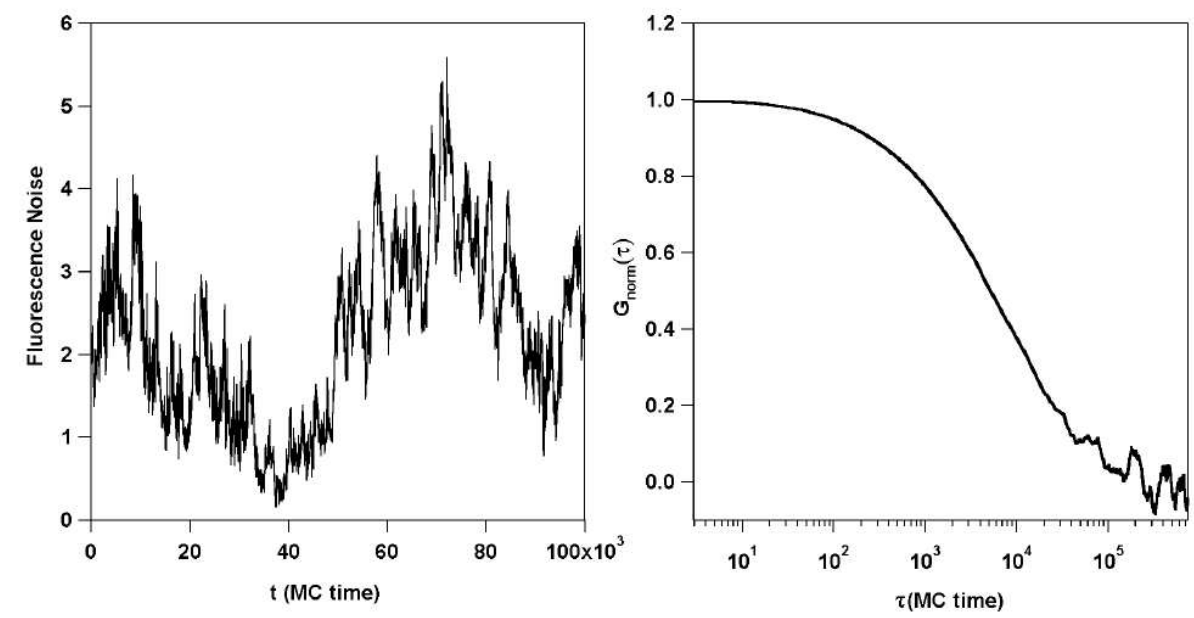

Figure 3.5: Fluorescence intensity trace and autocorrelation. Displayed are (left) a fluorescence intensity trace from a simulation of a FCS experiment and (right) the corresponding autocorrelation profile.

In the Monte Carlo simulation one has to implement the fact that diffusion in a pure ordered lipid environment is about a factor of 80 slower than in a membrane with only disordered lipids. To achieve this a microviscosity is defined in the model, which relates the frequency of acceptance of a diffusion step in a pure disordered lipid environment with the one in a pure ordered lipid environment:

$$
r\left(f_{c, o}\right)=r_{0} \cdot e^{-f_{c, o} \frac{\Delta E}{k_{B} T}},
$$

with $r\left(f_{c, o}\right)$ being the rate function describing the probability of the acceptance of entering the diffusion step routine ${ }^{7}, r_{0}$ the probability of entering the diffusion routine of the MC simulation in a pure disordered lipid membrane, $f_{c, o}$ the fraction of ordered lipid chains, $k_{B}$ the Boltzmann constant, $T$ the temperature and $\Delta E$ the activation barrier of diffusion in an all

\footnotetext{
$\overline{7}$ The diffusion step routine is where one calculates the free energy difference of the old and new configuration after a rotation or lateral movement. In addition, one decides whether to accept the new configuration. This routine is entered with a probability corresponding to $r\left(f_{c, o}\right)$.
} 
ordered lipid environment. For the determination of $f_{c, o}$ takes into account not only the lipid chains which are involved in a diffusion step, but also their nearest neighbors. The activation barrier needs to be calibrated by comparison of simulation results with experimental data.

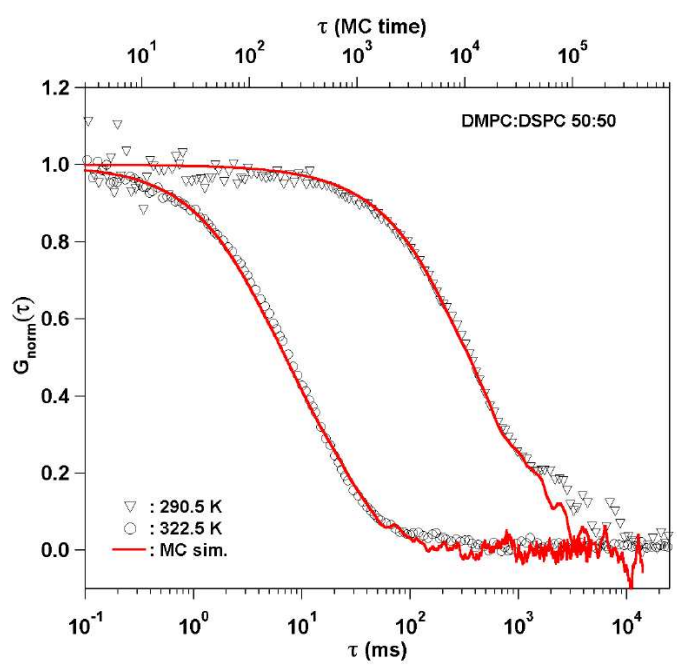

Figure 3.6: Calibration of exchange activation barrier. In eq. 3.16 a rate function is introduced which describes the activation difference of diffusion between an all ordered and an all disordered environment. The value of $\frac{\Delta E}{k_{B} T}$ is determined to be $\frac{\Delta E}{k_{B} T} \approx 4.25$. This is done by fitting experimental autocorrelation profiles of solid ordered and liquid disordered membranes using results from simulations of FCS experiments at the given temperatures. Here, experimental results from measurements on DMPC:DSPC 50:50 at $290.5 \mathrm{~K}$ ("so") and $322.5 K(" l d ")$ and the corresponding simulation results with $\frac{\Delta E}{k_{B} T}=4.25$ are shown.

As explained below, fluorescence intensity traces (see sec. 2.3) can be calculated from the Monte Carlo simulations and further analyzed by an autocorrelation (see fig. 3.5). The autocorrelation curve gives information about the diffusion processes (see sec. 2.3). Performing FCS experiments on lipid membranes either in the "ld" or "so" state results in two experimental curves with different diffusion times (see fig. 3.6). MC simulations at the chosen temperatures again allow the calculation of two autocorrelation curves. The parameter $\frac{\Delta E}{k_{B} T}$ needs to be adjusted so that the two autocorrelation profiles fit the respective experimental ones. This is the case if $\frac{\Delta E}{k_{B} T}$ equals approximately 4.25 .

\section{Laser Focus and Fluorescence Intensity}

In the FCS experiment fluorescence labels diffuse through a confocal volume, which is assumed to have a Gaussian cross section (see section 2.3). This needs to be mimicked by the MC simulation.

A given number of lipid chains are randomly labeled as being a fluorescent marker (see fig. 3.7). It is excluded that one lipid has two marked chains. In an experimental realization fluorescence dyes have different physical properties than the lipids and their chains. This, however, is not considered in this model. In FCS experiments the concentration of fluorescence 
markers is low ${ }^{8}$. Hence, the perturbation of the lipid membrane because of the labels is small.

Further, it is assumed that the fluorescence life time is shorter than a typical diffusion step. A typical fluorescence life time is around $4 \mathrm{~ns}$, whereas the mean time to move in an "ld" environment a distance of one lipid diameter is about 30 ns (Böckmann et al., 2003). In the simulation this means that a fluorescence marker emits immediately after excitation.

As has been mentioned before a Gaussian cross section of the confocal volume is assumed (see also fig. 3.7). Throughout a simulation fluorescence markers diffuse in the simulation matrix. The fluorescence signal which is emitted by a single lipid marker is calculated by:

$$
I(\vec{r})=I_{0} \cdot e^{-\frac{\left(\vec{r}-\vec{r}_{0}\right)^{2}}{2 \sigma^{2}}},
$$

where $I(\vec{r})$ is the fluorescence intensity of a single marker at physical position $\vec{r}, I_{0}$ the intensity of a marker in the laser focus origin, $\vec{r}_{0}$ is the focus origin and $\sigma$ determines the size of the laser focus. The total signal is the sum of the intensities of the individual markers. The fluctuations in fluorescence intensity are evaluated by means of an autocorrelation in which the exact intensity does not play a role. Therefore, $I_{0}$ can arbitrarily be chosen to be any number bigger than 0 . Here, $I_{0}$ is set to one. A typical fluorescence trace and its autocorrelation curve are shown in fig. 3.5.

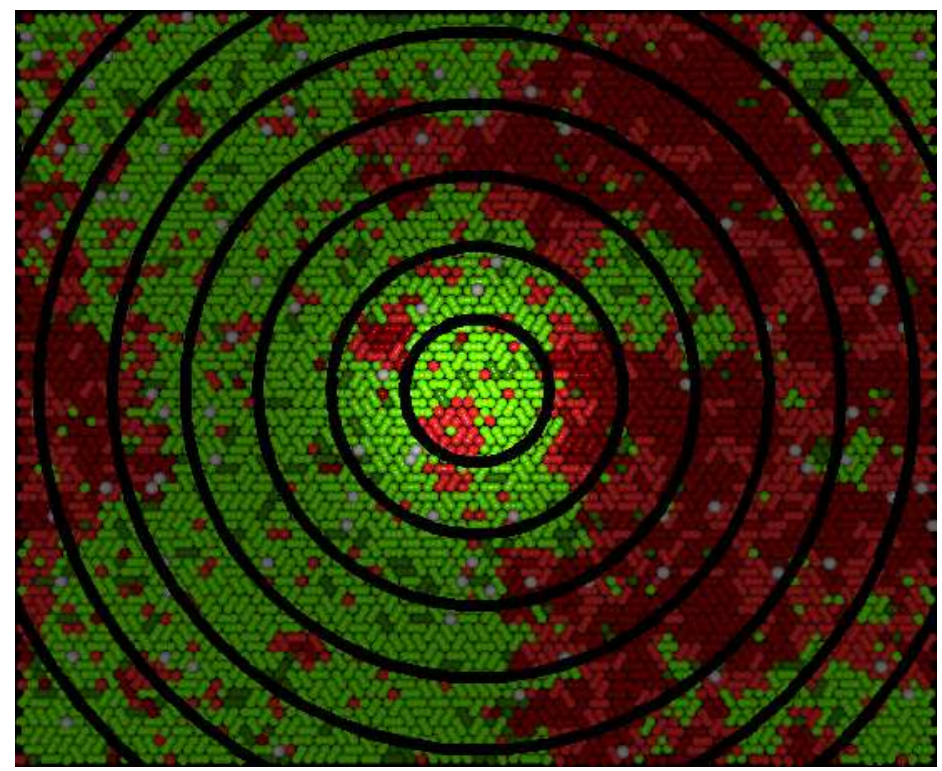

Figure 3.7: Laser Focus in the FCS Simulation. Depending on temperature and DMPC:DSPC ratio a certain domain structure can be found (red $\widehat{=}$ ordered, green $\widehat{=}$ disordered, light colors belong to DMPC and dark to $D S P C$ chains). A focus with Gaussian cross section (concentric circles) is introduced and a defined number of lipid chains are labeled (white dots). Conducting FCS simulations one can monitor fluorescence intensity traces. The dependence of diffusion processes on temperature and composition can be studied.

\footnotetext{
$\overline{{ }^{8} \text { Hac (2004) used a lipid to label ratio of } 40} \cdot 10^{6}: 1$.
} 


\section{Virtual Extension of the Diffusion Matrix}

Eq. 3.17 shows that one needs to know the physical position of a label on the matrix. Periodic boundary conditions are introduced (see sec. 3.1.2). For a diffusing particle this means that e.g. diffusing out of the matrix at the right side, it enters again on the left side. Because of this the diffusion is not reflected correctly leading to a bias in the autocorrelation profile (data not shown). This effect is negligible if the focus size is small in comparison to the matrix size. Since domain sizes might be of the order of the matrix size, underestimating one or another domain ( "ld" or "so") is possible if the focus size is too small. Therefore, the focus size needs to be of the size of the matrix. The problem with diffusion over boundaries, however, can be solved through introducing "virtual matrices". For diffusion processes the simulation matrix is extended through itself in all directions. This means that a label diffusing out of the right side of the matrix still enters it on the left side again. This allows to calculate the free energy change of a diffusion step. However, for describing diffusion the direction of the diffusion step is followed. The physical position of the marker is changed with respect to the one it has in the original simulation box. The physical position is the one it has in the original simulation matrix plus an extension. The extension depends on the diffusion direction and is related to the size of the simulation matrix. The index number of the label on the original matrix is $(N, M)$, where $N$ and $M$ are integers between one and the matrix size. For calculating the physical position one needs to use the index $(N+n \cdot M W, M+m \cdot M W)$, with $M W$ being the matrix size and $n, m$ integers between $-A \max$ and Amax. Amax is a chosen integer which defines the number of "virtual matrices". If Amax equals 2 it means that on each side of the simulation box two more matrices are attached. When diffusing over boundaries, $n$ and $m$ can only change by \pm 1 . The sign depends on the direction of diffusion. In principal a label can diffuse back into the focus by repeatedly leaving the matrix in one direction. This is because a label which diffuses from a virtual matrix with $n=A \max$ out of the box on the right side would enter it again on the left side with $n=-A \max$.

Using this method one can use a laser focus that covers the whole matrix and the contribution of the diffusion in domains of a certain physical state is not underestimated in comparison to domains of the other physical state. In addition the focus size remains small in comparison to the whole extended simulation matrix. Concerning fig. 3.7 this means that the white spheres which mark a labeled lipid chain are considered to be in the same matrix in relation to the calculation of the free energy of the system, but they might be in different virtual matrices or the original simulation matrix concerning the diffusion processes. A marker which stays in one of the virtual matrices is more distant from the laser focus, than one which resides in the actual simulation box. The intensity contribution to the whole fluorescence signal is also smaller.

After the equilibration process, a defined number of lipid chains are randomly assigned to be fluorescence markers. Integers between -Amax and Amax are randomly appointed to $n$ and $m$. The physical position of the label is in either the original simulation matrix or in one of the virtual matrices.

\subsubsection{Evaluation of Local Fluctuations}

One advantage of Monte Carlo simulations of lipid membrane systems in comparison with analytical mean field models is that they yield information about the structural properties of the lipid membrane. These can be indirectly studied by analyzing the diffusion processes 
in an equilibrated system, but also a direct visualization of the lipid chain state distribution on the matrix is possible. After a Monte Carlo cycle the distribution of lipid chains can be saved in a file. Later, this information can be visualized using ray tracing or other graphical programs (see the left picture of fig. 3.8).
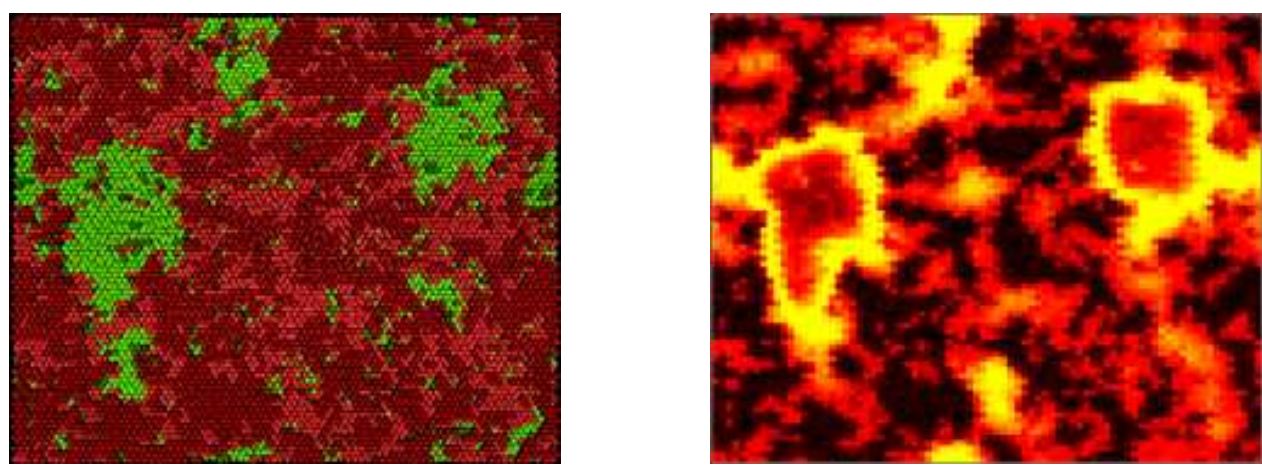

Figure 3.8: Lipid chain state distribution and local fluctuations. (left) A snapshot of the lipid chain state distribution during a MC simulation is drawn. Dark red or green colors stand for a chain of a DSPC lipid and light colors for DMPC lipid chains. Red indicates that the chain is in an ordered configuration, whereas green reflects a disordered chain. (right) Local fluctuations in chain state were calculated for the matrix configuration as seen in the left panel. Large fluctuations are represented through a bright yellow, while small fluctuations are black.

Not only can the static distribution of the lipid chain state be studied, but also local fluctuations in chain state, by turning off the diffusion steps during a simulation. For a defined number of MC cycles the states of a chain are monitored, a state parameter $S(i, j, t)$ is assigned and the local fluctuation of a chain $f(i, j)$ is defined as:

$$
f(i, j)=\left\langle S(i, j, t)^{2}\right\rangle-\langle S(i, j, t)\rangle^{2} .
$$

Assigning values of $S(i, j, t)=1$ in the case of a disordered and $S(i, j, t)=0$ for an ordered chain, fluctuations can be calculated, which lie between 0 and 0.25 . The higher $f(i, j, t)$ is the higher the fluctuation. Introducing a color code (black=low, red=intermediate and yellow $=$ high fluctuations) one gets pictures like the one in the right panel of fig. 3.8.

\subsubsection{Simulation of Temperature Jump Experiments}

In section 2.2 pressure perturbation calorimetry was introduced. Changing the pressure on a lipid suspension one can jump from a pure membrane phase of the lipid membrane ( $s o$ " or "ld") into a domain coexistence region. A change in pressure is equivalent to a change in temperature. A temperature jump can easily be simulated in a Monte Carlo simulation. One starts from an equilibrated system and at a given Monte Carlo cycle one changes the temperature of the system. This results in a change of the matrix distribution and can be quantitatively studied by analyzing the time course of the number of fluid chains or the enthalpy (see fig. 3.9). This time course depends on the relaxation process. These jumps show a lack of statistical accuracy in comparison to the autocorrelation of enthalpy fluctuations. Many temperature jump simulations have to be performed so that one gets a statistically relevant result. 


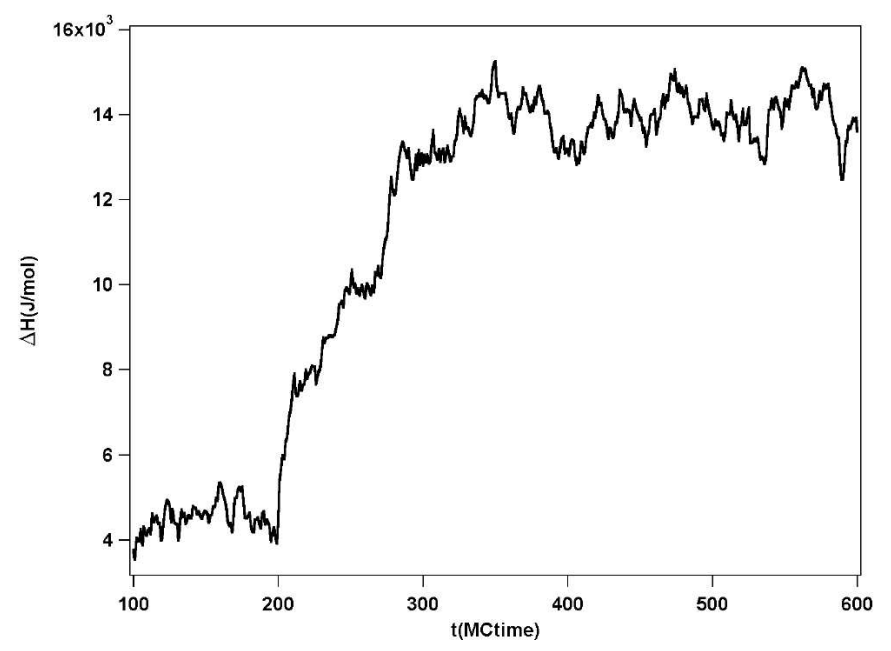

Figure 3.9: Enthalpy course after a temperature jump. The time-dependent development of the enthalpy of a lipid membrane after a sudden change in temperature in a simulation is shown.

\subsection{Relaxation Times in Membranes: An Analytical and Nu- merical Approach}

As mentioned in sec. 1.4 many studies about relaxation processes in artificial membranes are based on detection methods which are not founded on thermodynamical grounds. In this work the measured relaxation behavior is described by thermodynamical means. This includes measurements using pressure jump calorimetry (see sec. 3.9), as well as theoretical explanations based on statistical thermodynamics.

In this section it is shown that the measured relaxation processes can be related to fluctuations in enthalpy. Based on non-equilibrium thermodynamics a proportionality between relaxation time and heat capacity can be derived. The presented theory has been introduced by (Grabitz et al., 2002). Monte Carlo simulations of a two state model verify the analytical results (Ivanova, 2000; Grabitz et al., 2002).

\section{An Analytical Approach}

The distribution of enthalpy states $P(H-\langle H\rangle)$ is related to the Gibbs free energy of a state $(G(H-\langle H\rangle))$ (Lee and Kosterlitz, 1991):

$$
G(H-\langle H\rangle)=-R T \ln P(H-\langle H\rangle)+\text { const. }
$$

where $\langle H\rangle$ is the mean enthalpy, $R$ the gas constant and $T$ the temperature.

Assuming that the distribution of enthalpy states is Gaussian ${ }^{9}$ :

$$
P(H-\langle H\rangle)=\frac{1}{\sigma \sqrt{2 \pi}} e^{-\frac{(H-\langle H\rangle)^{2}}{2 \sigma^{2}}},
$$

\footnotetext{
${ }^{9}$ This assumption is always true for systems which undergo continuous phase transitions. It loses, however, its validity for first order transitions at their transition midpoint, but might be still true away from the transition midpoint.
} 
where $\sigma^{2}=\left\langle H^{2}\right\rangle-\langle H\rangle^{2}$. Further evaluation of eq. 3.19 leads to:

$$
G(H-\langle H\rangle)=R T \frac{(H-\langle H\rangle)^{2}}{2 \sigma^{2}}+\text { const. }
$$

Using expression 3.21 and $S(H)=\frac{H-G}{T}$ one finds:

$$
S(H-\langle H\rangle)=\frac{H-\langle H\rangle}{T}-\frac{R(H-\langle H\rangle)^{2}}{2 \sigma^{2}}-\frac{\text { const. }}{T} .
$$

Assuming that the quadratic term is the leading one, the linear and constant term can be neglected. The entropy can be seen as a harmonic potential:

$$
S(H-\langle H\rangle)=-\frac{R(H-\langle H\rangle)^{2}}{2 \sigma^{2}} .
$$

In non-equilibrium thermodynamics ${ }^{10}$ one introduces thermodynamical forces $X_{i}$ which drive a system back to its equilibrium. It can be shown that these thermodynamical forces are the second derivative of the entropy with respect to fluctuations $\alpha_{i}$ of the system:

$$
X_{i}=\sum_{j}\left(\frac{\partial^{2} S}{\partial \alpha_{i} \partial \alpha_{j}} \alpha_{j}\right) .
$$

Further, in non-equilibrium thermodynamics one considers time dependent changes of the fluctuations, the fluxes:

$$
J_{i}=\frac{\partial \alpha_{i}}{\partial t}=\sum_{j} L_{i j} X_{j}
$$

with $L_{i j}$ being phenomenological coefficients. Thermodynamical fluxes are, therefore, related to the thermodynamical forces.

For artificial membrane systems it can be shown that in the melting transition changes in volume, enthalpy and area are proportional to each other (Heimburg, 1998; Ebel et al., 2001). Hence, it is sufficient to consider one independent fluctuation $\alpha=H-\langle H\rangle$. Using eqs. 3.23 and 3.24 one finds that the related thermodynamical force is given by:

$$
\begin{aligned}
X(H-\langle H\rangle) & =\left(\frac{\partial^{2} S(H-\langle H\rangle)}{\partial(H-\langle H\rangle)^{2}}\right)_{0}(H-\langle H\rangle) \\
& =-\frac{R(H-\langle H\rangle)}{\sigma^{2}} .
\end{aligned}
$$

Using the flux of enthalpy $J$ back to equilibrium one obtains:

$$
J(H-\langle H\rangle)=\frac{\partial(H-\langle H\rangle)}{\partial t}=L X(H-\langle H\rangle)=-L \frac{R(H-\langle H\rangle)}{\sigma^{2}} .
$$

The differential equation 3.27 can be easily solved and one finds that the time dependent fluctuations in enthalpy can be described by a single exponential function:

$$
(H-\langle H\rangle)(t)=(H-\langle H\rangle)(0) e^{-\frac{R L}{\sigma^{2}} t} .
$$

\footnotetext{
${ }^{10}$ For an introduction see for example the excellent textbook by Kondepudi and Prigogine (1998).
} 
The factor $\frac{\sigma^{2}}{R L}$ is in units of time, which allows one to define the relaxation time $\tau$ :

$$
\tau=\frac{\sigma^{2}}{R L}
$$

The fluctuation dissipation theorem (Hill, 1962) relates the fluctuations in enthalpy to the heat capacity of the system:

$$
c_{p}=\frac{\left\langle H^{2}\right\rangle-\langle H\rangle^{2}}{R T^{2}}=\frac{\sigma^{2}}{R T^{2}} .
$$

Using eq. 3.29 and 3.30 one finds a linear relation between heat capacity and relaxation time:

$$
\tau=\frac{T^{2}}{L} c_{p}
$$

The proportionality factor between the relaxation time and the heat capacity is $\xi_{p} \equiv \frac{T^{2}}{L}$. The phenomenological coefficient $L$ has to be obtained from experiments. No theoretical justification can state that the phenomenological coefficient is the same for all membrane systems. Grabitz et al. (2002), however, have found that in their experiments on MLVs, it seemed to equal independent of the lipid species used.

In the same work, and also in Ivanova (2000), results from Monte Carlo simulations were presented to show the validity of the proportionality between heat capacity and the relaxation times. The model they used differs slightly in comparison to ours. Ivanova (2000) arranges lipids on a triangular lattice and not the lipid chains. The whole lipid can be either in an ordered or disordered state. Again just nearest neighbor are considered. Relaxation times were obtained from single exponential fits of autocorrelated enthalpy traces. In the following, our model is used to verify these findings and the linear relation between heat capacity and relaxation times.

\section{Monte Carlo Simulation Approach}

In chapter 3.1.2 a two state model is introduced to describe the phase behavior of a two component lipid system. This model also allows one to characterize the melting profile of a one component lipid system. The number of parameters needed in the simulations reduces from ten to three. One needs to define the enthalpy, $\Delta H$, and entropy, $\Delta S$, change between the ordered and disordered system and the interaction parameter between ordered and disordered lipid chains $\omega$.

The following results were obtained from simulations with the parameters $\Delta H=$ $13168 \mathrm{~J} /($ mol chain $)$ and $\Delta S=44.31 \mathrm{~J} /($ mol chain $K)$. These are the ones obtained from DSC measurements on multilamellar DMPC vesicles (see sec. 3.1.3). The ordered-disordered interaction parameter was set to $\omega=1311.79 \mathrm{~J} /($ mol chain $)$. Three enthalpy traces at different temperatures are shown in fig. 3.10. One temperature equals, another one is above and a third one is below the transition midpoint temperature.

Already in these traces one can see differences in the time scale of the fluctuation depending on where one is in the melting transition. The trace which is a result of a simulation at the transition midpoint shows the slowest fluctuations compared to the traces above and below the transition midpoint. An autocorrelation of the enthalpy fluctuations allows a further analysis of the data (see eq. 3.15). 


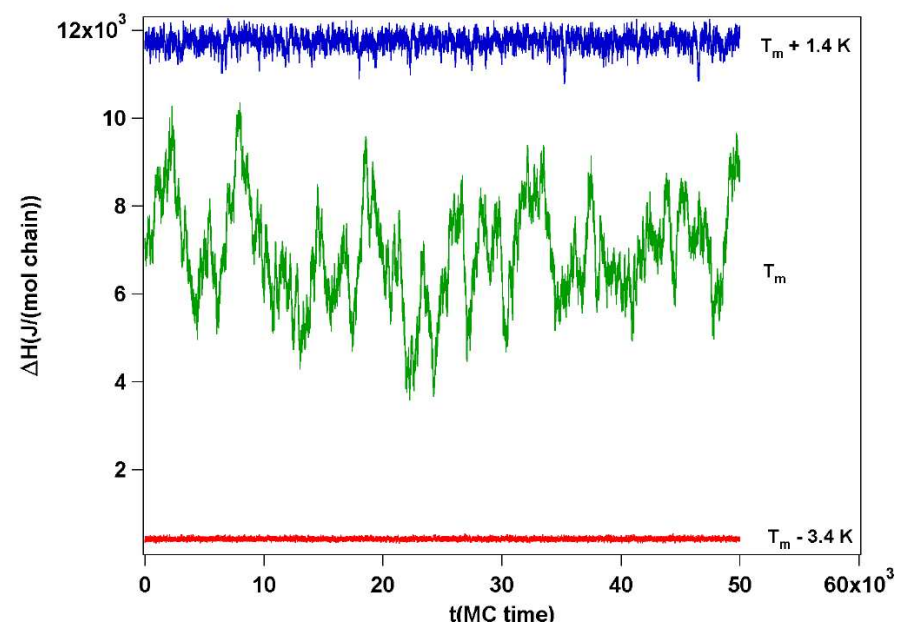

Figure 3.10: Enthalpy fluctuations. Simulations of a one component system with $\Delta H=13168 \mathrm{~J} /(\mathrm{mol}$ chain $)$, $\Delta S=44.31 \mathrm{~J} /($ mol chain $\mathrm{K})$ and $\omega=1311.79 \mathrm{~J} /($ mol chain $)$ at different temperatures were performed. The fluctuations in enthalpy at different temperatures differ in strength and in the time scale of the fluctuations.

In fig. 3.11 the results of the autocorrelations of the three enthalpy traces from fig. 3.10 are shown. The autocorrelation profile of the trace at the transition midpoint decays at longer times than the profiles below or above the transition midpoint. The relaxation process is slower than in the other two cases. The autocorrelation profiles can be fitted to a single exponential decay $\left(e^{-\frac{t}{\tau}} ;\right.$ dashed black lines $)$.

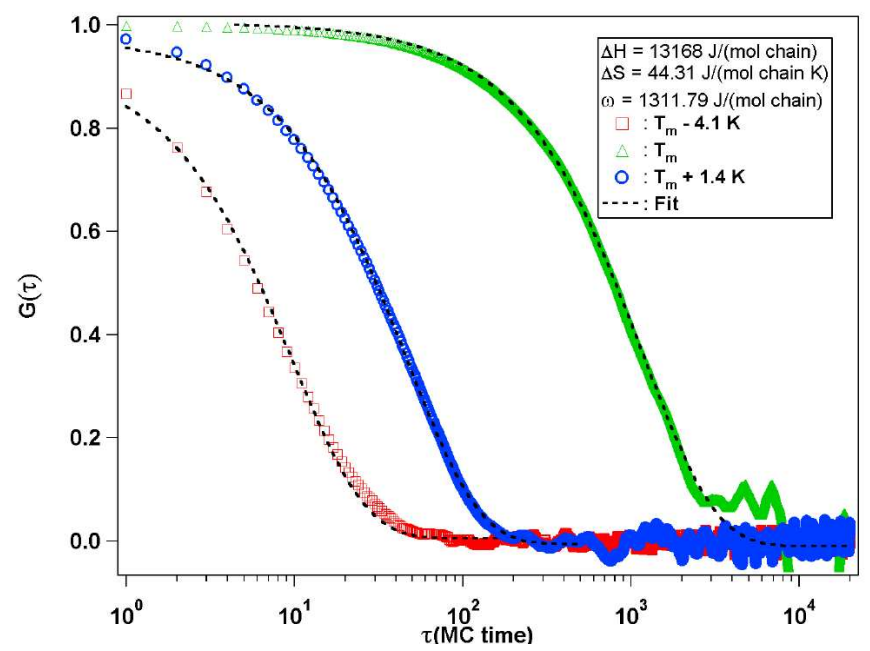

Figure 3.11: Autocorrelation profiles of the enthalpy traces as shown in fig. 3.10. The decay of the autocorrelation curves happens on different time scales. This means that the relaxation times differ depending on temperature. Dashed black lines are respective single exponential fits.

The Monte Carlo simulation allows one to perform simulations with various ordereddisordered interaction parameters. From these simulations one can record enthalpy traces at 


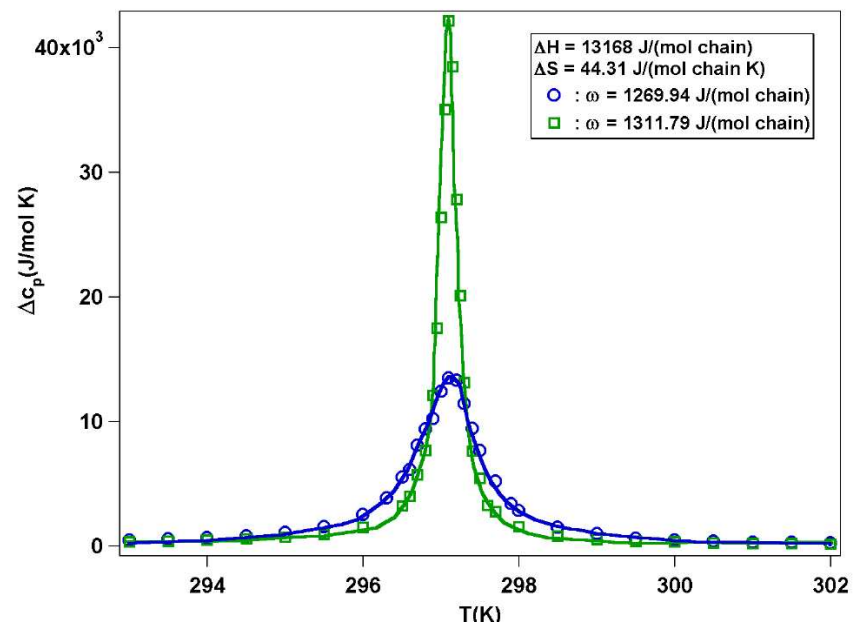

Figure 3.12: Simulated heat capacity profiles for a one component lipid system with $\Delta H=$ $13168 \mathrm{~J} /($ mol chain $), \Delta S=44.31 \mathrm{~J} /($ mol chain $K)$. The interaction parameter between ordered and disordered chains differs. The two values chosen were $\omega=1269.94 \mathrm{~J} /($ mol chain $)$ and $\omega=1311.79 \mathrm{~J} /($ mol chain $)$.

various temperatures. Since heat capacities are related to the strength of enthalpy fluctuations (fluctuation-dissipation theorem; see eq. 3.13) one obtains $\Delta c_{p}$-profiles. In fig. 3.12 two profiles simulated with two different interaction parameters are shown $(\omega=1269.94 \mathrm{~J} /($ mol chain $)$ and $\omega=1311.79 \mathrm{~J} /(\mathrm{mol}$ chain $))$. The entropy and enthalpy changes were again $\Delta H=$ $13168 \mathrm{~J} /($ mol chain $)$ and $\Delta S=44.31 \mathrm{~J} /($ mol chain $K)$. A reduction in interaction parameter results in a broadening of the heat capacity curve. In addition, the heat capacity maximum decreases. The parameter $\omega$ describes the cooperativity of the melting transition. The higher the parameter the more cooperative the transition and the smaller the half-width of the profile. The area below both curves is the same. It gives the transition enthalpy $\Delta H$.
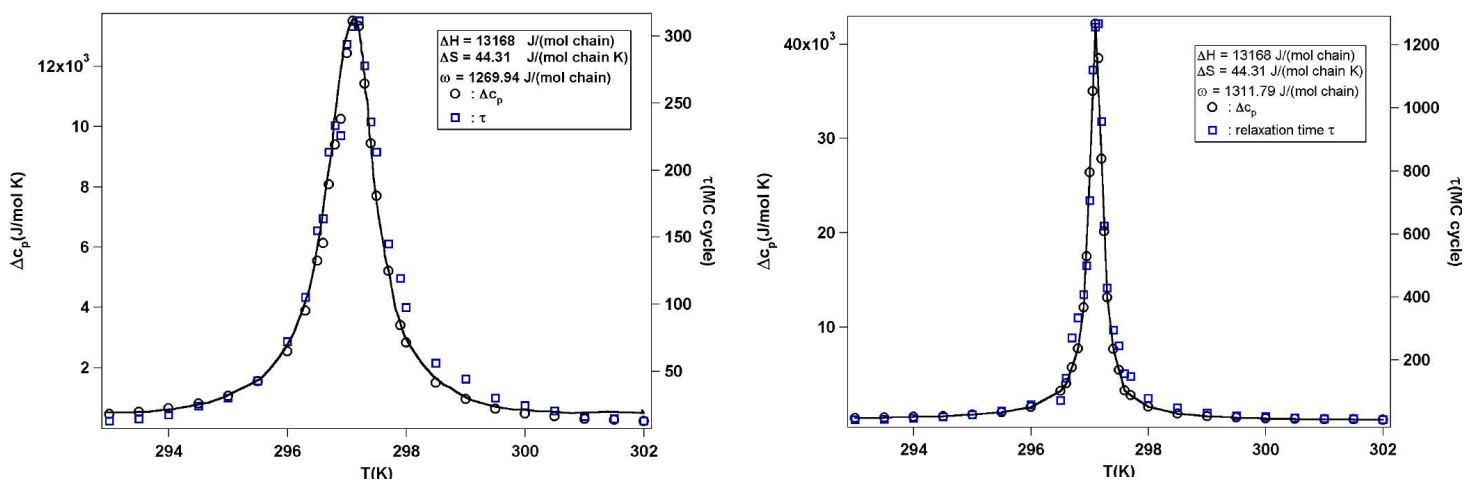

Figure 3.13: Comparison between heat capacity profiles shown in fig. 3.12 and the calculated relaxation times. One finds that the excess heat capacity and the relaxation times are proportional to each other. The scaling of the heat capacity and relaxation time axes differ in the two graphs. This is in accordance with the finding of the analytical non-equilibrium thermodynamics approach.

Evaluating the enthalpy traces through an autocorrelation and fitting it with the assumption of an exponential decay one obtains relaxation times for the different simulations at 
all temperatures. In the two simulated cases the relaxation times are proportional to heat capacity profiles (fig. 3.13).

This is an important finding since the shapes of the curves differ. Also the maximal relaxation times differ from each other. The more pronounced the transition profile the slower the relaxation process.

These numerical results are in accordance with the analytical finding that relaxation times and heat capacity are proportional to each other (see eq. 3.31; (Grabitz et al., 2002)).

Grabitz et al. (2002) were the first ones to present a thermodynamical theory which explains the experimentally found proportionality between relaxation time and heat capacity. In order to prove their finding they performed pressure jump calorimetry on various lipid membrane systems. They concluded that the proportionality is true for all one component lipid systems. This was also valid for a multilamellar DMPC/cholesterol system. They also claimed that the phenomenological constant $L$ stays the same for all systems.

It is non-trivial to claim that more complex systems still behave in the same way. For this reason measurements on lipid systems with different concentrations of peptides, anesthetics and neurotransmitters were performed. The results of these are presented in chapter 4 . The study of relaxation phenomena is extended to binary systems using the numerical approach. 


\section{Chapter 4}

\section{Results}

In this chapter experimental and simulation results are presented. First the validity of the introduced numerical model for the description of the phase behavior of DMPC:DSPC lipid membranes is shown. After that the model is successfully used for the description of diffusion experiments with fluorescence correlation spectroscopy (FCS). In the following section experimental heat capacity profiles of different artificial membrane systems are presented. In most of the cases relaxation measurements were performed on these systems, too. Monte Carlo (MC) simulations help to deepen the understanding of the measured relaxation behaviors. They also allow one to extend the studies to lipid mixtures. So far pressure perturbation experiments have not been successful on binary lipid mixtures. The final section is a pure numerical analysis of fluctuations and domain sizes of the DMPC:DSPC lipid system.

\subsection{Experimental and Simulated Phase Diagrams of DMPC:DSPC}

A two state model of lipid chain states with nearest neighbor interactions is analyzed by means of MC simulations. Sugar et al. (1999) used this model for the description of the phase behavior of a two component lipid system, in this case DMPC:DSPC. As has been pointed out earlier in the text fluctuations in enthalpy are related to heat capacity. Therefore, monitoring enthalpy with time at different temperatures allows one to calculate the heat capacity at various temperatures. With the right set of nearest neighbor interaction parameters one is able to fit measured heat capacity profiles ${ }^{1}$.

Calorimetric measurements were done by T. Schlötzer on suspensions of multilamellar vesicles (MLVs) with the Microcal MCS as described in Schlötzer (2002) at a scan rate of $5 \frac{K}{h}$. Simulations were performed on normal PC workstations with Pentium III processors.

Because of finite size effects the size of the simulation matrix has to be varied depending on the simulated mixture. An increase of the matrix size beyond a certain threshold size does not change the calculated heat capacity profile. At sizes smaller than the critical size one clearly sees a dependence on matrix size. Minimum matrix sizes for the different DMPC:DSPC mixtures are given by Sugar et al. (1999). These minimum sizes depend on the molar ratios of the two lipid species. We chose sizes bigger than the minimum ones. In the case of the mixtures $70: 30,60: 40,50: 50,40: 60$ and $30: 70$ the matrix size was set to 60 chains $\times 60$ chains. It

\footnotetext{
${ }^{1}$ These are results which were already published in my master's thesis. For reasons of completeness and because of the importance of the model it is given again.
} 

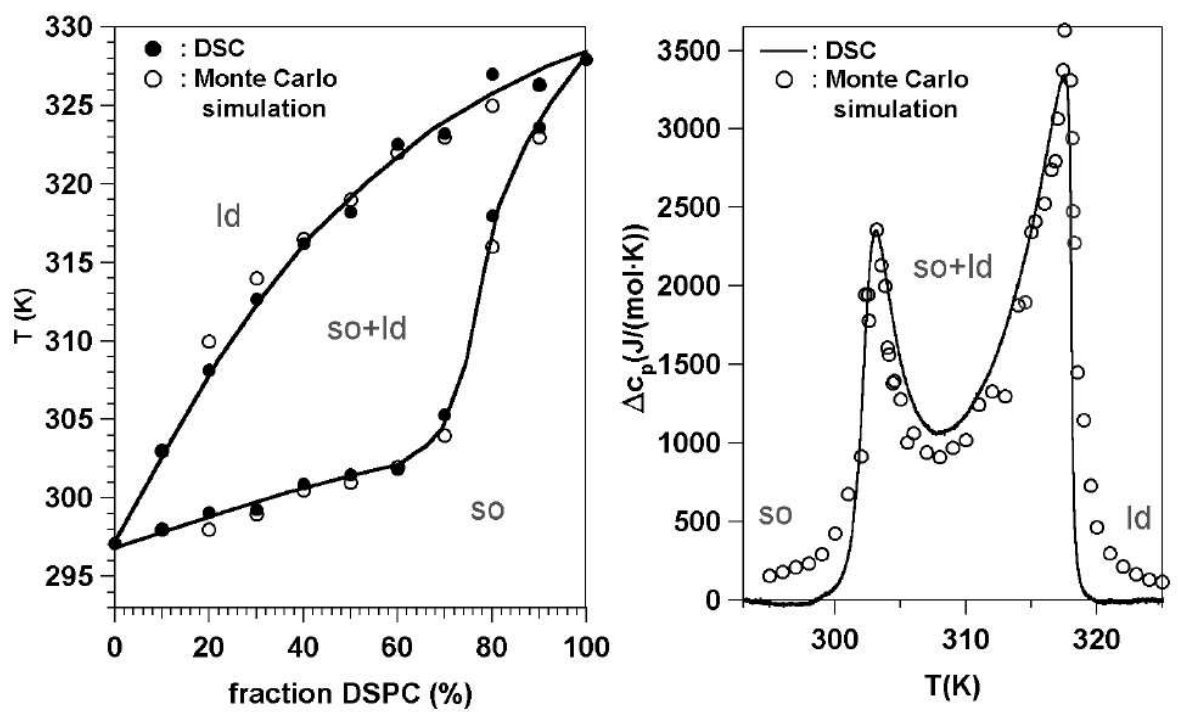

Figure 4.1: Experimental and theoretical phase diagrams and heat capacity profiles. (left) The phase diagrams of the DMPC:DSPC lipid system were obtained by analyzing excess heat capacity profiles from DSC experiments (filled circles) and MC simulations (open circles). (right) The calculated heat capacity values (open circles) describe the measured experimental profile (solid curve) of an equimolar DMPC:DSPC mixture. This is also true for other DMPC:DSPC mixtures (data not shown; (Hac et al., 2005; Seeger et al., 2005)). The experiments were performed by T. Schlötzer (Schlötzer, 2002).

was increased to 100 chains $\times 100$ chains for the mixtures $90: 10,80: 20,20: 80$ and $10: 90$. For the one component systems they were chosen to be 350 chains $\times 350$ chains (DMPC) and 300 chains $\times 300$ chains (DSPC).

Since high values in heat capacity are related to strong fluctuations in enthalpy (fluctuationdissipation theorem; see eq. 3.13) one needs to collect long traces of enthalpy fluctuations in these cases. Therefore, the number of MC cycles was varied for different temperatures. In a temperature region of $\pm 4 K$ around the maxima of the heat capacity profile $10000 \mathrm{MC}$ cycles were performed to find the equilibrium position, followed by $100000 \mathrm{MC}$ cycles in the equilibrium. At all other temperatures cycle numbers were chosen to be $7500 \mathrm{MC}$ cycles and 75000 MC cycles, respectively.

An example of a calculated excess heat capacity profile is shown in the right panel of fig. 4.1. The MC simulation fits the measured profile with reasonable accuracy. Deviations from the two curves can be noticed especially at the two flanks and at temperatures around the local $\Delta c_{p}$ minimum. The latter is more pronounced in simulations of other mixtures (data not shown). Still, the simulated profiles fit the measured curves well.

A MC simulation needs the definition of MC steps and cycles. MC steps are the elementary steps of the simulation. Here, these are changes in lipid chain state and diffusion. During a $\mathrm{MC}$ cycle the MC steps are performed in a given manner. Different definitions of MC cycles should not influence the simulation result. This requirement is known as detailed balance. We tested this using different numbers of diffusion steps in the definition of a MC cycle. No deviations were found (data not shown).

The experimental and theoretical phase diagrams were constructed from a tangent construc- 
tion at the upper and lower temperature limit of the heat heat capacity profiles. The points of intersection with the temperature axis are interpreted as marking the phase boundaries. Fig. 4.1 (left panel) shows that the experimental and the calculated phase diagrams are in agreement.

Therefore, it can be concluded that a simple two state model is sufficient to describe the phase behavior of the two component DMPC:DSPC lipid system. Since the parameters were chosen to characterize excess heat capacity profiles of MLVs the model is applied to the description of experimental diffusion investigations on stacks of supported bilayers. In the following sections the model is also used for the study of relaxation processes, perturbation experiments, domain formation and fluctuation properties.

\section{Summary}

The minimalistic two state model gives good fits to measured excess heat capacity profiles and correctly describes the phase diagram of the DMPC:DSPC lipid system.

\subsection{Diffusion Studies on Planar Membranes}

FCS was used for the investigation of diffusion processes. The diffusion of the fluorescence label TRITC DHPE was monitored in a stack of supported bilayers of DMPC:DSPC mixtures. Experiments were performed by A. Hac (Hac, 2004; Hac et al., 2005). TRITC DHPE partitions equally well into the two lipid membrane phases. These studies were accompanied by MC simulations ${ }^{2}$.

\section{The Simulation}

In the model, diffusion is mimicked by exchange of neighboring lipids or through rotations. Diffusion in a "so" membrane $\left(D \approx 5 \cdot 10^{-10} \frac{\mathrm{cm}^{2}}{\mathrm{~s}}\right)$ is about a factor of 80 slower than in a "ld" membrane $\left(D \approx 4 \cdot 10^{-8} \frac{\mathrm{cm}^{2}}{\mathrm{~s}}\right)$. This difference is modeled by introducing a microviscosity. A rate function is formulated (see eq. 3.16). An additional parameter $\frac{\Delta E}{k_{B} T}$ is needed, where $\Delta E$ defines the activation barrier of diffusion in an all ordered lipid environment. In order to define this parameter simulations were compared with results from measurements on a DMPC:DSPC 50:50 mixture at $T=290.5 \mathrm{~K}$ and $T=322.5 \mathrm{~K}$, where the membrane is in the "so" and "ld" phases, respectively. We fitted both curves using a parameter, $\frac{\Delta E}{k_{B} T}$, of 4.25 (see fig. 3.6).

Usually, simulations were performed with a matrix size of 60 chains $\times 60$ chains. Periodic boundary conditions are used in the simulation meaning that a lipid diffusion out of e.g. the right boundary of the simulation box enters the matrix from the left side again. This does not reflect the diffusion correctly and leads to errors. Virtual matrices are introduced to circumvent this problem and to follow the diffusion direction. For purposes of determining the nearest neighbors, a marked chain diffusing out of the original matrix is assumed to

\footnotetext{
${ }^{2}$ A first report about the possibility to simulate diffusion processes was already given in my master's thesis. The model, however, experienced some refinements such as the introduction of a microviscosity and of virtual matrices. In an earlier stage of the model the difference of diffusion in "so" and "ld" membranes was implemented as being purely based on the states of the four involved lipid chains. Because of a lack of experimental data simulations could not be compared with experiments in my master's thesis. The simulations were further extended in the past years.
} 


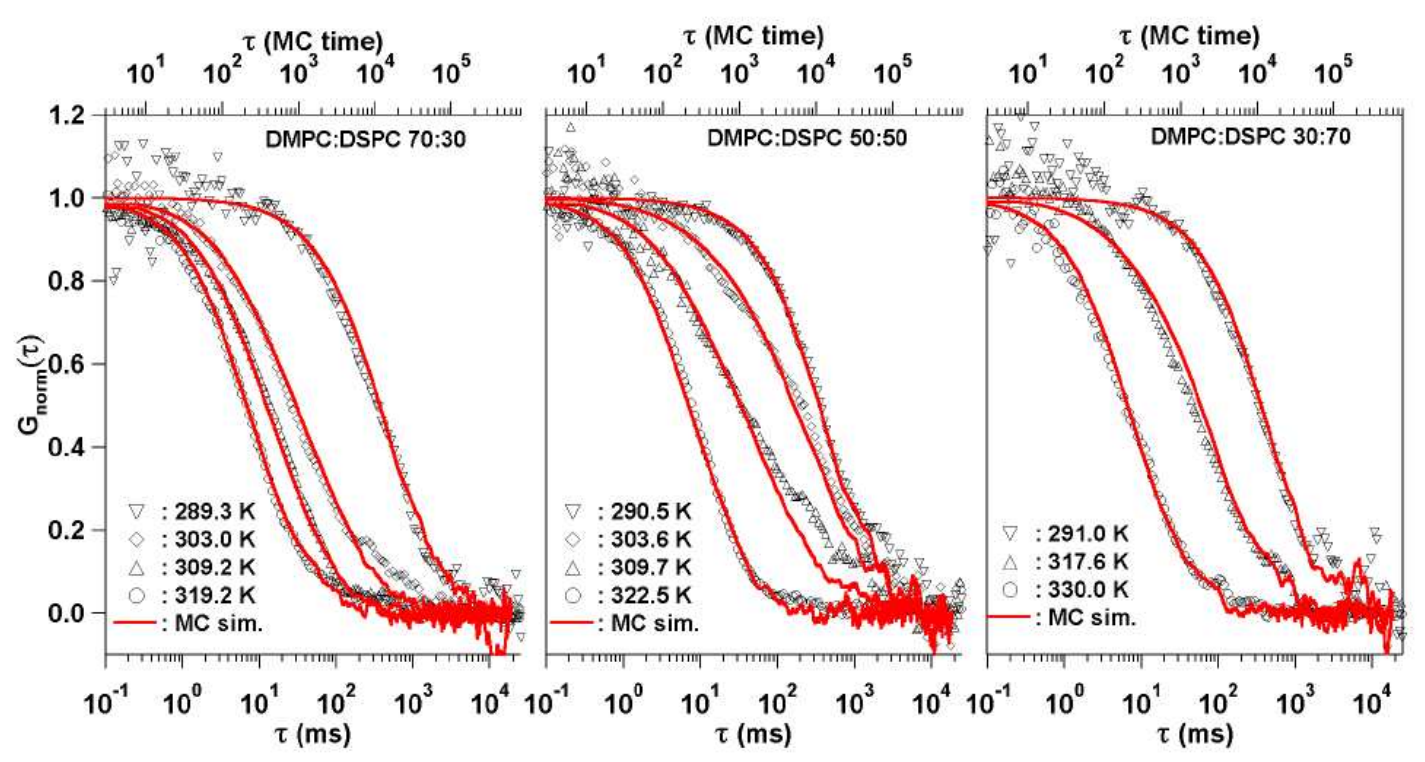

Figure 4.2: Experimental and simulated FCS autocorrelation profiles. For three DMPC:DSPC mixtures at different temperatures experimental (markers) and simulated (red, solid curves) FCS autocorrelation profiles are plotted. (left) A mixture of DMPC:DSPC 70:30 was chosen and measurements and simulations were performed at temperatures of $289.3 \mathrm{~K}, 303.0 \mathrm{~K}, 309.2 \mathrm{~K}$ and $319.2 \mathrm{~K}$. With this choice of temperatures one ensures studies of the diffusion behavior below, in and above the melting regime. (center) Measurements and simulations were also done for an equimolar DMPC:DSPC mixture. Again the temperatures were selected so that different points in the phase diagram were mapped. (right) Studies on a third system, DMPC:DSPC 30:70, were carried out as well. These were done at temperatures of $291.0 \mathrm{~K}, 317.6 \mathrm{~K}$ and $330.0 \mathrm{~K}$. The curves calculated from MC simulations describe the measured autocorrelation profiles well. It is noteworthy that they are predictions rather than fits (Hac et al., 2005).

re-enter the same matrix according to periodic boundary conditions. But for a correct treatment of the diffusion the physical position is extended by the matrix size. In the computer a marker might have the indices $(i, j)$, but it gets a physical position depending on the indices and a multiple of the matrix size. Generally an extension of $A \max =2$ was chosen, which means that two virtual matrices are extended to all sides. A label can diffuse back into the actual matrix just by diffusing in one direction. This ensures that at long times not all fluorescence markers have diffused away from the laser focus. It could be argued that boundary effects still influence the diffusion processes, but it is unlikely to be a big effect. Autocorrelation curves obtained from diffusion simulations in the pure phases could be perfectly fitted by the expected Lorentzian shape (see eq. 2.28). Simulations with different values of Amax showed deviations of the diffusion constant. Deviations were smaller than $10 \%$. Smaller box sizes might lead to an underestimation, which, however, is small. It seems that simulations using $A \max =2$ correctly mimic the diffusion processes. Focus radii equal 25 chains. Depending on temperature and therewith the diffusion rates simulations were performed over $2 \cdot 10^{6}, 6 \cdot 10^{6}$ or $8 \cdot 10^{6}$ MC cycles. $8 \cdot 10^{6}$ MC cycles were chosen in the case of slow diffusion (low temperatures). When diffusion was fast (high temperatures) fluorescence intensity traces were collected during $2 \cdot 10^{6} \mathrm{MC}$ cycles. In each case, however, 
traces of fluorescence intensity of at most $1 \cdot 10^{6}$ points were collected, meaning that the fluorescence signal was not monitored after each $M C$ cycle, but after every $2 n d, 6 t h$ or 8 th cycle.

\section{Comparison of Experiment and Simulation}

In fig. 4.2 representative simulated and measured autocorrelation profiles for three different mixtures at different temperatures are shown. Temperatures were chosen so that they correspond to different points in the phase diagram. It can be clearly seen that the calculated results describe the measured ones well. It has to be noted that these are predictions rather than fits since just the diffusion time scales in the pure "so" and "ld" membranes were used for obtaining the needed parameter $\frac{\Delta E}{k_{B} T}$ of the rate function. All other curves follow directly from the thermodynamics of the system.

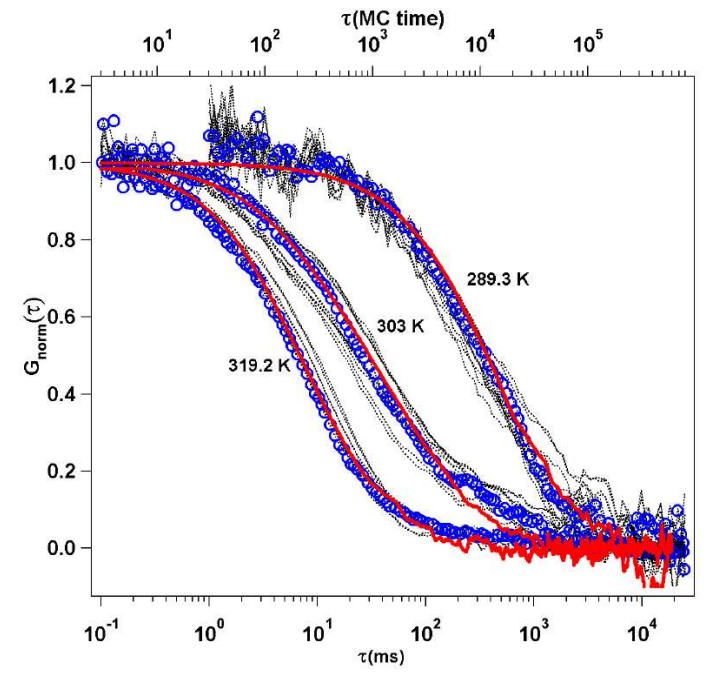

Figure 4.3: Variation of autocorrelation profiles in FCS experiments. The thin black curves and the curves shown with markers are measured autocorrelation profiles from a DMPC:DSPC 70:30 sample. The experimental profiles vary from measurement to measurement. This is possibly due to fluctuations of the sample temperature during a series of experiments. The curves indicated through the blue markers are the ones shown in fig. 4.2. The red curves display the simulated results (Hac et al., 2005).

The experimental autocorrelation profiles from different experiments vary at the same temperature (fig. 4.3) probably because of inaccuracies in the adjustment of the sample temperature. In fig. 4.3 measurement results of a DMPC:DSPC 70:30 mixture are displayed. The red curves are from MC simulations and the blue curves are the experimental results as seen in fig. 4.2. The black ones are a selection of measurements at the respective temperatures. Experimental curves in fig. 4.2 are not a biased selection. The selection is based on the received autocorrelation curves from all measurements at the same temperature. The chosen autocorrelation curves correspond to a curve with an averaged diffusion time. The simulated curves also show variations from simulations at the same mixture and same temperature. These deviations are, however, due to the statistics of the system and still result in the same diffusion time scales (data not shown). 


\section{Focus and Matrix Size}

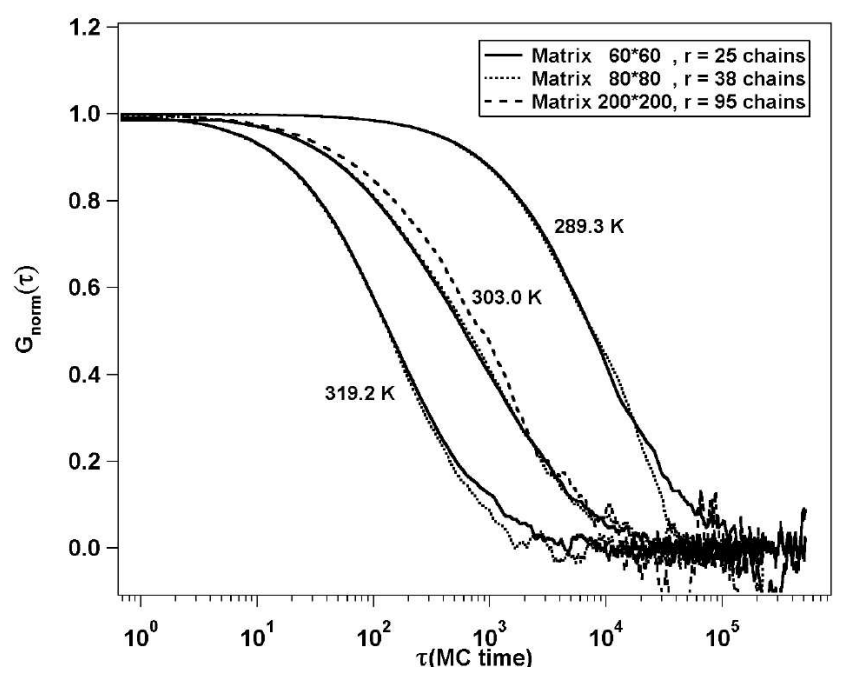

Figure 4.4: Autocorrelation profiles and dependence on focus size. Simulations with different matrix and focus sizes of DMPC:DSPC 70:30 at $T=289.3 \mathrm{~K}, T=303.0 \mathrm{~K}$ and $T=319.2 \mathrm{~K}$ were performed. The autocorrelation profiles are corrected so that each of them corresponds to a diffusion through a focus of a radius of 25 chains. Autocorrelation profiles are only slightly influenced by the system size (Hac et al., 2005).

One objection towards the simulations might be that the simulated system has a size of about $30 \mathrm{~nm} \times 26 \mathrm{~nm}$, with a focus radius of approximately $12.5 \mathrm{~nm}$. The focus radius of the experimental setup depends on the used pinhole size, but is much bigger $(r \approx 500 \mathrm{~nm})$. Therefore, simulations with different matrix and focus sizes were performed to check whether changing the matrix size influences the autocorrelation profile.

In fig. 4.4 simulated autocorrelation profiles of a DMPC:DSPC 70:30 system at three different temperatures are shown. Matrix sizes were chosen to be 60 chains $\times 60$ chains, 80 chains $\times 80$ chains and 200 chains $\times 200$ chains with the corresponding focus radii of 25 chains, 35 chains and 95 chains. The displayed autocorrelation curves are normalized to diffusion through a focus of radius 25 chains. Increasing matrix and focus size have just minor effects on the autocorrelation profile. These are due to the limited amount of fluorescence data and could probably be eliminated with longer and time consuming simulations. It can be concluded that the deviations are small even though system sizes range from $30 \mathrm{~nm} \times 26 \mathrm{~nm}$ to $100 \mathrm{~nm} \times 87 \mathrm{~nm}$. The latter case is still smaller than the experimental system, but gets into its size range. Still, size effects cannot be completely ruled out, but at least seem to be small.

\section{Timescales in the Simulation}

The good agreement between experiments and simulations allows one to use the simulations for further evaluation of additional information obtained indirectly from the experimental results. At first the following case is considered. Assuming that diffusion processes just depend on the diffusion properties of the pure lipid membrane phases one would expect that 


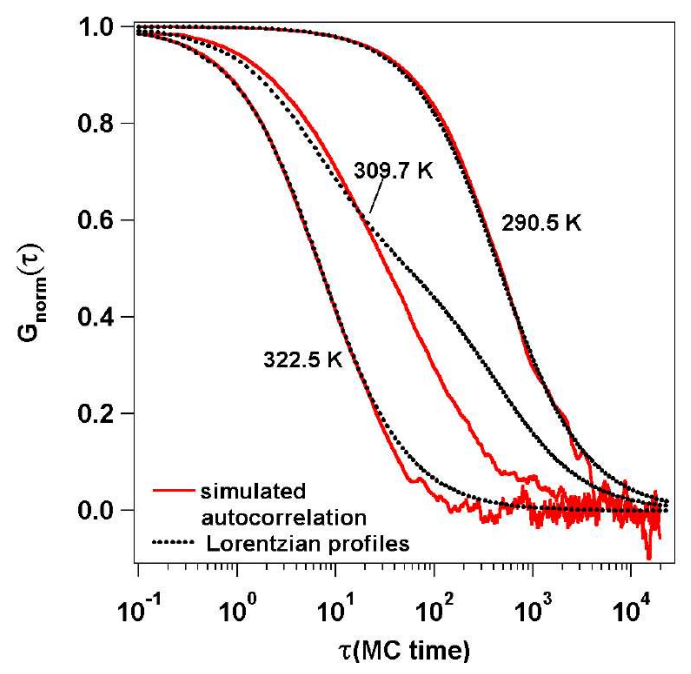

Figure 4.5: Simulated autocorrelation profiles and their comparison to a superposition of diffusion in pure "so" and pure "ld" membranes. Autocorrelation profiles as they result from MC simulations of a DMPC:DSPC 50:50 system at three different temperatures are shown (red, solid curves). The temperatures were chosen so that diffusion properties in a pure "ld", in a pure "so" membrane and in the domain coexistence regime were mapped. At temperatures of $290.5 \mathrm{~K}$ and $322.5 \mathrm{~K}$ also the respective fits (Lorentzian profiles; dashed curves) are drawn. At a temperature of $309.7 K$ the system is in a domain coexistence region. The according Lorentzian profile is a superposition of diffusion properties in the pure lipid membrane phases. The respective ratios follow from the phase diagram (Hac et al., 2005).

any autocorrelation profile in the phase coexistence regime is a superposition of the ones in the pure phases. From phase diagrams one can extract the ratio between "so" and "ld" domains. In fig. 4.5 this was done for an equimolar DMPC:DSPC mixture at $309.7 \mathrm{~K}$ (black curve). The red curves are simulated autocorrelation profiles which successfully predicted the experimental profiles. The superposition clearly deviates from this profile. For further analysis of this effect MC simulations were performed with a redefined MC cycle. The differences lie in the weighting of diffusion processes against state changes, representing differences in time scales of diffusion to fluctuations in chain state. During the time a marked lipid chain passes the laser focus it can fluctuate in chain state. Ratios between state changes and diffusion steps varied from $1: 1$ to $1: \infty$. In the last case no state changes were allowed after the equilibration. At a certain temperature the amounts of ordered and disordered lipid chains fluctuate around a mean value. In the case that there are no state changes anymore one needs to perform diffusion studies with a matrix configuration whose number of ordered and disordered chains is close to the average.

Fig. 4.6 shows results of simulations of a DMPC:DSPC 70:30 membrane with different ratios of state changes to diffusion steps. Autocorrelation profiles in the pure phases are not affected and therefore just one curve at the appropriate temperature is drawn. In the phase coexisting regime at $T=303.0 \mathrm{~K}$ autocorrelation profiles depend significantly on the ratio of state changes to diffusion steps. The more diffusion steps are performed (meaning the slower the fluctuations in chain state compared to diffusion steps in a MC cycle the later the autocorrelation curve decreases). 


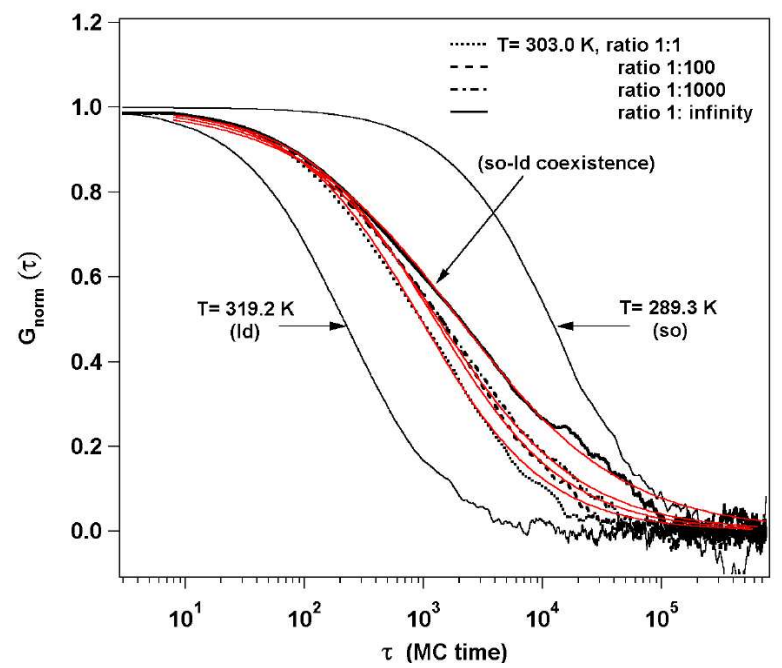

Figure 4.6: Different timescales and their influence on autocorrelation profiles. The MC simulation allows one to study the influence of the ratio between state changes to diffusion steps on the calculated autocorrelation profiles. In the extreme case no state changes were allowed. It can be seen that the less probable is a change of state during a diffusion step, the slower the decay of the autocorrelation profile. This shows that the different time scales in the simulation have an influence on the diffusion behavior. This information is also hidden in the experimental profiles (Hac et al., 2005). The red curves are the corresponding fits assuming anomalous diffusion.

Korlach et al. (1999) analyzed FCS measurements on DLPC:DPPC LUVs with fitting autocorrelation profiles using a two component fit assuming a fast and a slow diffusing component (see eq. 2.28). Empirically they have found that diffusion constants of the two components change depending on the lipid composition. These curves can also be examined by an anomalous diffusion fit, where the mean square displacement is not linear with time, but depends on $t^{\alpha}$, with $0<\alpha<1$ (see eq. 2.29). The autocorrelation curves of fig. 4.6 at $T=303.0 \mathrm{~K}$ were described by both means. The following results were obtained:

1. Two component fit (data not shown):

$$
\begin{array}{lll}
\tau_{1}=113 \text { MC time }, \tau_{2}=1497 \text { MC time, } & \text { ra }=0.21, & \text { ratio }=1: 1 \\
\tau_{1}=187 \text { MC time }, \tau_{2}=2648 \text { MC time }, & \text { ra }=0.29, & \text { ratio }=1: 100 \\
\tau_{1}=346 \text { MC time, } \tau_{2}=2999 \text { MC time }, & \text { ra }=0.48, & \text { ratio }=1: 1000 \\
\tau_{1}=665 \text { MC time, } \tau_{2}=22438 M C \text { time }, & \text { ra }=0.65, & \text { ratio }=1: \infty
\end{array}
$$

2. Anomalous fit (red curves in fig. 4.6):

$$
\begin{array}{lll}
\tau=296 \text { MC time }, & \alpha=0.83, & \text { ratio }=1: 1 \\
\tau=279 \text { MC time }, & \alpha=0.79, & \text { ratio }=1: 100 \\
\tau=190 \text { MC time }, & \alpha=0.73, & \text { ratio }=1: 1000 \\
\tau=117 \text { MC time }, & \alpha=0.63, & \text { ratio }=1: \infty,
\end{array}
$$


with ratio always referring to the ratio of state changes to diffusion steps and $r a$ is the ratio of the fast to slow diffusion component.

One sees that the phenomenological constants which are a result of the fit procedures depend on the diffusion and relaxation timescales. Therefore, one can conclude that the fitting procedures do not give any information about the underlying physical processes. This finding is further discussed in sec. 5.1.

\section{Effect of Interaction Parameters}

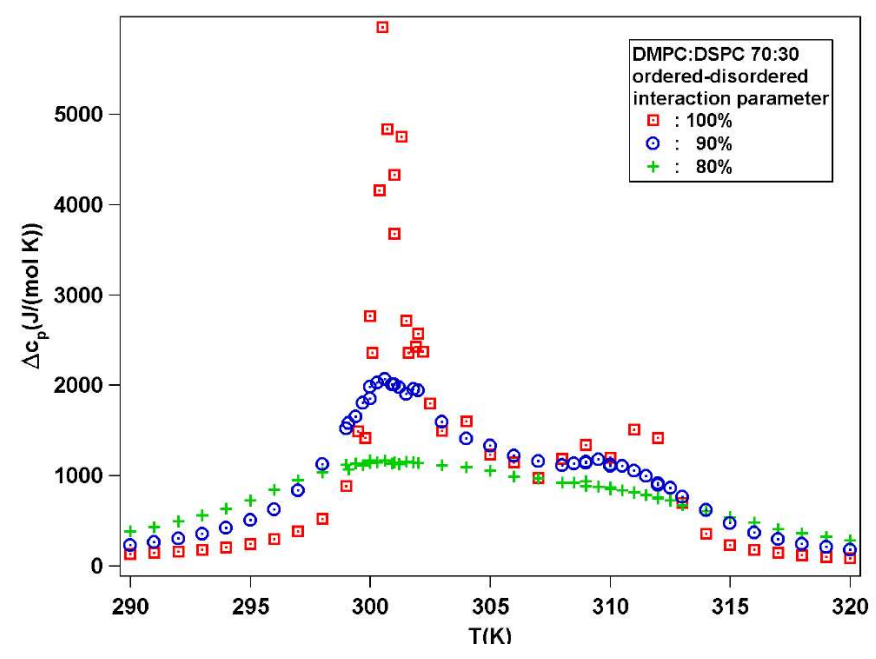

Figure 4.7: Heat capacity profiles of DMPC:DSPC 70:30 with changed interaction parameters. Lowering the interaction parameters between ordered and disordered chains to $90 \%$ or $80 \%$ of their original values (see table 3.1) affects the melting profile. The profile broadens with lower interaction parameters and therefore the maximum heat capacity value decreases. Higher interaction parameters mean that contacts between ordered and disordered chains are less favorable than for lower parameters.

In the following another aspect is studied. In section 4.3 it is shown that small molecules like alcohols or neurotransmitters broaden the excess heat capacity profile and shift the profile to lower temperatures. Formally speaking, broadening means that the interaction parameters in our model are reduced. The model and the evaluation by means of MC simulations allows one to study the effect of a reduction of ordered-disordered interaction parameters without a shift of the profile to lower temperatures. In fig. 4.7 three calculated heat capacity profiles of a DMPC:DSPC 70:30 mixture with different ordered-disordered interaction parameters are displayed. One reflects the case of using the interaction parameter values needed for the description of the phase diagram of the DMPC:DSPC membrane system (see table 3.1). In the simulations of the other profiles ordered-disordered interaction parameters were reduced to $90 \%$ or $80 \%$ of their actual values. The curves are broadened, but not shifted. The decrease of interaction parameters and therewith the broadening of the $\Delta c_{p}$-profiles also means that the lateral membrane order changes (see figs. 4.30 and 4.31 in sec. 4.5). Simulations of diffusion processes with reduced interaction parameters were performed to study the influence of the structural order and changes of fluctuation properties (see sec. 4.5) on diffusion processes, as it might be influenced by alcohols or neurotransmitters. 

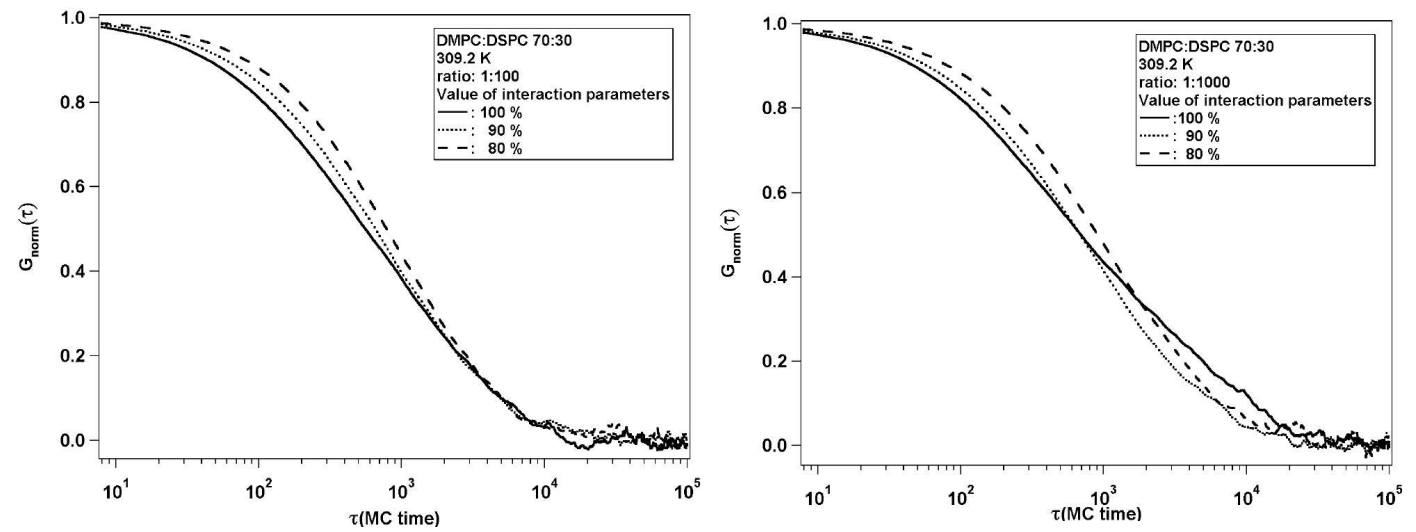

Figure 4.8: FCS simulations with different interaction parameters. Small molecules can reduce the interactions at domain interfaces. Interaction parameters decrease, and this results in a change of matrix configuration and fluctuations (see sec. 4.5). The diffusion behavior is changed too. A reduction in interaction parameters can lead to a normal diffusion behavior. Here, simulation results of a DMPC:DSPC $70: 30$ mixture at 309.2 K are shown. In the two cases the ratio between state changes and diffusion steps is different $(1: 100$ (left) and 1 : 1000 (right)).

In fig. 4.8 autocorrelation profiles of fluorescence intensity traces from simulations of a DMPC:DSPC $70: 30$ at $309.7 K$ are presented. Ordered-disordered interaction parameters are set to $100 \%, 90 \%$ and $80 \%$ of the originally determined values. In the left and right panel of fig. 4.8 simulations were performed with a ratio between state changes and diffusion steps of $1: 100$ and $1: 1000$, respectively. In the case of a ratio of $1: 100$, diffusion deviates from normal diffusion when ordered-disordered interaction parameters are set to $100 \%$ and $90 \%$. The autocorrelation profile simulated with $80 \%$ ordered-disordered interaction parameters can be described by normal diffusion (fits not shown). As described above the ratios between state changes and diffusion steps influence the autocorrelation profiles. The slower the state changes in comparison to diffusion steps the higher becomes the $\alpha$-value describing the anomalous diffusion behavior. Therefore, in the cases with changed interaction parameters also the state change to diffusion ratio affects the diffusion behavior. With a ratio of $1: 100$, diffusion is normal on the length scale of the focus at parameters reduced to $80 \%$. This is not true any more at a ratio of $1: 1000$, where all profiles can only be described by anomalous diffusion or two component diffusion fits. Just concentrating on anomalous diffusion fits, one finds that $\alpha$-values are $\alpha_{100 \%}=0.738, \alpha_{90 \%}=0.939$ and $\alpha_{80 \%}=0.965$. The deviation from normal diffusion behavior is bigger in the case of high interaction parameters. This is true for all conducted simulations at DMPC:DSPC ratios and temperatures as of fig. 4.2. Decreasing ordered-disordered interaction parameters can speed up or slow down diffusion.

\section{Summary}

Fluorescence intensity autocorrelation profiles can be predicted by our MC simulations. Diffusion processes in binary lipid membranes of DMPC and DSPC in the domain coexistence regime display a behavior deviating from normal diffusion. Diffusion properties are influenced by the time scales of diffusion steps and fluctuations in chain state. This information is hidden in measured fluorescence intensity autocorrelation profiles. The reduction of ordered- 
disordered interaction parameters influences the melting behavior and therein the diffusion behavior.

\subsection{Melting Transitions of Artificial Membranes}

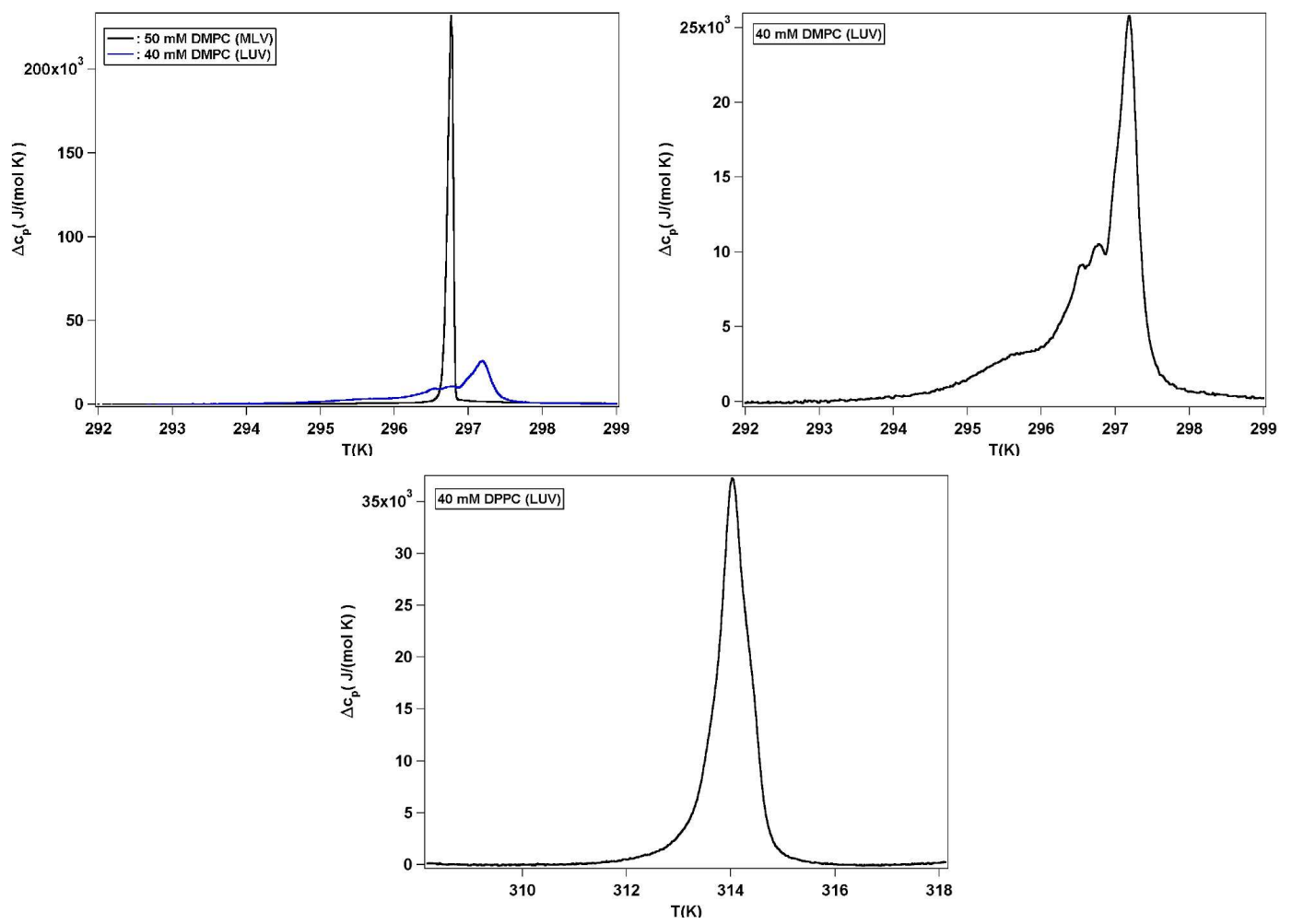

Figure 4.9: Heat capacity profiles of one component lipid membranes. The melting temperature and the profile's shape depend on chain length and the vesicle type. MLV vesicles show a higher cooperativity than LUVs (top panel left and right, respectively). The melting temperature differs as well. Longer chain length results in higher melting temperatures (upper panel right and lower panel).

As explained in the introductory part of this work (see ch. 1) melting processes in lipid membranes depend on different parameters like chain length, head group, $\mathrm{pH}$, pressure, ion concentration and vesicle type. In this work uncharged phospholipids are used whose melting profile is hardly affected by ion concentration or $\mathrm{pH}$. As shown below they, however, depend on lipid chain length, size of the lipid vesicles and the addition of extra molecules like alcohols, neurotransmitters or peptides. In the following, data is presented which shows excess heat capacity profiles of different phospholipid membrane systems. All measurements were done with lipid vesicles formed in a $10 \mathrm{mM}$ Hepes buffer with $1 \mathrm{mM}$ EDTA at $\mathrm{pH}$ 7. All scans were done with the VP-DSC, with the exception of the DMPC:DSPC plus 1-octanol system. Melting profiles of the latter system were conducted with the MSC. All curves shown, with the exception of the DMPC/Gramicidin A system, were measured at scan rates of $5 \frac{K}{h}$ with increasing temperatures with time. In the case of the DMPC/Gramicidin A system the scan rate was $1 \frac{K}{h}$. 

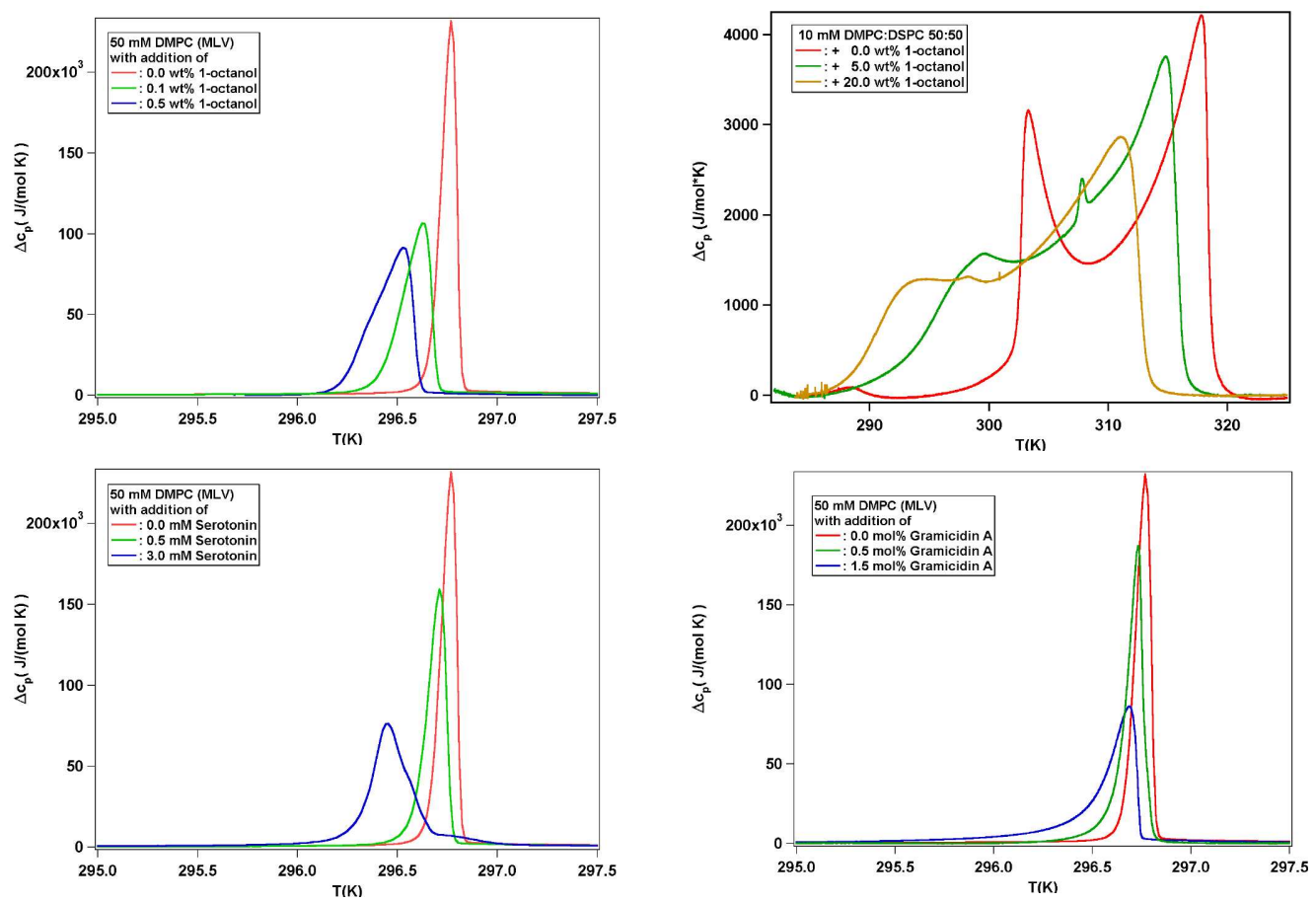

Figure 4.10: Heat capacity profiles of phospholipid membranes (MLV) with small molecules or peptides. DSC scans were performed on suspensions of DMPC MLVs with addition of different concentrations of 1octanol (top panel, left), serotonin (bottom panel, left) and gramicidin A (bottom panel, right). Another series of measurements was done with equimolar mixtures of DMPC:DSPC MLVs and addition of various concentrations of 1-octanol (top panel, right). The addition of 1-octanol, serotonin and gramicidin A leads to a broadening of the profiles and a shift of the transition regime to lower temperatures.

In fig. 4.9 melting profiles of pure lipid membranes are depicted. The first two graphs (upper panel left and right) display curves of pure DMPC membranes. DMPC is a phospholipid with 14 carbon atoms in each of its chains. It can be seen that the DMPC MLV system shows a higher cooperativity than the LUVs. In the latter case the profile is broadened and the heat capacity maximum is shifted to higher temperatures. Comparing heat capacity curves of LUVs of DMPC and DPPC (16 carbon atoms per chain) it is seen that the DPPC membranes melt at higher temperatures.

Addition of the alcohol 1-octanol broadens melting profiles of one component DMPC MLVs and of equimolar DMPC:DSPC MLVs and shifts them to lower temperatures (fig. 4.10 top panel left and right). It is important to note that the amount of 1-octanol in the buffer and the membranes is given with respect to the lipid weight. Alcohol molecules partition into water and lipid membranes. Thus, one has to know the partition coefficient to calculate the molar amount of 1-octanol partitioned into the lipid membrane. This is discussed in more detail elsewhere (see sec. 5.1.3). The neurotransmitter serotonin and the antibiotic peptide gramicidin A also shift the profiles to lower temperatures and broaden them (fig. 4.10 bottom panel left and right). In fig. 4.10 the concentration of serotonin is given as it was dissolved in the buffer solution. The molar ratio of gramicidin A is the one in the lipid membrane.

In the following section results from relaxation time measurements on the different systems 
(with exception of the two component lipid system) are shown. The addition of small molecules or peptides influences heat capacity profiles. The underlying question is whether measured relaxation processes are still related to heat capacity as proposed by Grabitz et al. (2002).

\section{Summary}

Anesthetics, neurotransmitters and antibiotics broaden melting profiles and shift them to lower temperatures.

\subsection{Relaxation Processes of Artificial Membranes}

Relaxation experiments with artificial lipid membranes were performed using pressure perturbation calorimetry. Two methods are used for the evaluation of relaxation processes. One in which we monitor the response of our VP-DSC calorimeter after a rapid pressure change with the assumption of constant temperature. A second method measures a temperature change directly in the sample. (Grabitz et al., 2002) applied already successfully the method using the response of the calorimeter. Evaluating the temperature behavior after a pressure jump has been developed in the context of this thesis in order to increase the time resolution of our relaxation time experiments. In the next two sections the principal findings and problems of the two methods are described and compared. After that results of relaxation measurements on different systems are presented.

\subsubsection{Pure DMPC Membrane: Method I}

The calorimeter response after a negative or positive pressure jump ( $n p j$ or $p p j$ ) gives information about the relaxation process of the lipid system. The calorimeter response is assumed to be the result of a convolution of the instrument response and the lipid signal. One of the simplest systems to study is a solution of multilamellar DMPC vesicles. In the left panel of fig. 4.11 an excess heat capacity profile is compared to measured relaxation times. Experiments were performed on a $50 \mathrm{mM}$ DMPC suspension (MLV; 14 carbon atoms per chain). The shown $\Delta c_{p}$-curve was measured with the VP-DSC at a scan rate of $5 \frac{K}{h}$. The solution was filled into the pressure cell whose volume is not exactly known. This means that no absolute values of heat capacity can be obtained directly, since for that the lipid concentration, the cell volume, the scan rate and the calorimeter signal are needed. However, measurements on a $10 \mathrm{mM}$ solution directly in the calorimeter cell which has a defined volume allowed us to determine the transition enthalpy ${ }^{3}$ in units of $\frac{J}{m o l}$. This does not depend on the lipid concentration and therefore, the curve from a solution with a concentration of $50 \mathrm{mM}$ should yield the same transition enthalpy. This allows the recalculation of the measured profile of lipid membranes in the pressure cell to absolute values. In this case the transition enthalpy was determined to be $21.5 \frac{\mathrm{kJ}}{\mathrm{mol}}$.

Pressure perturbation experiments were done by changing pressure with $\Delta p= \pm 40 \mathrm{bar}$. Three jumps at the same temperature were evaluated and averaged. Results from negative pressure jumps (npjs; circles) and positive pressure jumps (ppjs; squares) are drawn together. Relaxation times are as long as $31.0 \mathrm{~s}$ and standard deviations are usually not

\footnotetext{
${ }^{3}$ The transition enthalpy is the area below the heat capacity profile.
} 

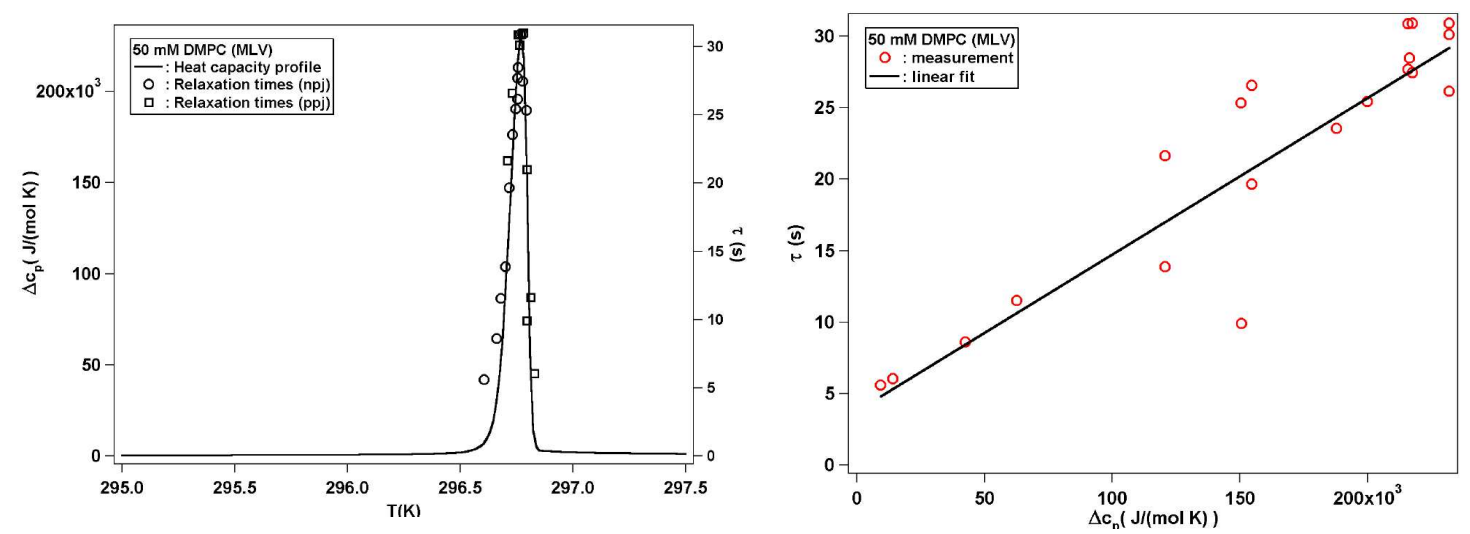

Figure 4.11: Heat capacity and relaxation times of DMPC (MLV). (left) An excess heat capacity profile (solid curve; left axis) and relaxation times (markers; right axis) of a $50 \mathrm{mM}$ DMPC (MLV) solution are shown. The maximum of the relaxation times is at a temperature where the heat capacity displays a maximum too. The relaxation times were measured with npjs (circles) and ppjs (squares). The temperatures of the ppjs needed to be corrected to lower temperatures, since the melting temperature depends on pressure and becomes higher with increasing pressure. (right) Relaxation times are plotted against heat capacity. The measured data (red, open circles) can be fitted by a linear function (black, solid curve). The fit does not intersect the origin. The reason for this is believed to originate from the experimental limitations.

bigger than $0.5 \mathrm{~K}$. A comparison of the heat capacity profile and the relaxation profiles reveals (see the left panel of fig. 4.11) that relaxation processes slow down in the proximity of the melting regime. The maximum relaxation time is found at a temperature where also the heat capacity displays a maximum. Plotting relaxation times against respective heat capacities shows that relaxation time and heat capacity display a linear relationship (right panel of fig. 4.11). The corresponding linear fit does not intersect the origin. An intersection with the origin was, however, expected from the theoretical considerations and conclusions of Grabitz et al. (2002). This were that relaxation time and heat capacity are related to each other by: $\tau=\frac{T^{2}}{L} \Delta c_{p}$, where $\tau$ is the relaxation time, $T$ the temperature, $L$ the phenomenological coefficient and $\Delta c_{p}$ the heat capacity (see also sec. 3.2). Later in the text we argue that this results rather from experimental limitations than from the physics of the system. Further, we want to determine the phenomenological constant of the investigated system. From the heat capacity value at the transition midpoint, $\Delta c_{p}^{m}=2.32 \frac{k J}{m o l K}$, the transition midpoint, $T_{m}=296.77 \mathrm{~K}$, and the relaxation time at the transition midpoint, $\tau_{m}=31.0 \mathrm{~s}$, one calculates a phenomenological coefficient of $6.6 \cdot 10^{8} \frac{\mathrm{JK}}{\mathrm{mols}}$.

\section{Compensation of Heat Release and Absorption}

Doing npjs one jumps from the "so" phase into the coexistence region so the calorimeter needs to heat the sample. In the other case of performing ppjs into the coexistence regime it needs to cool. The heating and cooling needs to compensate the heat absorption or release of the lipids. If, however, one jumps through the whole transition or through a major part of it the calorimeter fails to compensate the heat release or absorption. This problem is more pronounced in the case of ppjs since cooling of the cells is slower. This means that the cell's 
temperature cannot be kept constant. In a case like this an analysis of the calorimeter response is not possible. The high cooperativity of the lipid membrane transition requires a good control of temperature so that the point in phase state is not shifted during the measurement. In fig. 4.12 two situations are presented. One in which heat could be compensated (left) with a temperature fluctuating around an average and another one where the temperature increased after a ppj. Even though this problem is, because of the slow cooling, more common for ppjs it also occurs in the case of npjs. A high lipid concentration, of course, influences the likelihood of the occurrence of a failure in compensation.
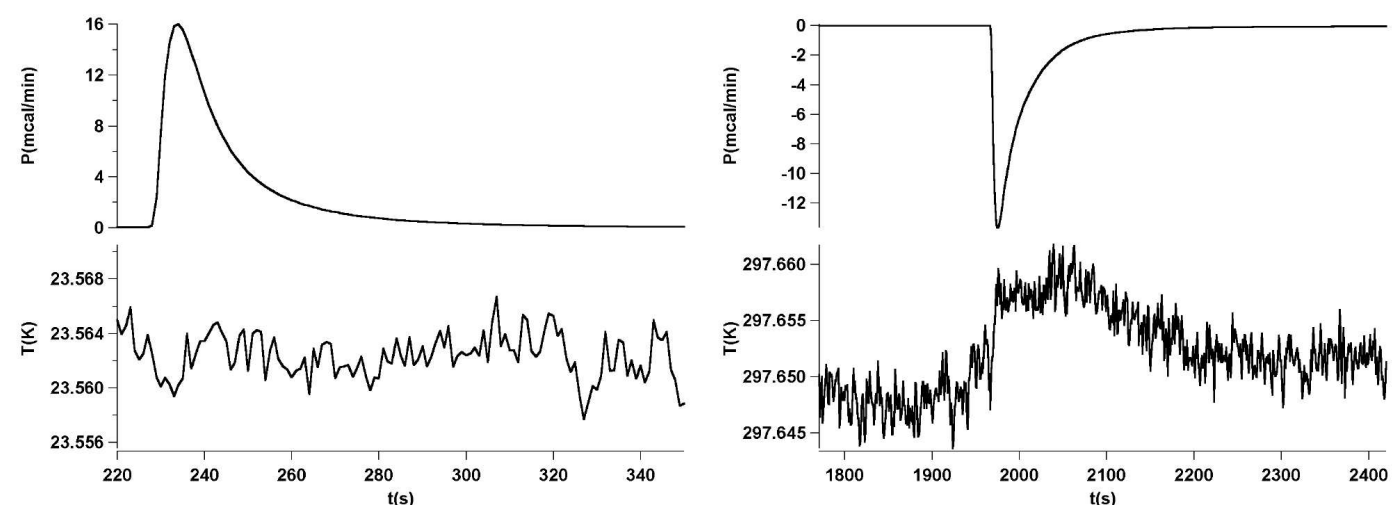

Figure 4.12: Temperature course after a pressure jump (method I). (left) A typical calorimeter response (upper graph) after a npj and the temperature development (lower graph) are shown. (right) The answer of the calorimeter and the temperature versus time curve after a $p p j$ are displayed. One should note the different sizes of the two temperature axes of the left and right graph.

For npjs one usually gets problems at temperatures corresponding to the upper temperature limit of the transition. For ppjs it is at the lower temperature limit. Therefore, results from npjs and ppjs are plotted together. The results complement each other. In fig. 4.11 results from npjs are shown using circles, whereas the ones from ppjs are indicated by squares. Since the transition under pressure happens at higher temperatures $\left(\Delta T_{m} \approx 0.95 \mathrm{~K}\right.$ at $\Delta p=40 \mathrm{bar}$ ) the latter results have to be temperature corrected. Usually, one expects a correction of $0.95 \mathrm{~K}$ to lower temperatures, but the temperature scale had to be shifted by $-0.90 \mathrm{~K}$. Unexpectedly, also, the relaxation times from npjs have to be corrected by shifting the relaxation times by $-0.01 K$. Already Grabitz et al. (2002), who worked with the same calorimeter and setup, reported a shift of their data of $-0.04 \mathrm{~K}$. They claimed that it was due to differences in the temperature calibration of the isothermal and scanning mode of the VP-DSC. This, however, cannot explain why values from ppjs have to be corrected by $-0.90 \mathrm{~K}$ and not the expected value of $-0.96 \mathrm{~K}$. A possible physical explanation lies in another effect which is known as hysteresis.

\section{Hysteresis Effects}

Fig. 4.13 displays heat capacity profiles of the same lipid suspension as in fig. 4.11 obtained with different scan rates and scan directions. Doing scans from lower to higher temperatures with faster scan rates result in melting at higher temperatures and broader profiles than the ones measured at slower scan rates. Scanning from high to low temperatures (down scan) 

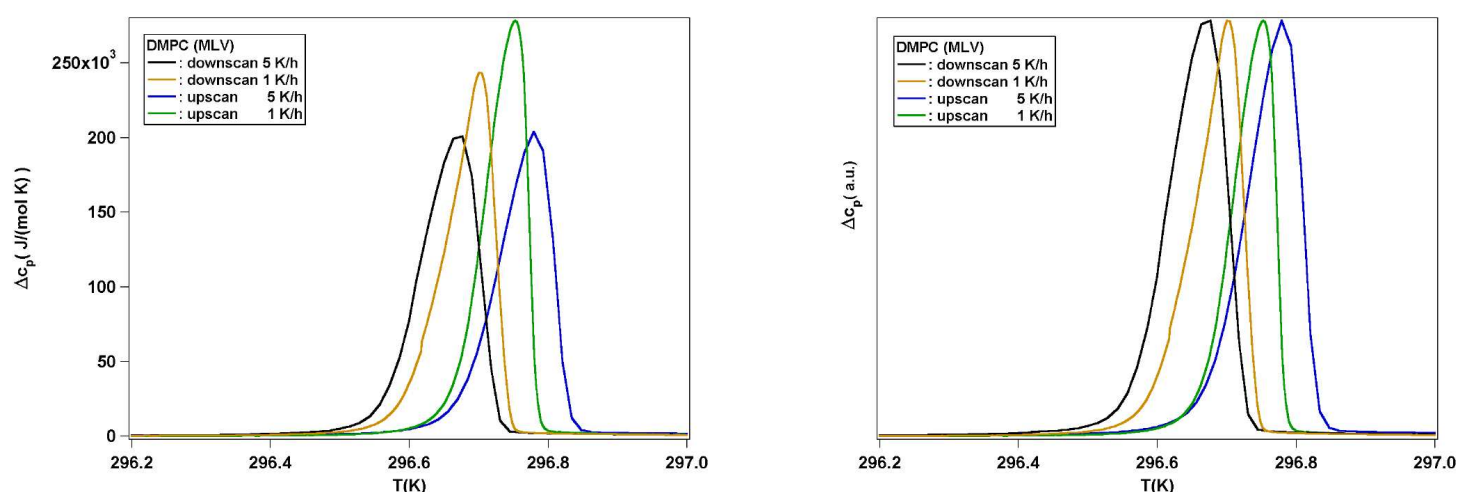

Figure 4.13: Hysteresis effects in a DMPC Suspension. Different DSC-scans with different scan rates and with different scan directions result in unequal $\Delta c_{p}$-curves. The profiles display different degrees of broadening and also different transition midpoints. In one of the graphs the absolute heat capacity values are plotted (left), while in the other one the normalized profiles are shown (right).

leads to melting at lower temperatures than in the case of scanning the opposite direction (up scan). In the case of down scans higher scan rates lead to melting at lower temperatures. The maximum chosen scan rate was $5 \frac{K}{h}$. An even faster scan would shift the melting temperature to even higher (up scan) or lower (down scan) temperatures.

Measurements showed that in the case of a profile measured with $10 \frac{K}{h}$, the relaxation times did not need shifting. This suggests that a sudden change in pressure should be compared to a profile measured at high scan rate. Because of the small half width of the melting profiles, however, it does not make sense to present curves measured at a scan rate of $10 \frac{K}{h}$. If one compares the relaxation times to heat capacity profiles measured at slower scan rates a shift of the relaxation data becomes necessary. This shift corresponds to the fact that the heat capacity maxima of profiles measured with different scan rates occur at varying temperatures. Still, the general message that relaxation times and heat capacity are linearly related stays true.

Performing ppjs one jumps from a "ld" environment into the phase coexistence regime. Therefore, it seems reasonable that one should consider heat capacity profiles measured with downwards scanning. Since measured profiles from down scans and up scans at the same scan rate differ, it is not surprising that the relaxation time values have to be shifted by another value instead of the expected one of $-0.96 \mathrm{~K}$. The shift in melting temperature between a down scan and an up scan, both measured at a scan rate of $5 \frac{K}{h}$, is about $0.11 K$. This means that the necessary shift of $-0.90 K$ differs from what one would expect $(-0.84 K)$ considering the down scan at $5 \frac{K}{h}$.

\section{Transition Enthalpy and Heat Release/Absorption}

A last aspect of the evaluation of relaxation processes with method I concerns the total heat release or absorption of the calorimeter after a pressure jump. This should be proportional to the transition enthalpy. In fig. 4.14 the solid curve shows the transition enthalpy dependence on temperature. It is the integral of the excess heat capacity. Plotting also the total area of the calorimeter responses at different temperatures verifies that it is proportional to the 


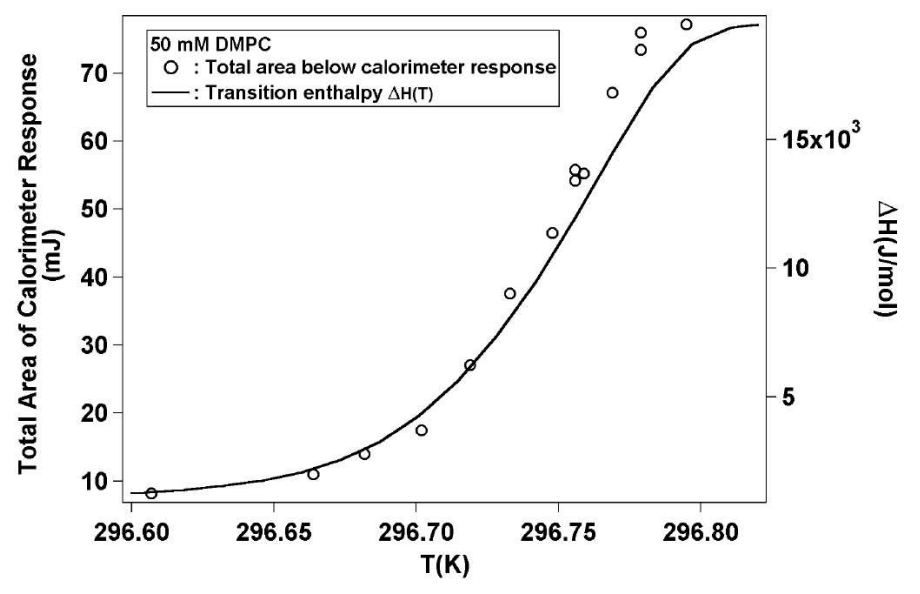

Figure 4.14: Transition enthalpy and total area of calorimeter response versus temperature. It is verified that the area of the calorimeter response after npjs (markers) and the transition enthalpy (solid curve) are proportional.

transition enthalpy.

Before further results obtained with this method are presented, the next section introduces method II, where the temperature signal after a pressure jump is monitored. Both ways of analysis should yield the same relaxation behavior.

\subsubsection{Pure DMPC: Method II}

Using method I it is assumed that the temperature in the lipid solution stays constant after a pressure jump. This is, however, just an idealized situation and in reality the temperature changes. The temperature sensors of the calorimeter are not able to monitor this, but thermocouples which are directly led into the pressure cell capillaries can be used for this purpose. Again, we assume that a convolution of the instrument response with the lipid signal describes the measured temperature course.

\section{Multilamellar Vesicles}

Experiments on a suspension of multilamellar DMPC vesicles can be compared to measurements from method I as shown in fig. 4.11. In fig. 4.15 a $\Delta c_{p}$-profile measured for a $40 \mathrm{mM}$ DMPC in a $10 \mathrm{mM}$ Hepes buffer with $1 \mathrm{mM}$ EDTA at $p H 7$ at a scan rate of $5 \frac{K}{h}$ is presented. The heat capacity values were corrected so that the area of the curve equals $21.5 \frac{\mathrm{kJ}}{\mathrm{mol}}$. Markers in the same graph display relaxation times as obtained after ppjs of +15 bar. The shown values are averages from two jumps at the same temperature. They are shifted so that they fit onto the heat capacity curve at atmospheric pressure $(-0.33 \mathrm{~K})$. It is again observed that relaxation times become longer at temperatures where the heat capacity displays anomalies. The longest relaxation time is found at a temperature were the heat capacity is the highest. Where the maximum relaxation time was determined as $\tau_{m}=31.0 \mathrm{~s}$ with method I, it was measured as $\tau_{m}=25.2 \mathrm{~s}$ with method II. The sample preparations used were different. Performing DSC scans of the two samples it was found that 


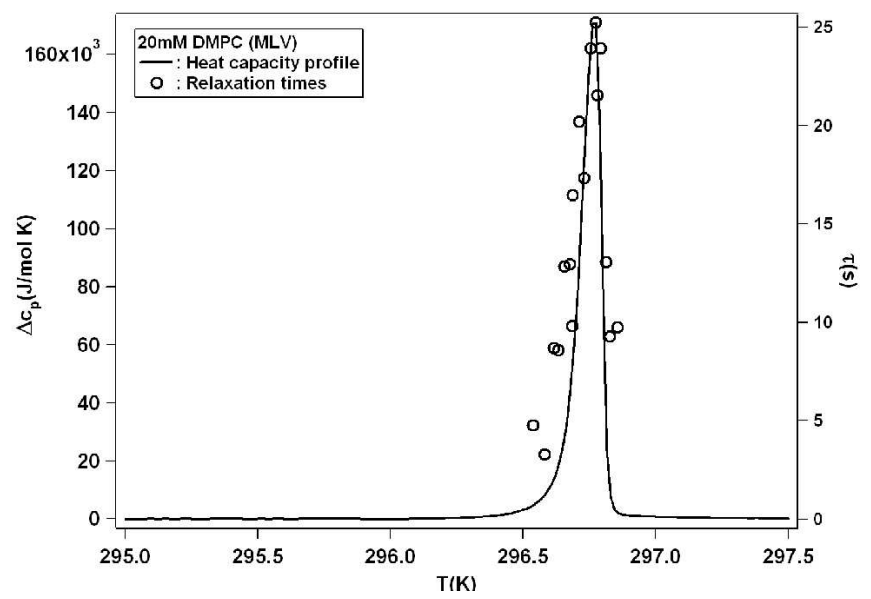

Figure 4.15: Relaxation times of a multilamellar DMPC solution evaluated with method II. Shown are a heat capacity profile (solid curve) and relaxation times (open circles) of multilamellar DMPC vesicles. The relaxation times were obtained by evaluating the temperature development of the sample solution.

the two profiles differed. The heat capacity curve of the sample which was used during the evaluation with method II was broader and the maximum heat capacity value was decreased in comparison to the profile of the sample which was used during the evaluation with method I. The transition temperature, however, was the same in both measurements. One finds a phenomenological constant of $L=6.8 \cdot 10^{8} \frac{\mathrm{JK}}{\mathrm{mols}}$ from the measurements of method II. Besides a small deviation it equals the one obtained with method I $\left(L=6.6 \cdot 10^{8} \frac{\mathrm{JK}}{\mathrm{mols}}\right)$. The phenomenological constants, obtained from both methods, should be the same rather than the absolute relaxation times. Therefore, it can be concluded that the analysis of pressure perturbation experiments with method I or method II give the same relaxation behavior. This is further discussed in sec. 5.1.4.

\section{Large Unilamellar Vesicles}

The advantage of method II in comparison to method I is its time resolution. Method I has a time resolution of about $3-4 s$. The direct measurement of the temperature course allows one to measure relaxation times with a resolution of less than $500 \mathrm{~ms}$. An example can be seen in fig. 4.16. Pressure jump experiments on a $40 \mathrm{mM}$ extruded DMPC solution with changing pressure of $+40 \mathrm{bar}$ were performed. At each temperature two experiments were used to find an average relaxation time. The longest relaxation process in DMPC LUVs is much faster than the longest one found in DMPC MLVs. A linear relationship between the excess heat capacity and relaxation time is again present. The maximum relaxation time was found to be $\tau_{m}=1.5 \mathrm{~s}$. We estimate a phenomenological constant of $L=15.7 \cdot 10^{8} \frac{\mathrm{JK}}{\mathrm{mols}}$. This phenomenological coefficient is more than a factor of two bigger than of DMPC multilamellar vesicles.

\section{Problems of Method II}

A few problems related to this method are present. One problem is due to the weakness 


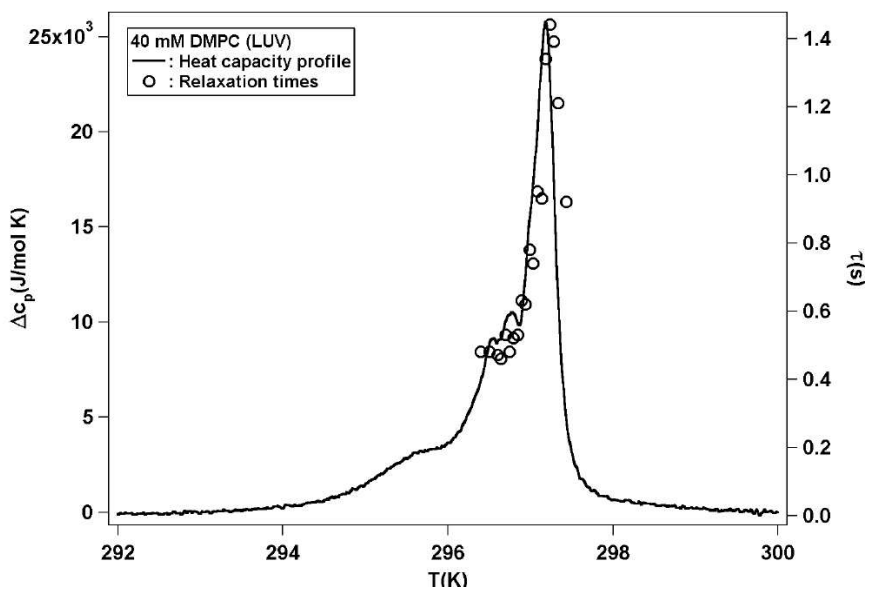

Figure 4.16: Relaxation times of a LUV DMPC solution. Relaxation times (open circles) were determined on experiments with a suspension of $40 \mathrm{mM}$ DMPC (LUV). The corresponding heat capacity profile (solid curve) is shown too. LUVs of DMPC are less cooperative than DMPC MLVs. The maximum relaxation time $\left(\tau_{m}=1.5 s\right)$ is much faster than the one of DMPC MLVs $\left(\tau_{m} \approx 25 s\right.$ or even slower $)$.

of the signal originating from the lipid membranes (further on called the lipid signal) at the upper temperature limit of the heat capacity curve. Performing ppjs means that at higher temperatures the change in enthalpy after a pressure jump is smaller than at lower temperatures. The weaker strength of the lipid signal at higher temperatures complicates the resolution of the lipid signal. The determination of relaxation times is difficult and results in a lack of data at higher temperatures. Since the time resolution of npjs is not high enough one cannot use measurements with pressure release for complementing the kinetic measurements with ppjs.

Another problem might occur because of the width of the melting transition. In experiments of lipid systems with high cooperativity an additional pressure of 40 bar shifts the profile away from the one at atmospheric pressure. The two curves do not intersect. In cases of less cooperative processes this, however, is not the case (data not shown) and profiles overlap. If a temperature is chosen at which the two profiles overlap, one jumps from a point in the coexistence regime into another one in the coexistence regime by increasing pressure. This leads to a smaller heat release than in the case of jumping from a pure "ld" membrane into the coexistence regime. This means that with further lowering of temperature the heat release decreases instead of increasing. The problem is, however, less pronounced than at higher temperatures. This means that the added pressure should be chosen to be as big as possible. Varying pressure does not change relaxation times (data not shown). With higher pressure changes the perturbation signal increases as well. A pressure change of 40 bar when doing experiments on suspensions of LUVs was chosen, which was sufficient in our experiments.

Of course, the lipid signal also depends on the lipid concentration. If the concentration is higher the lipid contribution grows. At some temperatures this can lead to a total signal which shows too big a temperature change and the relaxation process cannot be evaluated. This is because the point in the coexistence regime changes during the measurement and the temperature course would reflect several relaxation properties. This problem is more pronounced in studies on MLVs than on LUVs. The cooperativity is much higher and already 
minor temperature changes can change the phase state. Parameters like concentration and pressure change have to be carefully chosen.

\section{Summary}

In the previous two sections two methods have been introduced which allow one to study relaxation processes in artificial lipid membranes. First experimental results of simple one component systems (MLV and LUV) were described. Heat capacity and relaxation time are found to be proportional to each other. Further, these two methods were used for studying the relaxation behavior of different systems with addition of anesthetics, or neurotransmitters or antibiotics. The type of vesicle system (MLV or LUV) was varied too. The question of interest was whether the linear relationship between relaxation times and heat capacity is valid for a diverse of systems.

\subsubsection{Addition of Small Molecules or Peptides and Type of Vesicle}

In the previous two sections it is described that one can determine relaxation times of lipid suspensions after a pressure perturbation using the response of a VP-DSC calorimeter or evaluating the directly measured temperature behavior. First measurements on pure lipid membrane systems display a linear relation between relaxation times and heat capacity, as theoretically predicted (Grabitz et al., 2002). Since the transition of LUVs shows a lower cooperativity than the one of MLVs, relaxation times at a temperature of maximum heat capacity are much faster in LUV systems.

This finding raises new questions about whether this linear relation is still valid for systems with additional molecules in the lipid bilayer. Further experiments were based on the addition of molecules which display a functional role in biological membranes. These molecules are the anesthetics 1-octanol and halothane, the neurotransmitter serotonin and the antibiotic gramicidin A. As described in sec. 4.3 these molecules influence the transition behavior of the lipid membrane. All of them shift the profile to lower temperatures and broaden the profile ${ }^{4}$.

Pressure jump experiments with different systems were performed changing pressure with \pm 40 bar. At each temperature three pressure jumps were evaluated with method I and the obtained relaxation times were averaged. In the graphs (fig. 4.17 and 4.18) the displayed heat capacities were measured with a scan rate of $5 \frac{K}{h}$, where the transition enthalpy is standardized to $21.5 \frac{\mathrm{kJ}}{\mathrm{mol}}$. Relaxation times were shifted by $-0.01 \mathrm{~K}$ for $n p j s$ and by $-0.90 \mathrm{~K}$ for ppjs. It is, however, not indicated which points come from npjs and which from ppjs.

\section{Influence of Anesthetics}

In order to verify the influence of anesthetics, different amounts of 1-octanol given in percentages of the lipid weight were given into a $50 \mathrm{mM}$ suspension of multilamellar DMPC vesicles (see fig. 4.17). Addition of the anesthetic 1-octanol broadens the transition profile and shifts it to lower temperatures. The higher the concentration the bigger the effect. It is also found that the maximum heat capacity decreases with increasing 1-octanol concentrations. This also affects the relaxation process. The region of increased relaxation times is as much shifted as is the heat capacity profile. The longest measured relaxation process becomes

\footnotetext{
${ }^{4}$ No data in the case of halothane is shown in sec. 4.3. The conclusion is the same as in the case of 1-octanol.
} 

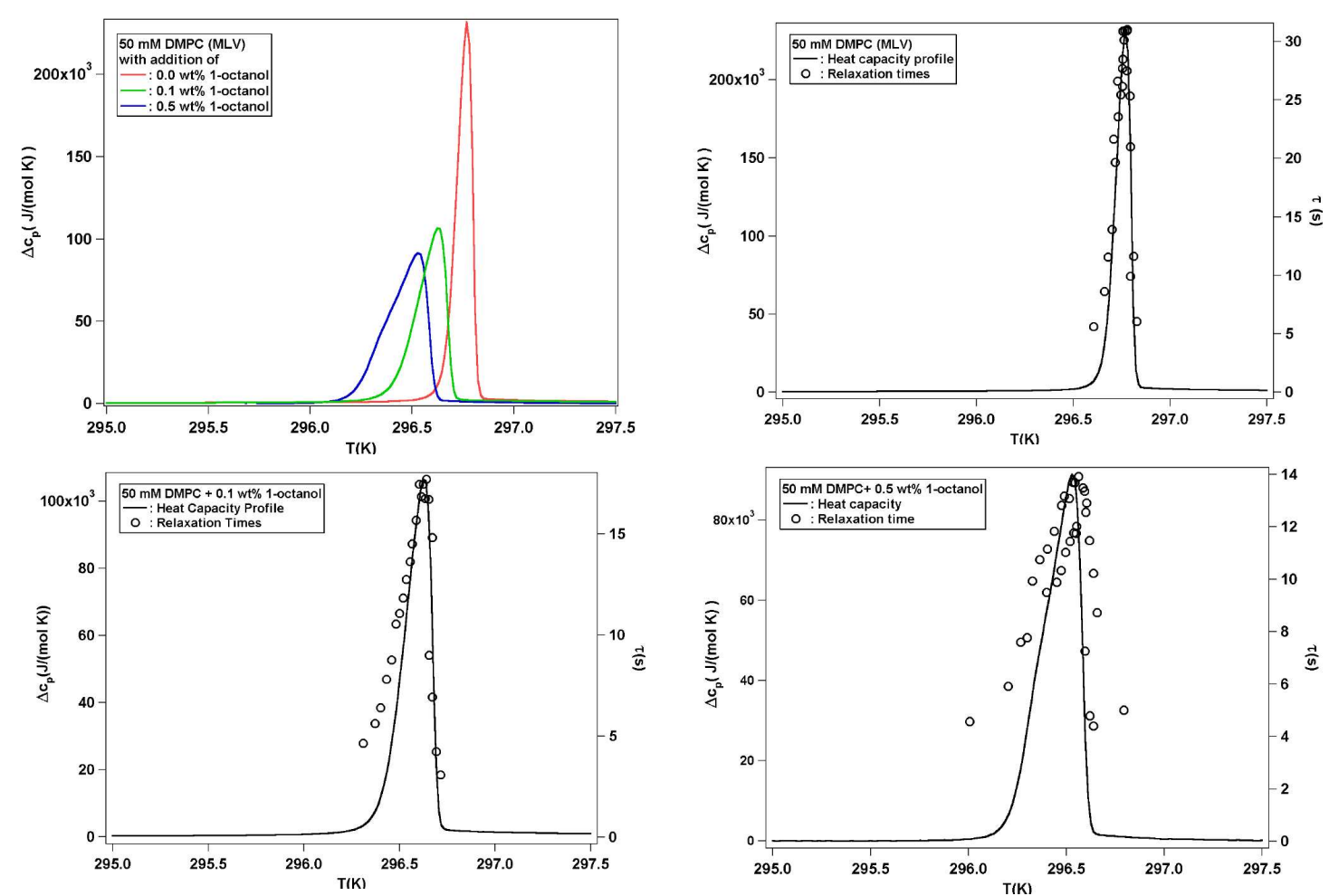

Figure 4.17: Relaxation times of a DMPC solution (MLV) with addition of the anesthetic 1-octanol. In the different panels heat capacity profiles (solid curves) and relaxation times (open circles) are shown from measurements with addition of different concentrations of 1-octanol: (top panel, right) pure DMPC Membrane, (bottom panel, left) DMPC membrane plus $0.1 w t \%$ 1-octanol and (bottom panel, right) plus $0.5 w t \%$ 1-octanol. One should note that 1-octanol shifts the heat capacity profile to lower temperatures and broadens it (top panel, left). Therein, the maximum heat capacity value becomes smaller. Relaxation times are also affected. The maximum relaxation time decreases with increasing anesthetic concentration and the regime of slowed down relaxation processes is shifted to lower temperatures too. Note the different scaling of the heat capacity and relaxation time axes of the graphs displaying relaxation times and the respective $\Delta c_{p}$-profile.

faster the more the maximum heat capacity value decreases. This finding supports that heat capacity and relaxation times are proportional also after the addition of 1-octanol. Depending on the degree of broadening the maximum relaxation time decreases. In the case of adding $0.1 w t \% 1$-octanol one finds a relaxation time of $17.7 s$ and when adding $0.5 w t \%$ 1-octanol we determined $\tau_{m}=14.8 \mathrm{~s}$ at the transition midpoint. Calculating the phenomenological constants one finds from both measurements $L=6.3 \cdot 10^{8} \frac{\mathrm{JK}}{\mathrm{mols}}$. These values lie in the same order as determined from experiments on the DMPC MLVs $\left(L=6.6 \cdot 10^{8} \frac{\mathrm{JK}}{\mathrm{mol} \mathrm{s}}\right.$ and $L=6.8 \cdot 10^{8} \frac{\mathrm{JK}}{\mathrm{mol} \mathrm{s}}$ ). Another measurement with addition of $0.1 w t \% 1$-octanol (different preparation) resulted in a phenomenological constant of $L=7.2 \cdot 10^{8} \frac{\mathrm{JK}}{\mathrm{mol} \mathrm{s}}$, which is higher than the one from the measurement with the other preparation. This discrepancy can be explained with experimental problems. This is that DSC scans of the same sample on successive days can result in heat capacity profiles which show small differences. Often it is found that profiles broaden with time. A broadening automatically results in a decrease of the maximum heat capacity and as seen faster relaxation processes. Taking a heat capacity 
profile from an earlier day as a reference results in a bigger phenomenological constant. In the latter series of measurements a broadening of the heat capacity curves was recorded. However, no scan after finishing the relaxation measurements was done. A further broadening might explain the small deviation of the two phenomenological constants. Another reason for the small discrepancy is that small differences in the maximum determined relaxation time can make a deviation in the phenomenological constant. In the measurements of the two DMPC plus $0.1 w t \%$ 1-octanol suspensions maximum relaxation times of $17.7 \mathrm{~s}$ and $17.2 \mathrm{~s}$ were measured. Standard deviations of a few tenth of a second are usually found.

\section{Influence of Neurotransmitters}
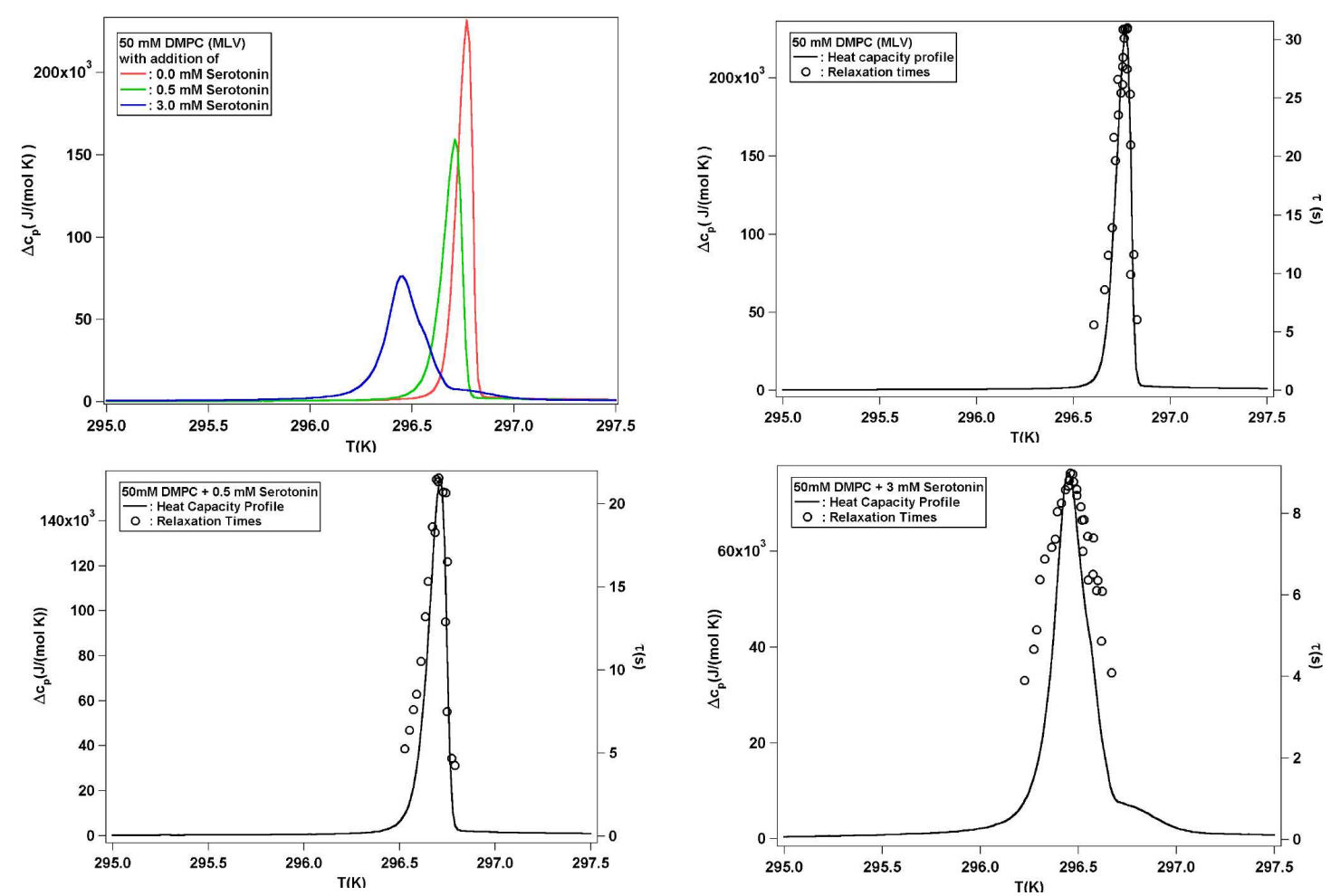

Figure 4.18: Relaxation time measurements on DMPC (MLV) solutions with addition of the neurotransmitter serotonin. Relaxation times (open circles) and heat capacity profiles (solid curves) of DMPC (MLV) membranes were measured after addition of various concentrations of serotonin: (top panel, right) pure DMPC membrane, (bottom panel, left) plus $0.5 \mathrm{mM}$ and (bottom panel, right) with $3 \mathrm{mM}$ serotonin. Higher concentrations of serotonin lead to broader heat capacity profiles, a decrease in the maximum heat capacity value and a shift of the profile to lower temperatures (top panel, left). The maximum relaxation time is also influenced and becomes smaller with increasing serotonin concentration. The temperature region of increased relaxation times is shifted in the same manner like the heat capacity profile. Note the different scaling of the heat capacity and relaxation time axes in the three graphs displaying relaxation times and heat capacity.

Another series of experiments were conducted on a DMPC/Serotonin system (see fig. 4.18). DMPC lipid powder was dissolved in a buffer solution with different concentrations of serotonin. Relaxation times and excess heat capacity display again a linear relationship. 
Not only $\Delta c_{p}$-profiles, but also the relaxation time profiles shift to lower temperatures and broaden. Relaxation times are faster than in DMPC vesicles without serotonin. Relaxation times at the transition midpoint are $\tau_{m}=21.6 \mathrm{~s}\left(1 \mathrm{mM}\right.$ serotonin) and $\tau_{m}=9.0 \mathrm{~s}$ (3 $\mathrm{mM}$ serotonin). Calculated phenomenological constants are $L=6.5 \cdot 10^{8} \frac{\mathrm{JK}}{\mathrm{mol} \mathrm{s}}$ and $L=7.5 \cdot 10^{8} \frac{\mathrm{JK}}{\mathrm{mols}}$, respectively. It has to be mentioned that measurements at two other serotonin concentrations $(1 \mathrm{mM}$ and $2.5 \mathrm{mM})$ showed a proportionality of heat capacity and relaxation time, but phenomenological constants are found to be $11.7 \cdot 10^{8} \frac{\mathrm{JK}}{\mathrm{mols}}$ and $8.7 \cdot 10^{8} \frac{\mathrm{JK}}{\mathrm{mols}}$. In the one case it is almost a factor of two higher than the ones which were found before. In these measurements the deviations seem to arise from a broadening of the phase transition profile. DSC scans of the samples showed that the curves broadened with time. Heat capacity profiles were measured on successive days before the relaxation time measurements. The later scans showed a broader transition regime than earlier ones. This suggests that the actual profiles during the relaxation time measurements were even broader. This again means a smaller maximum heat capacity value and therewith also a smaller phenomenological constant. This presumably also explains the slightly higher value of $L=7.5 \cdot 10^{8} \frac{\mathrm{JK}}{\mathrm{mols}}$ in the measurement with $3 \mathrm{mM}$ serotonin.

\section{Influence of Antibiotics}

Pressure perturbation experiments were also performed with DMPC MLVs and the addition of the antibiotic gramicidin A (measurements were done in collaboration with M.L. Gudmundsson; see (Gudmundsson, 2004)). The used pressure cell was the one of method II, but still the calorimeter response was analyzed. The displayed relaxation times are averages of two measurements at the same temperature. DSC scans on successive days revealed that the profiles differed. The maximum in heat capacity was shifted to sometimes lower and sometimes higher temperatures. The shifts were in the few hundreds of Kelvin regime. Often no broadening was observed, but if there was a broadening it was in a range as also seen in the experiments with addition of neurotransmitters or anesthetics. Based on DSC scans of $10 \mathrm{mM}$ solutions in the calorimeter cell temperatures were corrected. Scans were performed with scan rates of $1 \frac{K}{h}$ or slower. The shown profiles are results with a scan rate of $1 \frac{K}{h}$. From other experiments it is known that the temperature shift between a scan with $5 \frac{K}{h}$ and $1 \frac{K}{h}$ is constant $(\Delta T=-0.03 K)$. We shifted the heat capacity profiles and also the relaxation times with this value of $\Delta T=-0.03 \mathrm{~K}$ so that the transition midpoint temperature equals the expected one for a scan with $5 \frac{K}{h}$. Despite these problems one can conclude that again a proportionality between the heat capacities and relaxation times is found. The maximum relaxation times and the phenomenological constants at the transition midpoint are $\tau_{m}=20.8 \mathrm{~s}$ and $L=7.9 \cdot 10^{8} \frac{\mathrm{JK}}{\mathrm{mol} \mathrm{s}}$ (plus $0.5 \mathrm{~mol} \%$ gramicidin A) and $\tau_{m}=13.1 \mathrm{~s}$ and $L=5.8 \cdot 10^{8} \frac{\mathrm{JK}}{\mathrm{mols}}$ (plus $1.5 \mathrm{~mol} \%$ gramicidin A). The found phenomenological constants deviate from each other. These might be due to errors in the determination of the relaxation times. E.g. in the case of the measurements resulting in a phenomenological constant of $5.8 \cdot 10^{8} \frac{\mathrm{JK}}{\mathrm{mol} s}$ a maximum relaxation time of $12.0 \mathrm{~s}$ would lead to a phenomenological constant of $6.3 \cdot 10^{8} \frac{\mathrm{JK}}{\mathrm{mol} \mathrm{s}}$. Looking at the data one finds that the value which leads to a relaxation time of $13.1 s$ might be an error and the maximum relaxation time is rather around $12.0 \mathrm{~s}$. The higher phenomenological constant as obtained in the measurement on DMPC plus 0.5mol\% gramicidin A might have occurred because of a broadening. 

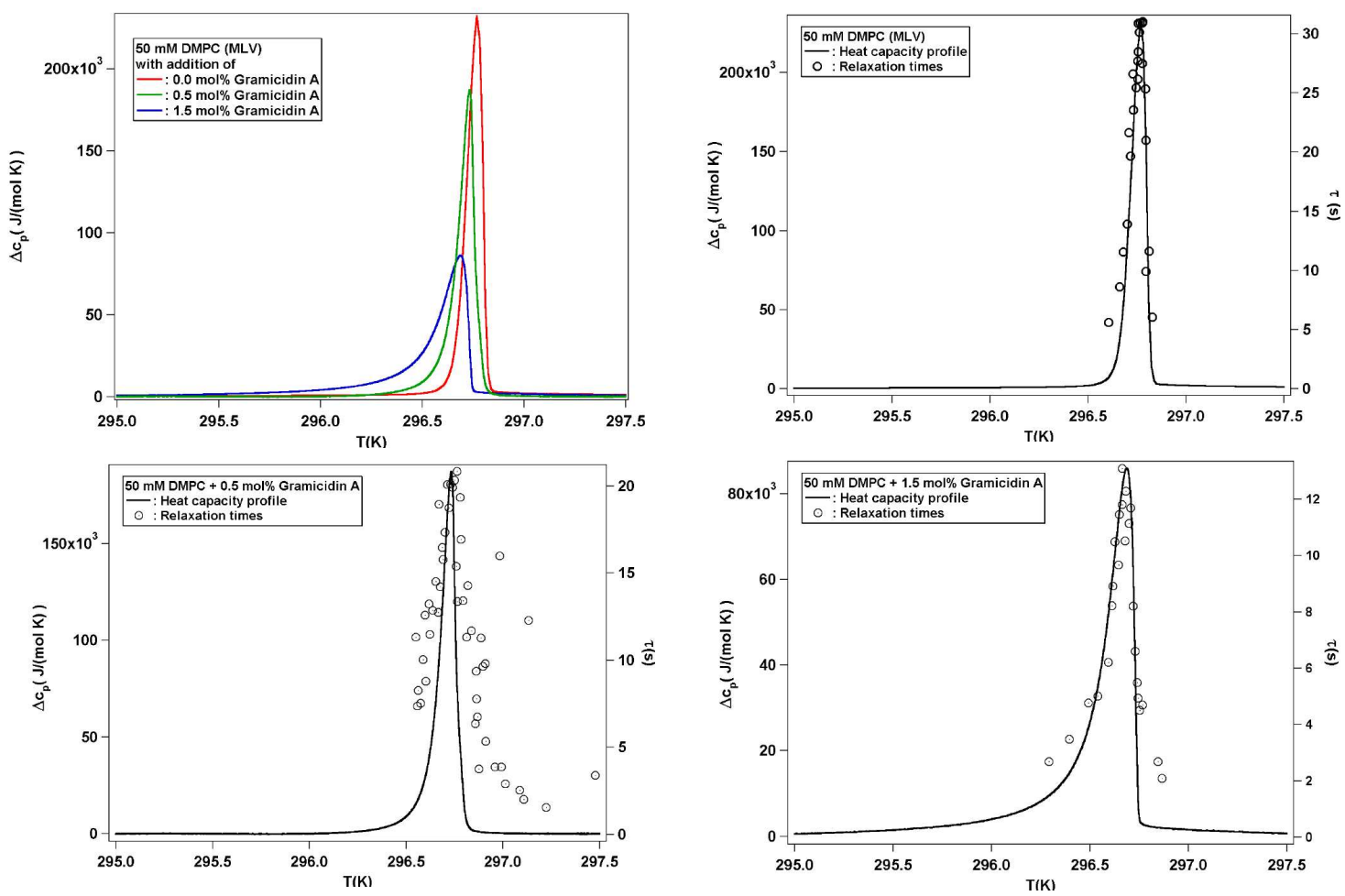

Figure 4.19: Relaxation times of a DMPC plus gramicidin A (MLV) suspension. Heat capacity profiles (solid curves) and relaxation times (open circles) are affected by addition of various concentrations of gramicidin A to DMPC (MLV) membranes: (top panel, right) pure DMPC membrane, (bottom panel, left) with addition of $0.5 \mathrm{~mol} \%$ and (bottom panel, right) $1.5 \mathrm{~mol} \%$ gramicidin A. The maximum relaxation time and the maximum heat capacity become smaller with increasing gramicidin A concentration. The profiles are shifted to lower temperatures and are broadened. For a better visualization the measured heat capacity profiles are plotted in one graph (top panel, left). Note the different sizes of the heat capacity and relaxation time axes in the three graphs displaying relaxation times and the respective heat capacity curve.

\section{Calorimeter Response: Perturbation and Lipid Signal}

In sec. 4.4.1 it has been shown that the total area below the calorimeter response depends on the transition enthalpy and that it is proportional to the released transition enthalpy. The total signal originates from a perturbation of the setup and the release or absorption of heat from the lipids. If there is merely one relaxation process, area of the perturbation signal should stay constant in a small temperature interval. It should not show any temperature dependence. As it can be seen in fig. 4.20 (left panel) the area of the perturbation signal changes as expected if it depends on a contribution originating from the lipids. This signal must be the sum of a perturbation and a lipid contribution of a fast relaxation process. The area of the response belonging to the slow relaxation process (fig. 4.20, right panel) makes about $95 \%$ of the response originating from the lipids. In general, the fast relaxation process accounts for about $5-8 \%$ of the total signal coming from the lipids. Therefore, the contribution of the fast relaxation process is small. 

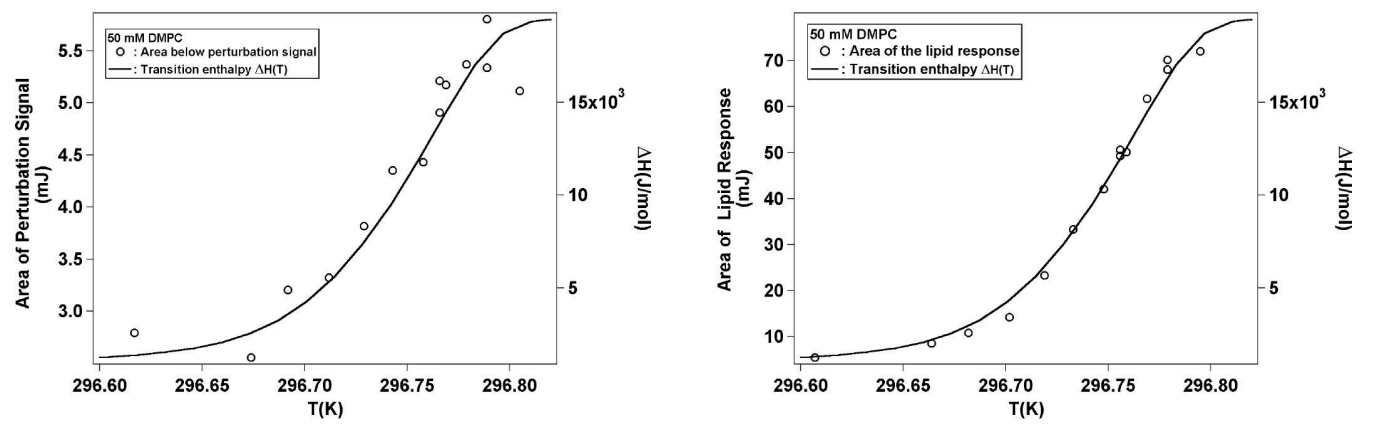

Figure 4.20: Transition enthalpy and area of the perturbation and lipid signal (method I) versus temperature. (left) The temperature dependent transition enthalpy (green, solid curve) and the area of the perturbation signal (open circles) are shown and can be compared. (right) The area of the response originating from the lipid (open circles) is compared to the temperature dependent course of the transition enthalpy (green, solid curve). Both, the response originating from the lipid and the perturbation are proportional to the transition enthalpy. The temperature dependence of the perturbation response reveals a relaxation process below the time resolution limit of the experiment.

\section{Large Unilamellar Vesicles}

Experiments on unilamellar vesicle systems using method II have not revealed any further faster relaxation processes. Temperature versus time curves were fitted with one relaxation process. One explanation of not finding faster processes might lie in the different vesicle systems, another one in the weak contribution of the fast relaxation process. A limitation of the time resolution is a third possible reason.

Concentrations in the experiments with LUVs were chosen to be $40 \mathrm{mM}$. Twice one component lipid suspensions (DMPC and DPPC) and once a lipid/halothane system (DMPC + $20 w t \%$ halothane) were chosen. DPPC membranes melt at higher temperatures than DMPC ones. Halothane shifts the profile to lower temperatures and broadens the profile. In the pressure perturbation experiments the pressure change was +40 bar and at each temperature two pressure jumps were done and relaxation times were averaged. $\Delta c_{p}$-curves were measured at a scan rate of $5 \frac{K}{h}$ and the transition profiles were standardized to $20 \frac{\mathrm{kJ}}{\mathrm{mol}}$ (DMPC membranes) and $30 \frac{\mathrm{kJ}}{\mathrm{mol}}$ (DPPC system). The transition enthalpies were determined through DSC scans directly in the calorimeter cells with $10 \mathrm{mM}$ DMPC and DPPC solutions.

A proportionality between heat capacity and relaxation time was found in all measurements (fig. 4.21). The maximum relaxation times were measured to be $\tau_{m}=1.5 \mathrm{~s}$ (40 $\mathrm{mM}$ DMPC), $\tau_{m}=1.0 \mathrm{~s}\left(40 \mathrm{mM} \mathrm{DMPC}+20 w t \%\right.$ halothane) and $\tau_{m}=2.0 \mathrm{~s}(40 \mathrm{mM} \mathrm{DPPC})$. These values and the respective heat capacity and melting temperature values allow to estimate the three phenomenological constants $L=15.7 \cdot 10^{8} \frac{\mathrm{JK}}{\mathrm{mol} \mathrm{s}}\left(40 \mathrm{mM}\right.$ DMPC), $L=20.0 \cdot 10^{8} \frac{\mathrm{JK}}{\mathrm{mol} \mathrm{s}}(40 \mathrm{mM}$ DMPC plus $20 w t \%$ halothane) and $L=18.4 \cdot 10^{8} \frac{\mathrm{JK}}{\mathrm{mol} \mathrm{s}}(40 \mathrm{mM} \mathrm{DPPC})$. These values differ, but it seems that one can estimate an averaged value of $(18.0 \pm 2.16) \cdot 10^{8} \frac{\mathrm{JK}}{\mathrm{mol} \mathrm{s}}$.

At the upper and lower temperature limits of the transition bigger deviations from the proportionality can be found. This is possibly due to too weak lipid signals at especially the upper temperature limit. The limited time resolution of around $500 \mathrm{~ms}$ is another explanation. 


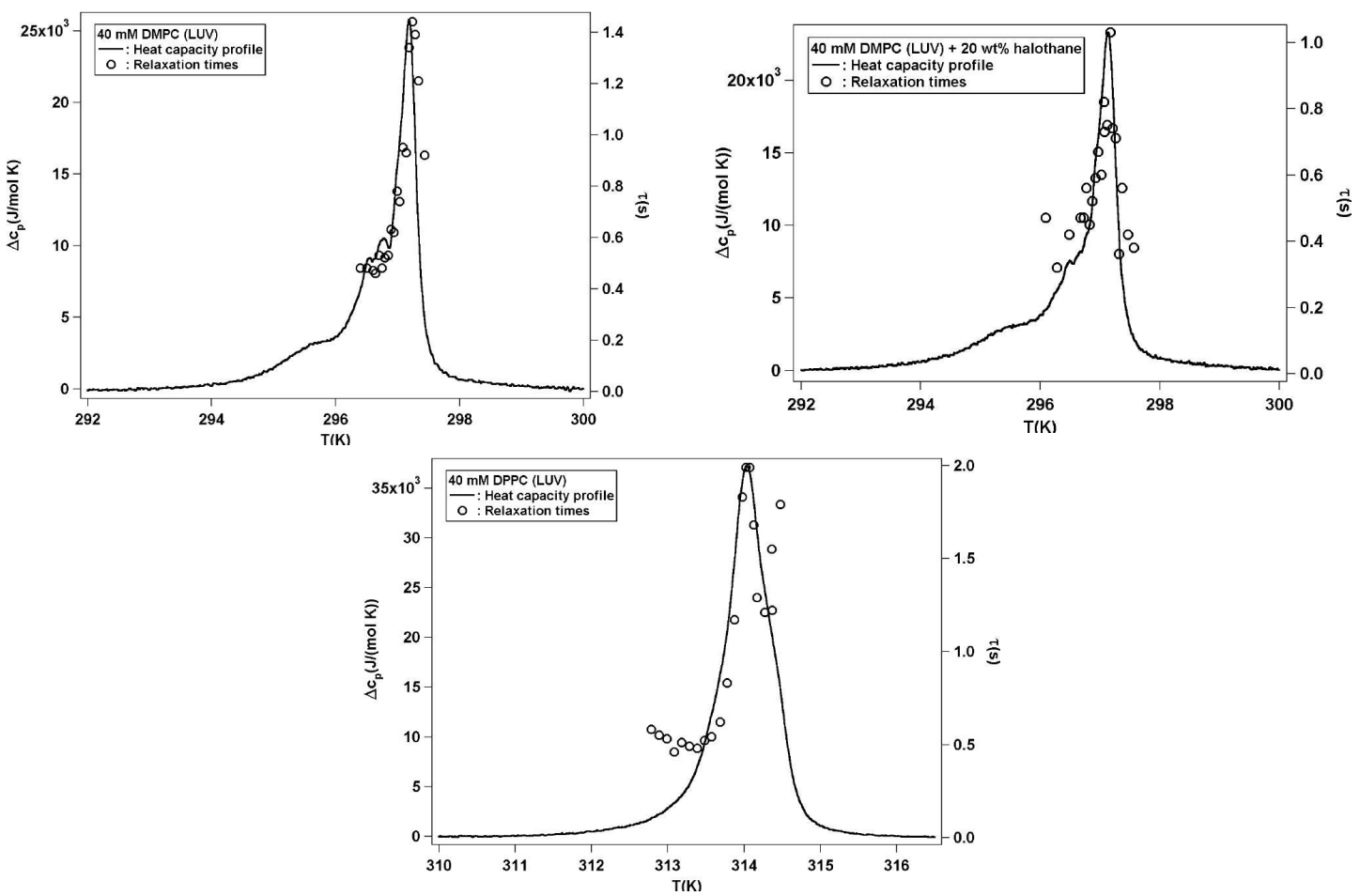

Figure 4.21: Relaxation times of large unilamellar vesicle systems. Heat capacity profiles (solid curves) of DMPC (top panel, left), DMPC plus $20 w t \%$ halothane and DPPC (bottom panel) LUVs are displayed. Relaxation times (open circles) of these membrane systems were collected after analysis of the temperature development of the sample solution after a ppj. Halothane broadens the heat capacity profile of DMPC LUVs and shifts it to lower temperatures in comparison to the measurement without halothane. This affects the maximum heat capacity value and maximum relaxation time. DMPC LUVs melt at lower temperatures than DPPC LUVs. The temperature regime of slowed down relaxation processes lies at lower temperatures in the case of DMPC and at higher temperatures in the case of DPPC. The maximum heat capacity of the profile measured with the DPPC LUV suspension is higher than the one determined with DMPC LUVs. The maximum relaxation time of DPPC LUVs is longer than the one of DMPC LUVs. In all three measurements it is found again that relaxation times are proportional to heat capacity.

\section{Linearity between Relaxation Times and Heat Capacity}

In fig. 4.22 relaxation times from all measurements are plotted against respective heat capacity values. The left panel shows results with multilamellar vesicles, with and without addition of non-lipid molecules (red circles), with DMPC LUVs, with and without halothane (blue squares) and with DPPC LUVs (green crosses). The obtained results with LUVs are also shown in the right panel of fig. 4.22. Solid and dashed lines are respective linear fits. It is evident that none of the fits intersects with the origin as expected from the theoretical findings $\left(\tau=\frac{T^{2}}{L} \Delta c_{p}\right.$; see also sec. 3.2). It seems, however, that this is because of experimental and evaluation limitations rather than a physical characteristic of the system. The response of the calorimeter does not allow a determination of relaxation processes faster than $3-4 s$. Faster relaxation processes are detected monitoring directly the temperature of the sample 

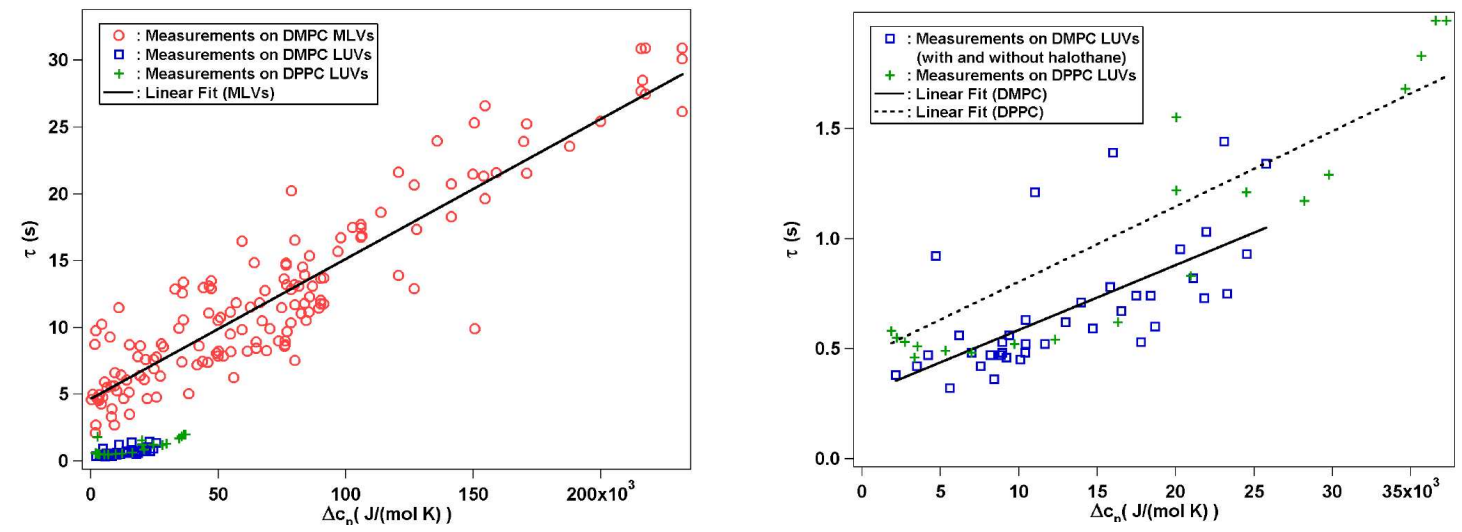

Figure 4.22: Relaxation time vs. heat capacity plots. (left) Relaxation times from all measurements are plotted against respective heat capacities. Measurements with DMPC MLVs with or without addition of other molecules are marked with red, open circles. A linear fit (black, solid curve) of these measurements is drawn too. It does not intersect the origin. Results from measurements with DMPC LUVs with and without addition of halothane (blue, open squares) and DPPC LUVs (green crosses) are included too. (right) The obtained results from pressure perturbation experiments with LUVs are drawn in a separate graph. Measurements with DMPC LUVs are again marked by blue squares, where green crosses mark results from suspension of DPPC LUVs. The black, solid and the black, dashed line are respective linear fits. In all graphs the scaling of the heat capacity and relaxation time axes differ.

solution. The resolution limit is about $300-400 \mathrm{~ms}$. It could be argued that with this method one should have obtained shorter relaxation times at the upper and lower temperature limit of the transition. This was, however, not the case (see fig. 4.15). This again can be attributed to experimental reasons. Temperature curves were collected after ppjs. This means that at the upper temperature limit lipid signals are weak and the determination of relaxation times is difficult. At the lower temperature limit the signal originating from the lipid is strong since one jumps through a major part of the transition. This induces relatively big temperature changes. For MLVs these changes might mean that one obtains a temperature evolution which is dominated by slower relaxation processes as they would have been expected from the temperature given by the calorimeter.

We propose that relaxation times and heat capacity are proportional to each other. The reason why linear fits do not intersect the origin arise probably because of experimental limitations.

\section{Summary}

In table 4.1 all results of the maximum relaxation times and the corresponding phenomenological constants are given. Concerning the measurements on MLVs in especially two cases phenomenological constants deviate strongly from the other measured ones. Still it seems that the phenomenological constant does not depend on the lipid membrane system. Neglecting the two exceptions one finds an average of $(6.7 \pm 0.7) \cdot 10^{8} \frac{\mathrm{JK}}{\mathrm{mols}}$. The phenomenological constants of the LUV suspensions differ from the ones of the MLVs. They suggest, however, that they are constant for the LUV suspensions with a mean value of $(18.0 \pm 2.2) \cdot 10^{8} \frac{\mathrm{JK}}{\mathrm{mol} \mathrm{s}}$. 


\begin{tabular}{|l|c|c|}
\hline Lipid Membrane System & $\tau_{m} / s$ & $L /\left(10^{8} \frac{\mathrm{JK}}{\mathrm{mol} s}\right)$ \\
\hline \hline Method I & 31.0 & 6.6 \\
\hline \hline DMPC (MLV) & 17.7 & 6.3 \\
\hline DMPC + 0.1 $w t \%$ 1-octanol (MLV) & 14.8 & 6.3 \\
\hline DMPC + 0.5 $w t \%$ 1-octanol (MLV) & 21.6 & 6.5 \\
\hline DMPC + 0.5 mM serotonin (MLV) & 9.0 & 7.5 \\
\hline DMPC + 3.0 mM serotonin (MLV) & 20.8 & 7.9 \\
\hline DMPC + 0.5 mol\% gramicidin A (MLV) & 13.1 & 5.8 \\
\hline DMPC + 1.5 mol\% gramicidin A (MLV) & \multicolumn{3}{|l|}{} \\
\hline \hline Method II & 25.2 & 6.8 \\
\hline \hline DMPC (MLV) & 1.5 & 15.7 \\
\hline DMPC (LUV) & 1.0 & 20.0 \\
\hline DMPC + 20 $w t \%$ halothane (LUV) & 2.0 & 18.4 \\
\hline DPPC (LUV) &
\end{tabular}

Table 4.1: Relaxation times and phenomenological constants at the transition midpoint of various studied systems.

It can be summarized that the prediction from Grabitz et al. (2002) (see eq. 3.31) that relaxation times are proportional to heat capacity is true for one component lipid systems with and without addition of small molecules and peptides. Addition of anesthetics, neurotransmitters or antibiotics leads to a decrease of the maximum relaxation time and shifts the region of slow relaxation processes to lower temperatures. The theory by Grabitz and collaborators relates relaxation processes to fluctuations in enthalpy. It is, however, not automatically clear whether relaxation times from pressure perturbation experiments are equivalent to the time scales of enthalpy fluctuations.

In the next section simulated temperature jump experiments are used for the study of the influence of different temperature jump sizes on the relaxation behavior. These results can be compared to the autocorrelations of simulated enthalpy traces. Later, this allows one to discuss whether relaxation times from our pressure perturbation experiments are related to fluctuations in enthalpy and whether they give the same time scale.

\subsubsection{Temperature Jump Experiments: Simulation}

As it is shown in the previous section relaxation times and the respective heat capacities display a linear relation. This is true for one component phospholipid systems independent of the vesicle type, as well for one component phospholipid systems with the addition of small molecules or peptides. These results are explained by the introduced non-equilibrium thermodynamics theory of Grabitz et al. (2002) (see also sec. 3.2). This theory relates fluctuations in enthalpy to relaxation processes. The experimentally determined relaxation times are measured after pressure changes which result in rather big enthalpy changes. Therefore, the question arises whether time scales of the enthalpy fluctuations and relaxation times obtained after pressure perturbations are equal. 


\section{Time Dependence of Lipid Chain State Distribution}

MC simulations using the introduced numerical model (see sec. 3.1.2) allow one to study the differences of relaxation times as obtained from enthalpy fluctuations in the equilibrium and from enthalpy courses after a jump in temperature. Temperature changes are equivalent to pressure changes.
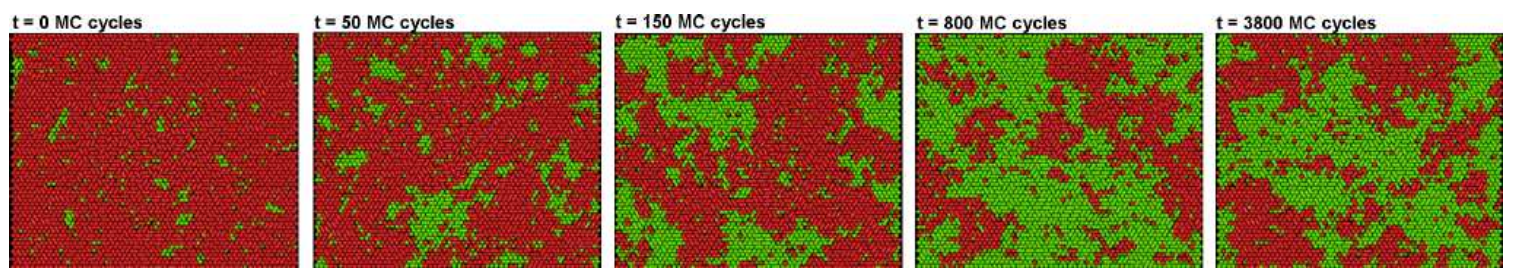

Figure 4.23: Snapshots of the distribution of lipid chain state after a temperature jump. A one component lipid system with $\Delta H=13168 \mathrm{~J} /($ mol chain $), \Delta S=44.31 \mathrm{~J} /($ mol chain $K)$ and an interaction parameter of $\omega=1269.94 \mathrm{~J} /(\mathrm{mol}$ chain $)$ was equilibrated at a temperature of $290.0 \mathrm{~K}$. At time $t=0 \mathrm{MC}$ cycles the temperature was increased to $297.1 \mathrm{~K}$, so one jumps into the transition regime. Snapshots of the lipid chain state distribution are plotted as a function of time. Ordered chains are painted red, whereas disordered are green.

MC simulations also allow one to follow the state distribution of the lipid chains with time. A one component lipid system with $\Delta H=13168 \mathrm{~J} /($ mol chain $), \Delta S=44.31 \mathrm{~J} /($ mol chain $K)$, $\omega=1269.94 \mathrm{~J} /(\mathrm{mol}$ chain $)$ and $T_{m}=297.1 \mathrm{~K}$ was chosen. The matrix size was set to 60 chains $\times 60$ chains. After equilibration of the system the temperature was changed from $290.0 \mathrm{~K}$ to $297.1 \mathrm{~K}$. At time $t=0 \mathrm{MC}$ cycles the lipid chains are mainly in the ordered state. A jump into the transition regime leads to melting of lipid chains and to the formation of domains of ordered and disordered lipid chains (see fig. 4.23). This is reflected by a characteristic time. Analyzing snapshots, however, does not allow one to determine a relaxation time. One possibility is monitoring the time course of the change in enthalpy or the number of ordered or disordered chains after a temperature jump.

\section{Temperature Jump Simulations: One Component Lipid Membrane}

The same values for the enthalpy and entropy change as above were used to perform temperature jump simulations with two different interaction parameters $(\omega=1186.24 \mathrm{~J} /($ mol chain $)$ and $\omega=1269.94 \mathrm{~J} /($ mol chain $))$. The matrix size was set to 60 chains $\times 60$ chains. At first $10000 \mathrm{MC}$ cycles were conducted to equilibrate the system. Then the simulation of the equilibrated lipid system was performed. After another $200 \mathrm{MC}$ cycles the temperature was changed to the transition midpoint temperature $\left(T_{m}=297.1 \mathrm{~K}\right)$. The initial temperature was varied. For each starting temperature five simulations were carried out. Relaxation times from these simulations were averaged.

One question to answer is whether the initial temperature has an influence on the relaxation time. In fig. 4.24 two enthalpy curves after a temperature change are displayed. In this case the interaction parameter equaled $\omega=1269.94 \mathrm{~J} /($ mol chain $)$. The two starting temperatures are above and below the transition midpoint temperature. In the one case one jumps from 


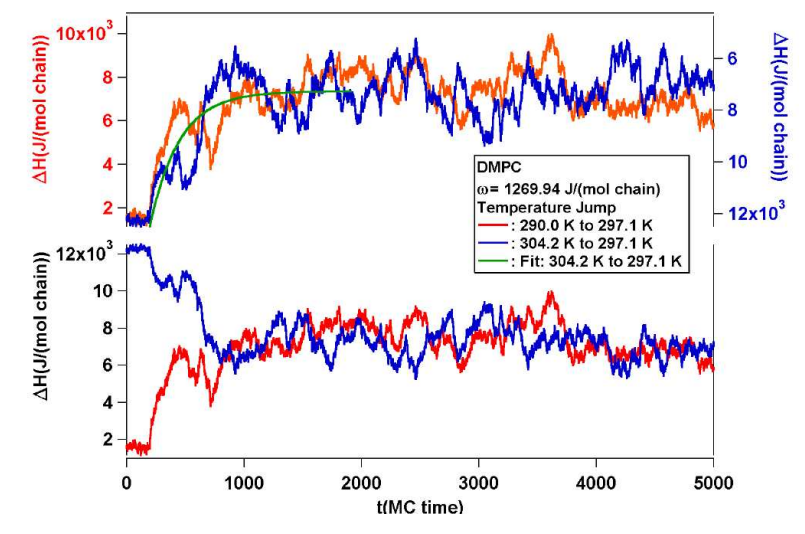

Figure 4.24: Simulated temperature jump experiments. A one component lipid system with $\Delta H=$ $13168 \mathrm{~J} /($ mol chain $), \Delta S=44.31 \mathrm{~J} /($ mol chain $K)$ and an interaction parameter of $\omega=1269.94 \mathrm{~J} /($ mol chain $)$ was used for the simulation of temperature jump experiments. Shown are results of jumps into the transition midpoint from temperatures below (red) and above (blue) the transition regime. In the upper panel the enthalpy axis of the jump from $304.2 \mathrm{~K}$ to $297.1 \mathrm{~K}$ is reversed (right, upper axis), so one can easily compare the time scales of the two curves. The green curve is the respective single exponential fit belonging to the enthalpy course which was obtained after the jump from $304.2 \mathrm{~K}$ to $297.1 \mathrm{~K}$.

the "so" $(290.0 K \rightarrow 297.1 K)$ and in the other case from the "ld" $(304.2 K \rightarrow 297.1 K)$ phase into the transition regime. This means that changing temperature leads to an increase $(290.0 \mathrm{~K} \rightarrow 297.1 \mathrm{~K}$; bottom panel, red curve) or decrease $(304.2 \mathrm{~K} \rightarrow 297.1 \mathrm{~K}$; bottom panel, blue curve) in enthalpy. After the system is relaxed into its equilibrium both enthalpy curves fluctuate as expected and required around the equilibrium value. The time scale of the relaxation seems to be the same. For a better recognition the enthalpy axis of the jump from the temperature above the transition midpoint is reversed (fig. 4.24 top panel).

The time development of the enthalpy curves can be described by a one component exponential fit. The respective fit of the jump from $304.2 \mathrm{~K}$ to $297.1 \mathrm{~K}$ is displayed (green curve). Averaging relaxation times from five simulations at the same initial temperature we found times of $272 \pm 123 \mathrm{MCcycles}(290.0 \mathrm{~K} \rightarrow 297.1 \mathrm{~K})$ and $242 \pm 62 \mathrm{MCcycles}(304.2 \mathrm{~K} \rightarrow 297.1 \mathrm{~K})$. The determined relaxation times are of the same order.

The deviations are possibly due to statistical uncertainties. This seems that averaging with results from just five curves is not sufficient and averaging of more curves would be necessary. Because of the strong fluctuations in enthalpy at the transition midpoint one sometimes finds ambiguous fitting results. This might explain that results from jumps with other starting temperatures result in higher relaxation times like $323 \pm 241 \mathrm{MC}$ cycles or $345 \pm 162 \mathrm{MC}$ cycles. Averaging all fitted relaxation times one obtains a relaxation time of $289 \pm 140$ MCcycles. This is in the time range of the relaxation time which is calculated from the autocorrelated enthalpy traces as it is presented in sec. 3.2. In the evaluation of the autocorrelation profiles of enthalpy traces at a fixed temperature one finds that at the transition midpoint $(297.1 K)$ the relaxation is as high as $307 \pm 2 M C$ cycles. Relaxation times received from temperature jump simulations and from autocorrelations of enthalpy traces presumably equal. This statement probably is valid, even though the average relaxation time from the temperature jump simulations is shorter, but shows a high standard deviation. The same is true for the simulations conducted 
with a lower interaction parameter of $\omega=1186.24 \mathrm{~J} /($ mol chain $)$. The relaxation time from an autocorrelation curve of an enthalpy trace at $297.1 \mathrm{~K}$ is $71 \pm 2 \mathrm{MC}$ cycles, whereas the averaged relaxation time from the temperature jump is $61 \pm 20 \mathrm{MC}$ cycles. The obtained relaxation times do not depend on the size of the lipid system (data not shown). This is expected because the heat capacity value at one temperature is independent of the matrix $\operatorname{size}^{5}$. If heat capacities are the same relaxation times should also stay constant.
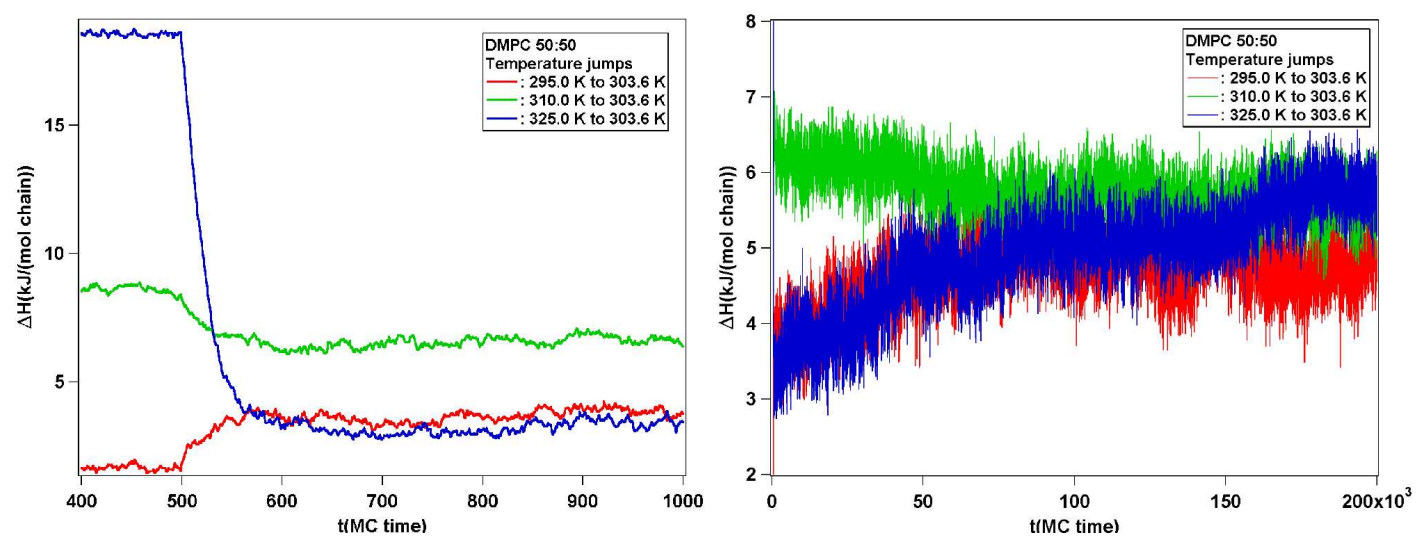

Figure 4.25: Temperature jump simulation of a two component lipid mixture. An equimolar DMPC:DSPC system was chosen to perform temperature jump experiments from different temperatures to a temperature of $303.6 \mathrm{~K}$. The temperature was changed after the first $500 \mathrm{MC}$ cyles in the equilibrated system. The whole trace was followed over $0.5 \cdot 10^{6} \mathrm{MC}$ cyles. (left) After the temperature change the first $500 \mathrm{MC}$ cyles are displayed. (right) The trace is plotted over a longer time. One sees that in all three cases the enthalpy fluctuates around an equilibrium value at later times. There are long and short relaxation processes.

Due to statistical uncertainties one cannot make an unambiguous statement, but it seems that relaxation times obtained from enthalpy fluctuations in the equilibrium and from temperature jumps equal each other. Relaxation times do not depend on starting temperature. This means that our pressure jump experiments result in time scales which equal the ones of enthalpy fluctuations in equilibrium.

\section{Temperature Jump Simulations: Binary Mixtures}

So far we have not been able to perform successful systematic experiments on two component lipid mixtures. Again using MC simulations we can mimic a pressure jump experiment on a lipid mixture.

Temperature jump simulations were performed on a DMPC:DSPC 50 : 50 mixture. The system size was chosen to be 80 chains $\times 80$ chains. Three initial temperatures were chosen $(295.0 \mathrm{~K}, 310.0 \mathrm{~K}$ and $325.0 \mathrm{~K})$. After equilibration of the system at the starting temperature, temperatures were not changed for the first $500 M C$ cyles. After that the system's temperature was instantaneously changed to $303.6 \mathrm{~K}$ and the enthalpy values were monitored up to a time of $0.5 \cdot 10^{6} M C$ cylces. Results are plotted in fig. 4.25. In the left panel the enthalpy developments for the first $500 \mathrm{MC}$ cycles after the temperature change can be followed. In the right panel the enthalpy course is shown for $200000 \mathrm{MC}$ cycles. It is obvious that the enthalpy

\footnotetext{
${ }^{5}$ This is at least true for matrix sizes bigger than a critical one (finite size scaling; see sec. 4.1).
} 
curves cannot be fitted by one relaxation term. The enthalpy development during the first $500 M C$ cycles after a temperature jump, however, can be described by a single exponential fit. All three curves can be fitted with the same time constant of about $40 \mathrm{MC}$ cycles just considering the first $500 \mathrm{MC}$ cycles after the change in temperature. We find, that after the first $500 \mathrm{MC}$ cycles after the pressure jump in the two cases when jumping from outside of the transition regime into it ( $295.0 \mathrm{~K}$ to $303.6 \mathrm{~K}$ and $325 \mathrm{~K}$ to $303.6 \mathrm{~K}$ ) enthalpy fluctuations seem to be around the same average. When jumping from a point in the transition regime $(310.0 \mathrm{~K})$ to the point at $303.6 \mathrm{~K}$ fluctuations are around another averaged enthalpy. At long times all enthalpy curves fluctuate around the same averaged value of about $1212 \frac{\mathrm{J}}{\mathrm{mol} \text { chain }}$. This one is as anticipated equal to the average value one finds in equilibrium simulations. This means that the relaxation behavior is more complex and it cannot be explained by one relaxation process. At least one fast and one slow process exist. Evaluating the enthalpy curve after a temperature jump does not give any unambiguous information. The statistics of the system would require the evaluation of many simulations. Therefore, it is more favorable to study the timescales of enthalpy fluctuations in the equilibrated system through an autocorrelation of enthalpy traces.

\subsubsection{Relaxation Processes of DMPC:DSPC Mixtures: Simulation}

With the help of temperature jump simulations on binary lipid mixtures of DMPC:DSPC it is found that the relaxation behavior of binary mixtures can be more complex than for one component lipid systems (see previous section). The time scale of enthalpy fluctuations of one component systems is related to relaxation processes (see previous section and sec. 3.2). Similarly to one component systems autocorrelation profiles of fluctuations in enthalpy of two component lipid systems in equilibrium can be used to study the behavior of relaxation processes.

\section{Dependence of Relaxation Processes on Temperature}

In the left panel of fig. 4.26 the black curves are autocorrelation profiles of enthalpy traces at different temperatures of a DMPC:DSPC 50 : 50 mixtures (system size: 60chains $\times 60$ chains). In each simulation $10^{6}$ enthalpy data points were recorded and autocorrelated. The shapes of the autocorrelation profiles depend on temperature. The corresponding simulated heat capacity profile (right panel of fig. 4.26) allows to find out that heat capacity values and the phase state at the four different temperatures differ. At $290.5 \mathrm{~K}$ and $322.5 \mathrm{~K}$ the lipid membrane is in the "so" or "ld" phase, respectively. The small values in heat capacity mean that enthalpy fluctuations are small. From the decay of the autocorrelation curves can be followed that fluctuations are small and happen on a fast time scale. This changes at temperatures when domains coexist $(303.6 \mathrm{~K}$ and $309.7 \mathrm{~K})$. At a temperature of $303.6 \mathrm{~K}$ the heat capacity is the highest comparing the values at the chosen temperatures. Therefore, enthalpy fluctuations are also the largest. The decay of the autocorrelation profile is the slowest of the shown profiles. Short and long relaxation processes must be present. This is also true at a temperature of $309.7 \mathrm{~K}$. At this temperature, however, enthalpy fluctuations are not as strong as at $303.6 \mathrm{~K}$ and the autocorrelation profile decays faster. These findings agree with the ones from the temperature jump simulations of a final temperature of $303.6 \mathrm{~K}$ (see fig. 4.25). In our experiments we would probably monitor the fast process, since this one is accompanied with a relatively strong change in enthalpy. The slower process results in rather 

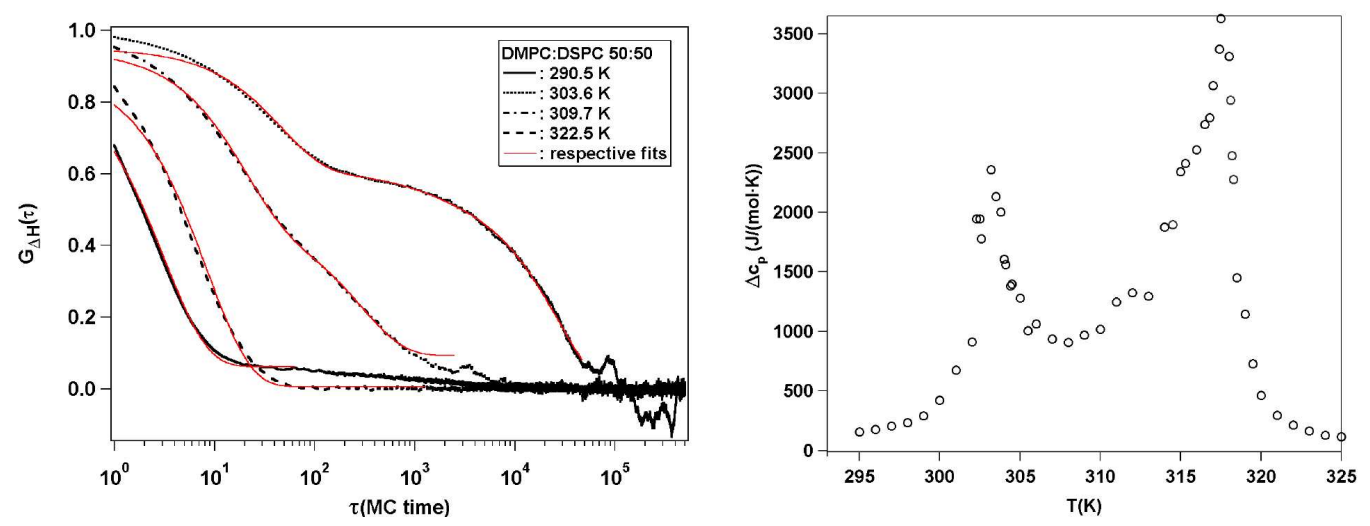

Figure 4.26: (left) Autocorrelation curves of enthalpy fluctuations. Enthalpy traces of a DMPC:DSPC 50 : 50 mixture at different temperatures were autocorrelated. The profiles show that relaxation processes can be more complex than previously described. Phenomenologically, these curves can be fitted with one, two or three relaxation times. Red curves are respective fits. Relaxation times can take up to about $2.7 \cdot 10^{4} \mathrm{MCcycles}$ (Hac et al., 2005). (right) The simulated heat capacity profile of an equimolar DMPC:DSPC membrane is displayed. Comparing the heat capacity values at the temperatures of the corresponding autocorrelation profiles one finds that they differ. At the temperature of the highest heat capacity value $(303.6 \mathrm{~K})$ the autocorrelation curve decays the slowest.

small enthalpy changes. The release or absorption of heat would therefore be stronger in the first case. The release or absorption of heat is what we measure in our pressure perturbation experiments.

Phenomenologically, we tried to fit the autocorrelation profiles with one, two or three component exponential fits. The red curves in fig. 4.26 are the respective fits of the simulated profiles. Fluorescence intensity autocorrelation profiles depend on the diffusion time scales and the time scales of chain state fluctuations (see fig. 4.6). Fits using two diffusion components or assuming anomalous diffusion gain good fit results, but they do not allow a deeper understanding of the underlying physics. This proposes that one should take great care fitting the enthalpy autocorrelation profiles with phenomenological functions.

The autocorrelation curves at $290.5 \mathrm{~K}$ and $322.5 \mathrm{~K}$ are described with a single exponential term. At $290.5 K$ the profile decays slowly at longer times. This decay is not considered in the fit. The profiles at $309.7 \mathrm{~K}$ and $303.6 \mathrm{~K}$ are described by two or respective three exponential terms. At a temperature of $309.7 \mathrm{~K}$ the fit might also suggest that at even longer times a third term would give a better description. In the first hand, it is surprising that using just two MC steps (changes in chain state and diffusion) three relaxation processes shall be present. This might propose that there are physical properties which lead to autocorrelation profiles which seem to reflect more than two relaxation processes, which are actually not present.

\section{Diffusion and Fluctuations in Chain State}

In an earlier section (see sec. 4.2) it is described that diffusion processes are sensitive to variations in the ratio between chain state changes and diffusion steps. This was also tested 

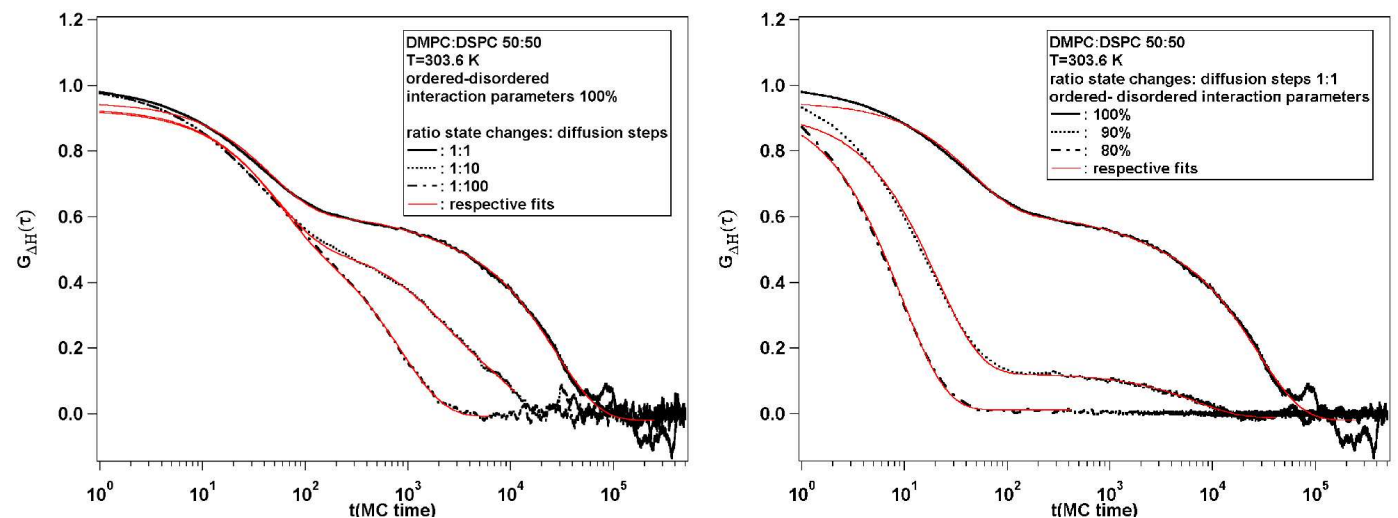

Figure 4.27: Enthalpy correlation dependence on the rate of state changes and interaction parameters. Enthalpy traces of an equimolar DMPC:DSPC system at $303.6 \mathrm{~K}$ were simulated. (left) The ratio between state changes and diffusion steps was changed. (right) The ratio was kept constant, but the ordered-disordered interaction parameters were varied. Both effects influence the shape of the autocorrelation profile. Red curves in both graphs are respective phenomenological fits.

for autocorrelation profiles of enthalpy fluctuations. Enthalpy values were recorded each time the algorithm for the state changes was finished. Traces of a length of $1 \cdot 10^{6} \mathrm{MC}$ cycles were monitored and autocorrelated. The number of diffusion steps between state changes was varied $(1,10$ or 100). Simulations were performed on different systems (DMPC:DSPC $70: 30,50: 50$ and $30: 70)$ at various temperatures. From the comparison of the experimental with the simulated fluorescence intensity autocorrelation profiles we cannot draw a definite conclusion about the ratio of chain state changes to diffusion steps. It seems that the ratio is rather $1: 100$ or $1: 1000$ instead of $1: 1$.

The black curves in the left panel of fig. 4.27 were obtained from simulations of an equimolar DMPC:DSPC mixture at $303.6 \mathrm{~K}$. They show that the shape of the enthalpy autocorrelation profile is dependent on the rate of diffusion steps and the rate of chain state changes. Relaxation processes are influenced by the rates of the two steps.

Phenomenological fits show that found fast time scales are independent of the diffusion rate, where relaxation on longer time scales is influenced by the ratio of the two MC steps.

As mentioned before temperature jump simulations to a final temperature of $303.6 \mathrm{~K}$ show that there is a fast process resulting in relatively big enthalpy changes and a slow process accompanied with small changes. After the fast relaxation enthalpies start to fluctuate around an average which depends on the initial temperature. However, at long times all traces show fluctuations around the same average, which is independent of the temperature before the jump (see fig. 4.25). In this context it seems that the fast relaxation process is due to changes in chain state. The slow relaxation process is dominated by diffusion.

The heat capacity profile of fig. 4.26 was calculated from simulations with the parameters as they are needed for the description of the experimentally measured phase behavior of the DMPC:DSPC system. Reducing the ordered-disordered interaction parameters to $90 \%$ or $80 \%$ of the actual values (see table 3.1) lead to a broadening of the heat capacity profile (see fig. 4.28). At a temperature of $303.6 \mathrm{~K}$ this means that the respective heat capacities get smaller with decreasing interaction parameters. From the fluctuation-dissipation theorem it automatically follows that macroscopic fluctuations become smaller too. From the right 


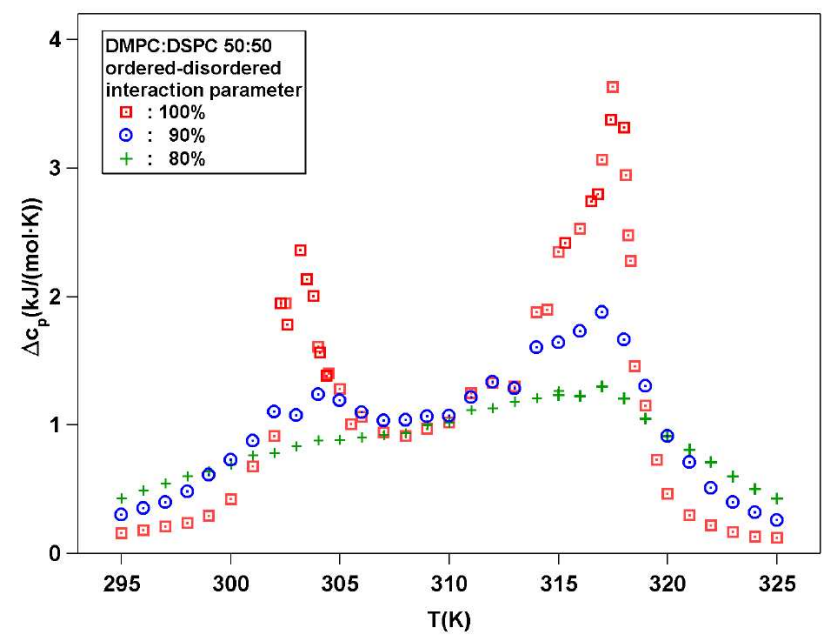

Figure 4.28: Heat capacity profiles of an equimolar DMPC:DSPC mixture with different ordered-disordered interaction parameters. Lowering the interaction parameters between ordered and disordered chains to $90 \%$ or $80 \%$ of their original values (see table 3.1 ) affects the melting profile. The profile broadens with lower interaction parameters and therefore the maximum values decrease. Higher interaction parameters mean that contacts between ordered and disordered chains are less favorable than with lower parameters.

panel of fig. 4.27 it follows that relaxation processes become faster. It is also found that the time scales of the two relaxation processes get closer to each other, which can be seen from the curve from the simulation with interaction parameters reduced to $80 \%$. The profile can be described by one relaxation time. This and the finding that curves can be fitted assuming one, two or three exponential terms suggest that the relaxation processes cannot be separated.

\section{Fluctuations in the Fraction of Disordered Lipid Chains}

Macroscopic fluctuations in enthalpy are related to the fraction of disordered lipid chains. In the MC simulation one can also directly count the total number of disordered chains or differentiate between the two lipid species. In fig. 4.29 the fractions of disordered DMPC and DSPC lipid chains of a DMPC:DSPC $50: 50$ mixture at $303.6 \mathrm{~K}$ are plotted. Interaction parameters are the original ones of table 3.1 and the ratio of state changes to diffusion steps was set to $1: 1$. The strength of fluctuations in the fraction of disordered lipid chains of DMPC and DSPC differ. The fraction of disordered chains of the DMPC lipids fluctuates stronger than the one of the DSPC chains. DMPC membranes melt at lower temperatures than DSPC ones. Therefore, in the vicinity of the first peak of the heat capacity profile mainly DMPC chains melt. The first maximum of the heat capacity profile of a DMPC:DSPC mixture is at $303.2 \mathrm{~K}$. Therefore, at a temperature of $303.6 \mathrm{~K}$ DMPC chain state fluctuations are stronger. It can be shown that this changes at the second heat capacity peak (Seeger, 2002). Analyzing the autocorrelation profiles of the fluid fractions one finds that the relaxation properties of both lipid species are the same and they agree with the ones obtained from the enthalpy fluctuations. 


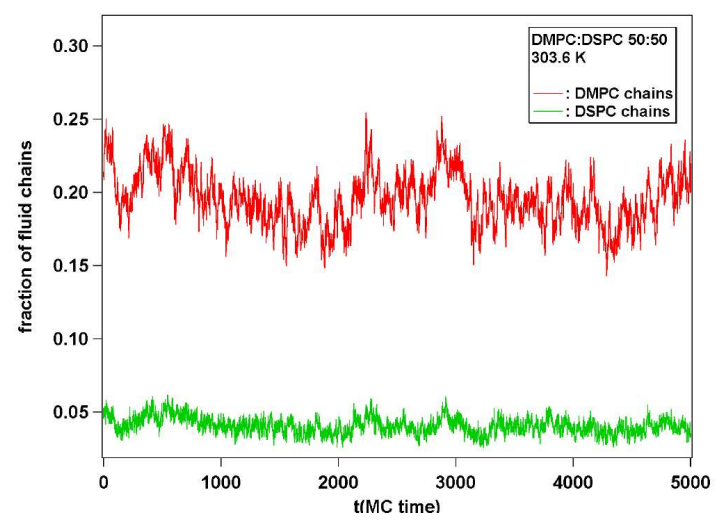

Figure 4.29: Fraction of disordered DMPC and DSPC chains. From the simulations one can extract information about the fraction of disordered chains. Here, the fractions of DMPC and DSPC disordered lipid chains were obtained from a simulation of a DMPC:DSPC $50: 50$ mixture at $303.6 \mathrm{~K}$. The ratio between state changes and diffusion steps was set to $1: 1$. The ordered-disordered interaction parameters equaled the original set of parameters. The strength of the fluctuations differs. The fraction of disordered DMPC chains fluctuates stronger.

\section{Summary}

It is found that the relaxation behavior of lipid mixtures is more complex than the one of one component lipid systems. A fast relaxation process is due to changes in chain state, where a slow one is diffusion controlled. These processes cannot be seen independently from each other. Phenomenological fitting of the autocorrelation profiles is successful, but does not allow a correct description of the underlying physical principles. The two relaxation processes cannot be separated. High values in heat capacity indicate strong and slow fluctuations in enthalpy.

\subsection{Domain Formation and Fluctuations in Lipid Membranes}

One advantage of applying MC simulations to the study of the phase behavior of lipid systems is that one does not only obtain information about thermodynamical averages, but one can also deduce information about the distribution of lipid chain states and fluctuations in chain state (see fig. 4.30). The lipid chain state distribution gives the matrix configuration during one particular MC cycle. Local fluctuations in chain state are an average property. They are calculated by assigning a state parameter $S(i, j, t)$ to chain, $(i, j)$, at time, $t$, and calculating the mean square deviation. Values of $S(i, j, t)=0$ and $S(i, j, t)=1$ are given to an ordered and disordered chain, respectively. Fluctuations can assume values between 0.25 and 0 . In the snapshots of local fluctuations large fluctuations are indicated through a yellow color, where low ones are black. Simulations were performed with an equimolar DMPC:DSPC mixture of size 80 chains $\times 80$ chains at various temperatures. At each of the temperatures simulations were run with three different sets of ordered-disordered interaction parameters. They were set to 100\% (fig. 4.30 top), $90 \%$ (fig. 4.30 center) and $80 \%$ (fig. 4.30 
ordered-disordered interaction parameters $100 \%$

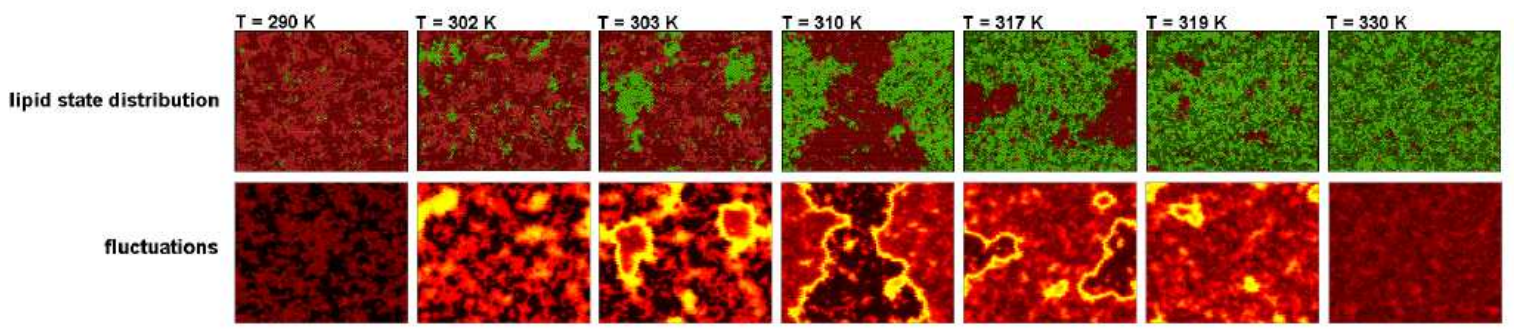

ordered-disordered interaction parameters $90 \%$

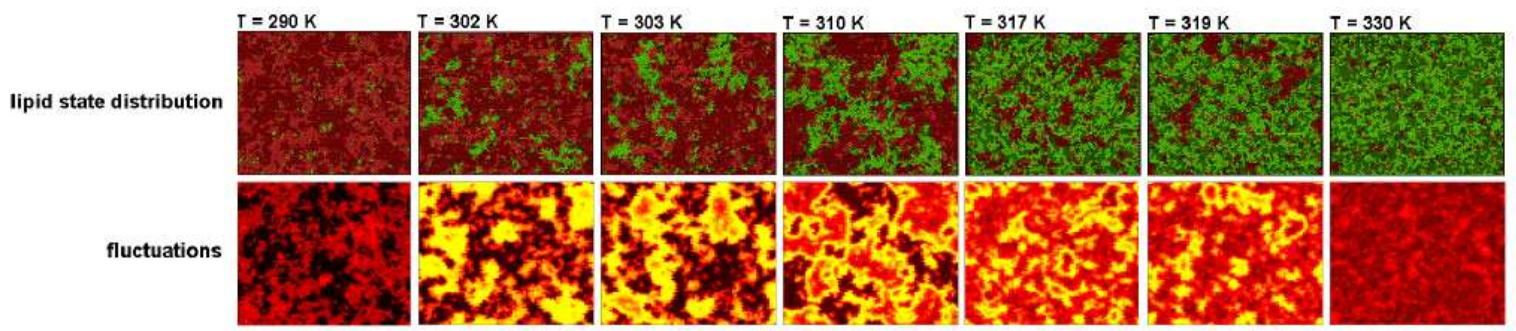

ordered-disordered interaction parameters $80 \%$

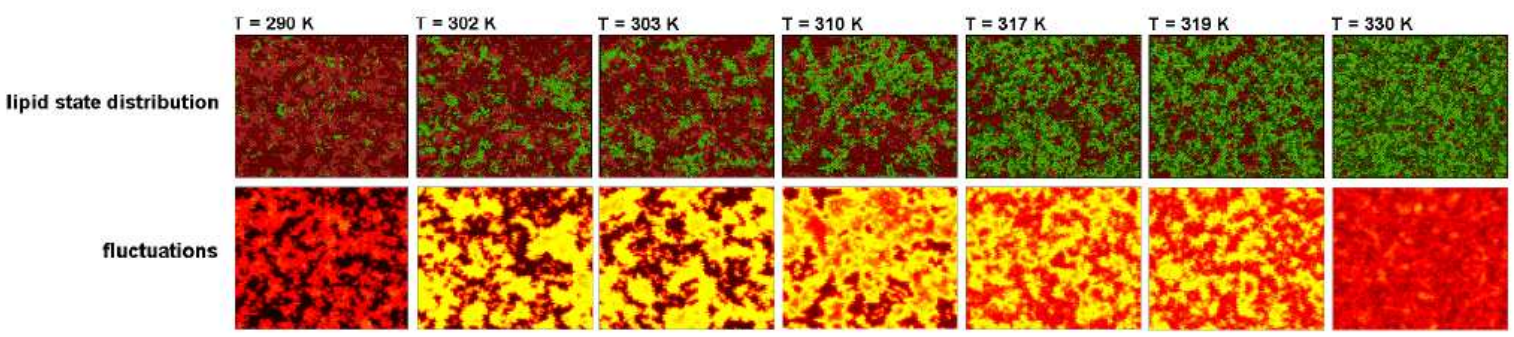

Figure 4.30: Dependence of fluctuations and the lipid chain state distribution on temperature and ordereddisordered interaction parameters. The lipid chain state distribution and the respective fluctuations were obtained from simulations of an equimolar DMPC:DSPC lipid system. The matrix size was set to 80 chains $\times$ 80 chains. Simulations were performed at different temperatures. Interaction parameters between ordered and disordered lipid chains were set to $100 \%$ (top), $90 \%$ (center) and $80 \%$ (bottom) of the original values (see table 3.1). Local fluctuations of a distribution snapshot are plotted below the lipid chain state distribution. In the snapshots of the lipid chain state distribution ordered chains are indicated through red colors, where disordered chains are painted green. The lighter color represents DMPC lipid chains, the darker ones DSPC lipid chains. Large fluctuations are yellow, small ones black and intermediate ones red (Seeger et al., 2005).

bottom) of the original values which are needed to describe the experimentally measured heat capacity profiles (see table 3.1). Changed interaction parameters mimic effects of small molecules like general anesthetics or neurotransmitters (see fig. 4.10). As it is shown in a previous section heat capacity profiles depend on the choice of interaction parameters (see fig. 4.7 and 4.28). Profiles broaden reducing ordered-disordered interaction parameters, unlike-state nearest neighbor contacts become more favorable. Fig. 4.28 shows the simulated heat capacity profiles of the three sets of interaction parameters. At temperatures of 
$302.0 \mathrm{~K}, 303.0 \mathrm{~K}, 317.0 \mathrm{~K}$ and $319.0 \mathrm{~K}$ heat capacity values decrease with reducing the ordered-disordered interaction parameters. This means that fluctuations in enthalpy become smaller. Here, it is important to point out that there is a difference between fluctuations in enthalpy and fluctuations in chain state. The first kind of fluctuation is a macroscopic property of the system, where the second kind of fluctuation (as seen in fig. 4.30) is a local one.

\section{Temperature Dependence and Dependence on Interactions of Ordered and Disordered Lipid Chains}

Macroscopic phase separation can be found in the intermediate temperature regime performing simulations with the ordered-disordered interaction parameter set which is needed for the description of the experimentally determined phase behavior of the DMPC:DSPC system. Only one large domain of ordered and one of disordered lipid chains is present. At temperatures close to the heat capacity maxima $(T=303.2 \mathrm{~K}$ and $T=317.5 \mathrm{~K})$ one finds that one of the two phases dominates and lipid chains of opposite chain state form domains smaller than system size. Small domains can be found in macroscopic domains, in general. These domains are characterized by large fluctuations. It is also found that fluctuations are enhanced at domain boundaries. Fluctuations in domains of disordered lipid chains are larger than in domains of ordered chains. This gets evident from comparing fluctuation snapshots at $290.0 \mathrm{~K}$ and $330.0 \mathrm{~K}$. It should also be noted that the different lipid species do not order randomly in the case that chains are of same state. Domains of different lipid chain species are present.

A reduction in interaction parameters of ordered-disordered contacts leads to an increase of domain boundaries and in total to a different domain pattern. Microscopic phase separation is present. Fluctuations in chain state become enhanced. As mentioned heat capacities differ using the three different sets of ordered-disorder interaction parameters, such do macroscopic enthalpy fluctuations. At temperatures of $302.0 \mathrm{~K}, 303.0 \mathrm{~K}, 317.0 \mathrm{~K}$ and $319.0 \mathrm{~K}$ fluctuations in enthalpy are stronger the higher interaction parameters. Local fluctuations in chain state, however, are increased if ordered-disordered interaction parameters are smaller. This becomes evident from comparing the fluctuation snapshots of fig. 4.30 at the same temperature, but different ordered-disordered interaction parameters.

\section{Dependence of Domain Sizes on System Size}

The impact of changed interaction parameters gets even more evident performing simulations at one temperature, but with different simulation matrix sizes. These were done on a DMPC:DSPC 50:50 mixture for the given three parameter sets at a temperature of $310 \mathrm{~K}$. System sizes were varied to be 30 chains $\times 30$ chains, 60 chains $\times 60$ chains, 120 chains $\times 120$ chains and 180 chains $\times 180$ chains. If one finds macroscopic domains formation an increase of the simulation matrix automatically leads to an increase of the macroscopic domains. They stay on the size of the whole matrix. This also means that the relative proportion of domain boundary decreases. Local fluctuations decrease relatively, since these are enlarged at domain interfaces. Reduced ordered-disordered interaction parameters lead to microscopic phase separation. The domain pattern does not depend on the size of the simulation matrix. Fluctuation properties differ too. Due to a decrease of the interfacial boundaries in the case of macroscopic domain separation one finds that fluctuations do not dominate the system. Lower interaction parameters result in a domain pattern 


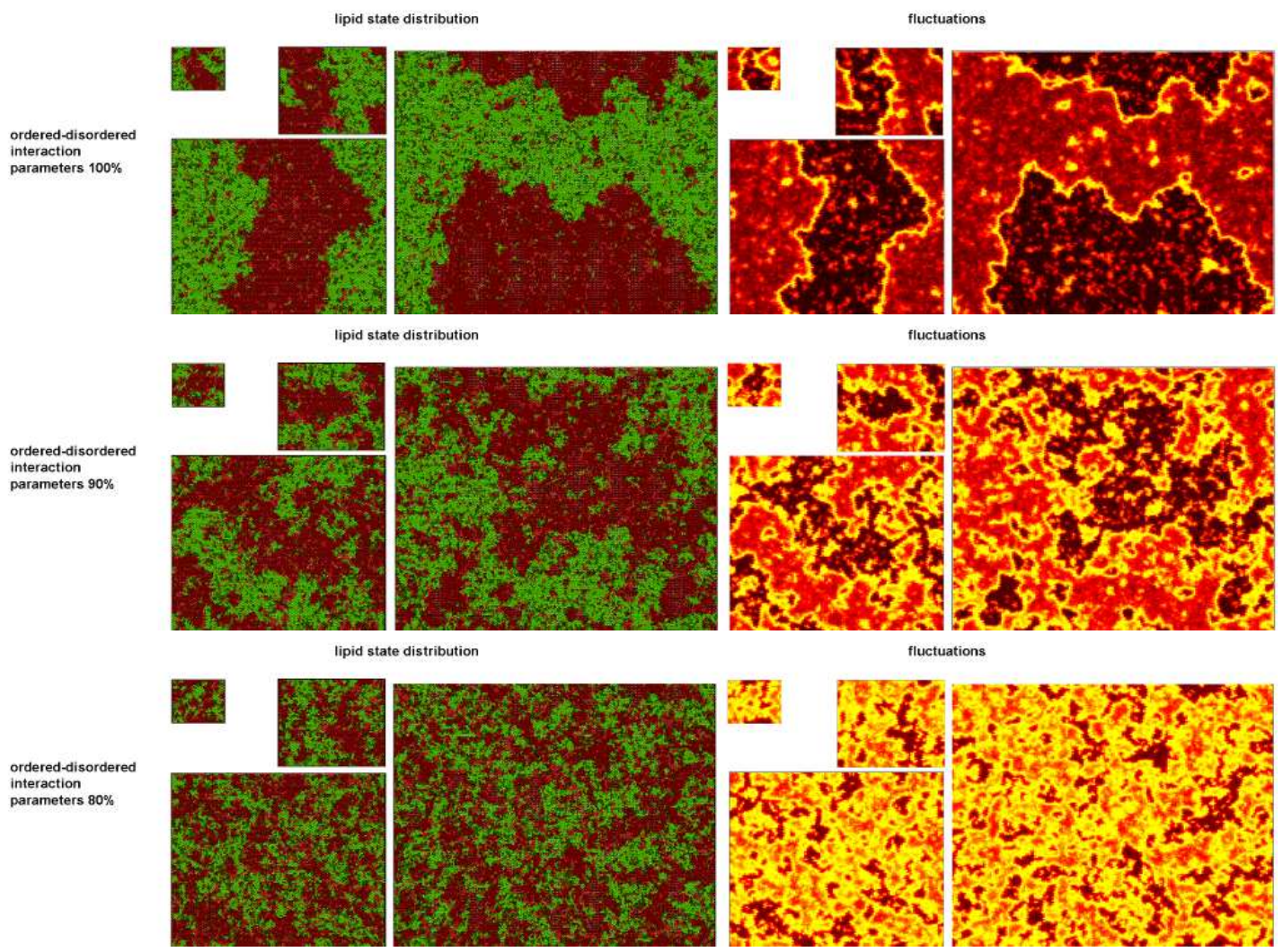

Figure 4.31: Snapshots of the lipid chain state distribution and fluctuations at different matrix sizes $(30$ chains $\times 30$ chains, 60 chains $\times 60$ chains, 120 chains $\times 120$ chains, 180 chains $\times 180$ chains $)$. At a temperature of $310 \mathrm{~K}$ simulations of a DMPC:DSPC $50: 50$ mixture were performed. The left column shows the snapshots of the lipid chain state distribution. Ordered chains are red, disordered ones green. Lighter colors indicate DMPC lipid chains and the darker ones DSPC lipid chains. The belonging fluctuations can be found in the right column. Low fluctuations are drawn black and large ones yellow. The ordered-disordered interaction parameters were varied (100\% (top), $90 \%$ (center) and $80 \%$ (bottom) of the interaction parameters of table 3.1). In the case of high interaction parameters domains grow with matrix size. Macroscopic phase separation gets lost with decreasing ordered-disordered parameters. In the latter case local fluctuations in chain state dominate the matrix (Seeger et al., 2005).

which induces strong local fluctuations which dominate the matrix. This is already obvious for the system with a reduction to $90 \%$ of the interaction parameter values of table 3.1 , it is even clearer with parameters lowered to $80 \%$. In the latter case the whole matrix is dominated by strong local fluctuations due to an almost total loss of large-scale domain formation.

\section{Summary}

Small changes in ordered-disordered interaction parameters have strong implications on the distribution of lipid chain state and local fluctuation properties of lipid membranes. Domain formation happens on different length scales. Macroscopic and microscopic phase separation can be found, dependent on temperature and the interaction of unlike-state lipid chains. Local 
fluctuations are enhanced at domain boundaries. A loss of large scale domains formation leads to a dominance of local fluctuations. Not only domain formation properties describe the physics, but also fluctuations enter the picture.

Domain formation depends on temperature, composition and the addition of small molecules. These processes are interesting since they are related to the diffusion and relaxation behavior, as described in previous sections of this chapter. 


\section{Chapter 5}

\section{Discussion}

The purpose of this thesis was to achieve a deeper understanding of melting transitions and accompanying influences on various physical properties in artificial membranes through both experiments and simulations. The emphasis is put on diffusion, relaxation, domain formation and fluctuation processes. Experimental techniques are mainly based on calorimetry, but also results from FCS are used. The latter ones were performed by A. Hac and described in detail in Hac (2004). A numerical model accompanies the experimental results. Molecules like general anesthetics, neurotransmitters or antibiotics influence the melting behavior of artificial phospholipid membranes and therein affect domain formation, fluctuations and also the diffusion and relaxation behavior.

The model is able to describe and predict measured fluorescence intensity autocorrelation profiles. It has already been part of my master's thesis (Seeger (2002)), but the model went through some further refinements and extensions in the context of this work. Further it is found that diffusion depends on domain formation and time scales and the strength of fluctuations in chain state.

From our experimental and numerical studies it becomes evident that relaxation times are related to macroscopic fluctuations in enthalpy. In the proximity of phase transitions fluctuations in enthalpy enhance and happen on longer time scales. Hence, changes in the melting behavior have an impact on relaxation processes.

Finally, a pure theoretical study is concerned with domain formation on different length scales and fluctuations in chain state. Addition of anesthetics or neurotransmitter lead to a different domain pattern. Macroscopic enthalpy fluctuations diminish, but local fluctuations in chain state become larger. A complete physical picture of domain formation must include domain structure and fluctuations.

In the first part of this chapter the presented results and their relationship is discussed. Next, the relevance of these studies for biological membranes is illustrated. The final section of this thesis deals with suggestions about new research perspectives in the topic of biological membranes and the triggering of the physiology of biomembranes by physical mechanisms.

\subsection{Discussion of Results}

Heterogeneities in biological membranes are believed to be important in biological function (Chapman, 1971; Träuble, 1971; Sackmann, 1984; Brown and London, 1998). In biomembranes different kinds of domains can be induced through processes such as protein aggre- 
gation or lipid domain formation. In this thesis the focus is put on lipid domain formation. Equilibrium and non-equilibrium experimental studies were carried out including diffusion studies, differential scanning calorimetry and pressure perturbation calorimetry. A numerical two state model as introduced by Sugar et al. (1999) was refined for a further evaluation of the experimental data. At first, a discussion of the model's possibility in describing the phase behavior of a two component lipid system and its limitations are given. Then the results about diffusion properties in a two component lipid system are explained and compared with previous studies. The next section deals with the influence of anesthetics, neurotransmitters and antibiotics on melting profiles of phospholipid membranes. Implications on biological function due to a change of the melting behavior are suggested. Then the findings on the relaxation behavior of artificial membranes are discussed and compared to previous studies as they are reviewed in sec. 1.4. Domain sizes, their dependence on order-disorder interaction parameters and accompanying fluctuations are disputed, as well. The discussion of the presented results ends with a short summary, which intends to explain that all investigated processes cannot be seen independently.

\subsubsection{Modeling of Melting Processes in a Two Component Lipid System}

Sugar et al. (1999) introduced a two state model of chain state in order to describe the phase behavior of a DMPC:DSPC membrane system. Lipid chains are arranged on a hexagonal lattice and just nearest neighbor interactions are accounted. Rotations of neighboring lipids and lipid exchange of neighboring or distant (non-physical exchange) lipids are incorporated. Sugar et al. (1999) also used the model for the analysis of geometrical properties of clustering of ordered and disordered chains (Sugar et al., 2001). We have refined the model by limiting lipid chain exchange to the swapping of neighboring lipids. Our model also includes differences of the rate of diffusion in the "so" and "ld" phase. The model allows the calculation of heat capacities. Changes in the simulation details, like another definition of the Monte Carlo cycle, must not lead to other results in heat capacity, since this would mean a violation of detailed balance. It was verified that various definitions of the Monte Carlo cycle lead to the same heat capacity profiles.

\section{Considerations about Sources of Error}

The model requires the determination of ten parameters which just depend on the used lipid species and must not differ using different lipid compositions. All parameters can be estimated directly or indirectly from measured heat capacity profiles. A comparison of experimental and simulated heat capacity profiles shows that they agree. Small deviations can be monitored at the low temperature and high temperature regime of the transition and in the regime of phase coexistence. In the latter case calculated heat capacity values are always slightly lower than the measured ones. In the first case one finds that in the simulation melting processes start at lower temperatures and end at higher temperatures. This is indicated through a smoother increase of simulated heat capacity profiles. Still using tangents on the lower and upper temperature limits of the $\Delta c_{p}$-profiles one finds that the two determined phase diagrams agree well.

Inconsistencies among of heat capacity profiles and phase diagrams can be explained by the strong simplified model, the limitation in determining a perfect set of parameters and experimental errors. Experimental errors can be due to uncertainties in the weighting process, 
the mixing process of the two lipid solutions when dissolved in organic solvent and the addition of the buffer solution to the dried lipids. This again means that the parameters applied in the simulations are inexact. Better accuracy of the parameters used would lead to a better description. Limits of the model are the strong simplification using a two state model of chain state, arranging lipid chains on a lattice and neglection of topological effects like the formation of the ripple phase. In suspensions of multilamellar vesicles one finds a pre-transition from the "so" to the ripple phase or vice versa. In the ripple phase line defects of disordered lipids are present. This transition happens at a temperature well below the one of the main phase transition.

Limiting the number of chain states to an ordered or disordered configuration does not correspond to the experimental situation. Simulations with ten states of chain order do not show any advantage in the description of macroscopic properties like heat capacity or macroscopic fluctuations. They are favorable in studies describing microscopic properties (Pink et al., 1980).

The used model is based on arranging lipid chains on a hexagonal lattice structure. It is, however, well known that lipid membranes lose their lateral order throughout a melting process (Janiak et al., 1979).

Determined experimental heat capacity profiles are measured on multilamellar vesicles, which show besides the main phase transition a pre-transition at lower temperatures. Heimburg (2000) has presented a model which includes coupling of the two monolayers of a bilayer and curvature. With this model the pre-transition, as well as the main transition are successfully described. Our model is not sufficient for a description of the pre-transition.

Even though the model has considerable simplifications, it is still able to describe the phase behavior of a two component lipid system in our case a DMPC:DSPC lipid system. Therefore, it seems that it is valid to use it in studying diffusion, relaxation and fluctuation properties.

\subsubsection{Diffusion in Planar Membranes}

Experimental determined FCS autocorrelation profiles (measured by A. Hac; see Hac (2004)) are well predicted by the introduced minimalistic numerical model. Deviations are due to experimental problems like the scattering in gained autocorrelation curves at the same lipid composition and temperature (see fig. 4.3) or due to the simplified model. The emphasis of this thesis is the modeling of the diffusion processes. Therefore, further on the experimental problems are not discussed and the reader is referred to Hac (2004).

\section{The Model}

The modeling of diffusion processes is based on the exchange or a rotational movement of two neighboring lipids. In the first case four lipid chains are involved, in the second two. A microviscosity is introduced which models the difference between diffusion in a "so" and "ld" membrane. At domain boundaries lipids have an environment of ordered and disordered lipids. Therefore, it is assumed that lipid exchange rates display an intermediate behavior at domain interfaces. At these elastic constants might be increased because of strong fluctuations (Ivanova et al., 2003). This may lead to defect formation and diffusion because of a defect mechanism. There is, however, a lack in experimental data, so this possibility is not considered in our model.

Chains are arranged on a hexagonal lattice at all temperatures. This is not true in an 
experimental situation since melting of a lipid membrane includes melting of the lipid and the lattice structure (Janiak et al., 1979). The melting of the lattice structure, however, is reflected in the choice of the diffusion barrier as introduced in eq. 3.16.

A further problem is that the experimental diffusion studies were performed on stacks of bilayers which form a ripple phase. A pre-transition from the "so" to ripple phase happens several degrees below the main phase transition. The latter is characterized through the formation of line defects of disordered lipids (Heimburg, 2000). It has been suggested that diffusion in an artificial membrane in the ripple phase is along defects (Schneider et al., 1983). The used model, however, cannot describe the ripple phase. The experimental results might be influenced by diffusion along line defects. In the model this again is depicted through the choice of the activation barrier.

All this suggests that the model is a strong simplification which might turn out to be oversimplified in the long run. It fails in the microscopic description. The strength of our MC simulation lies in the description of macroscopic properties, as it is successfully shown in this study.

\section{Comparison to Previous Studies}

In the past mainly phenomenological free volume theories have been used for the description of lipid diffusion (Galla et al., 1979; Vaz et al., 1985; Clegg and Vaz, 1985). In recent years Molecular Dynamics simulations have become popular (Böckmann et al., 2003; Falck et al., 2004). The latter method is based on first principles, but simulation times are limited to roughly $100 \mathrm{~ns}$. Studying diffusion processes in this time regime has to be regarded as not sufficient if no direct comparisons to experiments can be made. Sugar and Biltonen (2005) have used the same model as we, but they varied the size of a diffusion step based on free volume considerations. Our model using a phenomenological microviscosity and the free volume approach can be translated into each other. E.g. in the case of diffusion in a "so" phase, the free volume is small and the diffusion rate in our simulation is decreased. Neither of the two approaches can be favored over the other. Both models, however, have presently an advantage over Molecular Dynamics simulations, because of their possibility to describing diffusion processes over longer time scales.

The introduced model might be an oversimplification. Still, it is successful predicting experimental fluorescence intensity autocorrelation profiles (see fig. 4.2). This allows us to believe that additional results from simulations could be valid for the experimental system. One objection might be that the simulated and the experimental systems are of different size. As it is shown in fig. 4.4 the autocorrelation curves from simulations do probably not depend on the choice of matrix size, and if they do the effect is minor. Deviations of the profiles simulated with different matrix sizes can be explained by a lack of diffusion data especially in the case of the biggest matrix size.

\section{Timescales in the Simulation}

Monte Carlo simulations allow us to change the timescales of fluctuations in state change and diffusion steps. It is shown that different timescales influence fluorescence intensity autocorrelation profiles (see fig. 4.5). Due to the scattering in the experimental data we are not able to determine the most probable ratio between diffusion and relaxation time scales, even though it is hidden in the experimental curves. Superposition of diffusion processes in 
the "so" and "ld" phases fails in describing measured autocorrelation profiles in the case of ordered/disordered domain coexistence (see fig. 4.5). This suggests that experimental profiles contain information which is not reflected in the superposition of diffusion processes in the pure phases. We suggest that this is because the diffusion properties are influenced by fluctuations in chain state.

Korlach et al. (1999) measured diffusion processes in LUVs of different DLPC:DPPC compositions with FCS. They evaluated their data by fitting one or two diffusion processes. They assumed that there might be a fast and a slow diffusing component. Interestingly, the fast diffusion constants differed when diffusing in a pure "ld" membrane or in the phase coexistence regime. These results were verified by studies on supported stacks of DLPC:DPPC and DMPC:DSPC bilayers (Hac, 2004). In the latter case it was found that in the coexistence regime diffusion of the slower diffusing component is faster in comparison to diffusion in the pure "so" phase. Applying the same fit algorithm using two diffusing components to our simulated fluorescence intensity autocorrelation profiles reveals that diffusion times of the slow and fast diffusion component depend on the timescales of fluctuations in chain state and diffusion steps. They do not equal diffusion times in the pure phases. As in the experimental systems, diffusion of the slow component is faster and the diffusion of the fast component becomes slower. These results question the validity of interpreting autocorrelation profiles of measurements in the phase coexistence regime purely by means of fast and slow diffusion component. Instead, autocorrelation curves depend on the time scales of state changes and diffusion steps.

Measured and simulated autocorrelation profiles can also be fitted applying a fit with an anomalous diffusion term (Schwille et al., 1999). In our study, simulated profiles are equally well fitted using two diffusing components or anomalous diffusion. The ratio between chain state changes and diffusion steps influences also the diffusion time $\tau$ and the exponent $\alpha$. The diffusion time and the exponent decrease with a slower rate of chain state changes. It is also explained that the choice of order-disorder interaction parameters influences the mean diffusion time and the exponent $\alpha$ (see fig. 4.8). In the domain coexistence region it is found that on the length scale of the focus the anomalous diffusion coefficient increases with decreasing interaction parameters, so in some cases diffusion even gets normal. This can be understood as being due to the labeled chain's fluctuations in chain state during the time in the focus. With smaller interaction parameters local fluctuations in chain state dominate the membrane system (see fig. 4.30 and fig. 4.31). Calculating averaged chain states one finds that on average the whole matrix is occupied by chains of almost the same chain order and the average system is close to being homogenous (data not shown). In other cases, with bigger interaction parameters, the distribution of averaged chain states shows a higher degree of heterogeneity (data not shown) since local fluctuations are not dominant. They are enhanced at domain interfaces and small domains also show strong local fluctuations. The same is true for comparing results from different ratios of chain state changes to diffusion steps. With slower local fluctuations and longer domain life times diffusing lipids experience more hindrances. Again, this means that measured autocorrelation profiles give indirectly information about local fluctuations of the lipid membrane. A finding about underlying physical processes which cannot be found by only using phenomenological fit procedures, assuming a fast and a slow diffusing component or anomalous diffusion. With the help of our simulations we are able to interpret the experimental autocorrelation profiles in their full complexity.

Theoretical studies about anomalous diffusion in biological membranes were mainly done 
by M. Saxton. Hard-core lattice models were used. Different kinds of obstacles were defined and the influence on diffusion properties could be studied. The pure presence of obstacles might induce anomalous diffusion (Saxton, 1994), but also binding influences diffusion properties (Saxton, 1996). A shortcoming of Saxton's works, however, is that they are not dependent on temperature and that they do not depict situations in a real membrane. This is the strength of the presented model. It describes thermodynamical averages of artificial lipid membranes correctly and heterogeneities arise from the physics of the lipid membrane. Before the publication of parts of this study (Hac et al., 2005) one theoretical paper about diffusion in a lipid/sterol system based on thermodynamics information was published (Polson et al., 2001). Recently, Sugar and Biltonen (2005) used a similar model. Our study, however, has the advantage of direct comparison to experiments.

\section{Summary and Conclusion}

The strength of this study lies in a combination of numerical and experimental ${ }^{1}$ results. Needed parameters for the description of the phase behavior are taken from calorimetric experiments. Differences between diffusion in the pure phases are considered through introducing a microviscosity. The parameter which is needed for implementing differences of diffusion in "so" and "ld" membranes is again obtained from a comparison to FCS experiments. Our Monte Carlo simulations can predict the expected experimental diffusion behavior. Simulated and measured profiles agree well. This allows us to gain information from the simulations and from these conclusions about the physics of the experimental artificial membrane system can be drawn. It is found that fluctuations have a great influence on the diffusion behavior of two component lipid membranes. It is also shown that one has to be careful with applying phenomenological fit routines to the analysis of experimental fluorescence intensity autocorrelation curves, since they do not necessarily reveal a deep insight on the underlying physical mechanisms.

\subsubsection{Melting Profiles of Artificial Membranes}

Phospholipid membranes display a melting transition from a highly ordered to a highly disordered state at physiological relevant temperatures. Details like the transition midpoint and the cooperativity of the transition depend on e.g. the kind of lipid, chain length, degree of saturation, vesicle type, $\mathrm{pH}$, ion concentration and pressure. Addition of non-lipid molecules influence the melting behavior. In this thesis it is shown that general anesthetics (1-octanol, halothane), neurotransmitters (serotonin) and peptides (gramicidin A) shift the temperature regime towards smaller temperatures and broaden the profile.

\section{The Antibiotic Gramicidin A}

Gramicidin A is a channel forming peptide which acts as an antibiotic. From its influence on the melting behavior one can deduce that gramicidin A clusters and fluctuations increase in the vicinity of the peptide clusters (Ivanova et al., 2003). It decreases the permeability of phospholipid towards the fluorescence marker rhodamine (Makarov, 2005). It is, however, known as forming cation specific pores (Gennis, 1989).

${ }^{1}$ The experimental work on diffusion properties was performed by A. Hac (Hac, 2004). 


\section{The Neurotransmitter Serotonin}

Here, the influence of the neurotransmitter serotonin on the melting behavior was presented. The same behavior is found for other neurotransmitters (Pollakowski, 2003). The general consensus of the action of neurotransmitters is that they act on proteins. In some diseases like schizophrenia the phospholipid metabolism is perturbed (Peet et al., 1999). This suggests that not just proteins, but also the lipid membrane needs to be considered explaining the function of neuronal systems. Pollakowski (2003) speculates that neurotransmitter change the state of neuronal membranes and act indirectly on channel protein activity.

\section{The General Anesthetics 1-Octanol and Halothane}

Anesthetics like 1-octanol and halothane influence the melting behavior of phospholipid membranes. In this study it has been verified on DMPC membranes. Mengel and Christiansen (2005) have analyzed the behavior of the same alcohols on the melting behavior of DPPC lipid vesicles. They could relate the shift in melting temperature and clinical concentrations with each other. Alcohols do not completely dissolve in the lipid membrane, but partition in the lipid and the water phase. 1-octanol partitions stronger in the lipid membrane than halothane. The clinical concentration of halothane is about a factor of 4 higher. In their experiments Mengel and Christiansen (2005) needed about a 7 times higher concentration of halothane to yield the same shift in melting temperature. It has already been shown that the higher the anesthetic potency of solutes the more they shift the melting temperature (Kharakoz, 2001). Heimburg and Jackson (2006) try to relate a recently published theory on soliton propagation in nerve membranes (Heimburg and Jackson, 2005) and the decrease of melting temperature with general anesthesia. In their view a nerve pulse is a soliton. They claim that a reduction in melting temperature means an increase of the threshold of soliton propagation. The theory by Heimburg and Jackson challenges the accepted one of Hodgkin and Huxley (1952).

Knowing the partition coefficient of 1-octanol in DPPC $(P=200$; see (Jain and Wray, 1978)) they can calculate the molar ratio of 1-octanol in the lipid membrane. They can determine the shift in transition temperature using:

$$
\Delta T_{m}=-\frac{R T_{m}^{2}}{\Delta H_{0}} x
$$

with $\Delta T_{m}$ the change in melting temperature, $T_{m}$ the melting temperature, $R$ the gas constant, $\Delta H_{0}$ the transition enthalpy and $x$ the molar ratio of the anesthetic. We can perform the same analysis, since the partition coefficient of 1-octanol in DMPC membranes to our knowledge has not been determined. Taking the same partition coefficient as the one for DPPC membranes, calculating with it the concentration of 1-octanol in the membrane and applying the whole to eq. 5.1 one estimates the expected shift of the melting temperature. The obtained values are, however, in all cases higher than the measured shifts. From theoretical considerations on the partition of ethanol in binary lipid mixtures it is known that alcohols partition differently well in different lipid environments (Cantor, 2001a). It should be noted that partition coefficients increase in the transition regime with a maximum at the transition midpoint (Trandum et al., 1999). For a final discussion the determination 
of the partition coefficient is needed.

\section{Summary and Conclusion}

Interestingly, all three groups of molecules (anesthetics, neurotransmitters, antibiotics) influence the melting behavior of lipid membranes. In this way, they also change other physical properties like diffusion properties, relaxation behavior, domain structure and local and macroscopic fluctuation properties. This e.g. influences permeability (Makarov, 2005) or elastic constants (Heimburg, 1998), which are coupled to fluctuations.

\subsubsection{Relaxation Time Measurements}

Pressure perturbation calorimetry is used to study relaxation phenomena of domain formation processes in artificial membranes. Systems range from one component lipid systems of different vesicle type to one component lipid systems with addition of anesthetics, neurotransmitters or antibiotics. Relaxation processes are monitored by measuring the calorimeter response of the VP-DSC or following the temperature course of the sample solution after a change in pressure. The first method gives a time resolution of about $3-4 s$ and the second method one below $500 \mathrm{~ms}$.

All measurements reflect a linear relation between relaxation times and the respective excess heat capacities (see figs. 4.15, 4.17, 4.18, 4.19 and 4.21). This is an interesting finding since membranes of different lipid species melt at different temperatures, the half width and melting temperature of the transition depends on the type of vesicle and on addition of other non-phospholipid molecules. Through Monte Carlo simulations and a non-equilibrium thermodynamics theory one can relate these relaxation times to cooperative fluctuations in enthalpy (Grabitz et al., 2002). Relaxation times are a function of the final temperature.

\section{Evaluation of the Calorimeter Response}

As mentioned method one uses the response of a calorimeter to keep the temperature constant after a change in pressure. The strength of the lipid signal depends on the point of transition one jumps to and the lipid concentration. The latter one needs to be high enough so that the lipid signal is strong enough in comparison to the perturbation signal, which arises from the setup. On the other hand it should not be too high. This is because the calorimeter needs to compensate the heat release or absorption. If it is too high the calorimeter fails in its compensation and the temperature cannot be kept constant. This is especially a problem after positive pressure jumps (ppjs), when the calorimeter needs to cool which is slow.

The heat compensation of the calorimeter is not perfect and after a pressure jump the sample's temperature changes, which again is compensated with time. The phase behavior at the maximal and minimal temperature are different. How much it differs depends on the difference between the maximal and minimal temperature and the cooperativity of the melting transition. At temperatures in between the both extremes the phase behavior might again vary. If temperature changes are small these differences are, because of the fluctuations of the system, not distinguishable. The calorimeter response can be analyzed in this case. This is different when temperature changes are big. Then the system is not in a defined state. The relaxation behavior of the system changes with time and the measured relaxation process reflects a superposition. Whether a temperature change has to be seen as being big 
or small depends on the system. In the case of very cooperative melting transitions (like for MLVs) with transition half widths of around $0.1 \mathrm{~K}$ the temperature change must be kept smaller than this. For LUVs with transition half widths of several degrees a difference of $0.1 \mathrm{~K}$ would not have a strong influence on the phase state. High lipid concentrations enhance this problem. Performing npjs one finds that problems mainly occur at higher temperatures, whereas it is the lower temperature regime for ppjs. One can use results from both jump directions, so that they complement each other.

\section{Temperature Corrections}

Temperatures used for ppjs need to be corrected, since melting temperatures under higher pressures increase. Even the temperatures from npjs have to be corrected by $-0.01 K$, if compared to a $\Delta c_{p}$-profile measured at $5 \frac{K}{h}$. This might lie in hysteresis effects (see fig. 4.13). Excess heat capacity profiles depend on scan direction and scan rate. Fast pressure changes lead to fast changes, which might be reflected by a heat capacity curve measured with higher scan rates. Melting profiles from scans with high scan rates shift profiles to lower temperatures. In this context, it is, however, puzzling why relaxation time profiles measured with ppjs need to be shifted by $0.90 K$ and not $0.84 K$ as expected from the previous arguments. This is not understood at this point. The same shifts need to be used for results gained with method II, where the temperature development of the lipid solution is evaluated.

\section{Broadening of Heat Capacity Profiles}

In some cases it seems that the relaxation time profile is broadened in comparison to the excess heat capacity curve. This can be understood with a broadening of the respective transition profile during the experiments. A series of relaxation time measurements were performed on successive days. Comparison of $\Delta c_{p}$-profiles which were measured on different days sometimes showed a broadening of the heat capacity profile with time. This also means an altering of the relaxation behavior. Already Grabitz et al. (2002) reported about a measurement were they measured different relaxation times on the same sample. They claimed that this arose from a swelling of the sample and therefore a change in melting behavior. They, however, did not do another DSC-scan since the effect was discovered too late. Their sample was kept in the fridge for a week between the two different measurements. Therefore, our samples were kept in the fridge for a few days after preparation. DSC-scans, however, often resulted in the same profile independent of how long the sample was kept in the fridge.

One possible reason for a broadening might be contaminations of the pressure cell or the sample itself. Impurities usually lead to a broadening and in a shift in melting temperature. Shifts in temperature were usually not found. If a contamination of the lipid sample was the reason for the broadening, still the influence of the melting behavior because of the addition of anesthetics, neurotransmitters or antibiotics was dominating. Therefore, the change in relaxation behavior is attributed to mainly the anesthetics, neurotransmitters or antibiotics.

Another putative reason might have been a swelling of the vesicles during the measurements which were performed on several days.

Comparing relaxation times and heat capacity curves one notes that stronger deviations of the profiles usually occur at the upper and lower temperature limits of the transition. One reason might be a possible broadening of the transition profile. This would be especially evident at temperatures where fast relaxation processes are expected. The limited time 
resolution is another explanation.

Broadening of the heat capacity profile can also explain why in some cases phenomenological constants strongly deviated from other measurements since broadening also means a reduction in the maximal relaxation time. Comparing measured relaxation times to the wrong heat capacity profile automatically means a wrong determination of the phenomenological constant.

\section{Faster Relaxation Process}

Comparison of the areas of the calorimeter response at different temperatures verifies that the area is proportional to the released transition enthalpy (see fig. 4.14). This is also true for the area of the lipid and of the perturbation signal (see fig. 4.20). Since the perturbation signal displays a proportionality to the transition enthalpy, there must be a contribution of the lipid in the perturbation signal. We can conclude that the lipid membrane also displays a faster relaxation process which happens on a time scale below the second regime. The contribution of these fast processes is small in comparison to the one of the slow relaxation. As discussed below faster relaxation processes have been reported in the literature.

\section{Evaluation of Temperature vs. Time Curves}

Results from analyzing the temperature course of the sample solution (method II) have not revealed faster relaxation processes either. All curves could be described by one relaxation time. This does not mean that there are no more processes. The findings of the evaluation of the calorimeter response suggest that the contribution of the fast process is small. Therefore, it might lead to a temperature change which is not resolvable with our method.

In our evaluation only results after a pressure increase were used. The choice of lipid concentration is crucial. Concentrations need to be high enough so that relaxation processes can be detected at higher temperatures where the heat release from the lipids is small. If concentrations are too high a strong lipid signal might lead to temperature changes which perturb the analysis. This problem gets evident when jumping through a major part of the transition.

As well the choice of pressure change depends on two considerations. The first one is that bigger changes in pressure result in a stronger perturbation signal. This means that the contribution of the lipid signal decreases relatively to the perturbation signal with increasing pressure change. If the pressure change is not high enough heat capacity profiles measured with and without additional pressure might intersect. Therefore, the lipid contribution decreases again at lower temperatures. In the case of LUVs pressure changes were $+40 \mathrm{bar}$ and lipid concentrations $40 \mathrm{mM}$. For the measurement on the DMPC MLVs the concentration was set to $20 \mathrm{mM}$ and the pressure was increased with $15 \mathrm{bar}$. Because of the small half width of the transition of MLVs already a pressure of 15 bar is enough so that the two profiles do not intersect. In the case of LUVs profiles still intersect if the pressure difference is $40 \mathrm{bar}$. It was controlled that relaxation times do not depend on the choice of amplitude of the pressure change.

The time resolution using npjs is not as good as the one of ppjs. The reason is that the pressure release takes about a factor of 2.5 longer than the one of pressure increase. Therefore, just ppjs were performed and analyzed. Results gained from the two different pressure change directions cannot be used for complementing each other as in the case of the 
previous method (method I). Often there is a lack in the number of measurement points at lower and higher temperatures. Still, one can conclude a proportionality between relaxation times and excess heat capacity curves.

\section{Comparison Method I and Method II}

With pressure perturbation experiments on solutions of MLVs of DMPC using method I and method II it was controlled that both methods reflect the same behavior. In the two cases relaxation times at the transition midpoint differ. Using method I the relaxation time was measured to be $31.0 \mathrm{~s}$ and $25.2 \mathrm{~s}$ with method II. The heat capacity values at the transition midpoint, however, vary and the two profiles show different broadening. The deviation is small. The phenomenological constants agree with each other. Using method II it was determined to be $L=6.8 \cdot 10^{8} \frac{\mathrm{JK}}{\mathrm{mols}}$. The other analysis using method I it was calculated as $L=6.6 \cdot 10^{8} \frac{\mathrm{JK}}{\mathrm{mols}}$. Since the gained phenomenological constants should equal rather than the absolute relaxation times it can be concluded that the two different evaluation methods lead to the same results.

\section{Linearity of Relaxation Times and Heat Capacity}

Plotting relaxation times against heat capacity (see fig. 4.22) we found a linear relationship between relaxation times and heat capacity. Linear fits do not intersect the origin. We argue that this behavior arises presumably because of the time resolution limit of the two methods applied. With evaluating the calorimeter response we cannot detect relaxation processes faster than about $3-4 s$. Using the temperature course of the sample solution we can obtain relaxation times down to $300-400 \mathrm{~ms}$. We do not attribute the deviation from not intersecting with the origin to any physical characteristic of the artificial membrane systems.

\section{Phenomenological Constants}

All estimated phenomenological constants are displayed in table 4.1. There is no theoretical justification, but the calculated phenomenological constants suggest that they are the same for MLV suspensions. The average value is $6.7 \pm 0.7 \cdot 10^{8} \frac{\mathrm{JK}}{\mathrm{mol}}$. Especially two measurements deviate strongly from the average. This possibly occurred because of a broadening of the melting transition profile during the experiments. A wrong maximal value in heat capacity was presumably taken as a reference to the relaxation time measurements.

Phenomenological constants gained from measurements on the LUV suspensions also seem to be constant $\left(\langle L\rangle=18.0 \pm 2.2 \cdot 10^{8} \frac{\mathrm{JK}}{\mathrm{mol} s}\right)$. It is not as evident as in the case of MLVs. More measurements are needed to verify this finding. Phenomenological constants are, however, different to the ones of the MLV systems.

Already Grabitz et al. (2002) suggested that phenomenological constants are the same for all systems, even biological membranes. Our findings support this suggestion in the sense that they are constant when considering the same vesicle system. They seem to differ between LUVs and MLVs.

In our experiment the transition enthalpy of our experiments on multilamellar DMPC vesicles was measured to be $21.5 \frac{\mathrm{kJ}}{\mathrm{mol}}$. In the literature this value is given as $(25 \pm 10) \frac{\mathrm{kJ}}{\mathrm{mol}}$ (Koynova and Caffrey, 1998). Taking this value phenomenological constants of the DMPC MLV systems are $20 \%$ higher. With this higher transition enthalpy we would calculate a 
phenomenological constant of about $8.0 \cdot 10^{8} \frac{\mathrm{JK}}{\mathrm{mols}}$. Grabitz et al. (2002) report a phenomenological constant of $7.4 \cdot 10^{8} \frac{\mathrm{JK}}{\mathrm{mols}}$ for a suspension of DMPC MLVs and $8.5 \cdot 10^{8} \frac{\mathrm{JK}}{\mathrm{mols}}$ for multilamellar DPPC vesicles. It needs to be noted that Grabitz et al. (2002) compared their relaxation data to heat capacity profiles scanned at a rate of $1 \frac{K}{h}$. Ours were measured with a scan rate of $5 \frac{K}{h}$. In general heat capacity profiles measured with different scan rates differ in half width, maximum heat capacity and transition midpoint temperature.

They also measured relaxation times in a DPPC LUV suspension. Relaxation times were as high as $3 s$, which is in the resolution limit and the measurement has to be taken with care. From this they calculated a phenomenological constant of $13.9 \cdot 10^{8} \frac{\mathrm{JK}}{\mathrm{mols}}$. This is in the range of our findings too.

There are deviations but all in all our results and the ones of Grabitz et al. (2002) agree. New is that addition of small molecules or peptides alters the relaxation behavior, but heat capacity and relaxation times are still linear. This is a non-trivial finding.

Grabitz and collaborators estimated that relaxation times of biological membranes lie in the $10-100 \mathrm{~ms}$ regime ${ }^{2}$. This estimation stays true even if one takes a higher phenomenological constant as a reference as it is found in suspensions of large unilamellar vesicles. This is an important conclusion since a time scale of $10-100 \mathrm{~ms}$ is of physiological relevance.

\section{Degree of Perturbation}

One critics on the studies of Grabitz et al. (2002) and ours is that in the non-equilibrium theory fluctuations in enthalpy around an average are considered. Jumps from the pure membrane phases into a phase coexistence regime, however, induce rather big changes in enthalpy. One might doubt that time scales of enthalpy fluctuations and from pressure perturbation experiments differ. Temperature jump experiments simulations verified that time scales of enthalpy fluctuations probably are the ones which are measured in our pressure perturbation experiments.

\section{Comparison to Previous Studies}

As mentioned in sec. 1.4 relaxation times have been reported in the literature from the $n s$ to hour regime. Most of the results suggest that relaxation processes slow down in the melting regime. The work by Yager and Peticolas (1982) states that relaxation times increase with increasing temperature in the case of melting and increase with lowering temperature in the case of freezing. Since this study is an exception, it is not considered in the following. It seems more important to discuss why others usually report about faster relaxation processes and in two cases slower processes than they are revealed in our experiments. One class of experiments uses perturbations by pressure or temperature change and detection by optical means using fluorescence markers or turbidity. The first one presenting results of temperature jump experiments was Träuble (1971). Sonicated ${ }^{3}$ DPPC lipid vesicles with and without cholesterol were used as model systems. Relaxation times were between $100 \mathrm{~ms}$ and $1200 \mathrm{~ms}$. Turbidity vs. time curves were fitted with just one relaxation process. Cholesterol led to

\footnotetext{
${ }^{2}$ This estimation was gained from a heat capacity profile of lung surfactant. Strictly speaking, one needs to know the exact $\Delta c_{p}$-profile of each biomembrane if one wants to estimate the expected relaxation times of a biological membrane.

${ }^{3}$ Sonification of MLVs leads to a rupture of the vesicles and the formation of small unilamellar vesicles (typically $15-30 \mathrm{~nm}$ in diameter). These ones are unstable and fuse to form LUVs with time.
} 
faster relaxation processes. Cholesterol shifts the melting temperature and broadens the transition profile. Therefore, relaxation times should decrease if seen with respect to our findings. This was also verified by Grabitz et al. (2002). The findings of Träuble (1971) agree with ours qualitatively, since suspensions of sonicated vesicles display a broader transition than solutions of MLVs or LUVs. With time they fuse to form LUVs. From our studies we would expect relaxation times in the regime as measured by Träuble (1971).

Tsong and collaborators (Tsong, 1974; Tsong and Kanehisa, 1977) using the same method as Träuble (1971) gained different results. They described their turbidity measurements with two relaxation processes. The faster relaxation time on unsonicated samples is in the $10 \mathrm{~ms}$ regime, the slower one in the several $100 \mathrm{~ms}$-regime. Both display maxima at the transition midpoint. For a solution of DMPC they determined relaxation times up to $2 \mathrm{~s}$. Relaxation times on DPPC membranes were measured to be faster. Experiments on sonicated vesicles revealed faster relaxation times than in the case of unsonicated ones. From our point of view it is surprising that they found that relaxation times of DMPC are higher than the ones of DPPC. It is expected that sonicated vesicles display faster relaxation processes than unsonicated ones. As already mentioned this is because of the lower cooperativity of the transition. Our experiments have shown that there probably are faster relaxation processes, which means that we cannot rule out that there are processes in the time regime measured by Tsong and collaborators. Still, one would expect that also slower processes should be measurable, as it was reported by Träuble (1971). There are about 30 years between Tsong's and our measurements. In a time range of 30 years purities of lipid samples might have improved. Lipids used in our experiments have a degree of purification of $>99 \%$. For the ones used by Tsong and collaborators no information is available. In the case of MLVs already small impurities might lead to a broadening of the melting curve and therewith shorter relaxation times. Another reason might be the need to implement a window into the setup so that an optical detection is possible. It might act as a heat sink and the sample temperature is not well defined. Membranes which display a very cooperative melting behavior need a very precise temperature adjustment. This is why possible heat sinks might perturb the measurements. However, the same arguments are true for the measurements of Träuble (1971).

A possible temperature inaccuracy is also true for measurements of the groups of Blume and Gruenewald (Gruenewald et al., 1980; Gruenewald, 1982; Elamrani and Blume, 1983; Blume and Hillmann, 1986). They detected kinetics using turbidity or fluorescence anisotropy. They report up to three relaxation processes on various phospholipid systems. They are usually faster than the ones found in our experiments. In one case using a phosphatic acid they found relaxation times as high as $3 \mathrm{~s}$. In most cases, however, they were measured to be in the $1-10 \mathrm{~ms}, 10-100 \mathrm{~ms}$ and $100-1000 \mathrm{~ms}$ regime. The exact values depend on the used lipid species. Another study (Genz and Holzwarth, 1986) using fluorescence techniques report again three relaxation processes. Based on results by Holzwarth and Rys (1984), who claim the occurrence of five relaxation processes, (Genz and Holzwarth, 1986) assume that there are two more relaxation processes which they cannot resolve. The maximal relaxation times of DPPC solutions are smaller than $20 \mu \mathrm{s}, 2.5 \mathrm{~ms}$ and $20 \mathrm{~ms}$. Cholesterol influences the relaxation behavior (Blume and Hillmann, 1986; Genz et al., 1986). Whereas, the first study claims that all three relaxation times decrease with cholesterol content, the second one concludes that one relaxation process becomes faster, one stays constant and the third one slows down. It is not quite clear where these ambiguities come from. That absolute relaxation time values differ is not surprising. In the context of Grabitz et al. (2002) and our study it is evident that changes in the details of the experiments like sample preparation or the choice 
of lipid species have a great impact on the expected relaxation times. In some experiments as reported in the literature lipids were dissolved in ethanol before injection into the buffer solution. Possible incorporation of ethanol means an impurity and therewith an influence on the transition profile. Faster relaxation processes would be expected. All in all it is difficult to compare the different results with ours, because of the lack of heat capacities or transition profiles in general. We cannot rule out the existence of faster relaxation processes, but it is surprising that non of the cited papers with exception of Grabitz et al. (2002) report about relaxation times for suspensions of multilamellar vesicles up to $30 \mathrm{~s}$. One reason might be problems in the accuracy of temperatures, but also small changes in the experimental system and impurities. We applied a thermodynamical evaluation, where other experiments were analyzed by optical means. With out measurements we evaluated macroscopic relaxation of domains formation, where other methods measure microscopic processes.

Another possibility for the study of relaxation processes is applying ultrasonic methods. Three relaxation processes were found (Halstenberg et al., 2003). Two are seen as being associated with rotational isomerization of hydrocarbon chains and diffusion in the $n s$-regime and a last one with critical fluctuations in chain state in the $10 \mu s$ time regime. Again, no slower processes are found. This might be since we monitor macroscopic processes, whereas ultrasonics measures microscopically ones.

Ac-calorimetry has been used to study relaxation phenomena too (Yao et al., 1994; Nagano et al., 1995). An oscillating heat input results in oscillations in temperature. The amplitude of these oscillations depends on heat capacity. One can adjust different frequencies. From the temperature oscillations one can calculate a heat capacity. If relaxation processes are slower than the oscillations the sample cannot get into its equilibrium and heat capacity values depend on frequency. This allows one to estimate maximal relaxation times. With this technique it has been found that maximal relaxation times are $120 \mathrm{~s}$ (MLV DMPC), $260 \mathrm{~s}$ (MLV DPPC) (Yao et al., 1994) and $<3 s$ (LUV DMPC). In the case of the MLV systems relaxation times are higher then ours. Results on LUV DMPC membranes agree with ours. In principal one can determine relaxation times in dependence on temperature. Practically, this is, however, hard to realize. For each temperature one needs to determine the frequency when the heat capacity does not depend on the frequency any more.

The group of Biltonen used a volume perturbation calorimeter to analyze relaxation processes (Johnson et al., 1983). On MLV lipid vesicles they report relaxation times up to $4 \mathrm{~s}$ (van Osdol et al., 1991). They describe that one finds a pronounced maximum slightly above the melting temperature. Relaxation times on LUVs are about $80 \mathrm{~ms}$ over most of the transition range. The anesthetic dibucaine results in faster relaxation process by a factor of two (van Osdol et al., 1992). The last finding agrees qualitatively with our expectancy. In general relaxation times are again faster than ours. Possibly this is due to the experimental setup of the volume perturbation calorimeter. The pressure in this method oscillates by a value which corresponds to a shift in temperature of $0.1 \mathrm{~K}$. This corresponds to the transition half width of MLVs. The volume perturbation calorimeter does not allow a well defined state. The response might be dominated by fast relaxation processes. Still, in this context it seems puzzling, that relaxation times of LUVs do not show any temperature dependence. A shift in temperature of $0.1 \mathrm{~K}$ should not have influence on the relaxation behavior, since transition half widths of LUVs are bigger.

So far our measurements on one component lipid systems have been compared to results of other groups on similar model membranes with one lipid species. Relaxation times range from the $n s$ to the min time regime. The discrepancy might occur because of experimental 
limitations or because different methods monitor other relaxation processes. Most studies agree on a slowing down of relaxation processes in the transition regime.

\section{Membranes of Binary Lipid Mixtures}

Biltonen and Ye (1993) applied the use of volume perturbation calorimetry to binary lipid mixtures. They describe that relaxation phenomena consist of more than one decay. Relaxation times are smaller than the ones of unitary lipid samples. In equimolar mixtures of different kinds of lipids they asserted that in each case two relaxation time maxima were found. This was even when just one maxima in heat capacity was measured.

We have tried to extend our studies to two component lipid systems. Systematic experiments on DMPC:DSPC 90 : 10 mixtures failed. A collection of relaxation times on just a few temperatures was successful. Problems are probably due to sinking of the heavy MLV vesicles in the pressure cell. The few successful experiments, however, suggest that temperature curves can be described by one relaxation process. Relaxation times lie in a regime as it would be expected from heat capacity and the averaged phenomenological constant of MLV vesicles. A linear relation between relaxation times and heat capacity seems to be the case, as well. Still, this statement has to be taken with great care because of the limited data.

Jørgensen et al. (2000) and de Almeida et al. (2002) report about relaxation processes in two component lipid mixtures on the order of hours. It is claimed that non-equilibrium processes lead to the formation of local lipid structures on various length and time scales (Jørgensen et al., 2000). Using fluorescence techniques and infrared spectroscopy they describe relaxation times up to hours. With our method we gained relaxation processes for DMPC:DSPC 90 : 10 (MLV) up to several seconds. Biltonen and Ye (1993) write that their long relaxation times were around a second, which they relate to diffusion.

Our results from autocorrelations of enthalpy traces and from temperature jump simulations can conclude that there is a fast and a slow relaxation process. The first one is accompanied by a big change in enthalpy and the second one with smaller enthalpy changes. The fast relaxation time is due to changes in lipid chain state. The second process is dominated by diffusion. These conclusions agree with the findings and the conclusions of Jørgensen and Mouritsen (1995). They performed Monte Carlo simulations on a two component lipid membrane described by a ten state model (Pink et al., 1980). Domain formation processes were followed after a sudden change in temperature. Jørgensen et al. (2000) and de Almeida et al. (2002) chose to focus on the slow relaxation process, whereas our experiments are sensitive to enthalpy changes and therewith the fast process dominates the signal. This might be an explanation of the differences in the time scales. We can rule out that our measurements, even though not systematically successful, show long relaxation processes up to hours. If further successful experiments on two component mixtures can be performed they can deepen the understanding of relaxation processes in lipid mixtures and in biological membranes in general.

\section{Summary and Conclusion}

Our study has confirmed that time scales of domain formation processes are altered by small molecules like anesthetics or neurotransmitters and antibiotics. In all cases relaxation times are proportional to heat capacity. Relaxation behavior of domain formation in binary lipid 
mixtures becomes more complicated, but still relaxation times are related to heat capacity, even though fast and slow processes might occur. Domain formation can occur on different time scales.

\subsubsection{Domain Formation and Fluctuations}

Heterogeneities in biological membranes play a role in biological function (Chapman, 1971; Träuble, 1971; Sackmann, 1984; Brown and London, 1998). One putative reason for such heterogeneities are the formation of lipid domains. As seen (see sec. 4.5) these ones can be on the order of system size (macroscopic) or independent of system size (microscopic). The size distribution of clusters was extensively studied by Sugar et al. (2001) using a similar model like ours. In the latter study, however, the authors purely focused on geometrical properties, but neglected fluctuation properties of the system. System and domain size and accompanying fluctuations in chain state have been the emphasis of our study. We claim that for a complete physical understanding fluctuations need to be considered.

We report about domain formation processes on different length scales (see figs. 4.30 and 4.31), as it is also verified experimentally (Korlach et al., 1999; Bagatolli and Gratton, 1999; Nielsen et al., 2000b; Kaasgaard et al., 2002; Tokumasu et al., 2003). We report about macroscopic and microscopic phase separation. In the case of macroscopic domain formation we find microscopic domains in macroscopic ones. These ones can be of different chain order or different species. The first case is especially interesting since these domains display large local fluctuations. In general, fluctuations are enhanced close to domain interfaces. This is also true for interfaces between lipids and peptides (Ivanova et al., 2003). Addition of small molecules like alcohols (see fig. 4.30) or compositions of other kinds of lipids (Bagatolli and Gratton, 2000) can alter domain formation. Formally speaking, a decrease in order-disorder interaction parameters leads to microscopic phase separation. In these cases fluctuations dominate the matrix. Fluctuations have been experimentally verified by neutron scattering (Czeslik et al., 1997; Winter et al., 1999) or AFM (Nielsen et al., 2000a). They become evident through a power law behavior of the structure factor. In principal we could evaluate our snapshots of the lateral lipid chain state distribution by means of a structure factor or pair-pair correlations (Jørgensen and Mouritsen, 1995). Even though this has not been done it is evident that our results support a suggestion of Sackmann (1984): "Consequently the lipid heterogeneity is rather due to extended concentration fluctuations which form and dissolve rapidly.". This is true for small domains. Domains with high cholesterol and sphingolipid concentration ("rafts") are assumed to be in the $n m$-scale (Simons and Vaz, 2004). These should be dynamic and not static like the term "raft" suggests. Fluctuations enter the physical picture. "Rafts" are discussed to play an important role in trafficking processes (Brown and London, 1998), therefore a detailed understanding of the underlying physical processes might be necessary. Our study adds to this understanding.

\subsubsection{Summary}

Diffusion, relaxation, domain formation and fluctuation properties change when artificial membranes undergo a melting transition. The details depend on the used lipid species, the type of lipid vesicle or the addition of non-lipid molecules.

In the melting transition regime fluctuations are enhanced, domains on different length 
scales might form, relaxation processes become slow and the diffusion properties deviate from a normal diffusion behavior.

One cannot regard these processes independently. It is a direct consequence of the melting transition of artificial membranes.

\subsection{Biological Relevance}

In this work experimental and simulation methods are combined to deepen the physical understanding of melting transitions in artificial lipid membranes. The focus was put on diffusion $^{4}$, relaxation ${ }^{5}$, domain formation and fluctuation properties of the chosen model systems in the transition regime.

The physics of the presented findings is interesting in itself. Further, a major question is whether biochemical reactions in cells are purely based on biochemistry or whether melting phenomena in biological membranes might be involved in the triggering of physiological function. Answering this question, however, is not trivial. The complexity of biological membranes makes it difficult to unambiguously assign a physiological process to a physical mechanism. In the following sections we discuss about a possible role of melting processes and their implications on domain formation, fluctuation, diffusion and relaxation processes in cell physiology.

\subsubsection{Phase Transitions, Lipid Domain Formation and Protein Activity}

Already in the early 1970s the idea that phase transitions of lipid membranes and thereby membrane heterogeneities display an important role was formulated (Chapman, 1971; Träuble, 1971; Sackmann, 1984). A very prominent example of an enzyme with an activity depending on inhomogeneities of the lipid membrane is phospholipase $A_{2}\left(P L A_{2}\right)$. It hydrolyzes phospholipids into fatty acids and other lipophilic substances. Phospholipases in general play an important role in the structure and function of membrane systems by controlling the level of free fatty acids (van den Bosh, 1980). First reports on the activity dependence on the phase state of model systems dates back to the years 1974 and 1975 (Kamp et al., 1974, 1975). Already then they concluded that a hydrolysis is only possible at the transition temperature of the liposomes used. These findings were supported by Gabriel et al. (1987) and especially the group of R. Biltonen (Lichtenberg et al., 1986; Burack and Biltonen, 1994; Hønger et al., 1996, 1997). A work using fluorescence microscopy could verify that $P L A_{2}$ starts to hydrolyze lipids in the ordered state starting from the domain boundaries (Grainger et al., 1989). A second example is protein kinase $\mathrm{C}$ (PKC) which modifies proteins by chemically adding phosphate groups to them. This enzyme reflects importance in the regulation of various physiological responses like cell growth and differentiation (Nishizuka, 1989; Kikkawa et al., 1989). In this case, it was shown that the lateral structure of the lipid bilayer controls the activation of the enzyme (Bolen and Sando, 1992; Dibble et al., 1996). In the case of PLA Biltonen (1990) relates the rate of activation to thermodynamical fluctuations: "It thus appears that the rate of activation of the enzyme requires that the substrate exists in a phase-transition region and that the rate of activation is proportional to the magnitude of the thermodynamic fluctuations characteristic of the system.". Exactly in the phase transition regime fluctua-

\footnotetext{
${ }^{4}$ Experimental measurements were performed by A. Hac (Hac, 2004).

${ }^{5}$ Experiments on DMPC/Gramicidin A systems were conducted by M. Gudmundsson (Gudmundsson, 2004).
} 
tions happen on longer time scales. This can be modulated by processes which change the phase behavior. In experiments, this can be a change in temperature, which, however, is not likely in nature. Variations in $\mathrm{pH}$ or ion concentration (Träuble and Eibl, 1974) or addition of small molecules like anesthetics and neurotransmitters can influence the domain structure and fluctuation properties. The activity of the enzyme can be regulated in this way. These considerations might play a general role since the activity of proteins depends on the lipid composition and on lipid state (Lee, 2003, 2004).

Altering protein diffusion in biological membranes by changes in the lateral structure is another possible mechanism controlling biochemical reactions. Reaction yields between two reactants depend on the lateral heterogeneity and provide an on- and off-switch (Melo et al., 1992; Vaz and Almeida, 1993; Thompson et al., 1995; Hinderliter et al., 2004; Salinas et al., 2005). Again, addition of small molecules or using different lipid species alters the life times of domains and the details of the lateral structuring. Perturbations of the lipid membrane might induce an increase or decrease of protein activity because of a change of the lateral structuring with its characteristic time scales.

Whether lipid domain formation is important in the function of cells remains an open question. It is evident that they provide a possible role, but in biological membranes different kinds of domains can be found (Edidin, 1992; Jacobson et al., 1995; Almeida and Vaz, 1995; Edidin, 1996; Saxton, 1999). Lipid domains are one possible hindrance to diffusion, but also protein aggregation, or the cytoskeleton ${ }^{6}$. The lateral structure of biomembranes is not just governed by lipid-lipid, but also lipid-protein and protein-protein interactions. The formation of inhomogeneities depends on the interplay between them.

\subsubsection{Passive Permeation}

Cell membranes provide a barrier to ionic solutes like protons, calcium, potassium and sodium. This is necessary to maintain ion gradients, which are believed to be necessary for bioenergetics function and nerve activity (Paula and Deamer, 1999). Biological membranes are semipermeable meaning that they are a lower barrier towards the flux of water, oxygen or carbon dioxide than to the flux of ions such as protons and sodium. One divides between passive and active permeation processes. Ion pumps requiring energy stand for the latter class. Textbooks teach that passive permeation from the side of high concentration to low concentration can be achieved through a transport through the membrane itself, peptide induced pores, channel proteins or using conformational changes of carrier proteins. Nowadays, it is believed that mainly peptide induced pores or opening or closing events of channel proteins are responsible for the regulation of membrane permeability.

In a recent study Makarov (2005) relates structural fluctuations to the rate of pore formation in lipid membranes. In the cited work, the permeability of the fluorescence dye rhodamine through a lipid vesicle is measured. Results are compared to a numerical model. It is shown that the permeability of a pure lipid membrane increases in the phase transition regime. In their model pore formation processes are coupled to area fluctuations. Adding the pore forming peptide gramicidin A leads to a decrease of permeability at the temperature corresponding to the transition midpoint temperature of the lipid vesicle without peptide. This is attributed to decreasing local fluctuations as it can be deduced from the heat capacity profiles of the

${ }^{6}$ The cytoskeleton is a dynamic structure which is believed to maintain cell structure, enable cell motion and directed intra-cellular transport. 
lipid/peptide mixtures. Fluctuations are high at domain boundaries and at lipid/peptide interfaces (Ivanova et al., 2003). Makarov (2005) shows that pore formation mainly appears at lipid domain and lipid/peptide interfaces. The addition of gramicidin A also shifts the regime of enhanced permeability related to its influence on the melting behavior. The maximum permeability, however, is less than for the lipid vesicle without peptide.

These findings allow to suggest that physical properties of lipid membranes might be involved in the regulation of membrane processes which require permeation. Addition of molecules like anesthetics might perturb the lipid membrane instead of directly influencing the activity of channel proteins or the occurrence of peptide induced pores. This also allows to raise the question whether channel proteins and pore forming peptides might act through perturbing the membrane and inducing strong local fluctuations in their vicinity. This of course means that the role of channel proteins and pore forming peptides is another one than the traditional picture proposes. A combination of mechanisms might be possible too.

Pore forming peptides can induce large pores (Oliynyk, 2005). Therefore, it would be surprising if they purely act through changing the physical properties of the lipid membrane. In the case of channel proteins it is different. This topic is discussed in the next section.

\subsubsection{Nerve Pulse Propagation, Ion Channels and Anesthesia}

Nerve pulses are seen as a propagation of an action potential ${ }^{7}$ along a nerve. The accepted theory is the one by Hodgkin and Huxley (1952). It represents the membrane by a Kirchhoff circuit. The membrane displays a constant capacitance and shows a conductivity to potassium, sodium and a leakage current. It is believed that opening and closing events of specific protein channels control the conductivity of potassium and sodium during an action potential.

\section{Ion Channels}

Static measurements on systems of the potassium and sodium conductivities are functions of time and changes are interpreted as resulting from opening and closing of specialized channel proteins. They are interpreted as specific "ion channels". Single channel current recordings were first presented in 1976 (Neher and Sakmann, 1976), where macroscopic conductance fluctuations had been reported before (Bean, 1973). An example of single channel recordings can be seen in the left panel of fig. 5.1. Current fluctuations of a membrane with channel proteins show characteristic steps which are taken as evidence of the opening and closing of protein channels. Inducing pore forming peptides into lipid membranes also results in discrete conductance steps (Hladky and Haydon, 1970; Ermishkin et al., 1976; Kappel et al., 2000). However, also lipid membrane systems without any protein or peptide show the characteristic behavior (Antonov et al., 1980; Boheim et al., 1980; Kaufmann et al., 1989; Woodbury, 1989; Antonov et al., 2005).

(Antonov et al., 1980; Boheim et al., 1980; Antonov et al., 2005) relate their measurements to lipid membrane phase transitions. In the right panel of fig. 5.1 a graph taken from Antonov et al. (2005) is displayed. A DPPC lipid membrane was taken and the sample temperature was varied. The upper and lower traces were measured at temperatures well above and well below the main phase transition temperature. The remaining trace was recorded at the main phase transition temperature. The trace shows steps in the electrical current,

${ }^{7}$ An action potential is a wave of electrical discharge traveling along membranes. 

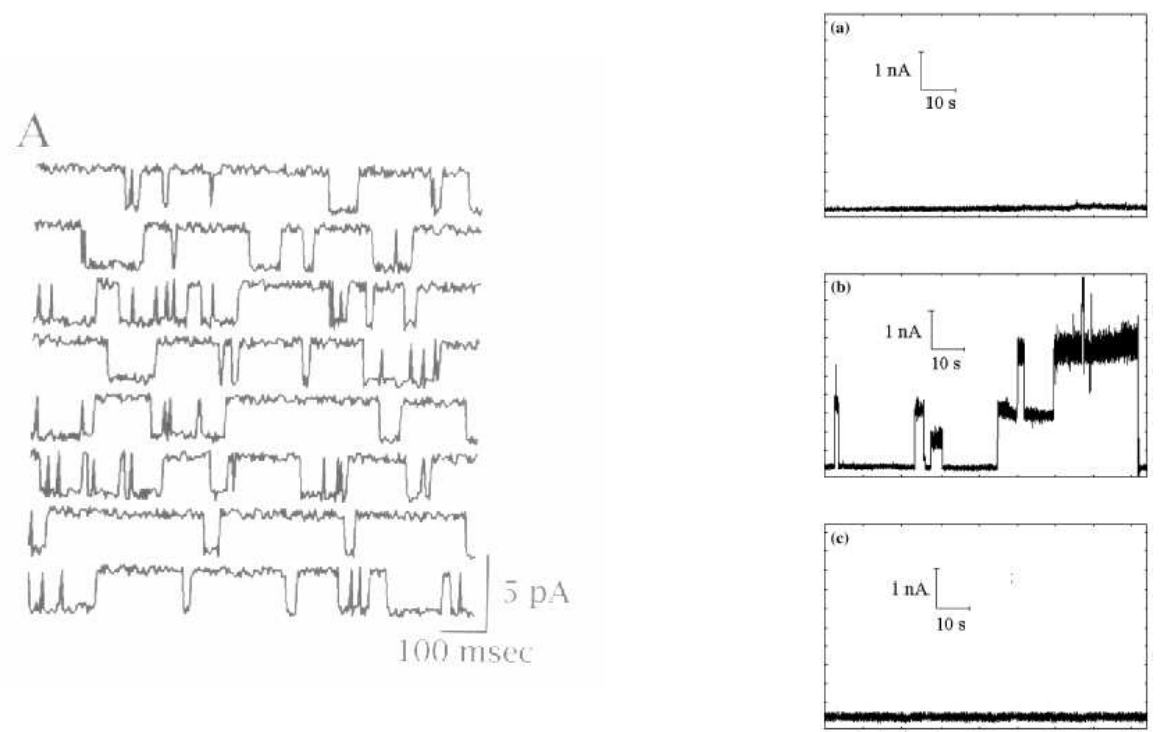

Figure 5.1: Electric current fluctuations. (left) Current traces are collected from measurements on a membrane system with channel proteins. Steps in the traces are interpreted as being opening and closing events of single channels. The picture is fig. 8.7 of Johnston and Wu (1997). (right) Current traces are monitored on a DPPC membrane at different temperatures. Below and above the main phase transition temperature one finds only noise, where in the main phase transition regime current fluctuations appear. These one show the same characteristic steps as they are monitored with the presence of channel proteins. The presented traces are taken from Antonov et al. (2005).

whereas the traces monitored outside of the transition regime only show noise. One should note the different time and current scales in the two panels of fig. 5.1. These findings challenge the traditional interpretation of single channel events. Previously the term "ion channels" was used for pore formation in pure lipid membranes (Kaufmann et al. (1989)).

It is well known that permeability increases during an action potential (Keynes, 1951). As discussed in the previous section passive permeation through the lipid membrane is related to thermal fluctuations (Cruzeiro-Hansson and Mouritsen, 1988; Corvera et al., 1992; Makarov, 2005). The rate of pore formation increases in the phase transition regime, as does the life time of these pores (Makarov, 2005). This behavior agrees with our results, that relaxation times and the time scale of fluctuations display a maximum in the transition midpoint. Depending on the addition of small molecules or using unilamellar instead of multilamellar vesicles one finds differences in the absolute times. One might assume that both time scales are related. If the life time of pores and the time scale of thermal fluctuations of the lipid membrane are the same one would expect that traces of current fluctuations show signals in the time range of the relaxation times. For a planar DPPC bilayer one would expect signals in the second regime. Antonov et al. (2005) using a planar DPPC system found traces with signals up to $10 \mathrm{~s}$, but also shorter ones (around $1 \mathrm{~s}$ ). One problem of the measurements lies in the inaccuracy of the temperature $( \pm 0.5 \mathrm{~K})$ and the usage of $n$-decane/chloroform/methanol solution during the preparation procedure which was not completely removed. 25 years earlier the same group reported about signal durations of about $1 s$ using planar DSPC bilayers (Antonov et al., 1980). DSPC membranes display a more cooperative melting than DPPC membranes. Following our considerations longer duration times would have been expected. 
However, due to the experimental preparation, inaccuracies in the temperature control and problems with signal recording one cannot rule out that time scales are shorter than expected due to the experimental conditions. Measurements on lipid mixtures show a reduction in signal duration (Kaufmann et al., 1989). This would be expected from our considerations. Lipid mixtures show a broad transition regime. Absolute heat capacity values are decreased in comparison to the one component systems. This means that relaxation times are shorter. In Kaufmann et al. (1989) experiments were performed at room temperature. Heat capacity values are not known, as is not the melting behavior of the lipid membranes used. Still, the findings agree qualitatively with our results and considerations. Proteins or peptides lead to a broadening of heat capacity profiles. Maximal relaxation times therefore decrease. Current fluctuations on membrane systems with proteins or pore forming peptides show signals with shorter duration as it is seen in fig. 5.1 (left panel), as well. Our considerations basically mean that relaxation processes, as measured in the context of this thesis, determine the time scales of ion conductance measurements.

The traditional picture of conductance through protein channels and peptide pores and conductance through pure lipid membranes is, however, challenged by experiments on silicon rubber or synthetic membrane filters (Neher et al., 1978; Sachs and Qin, 1993; Lev et al., 1993). Erwin Neher and Bert Sakmann received the Nobel prize in medicine for their recordings on "single ion channels" in 1991. Even these two authors reported about "behavior contrary" to what was expected performing control experiments on silicon-rubber (Neher et al., 1978). These measurements showed "ion channel" behavior too. This was dismissed explaining it with technical difficulties. Sachs and Qin (1993) report the same behavior and conclude that one has to be careful with the interpretation of experiments on "unfamiliar preparations". Experiments on synthetic membrane filters also show the same characteristic current fluctuations (Lev et al., 1993). In all cases, as well the one on pure membrane, membrane/protein and membrane/peptide systems, one finds ion selectivity. This means that different ions permeate differently. It seems that for the existence of characteristic current fluctuations some kind of pore is necessary. In the measurements of Lev et al. (1993) the same amount of pores with the same size is present at all times. This raises the question whether one can interpret traces by means of opening and closing times of pores and channels. A definitive answer cannot be stated at the moment. Still, especially the work by Antonov et al. (2005) verifies that the phase transition and the enhancement of fluctuations might play a major role. They showed that at temperatures (below and above the phase transition temperature), where there is no pore formation, one only measures noise. In the phase transition regime current fluctuations occur.

Pore forming peptides and channel proteins are present in biological membranes. Thus, one needs to answer the question about the role of "channel-proteins" and "pore forming peptides". One interpretation which has already been raised above is that fluctuations at lipid/protein and lipid/peptide interfaces are increased and pore formation in their vicinity is enhanced. It has already been shown that spontaneous opening of "sodium channels" depends on lipid composition and temperature (Duch and Levinson, 1987). The traditional picture of a biological membrane is that it is in a "ld" phase. If this is the case the pure lipid membrane would not show the characteristic current fluctuations and the task of "channel-proteins" and "pore forming peptides" might be to ensure the control of membrane permeability. The latter interpretation corresponds to the accepted picture. Using "ld" membranes and pore forming peptides one finds characteristic current fluctuations (Hladky and Haydon, 1970; Ermishkin et al., 1976; Kappel et al., 2000). It might be that the truth lies in between both 
views.

\section{Nerve Pulse Propagation}

Permeability increases during an action potential (Keynes, 1951), which is seen as occurring because of opening of protein channels. If considering the passive permeability of a lipid membrane an increase of it means that thermal fluctuations have enhanced. This can be achieved through going from a phase state away from the transition regime into it. If this might happen in a nerve membrane during an action potential further physical parameters should change. It was monitored that temperature increases adiabatically during an action potential (Tasaki and Byrne, 1993) and structural changes of the membrane are present (Tasaki et al., 1968; Tasaki and Byrne, 1992). These processes cannot be explained by the model of Hodgkin and Huxley. Keeping, however, the interpretation of opening and closing events of protein channels the favored explanation is that these processes arise from conformational changes of macromolecules (Kobatake et al., 1971; Tasaki and Byrne, 1992). Another view is that a phase transition of the cytoskeleton induces an action potential and structural changes (Pollack, 2001). This latter view contradicts the concept of Hodgkin and Huxley.

In a recent publication Heimburg and Jackson (2005) present a theory which treats the nerve pulse as a soliton which naturally arises after a jump from the "ld" phase through or into the phase transition regime. From our point of view the case of a jump into the phase transition regime would be interesting. As seen in our experiments (see fig. 2.6) a jump from the "ld" phase into the transition regime results in an increase of temperature. Diffusion gets slower as it is measured during a nerve pulse (Kobatake et al., 1971). Fluctuations increase and thereby membrane permeability. If the nerve pulse is a soliton induced through a change in the phase state of a nerve membrane, the importance of domain formation on characteristic time scales is obvious. However, the question how a biological system might induce a change of the phase behavior arises. One possibility was described by Kaufmann and collaborators (Kaufmann and Silman, 1980, 1983b,a). The idea is based on the fact that in the presence of acetylcholine (ACh) acetylcholinesterase (AChase) induces ion channel properties (Kaufmann and Silman, 1980). The hydrolysis of ACh through AChase is important during synaptic transmission. The monitoring of ion channel behavior is believed to arise from a local decrease in $\mathrm{pH}$ which leads to a protonation of lipid head groups. This again results in an increase of the transition temperature ${ }^{8}$ and induces a change in phase behavior (Kaufmann and Silman, 1983b,a). In general each process leading to a change in $\mathrm{pH}$ or ion concentration leads to an altering of the phase behavior. Our findings are especially interesting in the context of the theory of Heimburg and Jackson (2005). All in all a deeper understanding of melting transitions and accompanying domain formation, fluctuation, diffusion and relaxation properties can lead to new insights concerning permeability and nerve pulse propagation. Despite the current believe still many open questions need to be answered. Some solutions might provide new ways of thinking.

\section{Anesthesia}

Heimburg and Jackson (2006) try to apply their treatment of a nerve pulse as a soliton on the action of general anesthetics. As it is shown in this thesis (see sec. 4.3) and in other

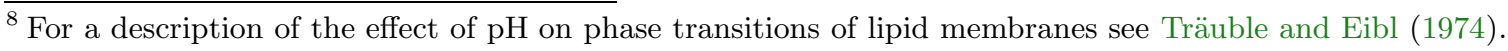


works (Lee, 1976a; Tamura et al., 1991; Mengel and Christiansen, 2005) general anesthetics lower the transition temperature of lipid membranes. If a nerve pulse arises after a jump into a transition regime a reduction in melting temperature would result in a higher threshold (Heimburg and Jackson, 2006). This might induce the anesthetics effect of general anesthetics. In favor of a treatment like this is that the anesthetics effect of general anesthetics is additive and unspecific, suggesting an underlying physical effect (Overton, 1901). Higher pressures result in higher melting temperatures. Following the arguments of Heimburg and Jackson (2006) this explains the reversal of anesthetics effects of general anesthetics at high pressures (Tamura et al., 1991) since the threshold of the emergence of a soliton decreases again. That anesthetics act through perturbing the lipid membrane was already suggested even before the postulation of the fluid mosaic model (Overton, 1901). In the recent years this has been dismissed because of several reasons. One was a cut-off of the anesthetics action of alcohols after a certain chain length. It could, however, been shown that alcohols not showing anesthetics action shift the melting temperature to higher temperatures (Tamura et al., 1991). This again favors a theory which treats the lipid membrane phase transition as an important aspect of nerve pulse propagation. The stereoselectivity of some anesthetics was taken as another evidence against a mechanism based on the lipid membrane (Tomlin et al., 1998). Two isomers of entomidate disrupt the lipid bilayer in the same way, but display different anesthetics potencies. This seems to be the biggest challenge towards a theory based on a melting temperature reduction of anesthetics. However, it cannot be excluded that in biological membranes binding effects towards proteins change the disturbing effect. Especially, it has been suggested that anesthesia should be seen as a combination of effects on lipids and proteins (Ueda and Yoshida, 1999).

It seems worthwhile to follow ideas relating influences on the melting behavior of lipid membranes to problems like nerve pulse propagation and "ion channel" conductances.

\subsubsection{Speculations}

In the previous sections possible roles of the investigated processes in biological function were discussed. In some cases these considerations challenge traditional pictures. They are based on a biological membrane in which heterogeneities induced through lipid domains formation play an essential role.

The fluid mosaic model by Singer and Nicolson (1972) has been challenged the recent years. It is obvious that inhomogeneities are present (Edidin, 1992; Jacobson et al., 1995). This heterogenous lateral structure, however, has several reasons, where lipid domains might be one. Interactions in biological membranes are complex and changing outer parameters like ion concentration or $\mathrm{pH}$ might have a series of consequences which again influence each other. Focusing the view onto influences of parameters like $\mathrm{pH}$ or ion concentration on lipid membrane melting properties, one easily understands that changes in $\mathrm{pH}$ or $\mathrm{Ca}^{2+}$ concentration might have considerable consequences on the structuring induced through changes of the lipid membrane lateral order. These thoughts are especially interesting since a biological system is a system which is not in equilibrium. Local changes in $\mathrm{pH}$ or ion concentration could induce local changes in membrane structure and thereby a local control of biological function. This is a picture of a biological membrane which is highly dynamic, not in equilibrium and physical properties trigger indirectly physiological function. 


\subsection{Future Research Perspectives}

In this thesis studies on diffusion, relaxation, domain formation and fluctuation properties of lipid membranes are presented. Further, it is suggested that these processes might play an important role in the physiology of biological cells. To gain direct evidence is complicated because of the complexity of these systems. It is crucial to perform experiments, which can indeed allow an analysis by physical and especially thermodynamical means. In general, it cannot be excluded that nature has found ways to use different strategies to accomplish biological function. Evidence on one system against a certain mechanism does not automatically rule out the existence of this mechanism in another system. The interplay of different strategies in one system might be a possibility.

From the results presented in this work one can already deduce ideas for further experiments. In the last sections statements were made which need experimental verification. Next, general suggestions about the direction in which future research should go in order to evaluate, physical effects as triggers of biological function are given too.

\subsubsection{Further Experiments}

In the following some ideas about possible extensions of the presented experiments and results are given. In detail, these are suggestions about diffusion, relaxation and fluctuation studies.

\section{Diffusion}

In this thesis shown is that the numerical model used can predict measured FCS autocorrelation profiles. Fluctuations in chain state influence diffusion properties. Fluctuations and domain structure play an essential role. Effects of anesthetics or neurotransmitters on diffusion properties are suggested through simulation studies with changed ordered-disordered interaction parameters. These findings could be easily verified performing diffusion experiments on lipid membranes with addition of the mentioned molecules. A problem of course is that anesthetics and neurotransmitters do not only reduce interaction parameters, but shift the transition profile to lower temperatures. A careful analysis should allow to test the predictions of this thesis.

Another experiment concerning diffusion processes is the diffusion of proteins. This can be done on a numerical level, but also experimentally. The numerical method requires the introduction of interactions between lipid chains of different state and species with the protein and an interaction between proteins themselves. A diffusion rate of protein to lipid and protein diffusion in different lipid membrane domains also needs to be formulated. In dependence on temperature protein diffusion can be studied. These studies can mimic diffusion after a distortion of the domain structure through addition of anesthetics or neurotransmitters, too.

\section{Relaxation Measurements}

For various one component lipid membrane systems with or without addition of small molecules it was shown that relaxation times are proportional to heat capacity. Simulations on binary lipid systems suggest that in the transition regime the slow and fast relaxation are present and they are related to heat capacity. One finds a fast and a slow relaxation 
process. After a pressure perturbation the fast process is accompanied by high changes in enthalpy, the slower ones with smaller changes in enthalpy. Therefore, it is expected that using our method monitoring the temperature course after a pressure change is dominated by the faster process. This one is the one which can be monitored. These experiments would allow to investigate domain formation processes in two component mixtures. An important step towards biological membranes in which several lipid species are found. Of course an extension of the relaxation studies towards biomembranes is wishful as well. In this case using a chip calorimeter (see ch. 2.5) with a presumable higher time resolution might turn out to be helpful. The design of the chip calorimeter should also allow to study relaxation processes induced through perturbations by changing $\mathrm{pH}$ or ion concentration. Measurements combing perturbation techniques and the monitoring of protein activity seem to be challenging, but might be helpful to understand whether relaxation processes after local $\mathrm{pH}$ or ion concentration changes might be a possible trigger mechanism in biological systems.

\section{Thermal Fluctuations}

Local fluctuations are enhanced close to domain interfaces. Makarov (2005) relates pore formation and therefore passive permeation to fluctuations. Pore formation is enhanced in the case of strong fluctuations. In the case of macroscopical phase separation one would expect pore formation along domain boundaries. It might be interesting to combine optical detection of domain structures as it is done with confocal fluorescence microscopy and ion current measurements. It is expected that in areas where there are no domain interfaces one does not monitor characteristic steps in current traces, but at domain boundaries they should appear. A problem concerning these measurements might be of course that confocal fluorescence microscopy does not show possible micro-heterogeneities. They might also result in a higher probability of pore formation. This problem should, however, be possible to minimize using two phospholipid species with big differences in chain length. It is expected that traces show different characteristics, depending on the measurement position.

It is also wishful to combine measurements of current fluctuations on different one component lipid systems at different temperatures. It is interesting whether timescales as they can be found in current traces differ from system to system and show a temperature dependence, which can be related to the melting behavior. This is expected following the arguments concerning fluctuations, their time scales as gained from pressure perturbation experiments and pore formation processes.

A few experiments are suggested. They directly follow from the presented studies of this thesis. In the following paragraph a more general view of future research is presented.

\subsubsection{General Suggestions}

As mentioned biological membranes are very complex. Not that there are just different kinds of lipid species, but also proteins, peptides, enzymes, sugars, the cytoskeleton and so on. Lipids have an influence on protein activity, lipid domains play an important role in trafficking processes. Lipid metabolism is important for a proper function of cells too. A crucial question is whether physiological function in biomembranes is purely based on biochemistry or whether physical effects of the lipid membrane may provide a control mechanism. That this may be the case is argued in previous sections. 
Future research should try to focus on a clear thermodynamical description of these systems. One has to understand how $\mathrm{pH}$ changes, ion concentration changes might or might not trigger biochemical reactions through changing the phase behavior of the lipid membrane. Various molecules which are related to physiological function could also act through influencing the melting properties of a biological membrane. Diffusion pathways might become influenced and therewith probabilities of reaction cascades. Controlling on- and off states of proteins could be possible changing the lateral membrane structure too.

Biological membranes relax into equilibrium if outer parameters like $\mathrm{pH}$ or ion concentration are constant. A biological system, however, is not in equilibrium if alive. Changes in $\mathrm{pH}$ or ion concentration, $\mathrm{pH}$ or ion gradients can have major effects on the physical behavior of a biological membrane. Biochemical studies, in which outer parameters are changed might in the control of physiological function suggest biochemical mechanisms. This interpretation might be possible even if the underlying mechanism is rather of physical nature. Whether there is a dependence on temperature might be helpful in learning more about underlying processes. Since living systems are not in equilibrium studies applying non-equilibrium methods gain importance. This kind of research has not gained much attention in the past. 


\section{Appendix A}

\section{Abbreviations}

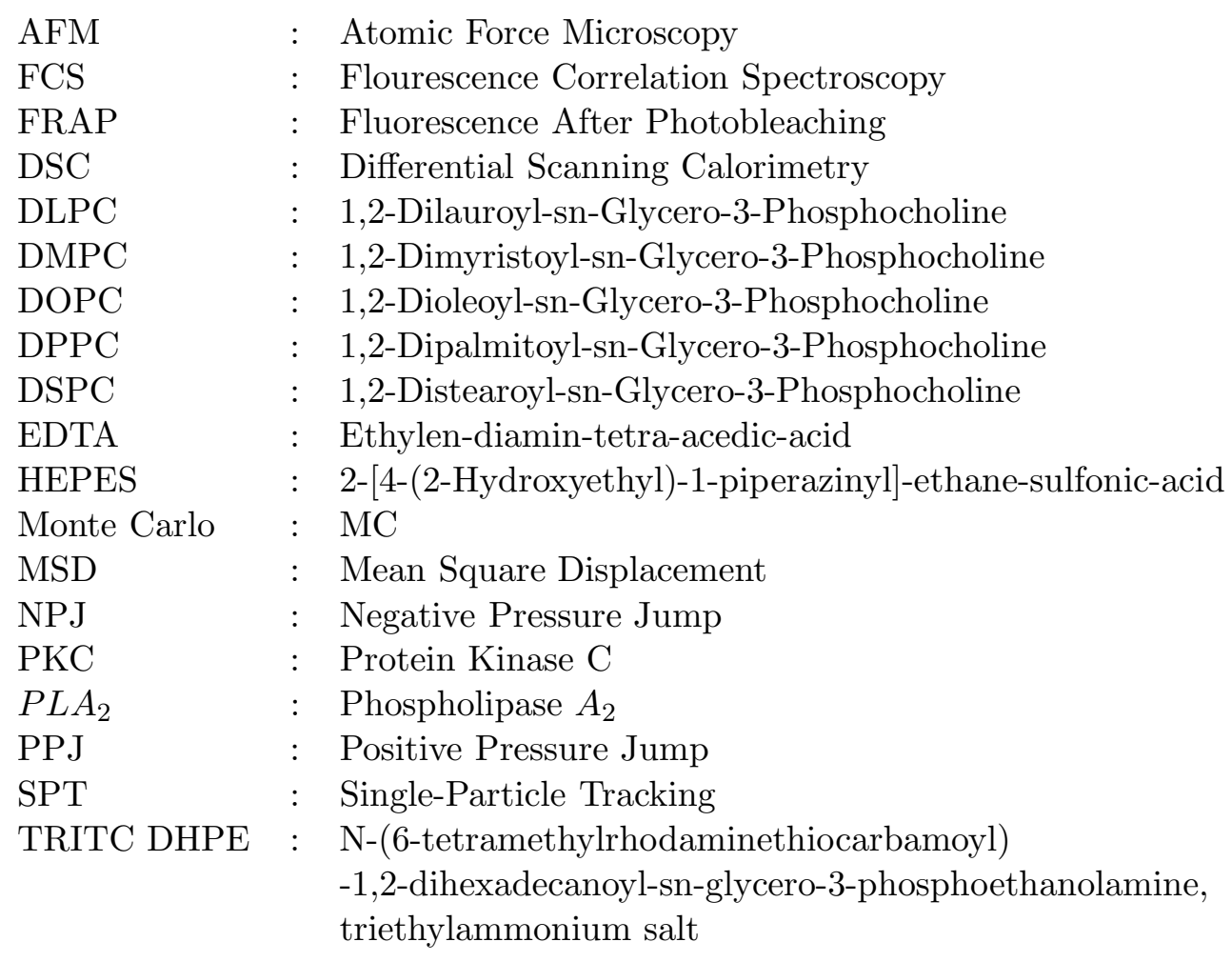




\section{List of Figures}

1.1 Animal Cell . . . . . . . . . . . . . . . . . . . . . 2

1.2 DMPC Structure . . . . . . . . . . . . . . . . . . . 2

1.3 Potential Energy for Rotations of C-C Bonds . . . . . . . . . . . . 3

1.4 Lipid Aggregates . . . . . . . . . . . . . . . . . . . . 4

1.5 Melting Transition of a Lipid Bilayer . . . . . . . . . . . . . . . . . 4

1.6 Structure of Sterols . . . . . . . . . . . . . . . . . . 5

1.7 Phase Diagram and Heat Capacity Profile of a Two Component Lipid System 6

1.8 Confocal Fluorescence Microscopy Picture . . . . . . . . . . . . . . . . 7

1.9 Fluid Mosaic Model . . . . . . . . . . . . . . . . . . . . . . . 8

1.10 Matress Model . . . . . . . . . . . . . . . . . . . . . . . . 9

1.11 Modern Membrane Picture . . . . . . . . . . . . . . . . . . . . . 10

1.12 Gramicidin A . . . . . . . . . . . . . . . . . . . 11

1.13 Serotonin . . . . . . . . . . . . . . . . . . . . 12

1.14 The General Anesthetics Octanol and Halothane . . . . . . . . . . . . . . . 12

2.1 Differential Scanning Calorimetry . . . . . . . . . . . . . . . . . . 20

2.2 Heat Capacity Profiles: Different Pressures . . . . . . . . . . . . . . . . 21

2.3 Pressure Jump Responses: Principle . . . . . . . . . . . . . . . . . . . . . . . 22

2.4 Pressure Jump Responses: Jumps to Different Regions . . . . . . . . . . . . . 23

2.5 Pressure Cell for Method II . . . . . . . . . . . . . . . . . . . 25

2.6 Example Thermocouple Signal after a Positive Pressure Jump . . . . . . . . . 27

2.7 Thermocouple Signal: Original, Perturbation and Lipid Signal _ . . . . . . . 29

2.8 Original and Lipid Signals at Different Temperatures . . . . . . . . . . . . . . 31

2.9 Principle of Fluorescence Correlation Spectroscopy . . . . . . . . . . . . . 35

2.10 Example Fluorescence Intensity Traces and Autocorrelation Curves . . . . . . 36

2.11 NCM-9924 Chip . . . . . . . . . . . . . . . . . . . . . . 38

2.12 Dimensions of the Chip Calorimeter . . . . . . . . . . . . . . . 39

2.13 Chip Calorimeter Perspective Views . . . . . . . . . . . . . . . . 40

3.1 Heat Capacity Profiles: One Component Lipid Systems _ . . . . . . . . . . . 47

3.2 Monte Carlo Steps . . . . . . . . . . . . . . . . . . . . . . 49

3.3 Fluctuations and Excess Heat Capacity . . . . . . . . . . . . . . . . 50

3.4 Autocorrelation of Enthalpy Traces . . . . . . . . . . . . . . . . . . 51

3.5 Fluorescence Trace and Autocorrelation . . . . . . . . . . . . . . . . 52

3.6 Calibration of Exchange Activation Barrier . . . . . . . . . . . . . 53

3.7 Laser Focus in the FCS Simulation . . . . . . . . . . . . . . . . . . . 54 
3.8 Lipid Chain State Distribution and Local Fluctuations . . . . . . . . . . . 56

3.9 Enthalpy Course After a Temperature Jump . . . . . . . . . . . . . . . . 57

3.10 Simulated Enthalpy Traces _. . . . . . . . . . . . . . . . . 60

3.11 Autocorrelations of Enthalpy Traces . . . . . . . . . . . . . . . . 60

3.12 Simulated Heat Capacity Profiles . . . . . . . . . . . . . . . . . . . . 61

3.13 Comparison of Simulated Heat Capacity Profiles and Relaxation Times . . . 61

4.1 Phase Diagram of the DMPC:DSPC Lipid System . . . . . . . . . . . . 64

4.2 Experimental and Simulated FCS Autocorrelation Profiles . . . . . . . . . 66

4.3 Variation of Autocorrelation Profiles in FCS Experiments . . . . . . . . . . . 67

4.4 Autocorrelation Profiles and Dependence on Focus Size . . . . . . . . . . . 68

4.5 Superposition of Diffusion Times . . . . . . . . . . . . . . . . . . 69

4.6 Influence of Different Timescales on Autocorrelation Profiles . . . . . . . . . . 70

4.7 Heat Capacity Profiles With Changed Interaction Parameters . . . . . . . . . 71

4.8 FCS Simulations with Different Interaction Parameters . . . . . . . . . . . . 72

4.9 Heat Capacity Profiles of Pure Lipid Membranes . . . . . . . . . . . . . . . 73

4.10 Influence Of Small Molecules or Peptides On Melting Transitions . . . . . . . 74

4.11 Heat Capacity and Relaxation Times of DMPC (MLV) . . . . . . . . . . . 76

4.12 Temperature Course After Pressure Jump (Method II) . . . . . . . . . . . . 77

4.13 Hysteresis Effects in a DMPC Suspension . . . . . . . . . . . . . . 78

4.14 Transition Enthalpy and Total Area of Calorimeter Response . . . . . . . . . 79

4.15 Relaxation Times: DMPC (MLV) with Method II . . . . . . . . . . . . . 80

4.16 Relaxation Times: DMPC (LUV) with Method II . . . . . . . . . . . . . . . 81

4.17 Relaxation Times: DMPC Membranes (MLV) with 1-Octanol . . . . . . . . . 83

4.18 Relaxation Times: DMPC Membranes (MLV) with Serotonin . . . . . . . . . 84

4.19 Relaxation Times: DMPC Membranes with Gramicidin A . . . . . . . . . . . 86

4.20 Transition Enthalpy and Area of Perturbation and Lipid Signal (Method I) • 87

4.21 Relaxation Times: Large Unilamellar Vesicles . . . . . . . . . . . . . . . . 88

4.22 Relaxation Time vs. Heat Capacity . . . . . . . . . . . . . . . . . . . 89

4.23 Matrix Snapshots after a Temperature Jump . . . . . . . . . . . . . . . . . . 91

4.24 Simulated Temperature Jump Experiments . . . . . . . . . . . . . . . . . . . 92

4.25 Temperature Jump Simulation of a Two Component Lipid Mixture . . . . . 93

4.26 Autocorrelation Curves of Enthalpy Fluctuations . . . . . . . . . . . . . 95

4.27 Enthalpy Correlation: Rate of State Changes and Interaction Parameters . . 96

4.28 Heat Capacity Profiles of an Equimolar DMPC:DSPC Mixture . . . . . . . . 97

4.29 Fraction of Disordered DMPC and DSPC Chains . . . . . . . . . . . . 98

4.30 Fluctuations and Lipid Chain State Distribution . . . . . . . . . . . . . 99

4.31 Snapshots at Different Matrix Sizes . . . . . . . . . . . . . . . . . . . . 101

5.1 Electric Current Fluctuations . . . . . . . . . . . . . . . . . . . . . . . 122 


\section{List of Tables}

3.1 Parameter Values in the Simulation . . . . . . . . . . . . . . . 48

4.1 Relaxation Times and Phenomenological Constants . . . . . . . . . . . . . . 90 


\section{Bibliography}

Adam, G. and M. Delbrück. 1968. Structural Chemistry and Biology, chapter Reduction of Dimensionality in Biological Processes, 198-215. W.H. Freeman and Company.

Almeida, P. F. and W. L. Vaz. 1995. Handbook of Biological Physics, chapter Lateral Diffusion in Membranes, 305-357. Elsevier Science B.V.

Almeida, P. F., W. L. Vaz, and T. Thompson. 2005. Lipid diffusion, free area and molecular dynamics simulations. Biophys. J. 88:4434-4438.

Almeida, P. F., W. L. Vaz, and T. E. Thompson. 1992. Lateral diffusion in the liquid phases of dimyristoylphosphatidylcholine/cholesterol lipid bilayers: A free volume analysis. Biochemistry 31:6739-6747.

Antonov, V., V. Petrov, A. Molnar, D. Predvoditelev, and A. Ivanov. 1980. The appearance of single-ion channels in unmodified lipid bilayer membranes at the phase transition temperature. Nature 283:585-586.

Antonov, V. F., A. A. Anosov, V. P. Norik, and E. Y. Smirnova. 2005. Soft perforation of planar bilayer lipid membranes of dipalmitoylphosphatidylcholine at the temperature of the phase transition from the liquid crystalline to the gel state. Eur. Biophys. J. 34:155-162.

Aragón, S. and R. Pecora. 1976. Fluorescence correlation spectroscopy as a probe of molecular dynamics. J. Chem. Phys. 64:1791-1803.

Arnold, A., M. Paris, and M. Auger. 2004. Anomalous diffusion in a gel-fluid lipid environment: A combined solid-state $\mathrm{nmr}$ and obstructed random-walk perspective. Biophys. J. $87: 2456-2469$.

Axelrod, D. 1983. Lateral motion of membrane proteins and biological function. J. Membr. Biol. $75: 1-10$.

Bagatolli, L. A. and E. Gratton. 1999. Two-photon fluorescence microscopy obeservation of shape changes at the phase transition in phospholipid giant unilamellar vesicles. Biophys. J. 77:2090-2101.

Bagatolli, L. A. and E. Gratton. 2000. Two photon fluorescence microscopy of coexisting lipid domains in giant unilamellar vesicles of binary phospholipid mixtures. BJ 78:290-305.

Bean, R. C. 1973. Protein-mediated mechanisms of variable ion conductance in thin lipid membranes. Membranes 2:409-477. 
Beattie, M. E., S. L. Veatch, B. L. Stottrup, and S. L. Keller. 2005. Sterol structure determines miscibility versus melting transitions in lipid vesicles. Biophys. J. 89:1760-1768.

Bienvenüe, A. and J. S. Marie. 1994. Modulation of protein function by lipids. Curr. Top. Membr. 40:319-354.

Biltonen, R. L. 1990. A statistical thermodynamic view of cooperative structural changes in phospholipid bilayer membranes: Their potential role in biological function. J. Chem. Thermodynamics 22:1-19.

Biltonen, R. L. and Q. Ye. 1993. Kinetics of the gel-to-liquid phase transition of binary lipid bilayers using volume perturbation calorimetry. Prog. Coll. Pol. Sci. 93:112-117.

Blume, A. 1983. Apparent molar heat capacities of phospholipids in aqueous dispersion. effects of chaing length and head group structure. Biochemistry 22:5436-5442.

Blume, A. and M. Hillmann. 1986. Dimyristoylphosphatidic acid/cholesterol bilayers. thermodynamic properties and kinetics of the phase transition as studied by the pressure jump relaxation technique. Eur. Biophys. J. 13:343-353.

Böckmann, R. A., A. Hac, T. Heimburg, and H. Grubmüller. 2003. Effect of sodium chloride on a lipid bilayer. Biophys. J. 858:1647-1655.

Bodin, S., H. Tronchère, and B. Payrastre. 2003. Lipid rafts are critical membrane domains in blood platelet activation processes. Biochim. Biophys. Acta 1610:247-257.

Boheim, G., W. Hanke, and H.-J. Eibl. 1980. Lipid phase transition in planar bilayer membrane and its effect on carrier- and pore-mediated ion transport. Proc. Natl. Acad. Sci. USA 77:3403-3407.

Bolen, E. J. and J. J. Sando. 1992. Effect of phospholipid unsaturation on protein kinase c activation. Biochemistry 31:5945-5951.

Bovill, J. G. 2000. Mechanisms of anaesthesia: Time to say farewell to the meyer-overton rule. Curr. Opin. Anaesth. 13:433-436.

Bretscher, M. 1972. Asymmetrical lipid bilayer structure for biological membranes. Nature New Biol. 236:11-12.

Brown, D. and E. London. 1998. Functions of lipid rafts in biological membranes. $A R C D B$ 14:111-136.

Brown, D. A. and E. London. 2000. Structure and function of sphingolipid- and cholesterolrich membrane rafts. J. Biol. Chem. 275:17221-17224.

Brown, M. F. 1994. Modulation of rhodopsin function by properties of the membrane bilayer. Chem. Phys. Lip. 73:159-180.

Burack, W. R. and R. L. Biltonen. 1994. Lipid bilayer heterogenities and modulation of phospholipase $a_{2}$ activity. Chem. Phys. Lip. 73:209-222. 
Caffrey, M. and G. W. Feigenson. 1981. Fluorescence quenching in model membranes. 3. relationship between calcium adenosinetriphosphatase enzyme activity and the affinity of the protein for phosphatidylcholines with different acyl chain characteristics. Biochemistry 20:1949-1961.

Cantor, R. S. 1997. The lateral pressure profile in membranes: A physical mechanism of general anesthesia. Biochemistry 36:2339-2344.

Cantor, R. S. 1999a. The influence of membrane lateral pressures on simple geometric models of protein conformational equilibria. Chem. Phys. Lip. 101:45-56.

Cantor, R. S. 1999b. Lipid composition and the lateral pressure profile in bilayers. Biophys. J. $76: 2625-2639$.

Cantor, R. S. 2001a. Bilayer partition coefficient of alkanols: Predicted effects of varying lipid composition. J. Phys. Chem. B 105:7550-7553.

Cantor, R. S. 2001b. Breaking the meyer-overton rule: Predicted effects of varying stiffness and interfacial activity on the intrinsic potency of anesthetics. Biophys. J. 80:2284-2297.

Caspary, D., M. Schröpfer, J. Lerchner, and G. Wolf. 1999. A high resolution ic-calorimeter for the determination of heats of absorption onto thin coatings. Therm. Acta 337:19-26.

Chapman, D. 1971. Liquid crystalline properties of phospholipids and biological membranes. Symp. Faraday Soc. 5:163-174.

Cheng, A., B. Hummel, A. Mencke, and M. Caffrey. 1994. Kinetics and mechanism of the barotropic lamellar gel/lamellar liquid crystal phase transition in fully hydrated dihexadecylphoshatidylethanolamine: A time-resolved x-ray diffraction study using pressure jump. Biophys. J. 67:293-303.

Clegg, R. M., E. L. Elson, and B. Maxfield. 1975. A new technique for optical observation of the kinetics of chemical reactions perturbed by small pressure changes. Biopolymers 14:883-887.

Clegg, R. M. and W. L. Vaz. 1985. Progress in Protein-Lipid Interactions, chapter 5. Translational Diffusion of Proteins and Lipids in Artifical Lipid Bilayer Membranes. A Comparison of Experiment with Theory, 173-229. Elsevier Science Publishers.

Corvera, E., O. G. Mouritsen, M. A. Singer, and M. J. Zuckermann. 1992. The permeability and the effect of acyl-chain length for phospholipid bilayers containing cholesterol: Theory and experiment. Biochim. Biophys. Acta 1107:261-270.

Crawford, M. S., B. Gerstein, A.-L. Kuo, and C. Wade. 1980. Diffusion in rigid bilayer membranes. use of combined multiple pulse and multiple pulse gradient techniques in nuclear magnetic resonance. J. Am. Chem. Soc. 102:3728-3732.

Cruzeiro-Hansson, L. and O. G. Mouritsen. 1988. Passive ion permeability of lipid membranes modelled via lipid-domain interfacial area. Biochim. Biophys. Acta 944:63-72.

Czeslik, C., J. Erbes, and R. Winter. 1997. Lateral organization of binary-lipid membranes- evidence for fractal-like behaviour in the gel-fluid coexistence region. Europhys. Lett. 37:577582 . 
de Almeida, R. F., L. M. Loura, A. Fedorov, and M. Prieto. 2002. Nonequilibrium phenomena in the phase separation of a two-component lipid bilayer. Biophys. J. 82:823-834.

Devaux, P. F. 1991. Static and dynamic lipid asymmetry in cell membranes. Biochemistry 30:1163-1173.

Dibble, A. R. G., A. K. Hinderliter, J. Sando, and R. L. Biltonen. 1996. Lipid lateral heterogeneity in phosphatidylcholine/phosphatidylserine/diacylglycerol vesicles and its influence on protein kinase c activation. Biophys. J. 71:1877-1890.

Doniach, S. 1978. Thermodynamic fluctuations in phospholipid bilayer. J. Chem. Phys. 68:4912-4916.

Duch, D. S. and S. R. Levinson. 1987. Spontaneous opening at zero membrane potential of sodium channels from eel electroplax reconstituted into lipid vesicles. J. Mol. Biol. 98:57-68.

Dumas, F., M. C. Lebrun, and J.-F. Tocanne. 1999. Is the protein/lipid hydrophobic matching principle relevant to membrane organization and function? FEBS Lett. 458:271-277.

Ebel, H., P. Grabitz, and T. Heimburg. 2001. Enthalpy and volume changes in lipid membranes. i. the proportionality of heat and volume changes in the lipid melting transition and its implication for the elastic constants. J. Phys. Chem. B 105:7353-7360.

Edidin, M. 1992. The variety of cell surface membrane domains. Com. Mol. Cell Biol. 8:73-82.

Edidin, M. 1996. Getting there is only half the fun. Curr. Top. Membr. 43:1-13.

Ehrenberg, M. and R. Rigler. 1974. Rotational brownian motion and fluorescence intensity fluctuations. Chem. Phys. 4:390-401.

Eigen, M. and R. Rigler. 1994. Sorting single molecules: Application to diagnostics and evolutionary biotechnology. Proc. Natl. Acad. Sci. USA 91:5740-5747.

Einstein, A. 1905. über die on der molekularkinetischen theorie der wärme geforderte bewegung von in ruhenden flüssigkeiten suspendierten teilchen. Ann. Phys. (Leipzig) 17:549-560.

Einstein, A. 1906. Zur theorie der brownschen bewegung. Ann. Phys. (Leipzig) 19:371-381.

Elamrani, K. and A. Blume. 1983. Phase transition kinetics of phosphatidic acid bilayers. a pressure jump relaxation study. Biochemistry 22:3305-3311.

Elson, E. and D. Magde. 1974. Fluorescence correlation spectroscopy i. conceptual basis and theory. Biopolymers 13:1-27.

Ermishkin, L. N., K. M. Kasumov, and V. M. Potzeluyev. 1976. Single ionice channels induced in lipid bilayers by polyene antibiotics amphotericin b and nystatine. Nature 262:698-699.

Falck, E., M. Patra, M. Karttunen, M. T. Hyvönen, and I. Vattulainen. 2004. Lessons of slicing membranes: Interplay of packing, free area, and lateral diffusion in phospholipid/cholesterol bilayers. Biophys. J. 87:1076-1091. 
Feder, T. J., I. Brust-Mascher, J. P. Slattery, B. Baird, and W. W. Webb. 1996. Constrained diffusio or immobile fraction on cell surfaces: A new interpretation. Biophys. J. 70:27672773.

Feller, S. E. 2000. Molecular dynamics simulations of lipid bilayers. Curr. Opin. Colloid Interface Sci. 5:217-223.

Fidorra, M. 2004. Untersuchung des Phasenverhaltens von Membranen durch konfokale Mikroskopie und Kalorimetrie. Master's thesis, University of Göttingen.

Franks, N. and W. Lieb. 1994. Molecular and cellular mechanisms of general anaesthesia. Nature 367:607-614.

Gabriel, N. E., N. V. Agman, and M. F. Roberts. 1987. Enzymatic hydrolysis of short-chain leithin/long-chain phospholipid unilamellar vesicles: Sensitivity of phospholipases to matrix phase state. Biochemistry 26:7409-7418.

Galla, H., W. Hartmann, U. Theilen, and E. Sackmann. 1979. On two-dimensional passive random walk in lipid bilayers and fluid pathways in biomembranes. J. Membr. Biol. 48:215236 .

Gennis, R. B. 1989. Biomembranes. Molecular Structure and Function. Springer.

Genz, A. and J. F. Holzwarth. 1986. Dynamic fluorescence measurements on the main phase transition of dipalmytoylphosphatidylcholine vesicles. Eur. Biophys. J. 13:323-330.

Genz, A., J. F. Holzwarth, and T. Tsong. 1986. The influence of cholesterol on the main phase transition of unilamellar dipalmytoylphosphatidylcholine vesicles. a differential scanning calorimetry and iodine laser t-jump study. Biophys. J. 50:1043-1051.

Glauber, R. 1963. Time-dependent statistics of the ising model. J. Math. Phys. 2:294-307.

Grabitz, P., V. P. Ivanova, and T. Heimburg. 2002. Relaxation kinetics of lipid membranes and its relation to the heat capacity. Biophys. J. 82:299-309.

Grainger, D., A. Reichert, H. Ringsdorf, and C. Salesse. 1989. An enzyme caught in action: Direct imaging of hydrolytic function and domain formation of phospholipase $a_{2}$ in phosphatidylcholine monolayers. FEBS Lett. 252:73-82.

Gruenewald, B. 1982. On the phase transition kinetics of phospholipid bilayers. relaxation experiments with detection of fluorescence anisotropy. Biochim. Biophys. Acta 687:71-78.

Gruenewald, B., A. Blume, and F. Watanabe. 1980. Kinetic investigations on the phase transition of phospholipid bilayers. Biochim. Biophys. Acta 597:41-52.

Gudmundsson, M. L. 2004. Relaxation time measurements on lipid-peptide systems. Bachelor's Thesis, University of Copenhagen.

Hac, A. E. 2004. Diffusion Processes in Membranes Containing Coexisting Domains Investigated by Fluorescence Correlation Spectroscopy. Ph.D. thesis, University of Göttingen. 
Hac, A. E., H. M. Seeger, M. Fidorra, and T. Heimburg. 2005. Diffusion in two-component lipid membranes- a fluorescence correlation spectroscopy and monte carlo simulation study. Biophys. J. 88:317-333.

Halstenberg, S., T. Heimburg, T. Hianik, U. Kaatze, and R. Krivanek. 1998. Cholesterolinduced variations in the volume and enthalpy fluctuations of lipid bilayers. Biophys. $J$. $75: 264-271$.

Halstenberg, S., W. Schrader, P. Das, J. Bhattacharjee, and U. Kaatze. 2003. Critical fluctuations in the domain structure of lipid membranes. J. Chem. Phys. 118:5683-5691.

Heerklotz, H. 2004. The microcalorimetry of lipid membranes. Journal of Physics: Condensed Matter 16:R441-R467.

Heimburg, T. 1998. Mechanical aspects of membrane thermodynamics. estimation of the mechanical properties of lipid membranes close to the chain melting transition from calorimetry. Biochim. Biophys. Acta 1415:147-162.

Heimburg, T. 2000. A model for the pretransition: Coupling of ripple formation with the chain-melting transition. Biophys. J. 78:1154-1165.

Heimburg, T. and R. L. Biltonen. 1996. A monte carlo simulation study of protein-induced heat capacity changes and lipid-induced protein clustering. Biophys. J. 70:84-96.

Heimburg, T. and A. D. Jackson. 2005. On solition propagation in biomembranes and nerves. Proc. Natl. Acad. Sci. USA 102:9790-9795.

Heimburg, T. and A. D. Jackson. 2006. General anesthetics. in preparation .

Helms, J. B. and C. Zurzolo. 2004. Lipids as targeting signals: Lipid rafts and intracellular trafficking. Traffic 5:247-254.

Hilgemann, D. W. 2003. Getting ready for the decade of the lipids. Ann. Rev. Physiol. 65:697-700.

Hill, T. 1962. Introduction to Statistical Thermodynamics, 2nd Edition. Addison-Wesley.

Hinderliter, A., R. L. Biltonen, and P. F. Almeida. 2004. Lipid modulation of proteininduced membrane domains as a mechanism for controlling signal transduction. Biochemistry 43:7102-7110.

Hinz, H.-J. and J. M. Sturtevant. 1972. Calorimetric studies of dilute aqueous suspensions of bilayers formed from synthetic l- $\alpha$-lecithins. J. Biol. Chem. 247:6071-6075.

Hladky, S. B. and D. A. Haydon. 1970. Discretness of conductance change in bimolecular lipid membranes in the prescence of certain antibiotics. Nature 225:451-453.

Hodgkin, A. and A. Huxley. 1952. A quantitative description of membrane current and its application to conduction and excitation in nerve. J. Physiol. (London) 117:500-544.

Holzwarth, J. F. 1989. The Enzyme Catalysis Process: Energetics, Mechanism and Dynamics, chapter Structure and Dynamics of Phospholipid Membranes From Nanoseconds to Seconds, 383-411. Plenum Publishing Group. 
Holzwarth, J. F. and F. Rys. 1984. Beobachtung einer kritischen trübung und verlangsamung am hauptphasenübergang von phospholipid-membranen, bestimmt mit der laser-temperatursprungmethode. Prog. Coll. Pol. Sci. 69:109-112.

Hønger, T., K. Jørgensen, R. L. Biltonen, and O. G. Mouritsen. 1996. Systematic relationship between phospholipase $a_{2}$ activity and dynamic lipid bilayer microheterogeneity. Biochemistry 35:9003-9006.

Hønger, T., K. Jørgensen, D. Stokes, R. L. Biltonen, and O. G. Mouritsen. 1997. Phospholipase $a_{2}$ activity and physical properties of lipid-bilayer substrates. Methods Enzymol. 286:168-190.

Hughes, B., B. Pailthorpe, and L. White. 1982. Extraction of membrane microviscosity from translational and rotational diffusion coefficients. Biophys. J. 37:673-676.

Ikonen, E. 2001. Roles of lipid rafts in membrane transport. Curr. Opin. Cell Biol. 13:470477 .

Ipsen, J. H., O. Karlström, O. G. Mouritsen, H. Winterström, and M. J. Zuckermann. 1987. Phase equilibria in the lecithin-cholesterol system. Biochim. Biophys. Acta 905:162-172.

Ivanova, V. P. 2000. Theoretical and Experimental Study of Protein-Lipid Interaction. Ph.D. thesis, University of Göttingen.

Ivanova, V. P., I. M. Makarov, T. E. Schäffer, and T. Heimburg. 2003. Analyzing heat capacity profiles of peptide-containing membranes: Cluster formation of gramicidin a. Biophys. J. 84:2427-2439.

Jackson, M. B. and J. M. Sturtevant. 1977. Studies of the lipid phase transitions of escherichia coli by high sensitivity differential scanning calorimeter. J. Biol. Chem. 252:4749-4751.

Jacobson, K., E. D. Sheets, and R. Simson. 1995. Revisiting the fluid mosaic model of membranes. Science 268:1441-1442.

Jain, M. K. and H. B. White. 1977. Long-range order in biomembranes. Adv. Lipid Res. $15: 1-60$.

Jain, M. K. and L. V. Wray. 1978. Partition coefficients of alkanols in lipid bilayer/water. Biochem. Pharm. 27:1294-1296.

Janiak, M., D. Small, and G. Shipley. 1979. Temperature and compositional dependence of the structure of hydrated dimyristoyl lecithin. J. Biol. Chem. 254:6068-6078.

Jensen, M. O. and O. G. Mouritsen. 2004. Lipids do influence protein function- the hydrophobic mismatch revisited. Biochim. Biophys. Acta 1666:205-226.

Jerala, R., P. F. Almeida, and R. L. Biltonen. 1996. Simulation of the gel-fluid transition in a membrane composed of lipids with two connected acyl chains: Application of a dimer-move step. Biophys. J. 71:609-615.

Johnson, M. L., T. C. Winter, and R. L. Biltonen. 1983. The measurement of the kinetics of lipid phase transitions: A volume perturbation kinetic calorimeter. Analyt. Biochem. 128:1-6. 
Johnston, D. and S. M.-S. Wu. 1997. Foundations of Cellular Neurophysiology. MIT Press.

Jørgensen, K., A. Klinger, and R. L. Biltonen. 2000. Nonequilibrium lipid domain growth in the gel-fluid two-phase region of a $d c_{16} p c-d c_{22} p c$ lipid mixture investigated by monte carlo simulation, ft-ir, and fluorescence spectroscopy. J. Phys. Chem. B 104:11763-11773.

Jørgensen, K. and O. G. Mouritsen. 1995. Phase separation dynamics and lateral organization of two-component lipid membranes. Biophys. J. 95:942-953.

Jørgensen, K., M. M. Sperotto, O. G. Mouritsen, J. H. Ipsen, and M. J. Zuckermann. 1993. Phase equilibria and local structure in binary lipid bilayers. Biochim. Biophys. Acta 1152:135-145.

Kaasgaard, T., O. G. Mouritsen, and K. Jørgensen. 2002. Lipid domain formation and ligandreceptor distribution in lipid bilayer membranes investigated by atomic force microscopy. FEBS Lett. 515:29-34.

Kahya, N., D. A. Wiersma, B. Poolman, and D. Hoekstra. 2002. Spatial organization of bacteriorhodopsin in model membranes. light induced mobility changes. J. Biol. Chem. 277:39304-39311.

Kamp, J. A. F. O. D., J. D. Gier, and L. V. Deenen. 1974. Hydrolysis of phosphatidylcholine liposomes by pancreatic phospholipase $a_{2}$ at the phase transition temperature. Biochim. Biophys. Acta 345:253-256.

Kamp, J. A. F. O. D., M. T. Kauerz, and L. V. Deenen. 1975. Action of pancreatic phopsholipase $a_{2}$ on phosphatidylcholine bilayers in different physical states. Biochim. Biophys. Acta 406:169-177.

Kappel, T., R. H. Anken, W. Hanke, and H. Rahmann. 2000. Gangliosides affect membranechannel activities dependent on ambient temperature. Cell. Mol. Neurobiol. 20:579-590.

Kaufmann, K., W. Hanke, and A. Corcia. 1989. Ion Channels. Ion Channel Fluctuation in Pure Lipid Bilayer Membranes: Control by Voltage. Caruaru.

Kaufmann, K. and I. Silman. 1980. The induction of ion channels through excitable membranes by acetylcholinesterase. Naturwissenschaften 67:608-610.

Kaufmann, K. and I. Silman. 1983a. The induction by protons of ion channels through lipid bilayer membranes. Biophys. Chem. 18:89-99.

Kaufmann, K. and I. Silman. 1983b. Proton-induced ion channels through lipid bilayer membranes. Naturwissenschaften 70:147-149.

Keynes, R. 1951. The ionice movements during nervous activity. J. Physiol. (London) 114:119-150.

Kharakoz, D. P. 2001. Phase-transition driven synaptic exocytosis: A hypothesis and its physiological and evolutionary implications. Bioscience Rep. 21:801-830.

Kikkawa, U., A. Kishimoto, and Y. Nishizuka. 1989. The protein kinase c family: Heterogeneity and its implications. Ann. Rev. Biochem. 58:31-44. 
Kobatake, Y., I. Tasaki, and A. Watanabe. 1971. Phase transition in membrane with reference to nerve excitation. Adv. Biophys. 2:1-31.

Kondepudi, D. and I. Prigogine. 1998. Modern Thermodynamics: From Heat Engines to Dissipative Structures. John Wiley \& Sons.

Korlach, J., P. Schwille, W. W. Webb, and G. Geigenson. 1999. Characterization of lipid bilayer phases by confocal microscopy and fluorescence correlation spectroscopy. Proc. Natl. Acad. Sci. USA 96:8461-8466.

Koynova, R. and M. Caffrey. 1998. Phases and phase transitions of the phosphocholines. Biochim. Biophys. Acta 1376:91-145.

Krasowski, M. and N. Harrison. 1999. General anesthetic action on ligand-gated ion channels. Cell. Mol. Life Sc. 55:1278-1303.

Kubo, R. 1966. The fluctuation-dissipation theorem. Rep. Prog. Phys. 29: Part I:255-284.

Kuo, A.-L. and C. G. Wade. 1979. Lipid lateral diffusion by pulsed nuclear magnetic resonance. Biochemistry 18:2300-2308.

L'Ecuyer, P. 1988. Efficient and portable combined random number generators. Com. of ACM 31:742-751.

Lee, A. G. 1976a. Interactions between anesthetics and lipid mixtures. normal alcohols. Biochemistry 15:2448-2454.

Lee, A. G. 1976b. Model for action of local anaesthetics. Nature 262:545-548.

Lee, A. G. 1977. Lipid phase transitions and phase diagrams ii. mixtures involving lipids. Biochim. Biophys. Acta 472:285-344.

Lee, A. G. 1991. Lipids and their effects on membrane proteins: Evidence against a role for fluidity. Prog. Lipid Res. 30:323-348.

Lee, A. G. 2003. Lipid-protein interactions in biological membranes: A structural perspective. Biochim. Biophys. Acta 1612:1-40.

Lee, A. G. 2004. How lipids affect the activities of integral membrane proteins. Biochim. Biophys. Acta 1666:62-87.

Lee, J. and J. Kosterlitz. 1991. Finite-size scaling and monte carlo simulations of first-order phase transitions. Phys. Rev. B 43:3265-3277.

Lerchner, J., R. Oehmgen, G. Wolf, P. L. Oarlouer, and J.-L. Daudon. 1998. Supermicrocalorimeteric devices for the investigation of small samples. High Temperature- High Pressures 30:701-708.

Lerchner, J., A. Wolf, and G. Wolf. 1999. Recent developments in integrated circuit calorimetry. J. Therm. Analy. Cal. 57:241-251.

Lev, A., Y. Korchev, T. Rostovtseva, C. Bashford, D. Edmonds, and C. Pasternak. 1993. Rapid switching of ion current in narrow pores: Implication for biological ion channels. Proc. Roy. Soc. Lond. B 252:187-192. 
Lichtenberg, D., G. Romero, M. Menashe, and R. L. Biltonen. 1986. Hydrolysis of dipalmytoylphosphatidylcholine large unilamellar vesicles by porcine pancreatic phospholipase $a_{2}$. J. Biol. Chem. 261:5334-5340.

Lin, L.-N., J. F. Brandts, J. M. Brandts, and V. Plotnikov. 2002. Determination of volumetric properties properties of proteins and other solutes using pressure perturbation calorimetry. Analyt. Biochem. 302:144-160.

Mabrey, S. and J. M. Sturtevant. 1976. Investigation of phase transitions of lipids and lipid mixtures by high sensitivity differential scanning calorimetry. Proc. Natl. Acad. Sci. USA 73:3862-3866.

Magde, D., E. Elson, and W. Webb. 1972. Thermodynamic fluctuations in a reacting systemmeasurement by fluorescence correlation spectroscopy. Phys. Rev. Lett. 29:705-708.

Magde, D., E. Elson, and W. W. Webb. 1974. Fluorescence correlation spectroscopy ii. an experimental realization. Biopolymers 13:29-61.

Makarov, I. 2005. Theoretical and Experimental Description of Permeability of PeptideContaining Membranes. Ph.D. thesis, University of Göttingen.

Marcelja, S. 1973. Molecular model for phase transition in biological membranes. Nature 241:451-453.

Marcelja, S. 1974. Chain ordering in liquid crystals ii. structure of bilayer membranes. Biochim. Biophys. Acta 367:165-176.

Marcelja, S. 1976. Lipid-mediated protein interaction in membranes. Biochim. Biophys. Acta 455:1-7.

Matteis, M. A. and A. Godi. 2004. Protein-lipid interactions in membrane trafficking at the golgi comlpex. Biochim. Biophys. Acta 1666:264-274.

McCloskey, M. and M.-M. Poo. 1984. Protein diffusion in cell membranes: Some biological implications. Int. Rev. Cytol. 87:19-81.

McElhaney, R. N. and K. A. Souza. 1976. The relationship between environmental temperature, cell growth and the fluidity and physical state of the membrane lipids in Bacillus Stearothermophilus. Biochim. Biophys. Acta 443:348-359.

Melchior, D. L. and J. M. Steim. 1976. Thermotropic transitions in biomembranes. Ann. Rev. Biophys. Bioeng. 5:205-238.

Melo, E. C., I. M. Lourtie, M. B. Sankram, and T. E. Thompson. 1992. Effects of domain connection and disconnection on the yields of in-plane bimolecular reactions in membranes. Biophys. J. 63:1506-1512.

Mengel, B. and M. Christiansen. 2005. Influence of anaesthetics on the melting transition of lipid membranes. Bachelor's Thesis, University of Copenhagen.

Metropolis, N., A. Rosenbluth, M. Rosenbluth, A. Teller, and E. Teller. 1953. Equation of state calculations by fast computing machines. J. Chem. Phys. 21:1087-1092. 
Meyer, H. 1899. Welche eigenschaft der anaesthetica bedingt ihre narkotische wirkung? Naunyn-Schmiedeberg's Arch. Exp. Pathol. Pharmakol. 42:109-118.

Michonova-Alexova, E. I. and I. P. Sugar. 2002. Component and state separation in dmpc/dspc lipid bilayers:a monte carlo simulation study. Biophys. J. 83:1820-1833.

Mitaku, S. and T. Date. 1982. Anomalies of nanosecond ultrasonic relaxation in the lipid bilayer transition. Biochim. Biophys. Acta 688:411-421.

Mouritsen, O. and M. Bloom. 1984. Mattress model of lipid-protein interactions in membranes. Biophys. J. 46:141-153.

Mouritsen, O. G. 1990. Molecular Description of Biological Membrane Components by Computer Aided Conformational Analysis, chapter Computer Simulation of Cooperative Phenomena in Lipid Membranes, 3-83. CRC Press.

Mouritsen, O. G. and K. Jørgensen. 1995. Micro-, nano- and meso-scale heterogeity of lipid bilayers and its influence on macroscopic membrane properties. Mol. Membr. Biol. 12:1520 .

Mouritsen, O. G. and M. J. Zuckermann. 2004. What's so special about cholesterol. Lipids 39:1101-1113.

Mukherjee, S. and F. R. Maxfield. 2000. Role of membrane organization and membrane domains in endocytic lipid trafficking. Traffic 1:203-211.

Nagano, H., H. Yao, and K. Ema. 1995. Dynamics heat capacity at the gel to liquid-crystalline phase transition in large unilamellar vesicles of dimyristoylphosphatidylcholine in the ultralow frequency region. Phys. Rev. E 51:3363-3367.

Nagle, J. F. 1973. Theory of biomembrane phase transitions. J. Chem. Phys. 58:252-264.

Neher, E. and B. Sakmann. 1976. Single-channel currents recorded from membrane of denervated frog muscle fibres. Nature 260:799-802.

Neher, E., B. Sakmann, and J. Steinbach. 1978. The extracellular patch clamp: A method for resolving currents through individual open channels in biological membranes. Pflügers Arch. Eur. J. Physiol. 375:219-228.

Nielsen, L. K., T. Bjørnholm, and O. G. Mouritsen. 2000a. Fluctuations caught in the act. Nature 404:352.

Nielsen, L. K., A. Vishnyakov, K. Jørgensen, T. Bjørnholm, and O. G. Mouritsen. 2000b. Nanometre-scale structure of fluid lipid membranes. J. Phys.: Condens. Matter 12:A309A314.

Nishizuka, Y. 1989. Studies and perspectives of protein kinase c. Science 233:305-312.

Oliynyk, V. 2005. Experimental Study of Lipid Membranes: Lipid Domain Formation and Peptide Aggregation. Ph.D. thesis, University of Göttingen. 
Orädd, G., G. Lindblom, and P. W. Westerman. 2002. Lateral diffusion of cholesterol and dimyristoylphosphatidylcholine in a lipid bilayer measured by pulsed field gradient $\mathrm{nmr}$ spectroscopy. Biophys. J. 83:2702-2704.

Overton, E. 1901. Studien über die Narkose zugleich ein Beitrag zur allgemeinen Pharmakologie. Fischer.

Papahadjopoulos, D., K. Jacobsen, S. Nir, and T. Isac. 1973. Phase transition in phospholipid vesicles. fluoresence polarization and permeability measurements conerning the effect of temperature and cholesterol. Biochim. Biophys. Acta 311:330-348.

Parsegian, V. A. 1995. The cows or the fence? Mol. Membr. Biol. 12:5-7.

Paula, S. and D. Deamer. 1999. Membrane permeability barriers to ionic and polar solutes. Curr. Top. Membr. 48:77-95.

Peet, M., I. Glen, and D. F. Horrobin, editors. 1999. Phospholipid Spectrum Disorder in Psychiatry. Marius Press.

Pfeiffer, W., G. Schlossbauer, W. Knoll, B. Farago, A. Steyer, and E. Sackmann. 1988. Ultracold neutron scattering study of local lipid mobility in bilayer membranes. J. Phys. France 49:1077-1082.

Pink, D. A. and D. Chapman. 1979. Protein-lipid interactions in bilayer membranes: A lattice model. Proc. Natl. Acad. Sci. USA 76:1542-1546.

Pink, D. A., T. J. Green, and D. Chapman. 1980. Raman scattering in bilayers of saturated phosphatidylcholines. experiment and theory. Biochemistry 19:349-356.

Plotnikov, V. V., J. M. Brandts, L.-N. Lin, and J. F. Brandts. 1997. A new ultrasensitive scanning calorimeter. Analyt. Biochem. 250:237-244.

Pollack, G. H. 2001. Cells, Gels and the Engines of Life. Ebner and Sons.

Pollakowski, D. 2003. Thermodynamische und Strukturelle Untersuchungen an künstlichen und biologischen Membranen. Grundlegende Eigenschaften und der Einfluss kleiner Moleküle. Master's thesis, University of Göttingen.

Polson, J., I. Vattulainen, H. Zhu, and M. J. Zuckermann. 2001. Simulation study of lateral diffusion in lipid-sterol bilayer mixtures. Eur. Phys. J. E 5:485-497.

Press, W., S. Teukolsky, W. Vetterling, and B. Flannery. 1997. Online Version Numerical Recipes in C, Second Edition, Reprint 1997, Cambridge University Press, chapter Random Numbers, 274-283.

Privalov, P., Y. V. Griko, and S. Y. Venyaminov. 1986. Cold denaturation of myoglobin. J. Mol. Biol. 190:487-498.

Reinert, J. C. and J. M. Steim. 1970. Calorimetric detection of a membrane-lipid phase transition in living cells. Science 168:1580-1582.

Rigler, R. and U. Mets. 1992. Diffusion of single molecules through a gaussian laser beam. SPIE 1921:239-248. 
Rigler, R., U. Mets, J. Widengren, and P. Kask. 1993. Fluorescence correlation spectrsocopy with high count rate and low background: Analysis of translational diffusion. Eur. Biophys. J. $22: 169-175$.

Ritchie, K., X.-Y. Shan, J. Kondo, K. Iwasawa, T. Fujiwara, and A. Kusumi. 2005. Detection of non-brownian diffusion in the cell membrane in single molecule tracking. Biophys. $J$. $88: 2266-2277$.

Sachs, F. and F. Qin. 1993. Gated, ion-selective channels observed with patch pipettes in the absence of membranes: Novel properties of gigaseal. Biophys. J. 65:1101-1107.

Sackmann, E. 1984. Biological Membranes, chapter Physical Basis of Trigger Processes and Membrane Structure, 105-143. Academic Press.

Saffman, P. and M. Delbrück. 1975. Brownian motion in biological membranes. Proc. Natl. Acad. Sci. USA 72:3111-3113.

Salinas, D. G., M. D. L. Feunte, and J. G. Reyes. 2005. Changes of enzyme activity in lipid signaling phathways related to substrate reordering. Biophys. J. 89:885-894.

Sandermann, H. 1978. Regulation of membrane enzymes by lipids. Biochim. Biophys. Acta 515:209-237.

Saxton, M. J. 1987. Lateral diffusion in an archipelago. the effect of mobile obstacles. Biophys. J. 52:989-997.

Saxton, M. J. 1990. Lateral diffusion in a mixture of mobile and immobile particles. a monte carlo study. Biophys. J. 58:1303-1306.

Saxton, M. J. 1992. Lateral diffusion and aggregation. Biophys. J. 61:119-128.

Saxton, M. J. 1994. Anomalous diffusion due to obstacles: A monte carlo study. Biophys. J. $66: 394-401$

Saxton, M. J. 1996. Anomalous diffusion due to binding: A monte carlo study. Biophys. J. 70:1250-1262.

Saxton, M. J. 1999. Lateral diffusion of lipids and proteins. Curr. Top. Membr. 48:229-282.

Scheidt, H. A., D. Huster, and K. Gawrisch. 2005. Diffusion of cholesterol and its precursors in lipid membranes studied by ${ }^{1} \mathrm{~h}$ pulsed field gradient magic angle spinning nmr. Biophys. J. 89:2504-2512.

Schlötzer, T. 2002. Domänen in Lipidmembranen: Untersuchungen zum Phasenverhalten von binären Lipidmischungen mit Kalorimetrie und konfokaler Fluoreszenzmikroskopie. Master's thesis, University of Göttingen.

Schneider, M. B., W. K. Chan, and W. W. Webb. 1983. Fast diffusion along defects and corrugations in phospholipid $p_{\beta}$ liquid crystals. Biophys. J. 43:157-165.

Schütz, G., H. Schindler, and T. Schmidt. 1997. Single-molecule microscopy on model membranes reveals anomalous diffusion. Biophys. J. 73:1073-1080. 
Schwille, P., J. Korlach, and W. W. Webb. 1999. Fluorescence correlation spectroscopy with single-molecule sensitivity on cell and model membranes. Cytometry 36:176-182.

Schwille, P., F.-J. Meyer-Almes, and R. Rigler. 1997. Dual-color fluorescence cross-correlation spectroscopy for mulicomponent diffusional analysis in solution. Biophys. J. 72:1878-1886.

Seeger, H. 2002. Theoretische und Experimentelle Beschreibung von Diffusionsprozessen in einem Zweikomponentigen Lipidsystem. Master's thesis, University of Göttingen.

Seeger, H. M., M. Fidorra, and T. Heimburg. 2005. Domain size and fluctuations at domain interfaces in lipid mixtures. Macro. Symposia 219:85-96.

Shin, Y.-K., U. Ewert, D. Budil, and J. Freed. 1991. Microscopic versus macroscopic diffusion in model membranes by electron spin resonance spectral-spatial imaging. Biophys. J. 59:950-957.

Simons, K. and E. Ikonen. 1997. Functional rafts in cell membranes. Nature 387:569-572.

Simons, K. and W. L. Vaz. 2004. Model systems, lipid rafts, and cell membranes. ARBMS 33:269-295.

Simson, R., E. D. Sheets, and K. Jacobson. 1995. Detection of temporary lateral confinement of membrane proteins using single-particle tracking analysis. Biophys. J. 69:989-993.

Singer, S. and G. L. Nicolson. 1972. The fluid mosaic model of the structure of cell membranes. Science 175:720-731.

Sonnleitner, A., G. Schütz, and T. Schmidt. 1999. Free brownian motion of individual lipid molecules in biomembranes. Biophys. J. 77:2638-2642.

Sperotto, M. M. and O. G. Mouritsen. 1991. Monte carlo simulation studies of lipid order parameter profiles near integral membrane proteins. Biophys. J. 59:261-269.

Spyropoulos, C. S. 1957. The effects of hydrostatic pressure upon the normal and narcotized nerve fiber. J. Gen. Physiol. 40:849-857.

Steim, J. M., M. E. Tourtellotte, K. C. Reinert, R. N. McElhaney, and R. L. Rader. 1969. Calorimetric evidence for the liquid-crystalline state of lipids in a biomembrane. Proc. Natl. Acad. Sci. USA 63:104.

Strehlow, U. and F. Jähnig. 1981. Electrostatic interactions at charged lipid membranes. kinetics of the electrostatically triggered phase transition. Biochim. Biophys. Acta 641:301310.

Sugar, I. and R. L. Biltonen. 2005. Lateral diffusion of molecules in two-component lipid bilayer: A monte carlo simulation study. J. Phys. Chem. B 109:7373-7386.

Sugar, I. P., R. L. Biltonen, and N. Mitchard. 1994. Monte carlo simulation of membranes: Phase transition of small unilamellar dipalmitoylphosphatidylcholine vesicles. Methods Enzymol. 240:569-593. 
Sugar, I. P., E. Michonova-Alexova, and P. L.-G. Chong. 2001. Geometrical properties of gel and fluid clusters in dmpc/dspc bilayers: Monte carlo simulation approach using a two-state model. Biophys. J. 81:2425-2441.

Sugar, I. P., T. E. Thompson, and R. L. Biltonen. 1999. Monte carlo simulation of twocomponent bilayers: Dmpc/dspc mixtures. Biophys. J. 76:2099-2110.

Tabony, J. and B. Perly. 1990. Quasielastic neutron scattering measurements of fast local translational diffusion of lipid molecules in phospholipid bilayers. Biochim. Biophys. Acta 1063:67-72.

Tamura, K., Y. Kaminoh, H. Kamaya, and I. Ueda. 1991. High pressure antagonism of alcohol effects on the main phase-transition temperature of phospholipid membranes: Biphasic response. Biochim. Biophys. Acta 1066:219-224.

Tasaki, I. and P. Byrne. 1992. Rapid structural changes in nerve fibers evoked by electrical current pulses. Biochem. Biophys. Research Comm. 188:559-564.

Tasaki, I. and P. Byrne. 1993. Rapid heat production associated with excitation of electric organs of the electric eel. Biochem. Biophys. Research Comm. 197:910-915.

Tasaki, I., A. Watanabe, R. Sandlin, and L. Carnay. 1968. Changes in fluorescence, turbidity, and birefringence associated with nerve excitation. Proc. Natl. Acad. Sci. USA 61:883-888.

Thompson, T., M. Sankram, R. Biltonen, D. Marsh, and W. Vaz. 1995. Effects of domain structure on in-plane reactions and interactions. Mol. Membr. Biol. 12:157-162.

Tokumasu, F., A. J. Jin, G. W. Feigenson, and J. A. Dvorak. 2003. Nanoscopic lipid domain dynamics revealed by atomic force microscopy. Biophys. J. 84:2609-2618.

Tomlin, S. L., A. Jenkins, L. W. R., and N. P. Franks. 1998. Stereoselective effects of etomidate optical isomers on gamma-aminobutyric acid type a receptors and animals. Anesthesiology 88:708-717.

Trandum, C., P. Westh, K. Jørgensen, and O. G. Mouritsen. 1999. Use of isothermal titration calorimetry to study the interaction of short-chain alcohols with lipid membranes. Therm. Acta 328:129-135.

Träuble, H. 1971. Phasenumwandlungen in lipiden. mögliche schaltprozesse in biologischen membranen. Naturwissenschaften 58:277-284.

Träuble, H. and H.-J. Eibl. 1974. Electrostatic effects on lipid phase transitions: Membrane structure and ionic environment. Proc. Natl. Acad. Sci. USA 71:214-219.

Trudell, J. R. 1977. A unitary theory of anesthesia based on lateral phase separations in nerve membranes. Anesthesiology 46:5-10.

Tsong, T. Y. 1974. Kinetics of the crystalline-liquid crystalline phase transition of dimyristoyl l- $\alpha$-lecithin bilayers. Proc. Natl. Acad. Sci. USA 1974:2684-2688.

Tsong, T. Y. and M. I. Kanehisa. 1977. Relaxation phenomena in aqueous dispersions of synthetic lecithins. Biochemistry 16:2674-2680. 
Ueda, I. and T. Yoshida. 1999. Hydration of lipid membranes and the action mechanisms of anesthetics and alcohols. Chem. Phys. Lip. 101:65-79.

van den Bosh, H. 1980. Intracellular phospholipase a. Biochim. Biophys. Acta 604:191-246.

van Osdol, W. W., R. L. Biltonen, and M. Johnson. 1989. Measuring the kinetics of membrane phase transitions. J. Biochem. Biopyhs. Methods 20:1-46.

van Osdol, W. W., M. L. Johnson, Q. Ye, and R. L. Biltonen. 1991. Relaxation dynamics of the gel to liquid crystalline transition of phosphatidylcholine bilayers. effects of chainlength and vesicle size. Biophys. J. 59:775-785.

van Osdol, W. W., Q. Yes, M. L. Johnson, and R. L. Biltonen. 1992. Effects of the anesthetic dibucaine on the kinetics of the gel-liquid crystalline transition of dipalmitoylphosphatidylcholine multilamellar vesicles. Biophys. J. 63:1011-1017.

Vaz, W. L. and P. F. Almeida. 1991. Microscopic versus macroscopic diffusion in onecomponent fluid phase lipid bilayer membranes. Biophys. J. 60:1553-1554.

Vaz, W. L. and P. F. F. Almeida. 1993. Phase topology and percolation in multi-phase lipid bilayers: Is the biological membrane a domain mosaic? Curr. Opin. in Struct. Biol. $3: 482-488$

Vaz, W. L., R. M. Clegg, and D. Hallmann. 1985. Translational diffusion of lipids in liquid crystalline phase phosphotidylcholine multibilayers. a comparison of experiment and theory. Biochemistry 24:781-786.

Vaz, W. L., M. Criado, V. M. Madeira, G. Schöllmann, and T. M. Jovin. 1982. Size dependence of the translational diffusion of large integral membrane proteins in liquid-crystalline phase lipid bilayers. a study using fluorescence recovery after photobleaching. Biochemistry 21:5608-5612.

Vaz, W. L., E. C. Melo, and T. E. Thompson. 1989. Translational diffusion and fluid domain connectivity in a two-component, two-phase phospholipid bilayer. Biophys. J. 56:869-876.

Wachsmuth, M., W. Wladeck, and J. Langowski. 2000. Aomalous diffusion of fluorescent probes inside living cell nuclei investigated by spatially-resolved fluorescence correlation spectroscopy. J. Mol. Biol. 298:677-689.

Wiggins, P. and R. Phillips. 2005. Membrane-protein interactions in mechanosensitive channels. Biophys. J. 88:880-902.

Winter, R., A. Gabke, C.Czeslik, and P. Pfeifer. 1999. Power-law fluctuations in phaseseparated lipid membranes. Phys. Rev. E 60:7354-7359.

Woodbury, D. J. 1989. Pure lipid vesicles can induce channel-like conductances in planar bilayers. J. Mol. Biol. 109:145-150.

Yager, P. and W. L. Peticolas. 1982. The kinetics of the main phase transition of aqueous dispersions of phospholipids induced by pressure jump and monitored by raman spectroscopy. Biochim. Biophys. Acta 688:775-785. 
Yao, H., H. Nagano, Y. Kawase, and K. Ema. 1994. Slow relaxation process in the main phase transition of phosphatidylcholines studied with heat capacity spectroscopy. i. multilamellar vesicles. Biochim. Biophys. Acta 1212:73-79. 